
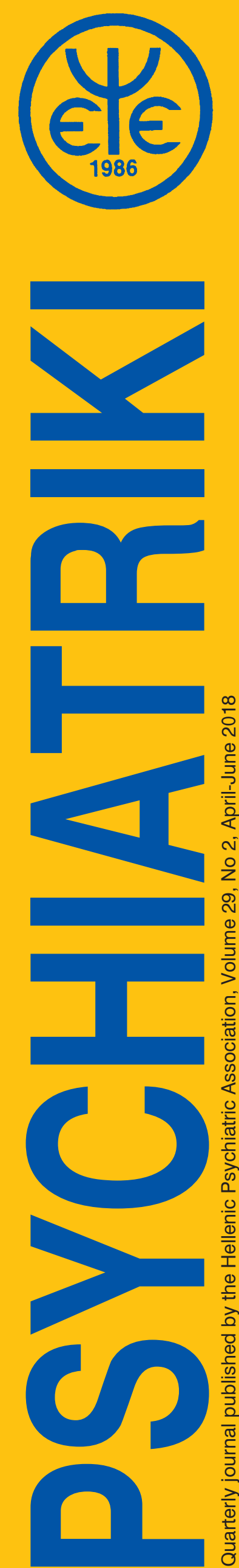

UYXIATPIKH

UYXIATPIKH

UYXIATPIKH

UYXIATPIKH

UYXIATPIKH

UYXIATPIKH

UYXIATPIKH

UYXIATPIKH

UYXIATPIKH

UYXIATPIKH

UYXIATPIKH

UYXIATPIKH

UYXIATPIKH

UYXIATPIKH

UYXIATPIKH

UYXIATPIKH

UYXIATPIKH

UYXIATPIKH

UYXIATPIKH

UYXIATPIKH

UYXIATPIKH

UYXIATPIKH

UYXIATPIKH

UYXIATPIKH

UYXIATPIKH

UYXIATPIKH

UYXIATPIKH

UYXIATPIKH

UYXIATPIKH

UYXIATPIKH

UYXIATPIKH

UYXIATPIKH

UYXIATPIKH

UYXIATPIKH

UYXIATPIKH

UYXIATPIKH

UYXIATPIKH

UYXIATPIKH

UYXIATPIKH

UYXIATPIKH

UYXIATPIKH

WYXIATPIKH

UYXIATPIKH

WYXIATPIKH

UYXIATPIKH

UYXIATPIKH

WYXIATPIKH

UYXIATPIKH

UYXIATPIKH

UYXIATPIKH

UYXIATPIKH

UYXIATPIKH

WYXIATPIKH

UYXIATPIKH

UYXIATPIKH

WYXIATPIKH

WYXIATPIKH

GR ISSN 1105-2333

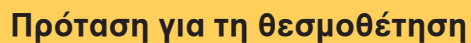

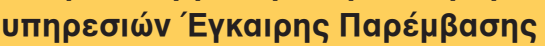

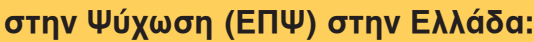

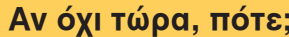

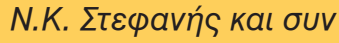

$$
\bullet \bullet \bullet
$$

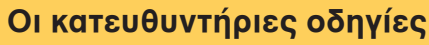

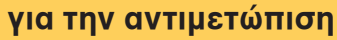

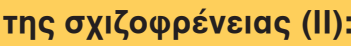

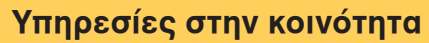

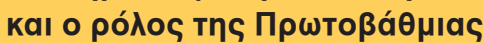
Фpovtídas Yyeías

M. Маруарітп каı бuv

$$
\bullet \bullet \bullet
$$

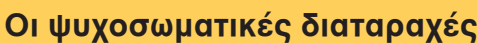

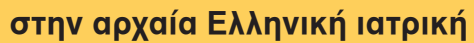
K. ^áıos kaı ouv

$$
\bullet \bullet \bullet
$$

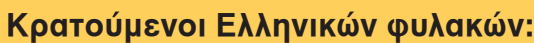

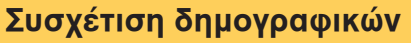

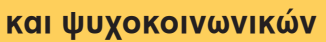

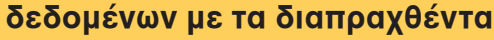

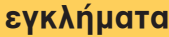

А. Апобтоло́поилос каı бuv

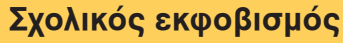

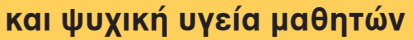

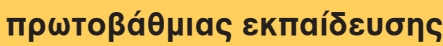

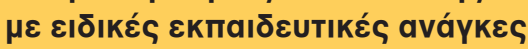

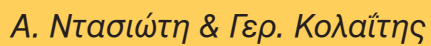

$\bullet \bullet \bullet$

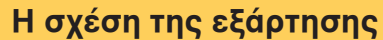

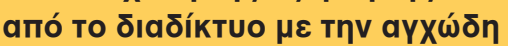

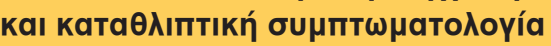

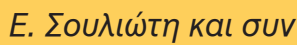

$$
\circ \circ
$$

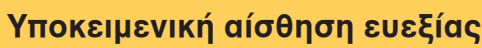

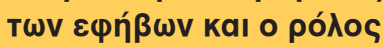

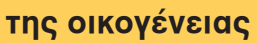

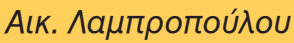

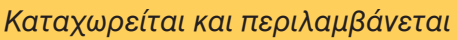
ota MEDLINE/PubMed,

Index Copernicus, Google Scholar EMBASE/Excerpta Medica, GFMER, CIRRIE, SCIRUS for Scientific Inf., EBSCOhost ${ }^{T M}$, PsychINFO kaı бто latrotek

(Scimago Journal Rank 2016 $=0.203$ )
WYXIATPIKH

WYXIATPIKH

WYXIATPIKH

UYXIATPIKH

WYXIATPIKH

UYXIATPIKH

WYXIATPIKH

WYXIATPIKH

WYXIATPIKH

WYXIATPIKH

WYXIATPIKH

WYXIATPIKH

WYXIATPIKH

WYXIATPIKH

UYXIATPIKH

WYXIATPIKH

UYXIATPIKH

UYXIATPIKH

UYXIATPIKH

UYXIATPIKH

UYXIATPIKH

UYXIATPIKH

UYXIATPIKH

WYXIATPIKH

UYXIATPIKH

UYXIATPIKH

UYXIATPIKH

UYXIATPIKH

UYXIATPIKH

UYXIATPIKH

WYXIATPIKH

UYXIATPIKH

UYXIATPIKH

UYXIATPIKH

UYXIATPIKH

UYXIATPIKH

WYXIATPIKH

WYXIATPIKH

W YXIATPIKH

WYXIATPIKH

UYXIATPIKH

WYXIATPIKH

WYXIATPIKH

UYXIATPIKH

WYXIATPIKH

UYXIATPIKH

UYXIATPIKH

UYXIATPIKH

UYXIATPIKH

UYXIATPIKH

UYXIATPIKH

WYXIATPIKH

WYXIATPIKH

WYXIATPIKH

WYXIATPIKH

WYXIATPIKH

ЧYXIATPIKH
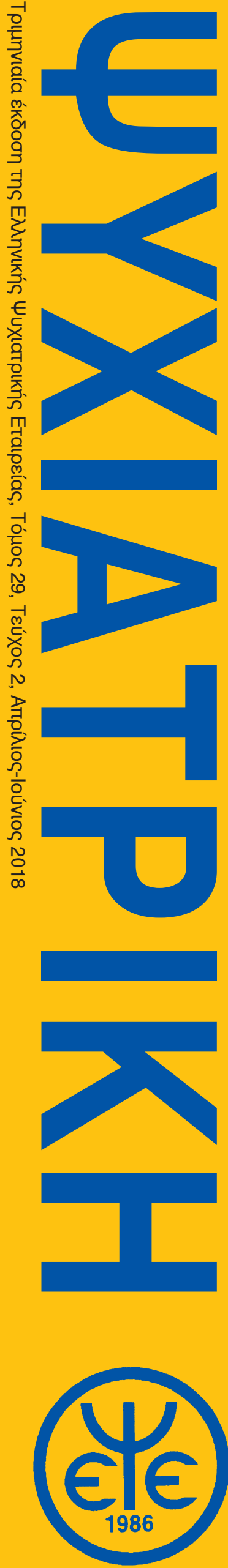


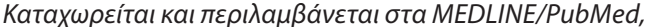
Index Copernicus, Google Scholar, EMBASE/Excerpta Medica, GFMER, CIRRIE, SCIRUS for Scientific Inf., EBSCOhost ${ }^{T M}$, PsychINFO кal oto latrotek

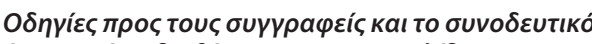

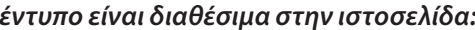
http://www.psychiatriki-journal.gr

\section{UYXIATPIKH}

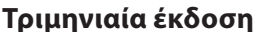

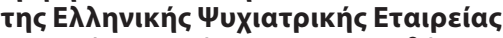

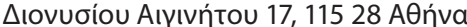

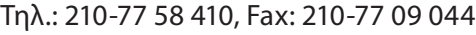

Екठо́тnc:

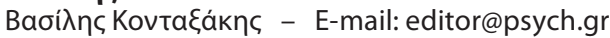

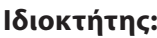

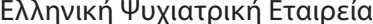

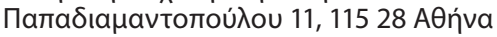

$\operatorname{T\eta \lambda } .: 210-7214184$

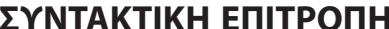

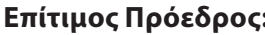

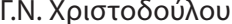

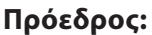

B. Kovтałáknc

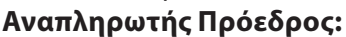

Г. Кшvбтаvтако́тои

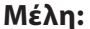

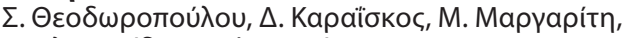

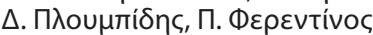

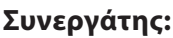

I. Zźpßaৎ

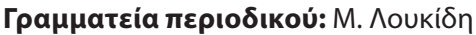

Indexed and included in MEDLINE/PubMed, Index Copernicus, Google Scholar, EMBASE/Excerpta Medica, GFMER, CIRRIE, SCIRUS for Scientific Inf., EBSCOhost ${ }^{T M}$

PsychINFO and in latrotek

Instructions to contributors and the submission form are available at the webpage

http://www.psychiatriki-journal.gr

\section{PSYCHIATRIKI}

Quarterly journal published

by the Hellenic Psychiatric Association

17, Dionisiou Eginitou str., 11528 Athens

Tel.: +30-210-77 58 410, Fax: +30-210-77 09044

Publisher:

Vassilis Kontaxakis - E-mail: editor@psych.gr

Owner:

Hellenic Psychiatric Association

11, Papadiamantopoulou str., 11528 Athens

Tel.: +30-210-72 14184

\section{EDITORIAL BOARD}

\section{Emeritus Editor:}

G.N. Christodoulou

Editor -in- Chief:

V. Kontaxakis

Associate Editor:

G. Konstantakopoulos

Members:

S. Theodoropoulou, D. Karaiskos, M. Margariti,

D. Ploumpidis, P. Ferentinos

Collaborator:

J. Zervas

Journal's secretariat: M. Loukidi
M. Abou-Saleh (UK)
H. Akiskal (USA)
G. Alexopoulos (USA)
N. Andreasen (USA)
S. Bloch (Australia)
M. Botbol (France)
N. Bouras (UK)
C. Höschl (Czech Rep.)
${ }^{\dagger} \mathrm{H}$. Ghodse (UK)
P. Gökalp (Turkey)
G. Ikkos (UK)
R.A. Kallivayalil (India)
M. Kastrup (Denmark)
K. Kirby (Australia)
V. Krasnov (Russia)

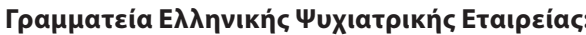

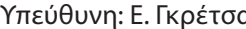

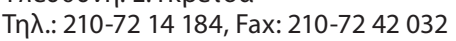

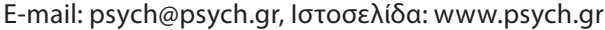

FB: E^^HNIKH $\Psi$ YXIATPIKH ETAIPEIA

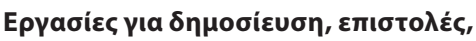

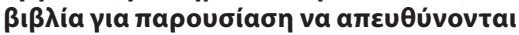

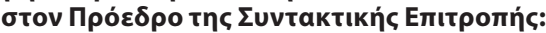

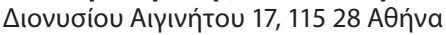

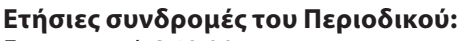

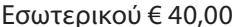

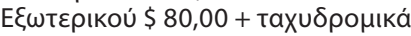

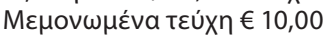

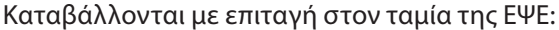

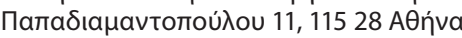

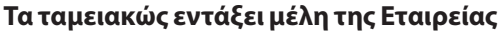

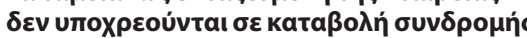

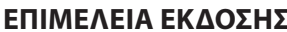 EN ISO 9001:2000}

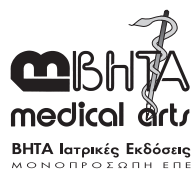

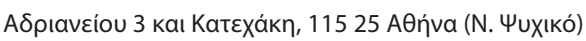
Tn入.: 210-67 14371 - 210-67 14 340, Fax: 210-67 15015 e-mail: betamedarts@otenet.gr

e-shop: www.betamedarts.gr

EN ISO 9001:2000

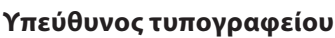

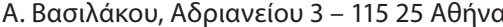

$\operatorname{T\eta } \lambda .210-6714340$

\section{VISORY BOARD}

D. Lecic-Tosevski (Serbia) G. Papakostas (USA)

C. Lyketsos (USA) G. Petrides (USA)

M. Maj (Italy)

A. Marneros (Germany)

J. Mezzich (USA)

R. Salokangas (Finland)

H.J. Möller (Germany)

R. Montenegro (Argentina) N. Tataru (Romania)

C. Pantelis (Australia) P. Tyrer (UK)

Secretariat of Hellenic Psychiatric Association: Head: H. Gretsa

Tel.: (+30) 210-72 14 184, Fax: (+30) 210-72 42032

E-mail: psych@psych.gr, Web-site:www.psych.gr

Manuscripts, letters, books for review should be addressed to the Editor: 17 Dionisiou Eginitou str., GR-115 28 Athens, Greece

Annual subscriptions of the Journal: $€ 40.00$ or $\$ 80.00$ + postage - each separate issue $€ 10.00$ are payable by check to the treasurer of the Hellenic Psychiatric Association: 11, Papadiamantopoulou str., GR-115 28 Athens

For the members of the Association in good standing subscription is free

EDITING EN ISO 9001:2000

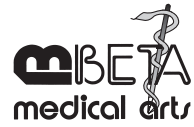

3, Adrianiou str., GR-115 25 Athens-Greece Tel.: (+30) 210-67 14371 - (+30) 210-67 14 340, Fax: (+30) 210-67 15015

e-mail: betamedarts@otenet.gr, e-shop:www.betamedarts.gr EN ISO 9001:2000

Printing supervision

A. Vassilakou, 3 Adrianiou str. - GR-115 25 Athens

Tel. (+30)-210-67 14340 
EAMHNIKH $\Psi$ YXIATPIKH ETAIPEIA

\section{DIOIKHTIKO EYMBOYAIO}

\begin{tabular}{|c|c|}
\hline 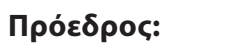 & 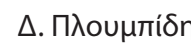 \\
\hline 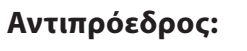 & 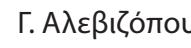 \\
\hline 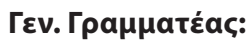 & 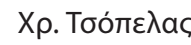 \\
\hline Tauiac: & ^. Марка́кп \\
\hline$\Sigma u ́ \mu \beta o u \lambda \circ$ เ: & 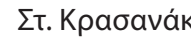 \\
\hline & В.П. Млс \\
\hline & X. Touגo \\
\hline ПЕІОАРХІКО $\Sigma Y$ & rMBOYAIO \\
\hline 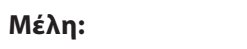 & B. $A \lambda \varepsilon \beta i \zeta о \varsigma$ \\
\hline & І. Гкıоuไદ́паৎ \\
\hline & 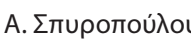 \\
\hline 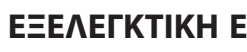 & ПІтРОПн \\
\hline 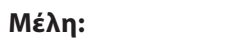 & B. Kovтałáknc \\
\hline & E. ¿ıoútn \\
\hline & N. Tלaßápac \\
\hline
\end{tabular}

\section{EחITIMOI ПPOEDPOI}

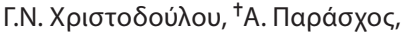

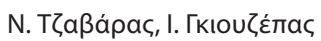

\section{EחITIMA MEAH}

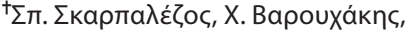

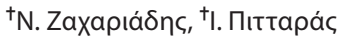

\begin{tabular}{|c|c|}
\hline \multicolumn{2}{|c|}{$\begin{array}{l}\text { ПЕРІФЕРЕІАКА ТМНMАТА } \\
\text { АӨНNQN }\end{array}$} \\
\hline 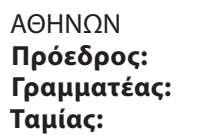 & 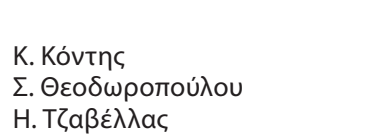 \\
\hline 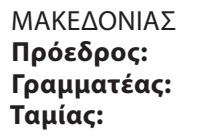 & 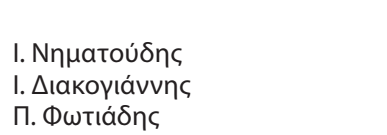 \\
\hline 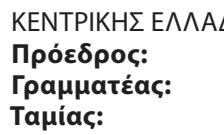 & 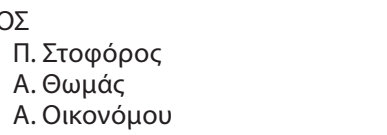 \\
\hline 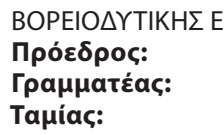 & 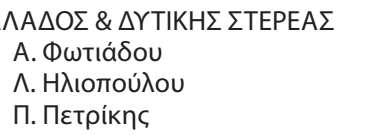 \\
\hline 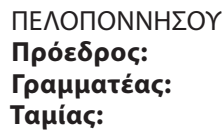 & 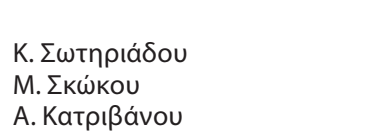 \\
\hline 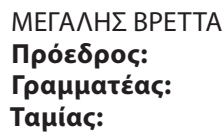 & 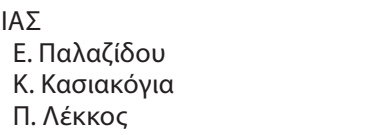 \\
\hline 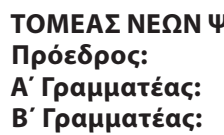 & 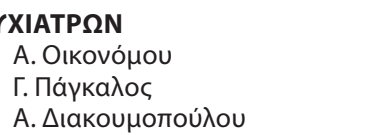 \\
\hline 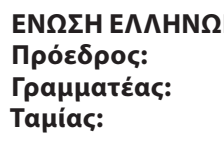 & 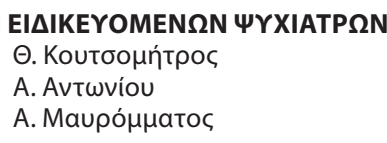 \\
\hline
\end{tabular}

HELLENIC PSYCHIATRIC ASSOCIATION

EXECUTIVE COUNCIL

$\begin{array}{ll}\text { Chairman: } & \text { D. Ploumpidis } \\ \text { Vice-Chairman: } & \text { G. Alevizopoulos } \\ \text { Secretary General: } & \text { Ch. Tsopelas } \\ \begin{array}{ll}\text { Treasurer: } & \text { L. Markaki } \\ \text { Consultants: } & \text { St. Krasanakis } \\ & \text { V.P. Bozikas } \\ & \text { Ch. Touloumis } \\ \text { DISCIPLINARY COUNCIL } & \text { V. Alevizos } \\ \text { Members: } & \text { J. Giouzepas } \\ & \text { A. Spyropoulou }\end{array}\end{array}$

FINANCIAL CONTROL COMMITTEE

$\begin{array}{ll}\text { Members: } & \text { V. Kontaxakis } \\ & \text { Ir. Siouti } \\ & \text { N. Tzavaras }\end{array}$

HONORARY PRESIDENTS

G.N. Christodoulou, ${ }^{\dagger}$ A. Paraschos, N. Tzavaras, J. Giouzepas

HONORARY MEMBERS

${ }^{\dagger} \mathrm{S}$. Scarpalezos, Ch. Varouchakis,

${ }^{\dagger} \mathrm{N}$. Zachariadis, ${ }^{\dagger}$ I. Pittaras

DIVISIONS

ATHENS

\begin{tabular}{|c|c|}
\hline $\begin{array}{l}\text { Chairman: } \\
\text { Secretary: } \\
\text { Treasurer: }\end{array}$ & $\begin{array}{l}\text { C. Kontis } \\
\text { S. Theodorop } \\
\text { E. Tzavellas }\end{array}$ \\
\hline $\begin{array}{l}\text { MACEDONIA } \\
\text { Chairman: } \\
\text { Secretary: } \\
\text { Treasurer: }\end{array}$ & $\begin{array}{l}\text { J. Nimatoudis } \\
\text { J. Diakoyiann } \\
\text { P. Fotiadis }\end{array}$ \\
\hline $\begin{array}{l}\text { CENTRAL GREECE } \\
\text { Chairman: } \\
\text { Secretary: } \\
\text { Treasurer: }\end{array}$ & $\begin{array}{l}\text { P. Stoforos } \\
\text { A. Thomas } \\
\text { A. Oikonomo }\end{array}$ \\
\hline $\begin{array}{l}\text { NORTHWESTERN } \\
\text { Chairman: } \\
\text { Secretary: } \\
\text { Treasurer: }\end{array}$ & $\begin{array}{l}\text { REECE } \\
\text { A. Fotiadou } \\
\text { L. Iliopoulou } \\
\text { P. Petrikis }\end{array}$ \\
\hline $\begin{array}{l}\text { PELOPONNESE } \\
\text { Chairman: } \\
\text { Secretary: } \\
\text { Treasurer: }\end{array}$ & $\begin{array}{l}\text { K. Sotiriadou } \\
\text { M. Skokou } \\
\text { A. Katrivanou }\end{array}$ \\
\hline $\begin{array}{l}\text { GREAT BRITAIN } \\
\text { Chairman: } \\
\text { Secretary: } \\
\text { Treasurer: }\end{array}$ & $\begin{array}{l}\text { H. Palazidou } \\
\text { K. Kasiakogia } \\
\text { P. Lekkos }\end{array}$ \\
\hline
\end{tabular}

SECTOR OF YOUNG PSYCHIATRISTS

Chairman: A. Economou

Secretary A: $\quad$ G. Pagkalos

Secretary B': $\quad$ A. Diakoumopoulou

UNION OF GREEK PSYCHIATRIC TRAINEES

Chairman: Th. Koutsomitros

Secretary: Ath. Antoniou

Treasurer: A. Mavromatos 
(લ€લ

KAA $\triangle$ OI

AYTOKATA

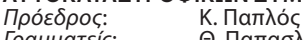

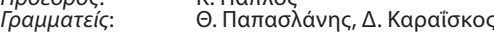

BIAISN $\Sigma Y M \Pi E P I \Phi O P \Omega N$

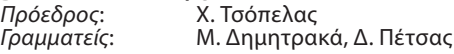

BIONOГIKH $\Psi$ YXIATPIKH $\Sigma$

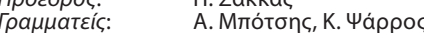

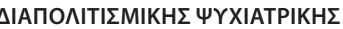

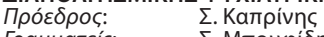

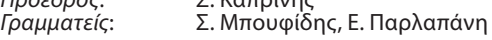

$\triangle$ IATAPAXE

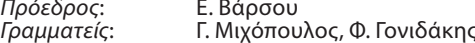

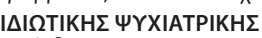

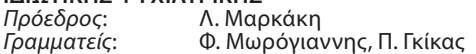

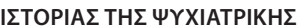

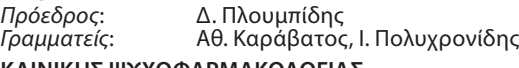

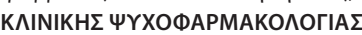

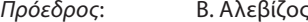

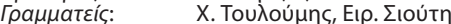

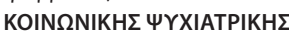

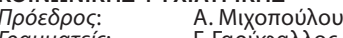

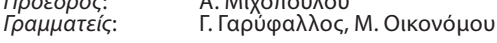

OV $\Sigma I O E E A P T H \Sigma E \Omega N$

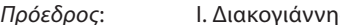

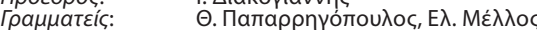

ПAI $\triangle$ YYXIATPIKH $\Sigma$

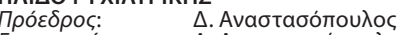

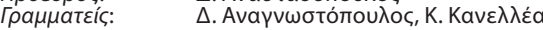

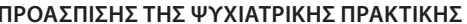

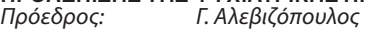

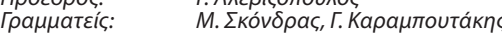

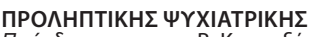

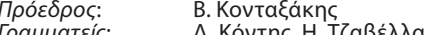

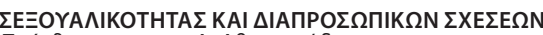

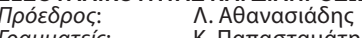

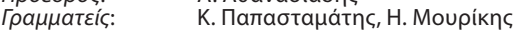

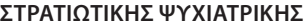

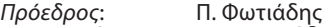

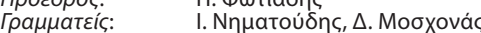

¿YMBOYAEYTIKH $\Sigma-\triangle I A \Sigma Y N \Delta E T I K H \Sigma$

UYXIATPIKH $\Sigma$ \& $\Psi$ YXO $\Sigma \Omega M A T I K H \Sigma$

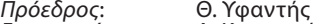

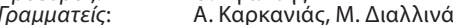

TEXNH $\Sigma \& \Psi Y X I A T P I K H \Sigma$

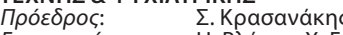

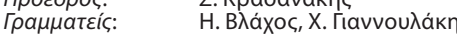

TH $А \Psi Y X I A T P I K H \Sigma$

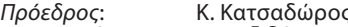

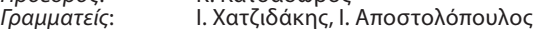

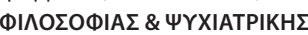

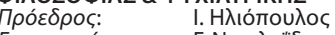

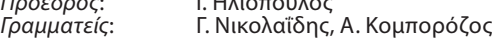

$\Psi \curlyvee X I A T P I K H \Sigma$ HOIKH $\Sigma$ \& $\triangle$ EONTO $\Lambda O \Pi A \Sigma$

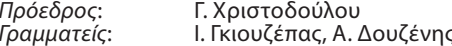

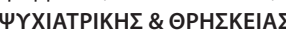

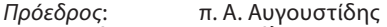

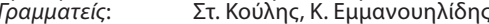

$\Psi$ YXIKH $\Sigma$ YГEIA $\Sigma$ ГYNAIK $\Omega N$ \&

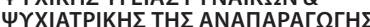

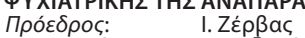

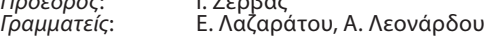

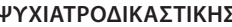

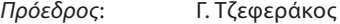

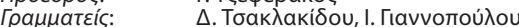

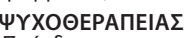

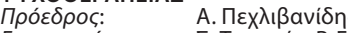

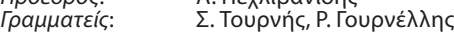

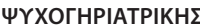

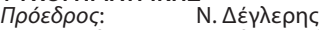

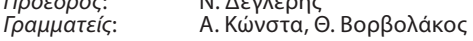

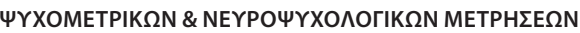

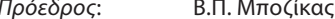

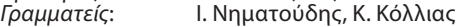

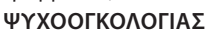

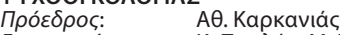

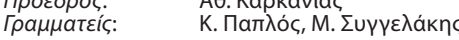

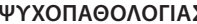

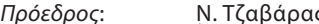

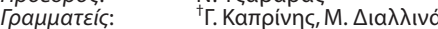

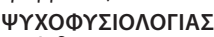

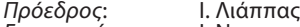

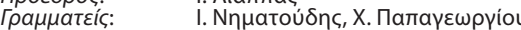

\section{SECTIONS}

SELF-DESTRUCTIVE BEHAVIORS

Chairman: $\quad$ K.Paplos

Secretaries: Th. Papaslanis, D. Karaiskos

VIOLENT BEHAVIORS

Chairman: $\quad$ Ch. Tsopelas

Secretaries: M. Dimitraka, D. Petsas

BIOLOGICAL PSYCHIATRY

Chairman: P.Sakkas

Secretaries: $\quad$ A. Botsis, C. Psarros

CROSS-CULTURAL PSYCHIATRY

Chairman: $\quad S$. Kaprinis

Secretaries: $\quad$ S. Boufidis, H. Parlapani

EATING DISORDERS

Secretaries: J. Michopoulos, F. Gonidakis

PRIVATE PRACTICE PSYCHIATRY

Chairman: L. Markaki

Secretaries: F. Morogiannis, P. Gkikas

HISTORY OF PSYCHIATRY

$\begin{array}{ll}\text { Chairman: } & \text { D. Ploumpidis } \\ \text { Secretaries: } & \text { Ath. Karavatos, J. Polyhronidis }\end{array}$

PSYCHOPHARMACOLOGY

Chairman: $\quad$ V. Alevizos

Secretaries: C. Touloumis, I. Siouti

SOCIAL PSYCHIATRY

Chairman: A. Michopoulou

Secretaries: G. Garyfallos, M. Economou

SUBSTANCE ABUSE

J.Diakoyiannis

Secretaries: Th. Paparrigopoulos, El. Mellos

CHILD PSYCHIATRY

Chairman: $\quad$ D. Anastasopoulos

Secretaries: D. Anagnostopoulos, K. Kanellea

ADVOCACY OF PSYCHIATRIC PRACTICE

Chairman: G. Alevizopoulos

Secretaries: M.Skondras, G. Karampoutakis

PREVENTIVE PSYCHIATRY

Chairman: V. Kontaxakis

Secretaries: D. Kontis, E. Tzavellas

SEXUALITY AND INTERPERSONAL RELATIONSHIPS

Chairman: $\quad$ L. Athanasiadis

Secretaries: $\quad$ K. Papastamatis, H. Mourikis

MILITARY PSYCHIATRY

Chairman:

Secretaries: J. Nimatoudis, D. Moschonas

CONSULTATION-LIAISON PSYCHIATRY

\& PSYCHOSOMATICS

Chairman: $\quad$ T. Hyphantis

Secretaries: A. Karkanias, M. Diallina

ART \& PSYCHIATRY

Chairman: S. Krasanakis

Secretaries: $\quad$ E. Vlachos, C. Giannoulaki

TELEPSYCHIATRY

Chairman: K. Katsadoros

Secretaries: J.Chatzidakis, J. Apostolopoulos

PHILOSOPHY \& PSYCHIATRY

Chairman: J.lliopoulos

Secretaries: $\quad$ G. Nikolaidis, A. Komborozos

PSYCHIATRY \& ETHICS

Chairman: G. Christodoulou

Secretaries: J.Giouzepas, A. Douzenis

PSYCHIATRY \& RELIGION

Chairman: $\quad$ r. A. Avgoustidis

Secretaries: $\quad$ S. Koulis, K. Emmanouilidis

WOMEN'S MENTAL HEALTH \&

REPRODUCTIVE PSYCHIATRY

Chairman: $\quad J$ Zervas

Secretaries: H. Lazaratou, A. Leonardou

FORENSIC PSYCHIATRY

G. Tzeferakos

Secretaries: $\quad$ D. Tsaklakidou, J. Giannopoulou

PSYCHOTHERAPY

Chairman: A. Pechlivanidis

Secretaries: S. Tournis, R. Gournellis

PSYCHOGERIATRICS

Chairman: N. Degleris

Secretaries: $\quad$ A. Konsta, Th. Vorvolakos

PSYCHOMETRIC \& NEUROPSYCHOLOGICAL MEASUREMENTS

Chairman: $\quad$ V.P. Bozikas

Secretaries: J. Nimatoudis, K. Kollias

PSYCHO-ONCOLOGY

Chairman: A. Karkanias

Secretaries: $\quad$ K. Paplos, M. Syngelakis

PSYCHOPATHOLOGY

Chairman: N. Tzavaras

Secretaries: $\quad{ }^{\dagger} \mathrm{G}$. Kaprinis, M. Diallina

PSYCHOPHYSIOLOGY

Chairman: J. Liappas

Secretaries: J. Nimatoudis, C. Papageorgiou 


\section{(46)}

\section{YYXIATPIKH}

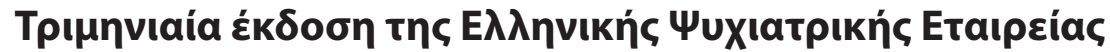

\section{TEPIEXOMENA}

\section{Eıठıı́́ áp $\theta \rho a$}

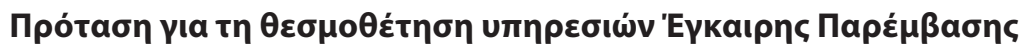

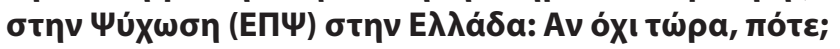

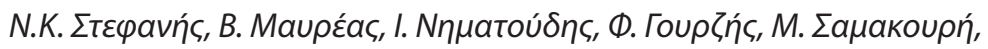

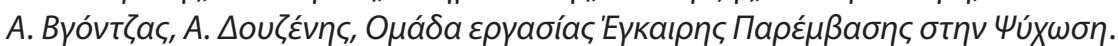

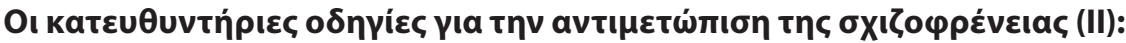

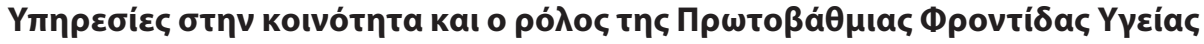

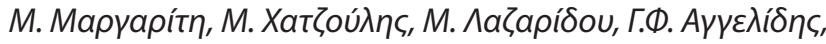

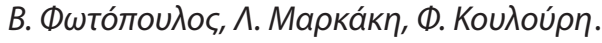

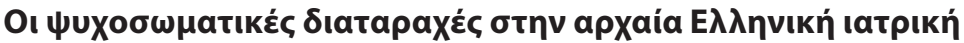

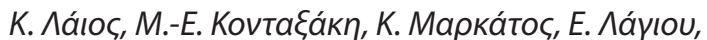

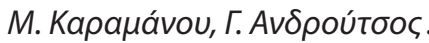

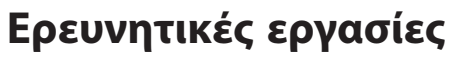

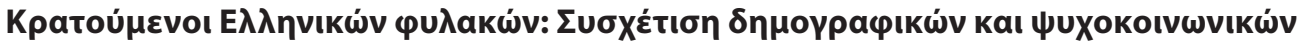

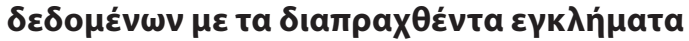

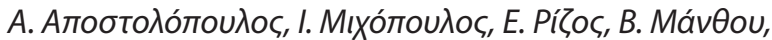

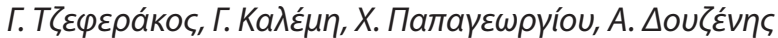

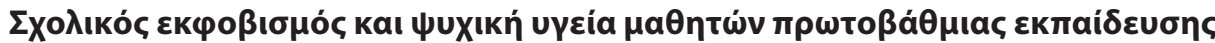

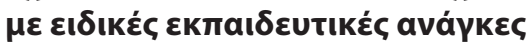

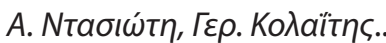

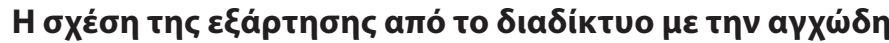
каเ катаӨ

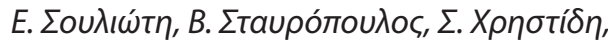

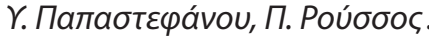

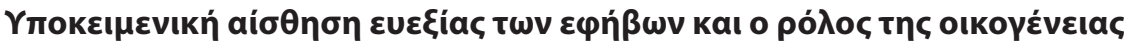

АІк. Аантротои́'оo 


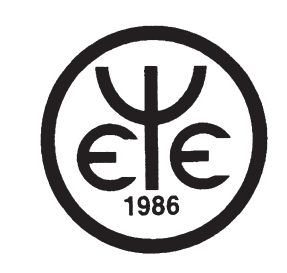

PSYCHIATRIKI

\section{Quarterly journal published by the Hellenic Psychiatric Association}

\section{CONTENTS}

\section{Special articles}

A proposal for the implementation of Early Intervention in Psychosis (EIP) services in Greece: If not now, when?

N.C. Stefanis, V. Mavreas, I. Nimatoudis, F. Gourzis, M. Sarakouri,

A. Vgontzas, A. Douzenis, EIP Working group .

Clinical guidelines for the management of schizophrenia (II):

Community service-level interventions and the role of Primary Care

M. Margariti, M. Hadjulis, M. Lazaridou, G.F. Angelidis,

V. Fotopoulos, L. Markaki, F. Koulouri.

Psychosomatic disorders in ancient Greek medicine

K. Laios, M.-I. Kontaxaki, K. Markatos, E. Lagiou,

M. Karamanou, G. Androutsos.

\section{Research articles}

Prisoners in Greek prisons: Correlation of demographic and psychosocial data with committed crimes

A. Apostolopoulos, I. Michopoulos, E.M. Rizos, V. Manthou,

G. Tzeferakos, G. Kalemi, Ch. Papageorgiou, A. Douzenis

Bullying and the mental health of schoolchildren with special educational needs in primary education

A. Dasioti, G. Kolaitis.

The relationship of internet addiction with anxiety

and depressive symptomatology

E. Soulioti, V. Stavropoulos, S. Christidi,

Y. Papastefanou, P. Roussos.

The role of the family in adolescents' subjective well-being

A. Lampropoulou. 


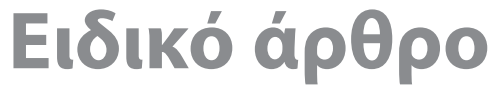 Special article
}

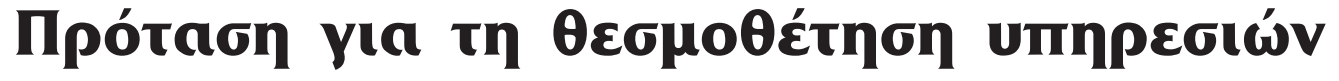

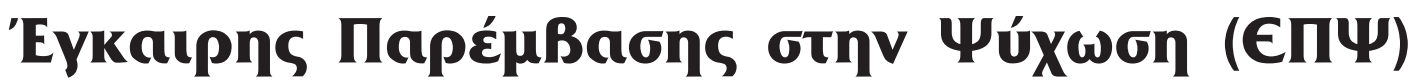 otnv €ลतáda:

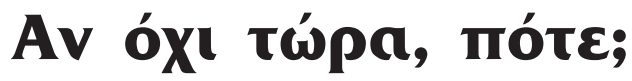

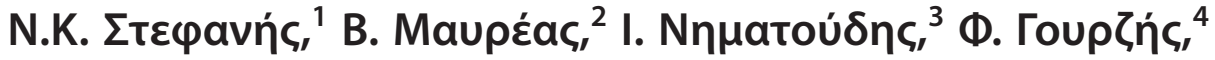

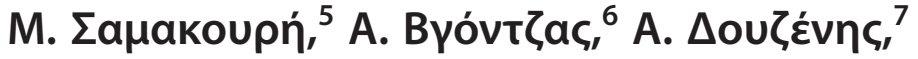

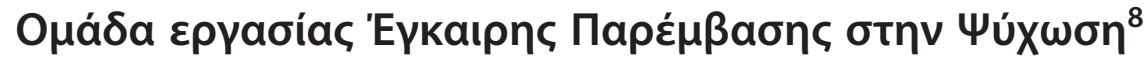

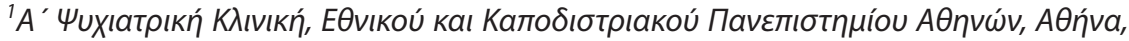

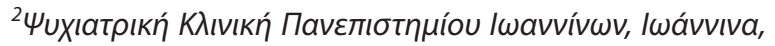

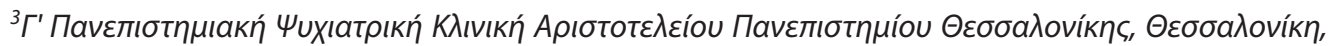

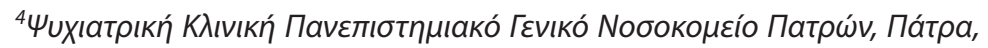

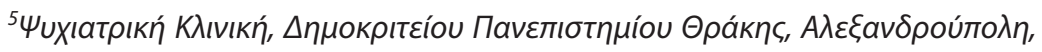

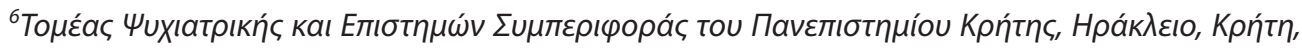

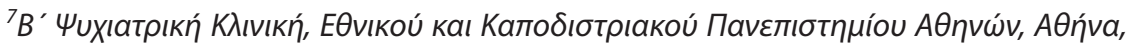

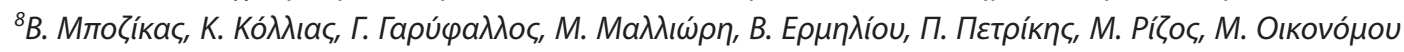

Uuxıatpıkń 2018, 29:107-117

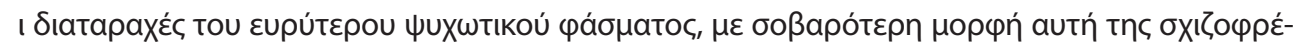

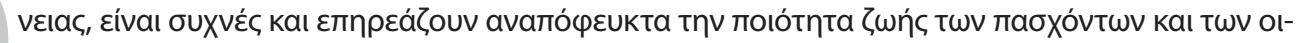

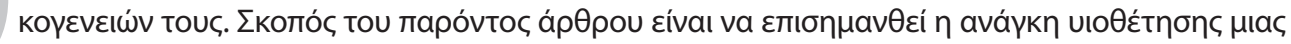

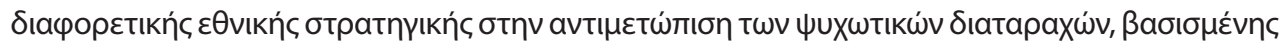

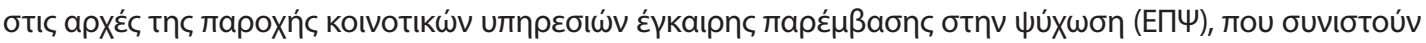

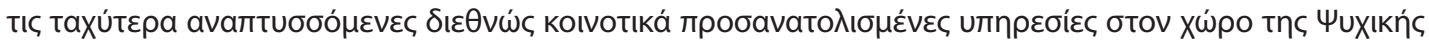

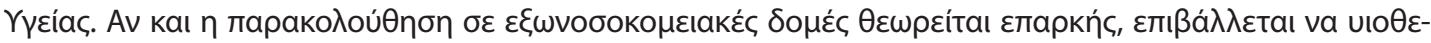

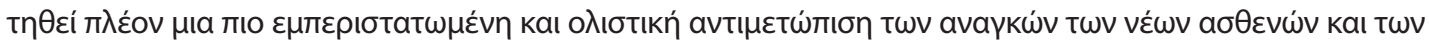

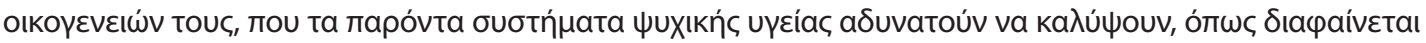

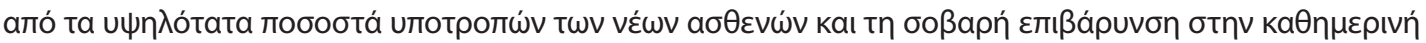

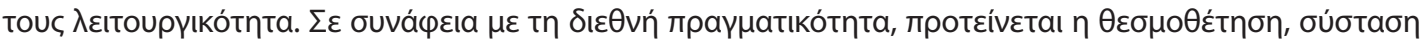

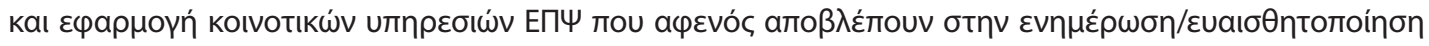




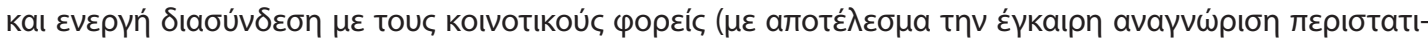

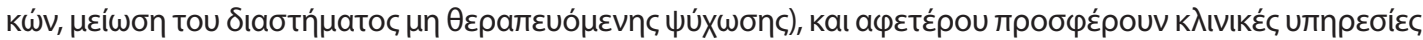

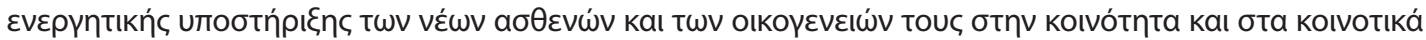

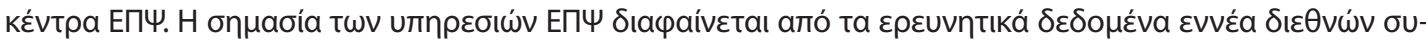

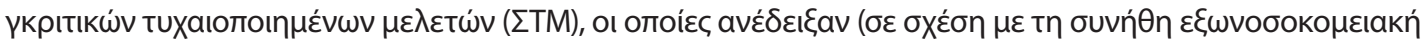

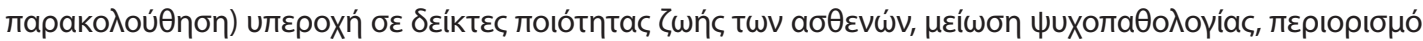

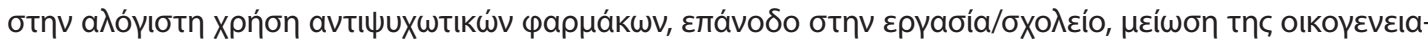

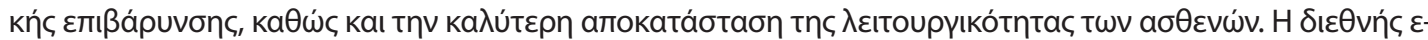

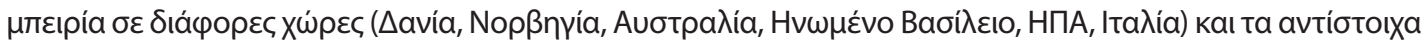

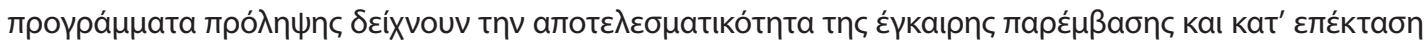

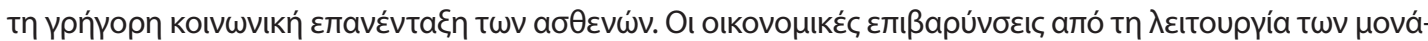

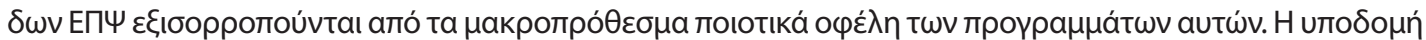

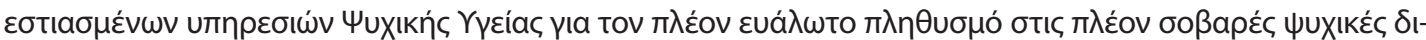

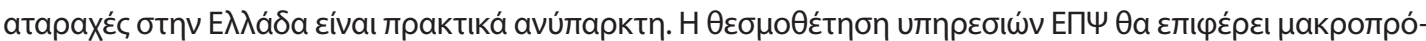

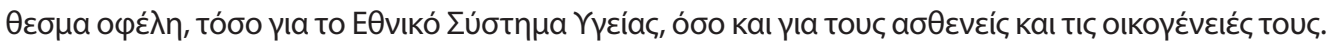

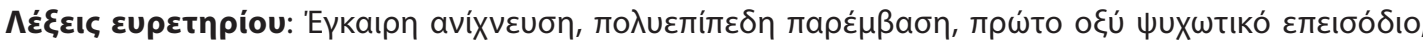

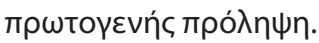

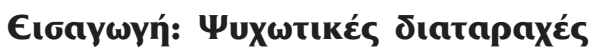

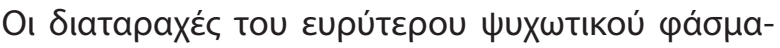

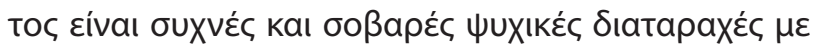

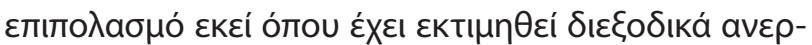

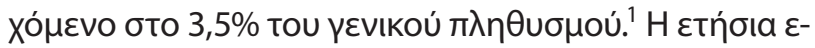

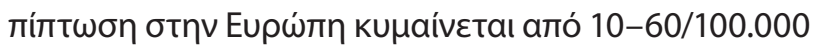

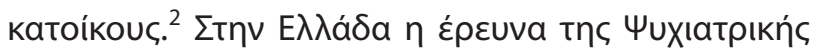

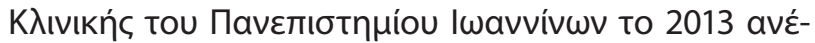

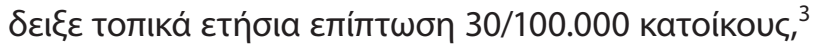

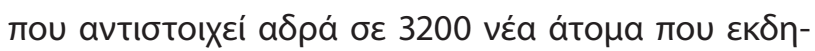

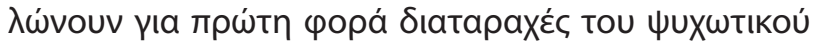

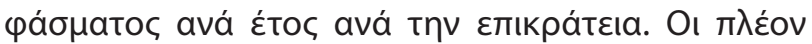

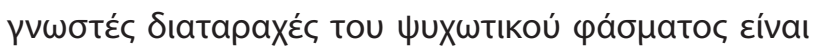

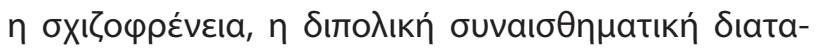

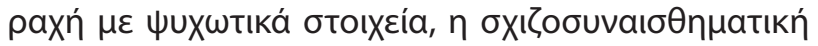

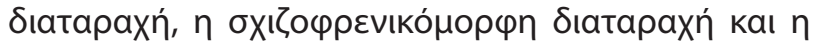

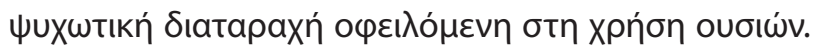

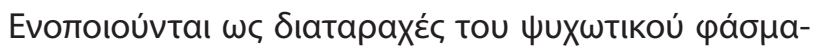

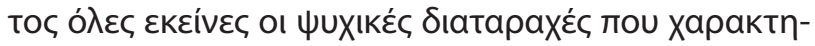

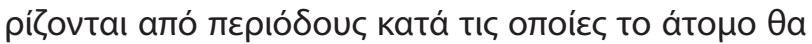

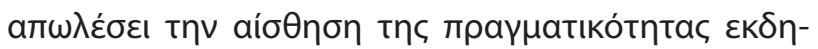

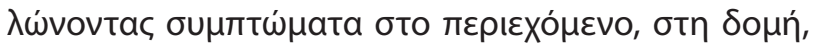

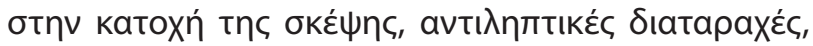

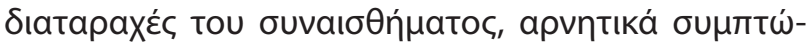

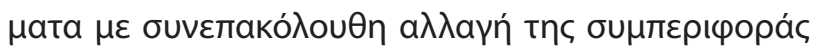

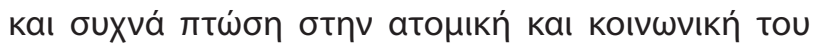

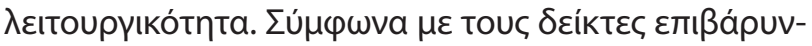

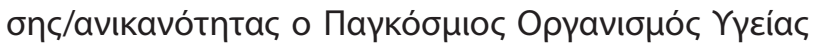

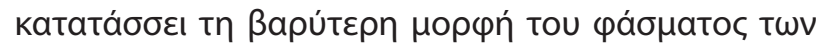

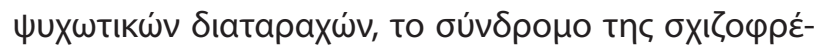

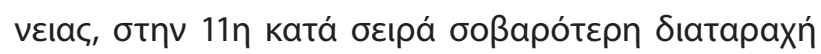

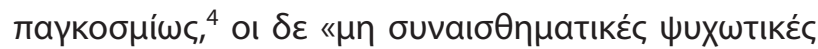

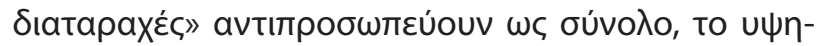

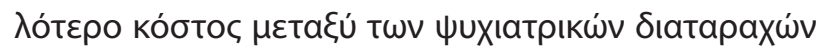

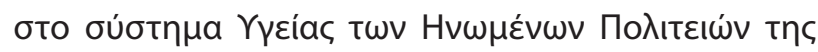

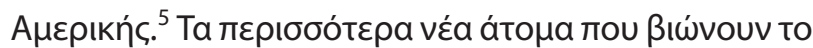

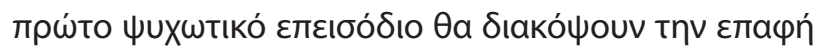

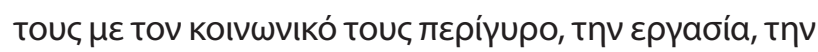

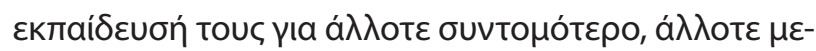

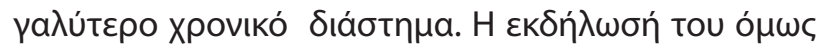

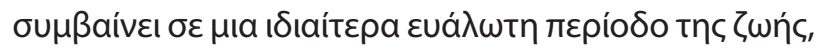

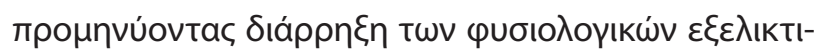

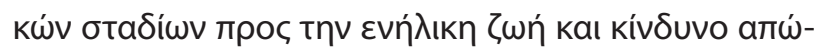

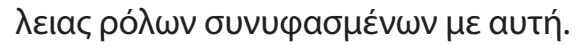

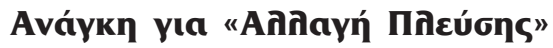

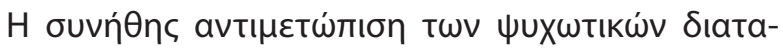

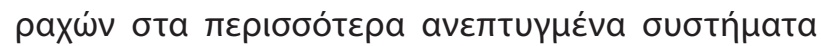

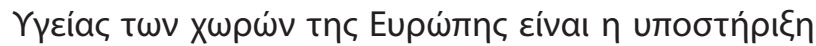

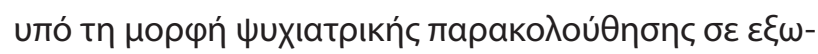

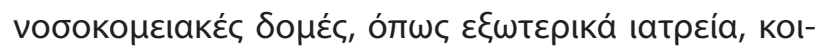

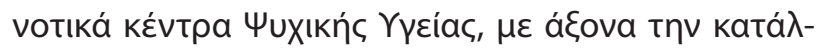

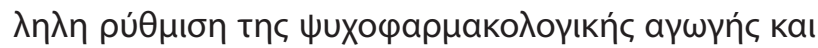

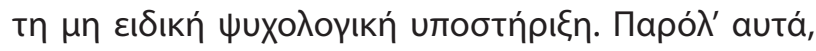

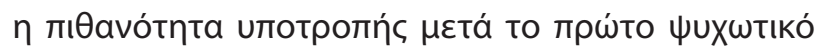

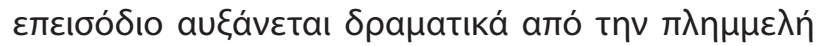




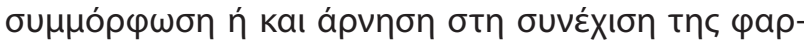

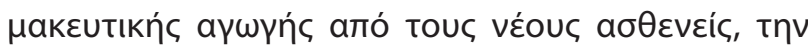

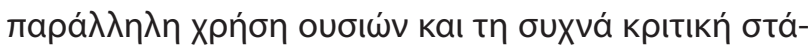

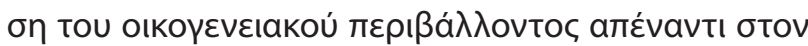

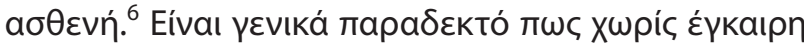

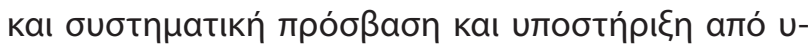

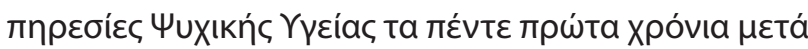

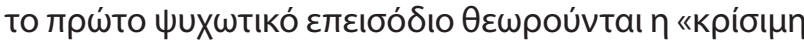

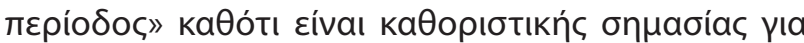

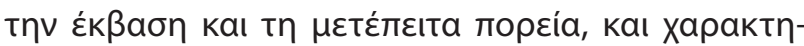

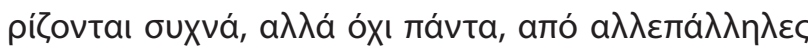

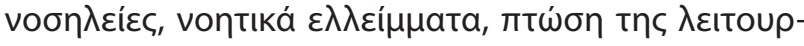

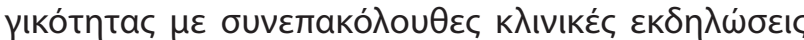

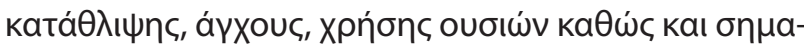

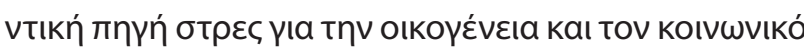

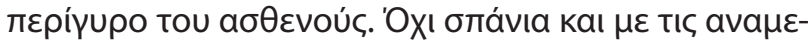

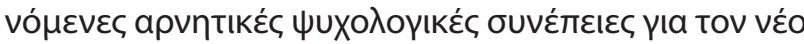

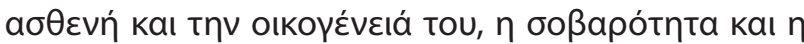

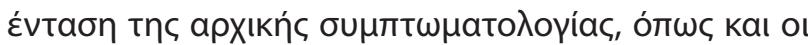

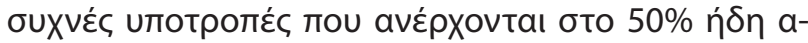

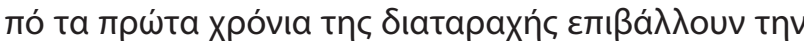

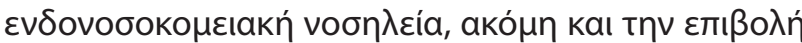

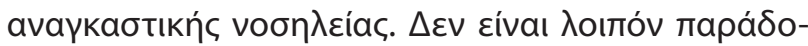

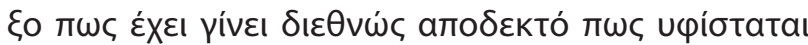

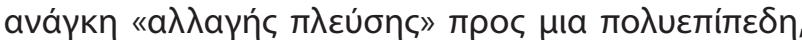

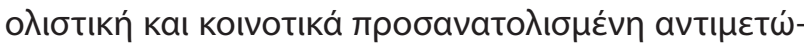

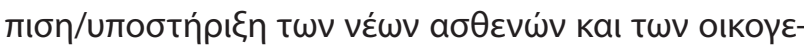

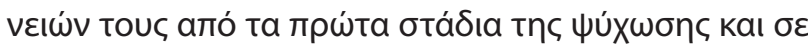

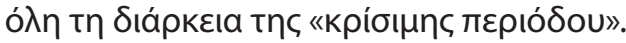

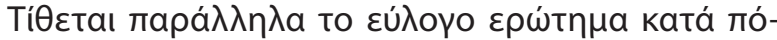

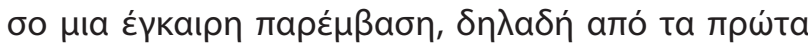

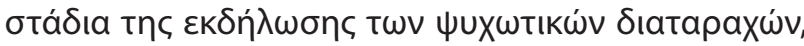

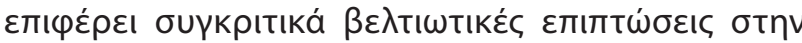

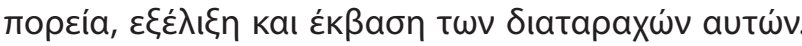

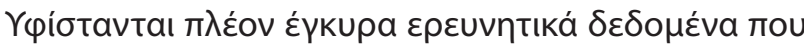

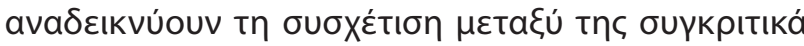

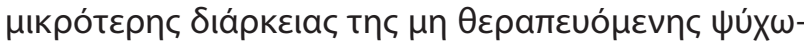

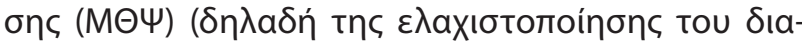

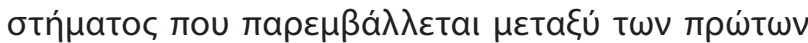

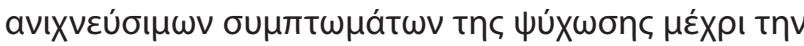

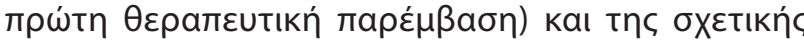
$\beta \varepsilon \lambda t i ́ \omega \sigma \eta \varsigma$ a

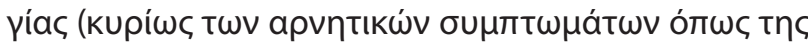

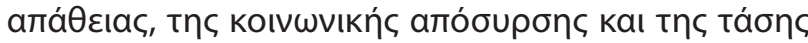

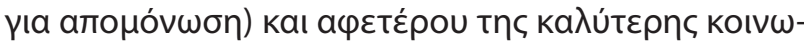

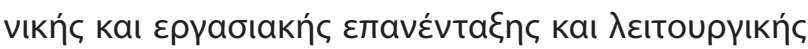

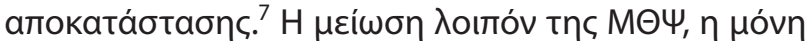

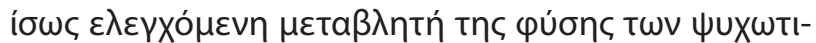

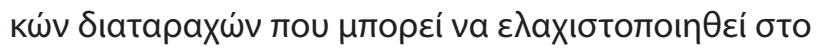

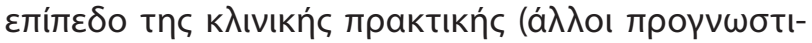

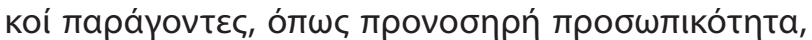

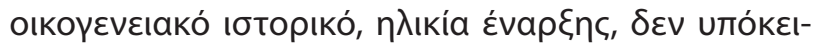

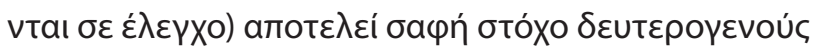

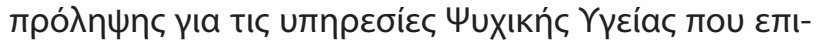

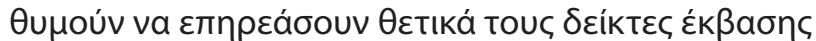

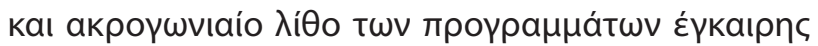

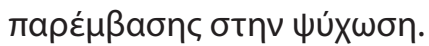

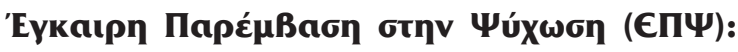 ApXés Kaı กียเToupyía}

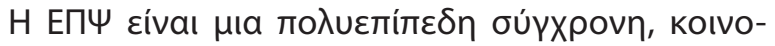

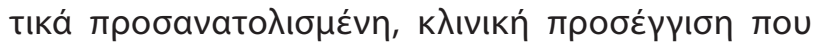

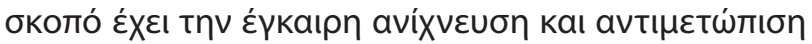

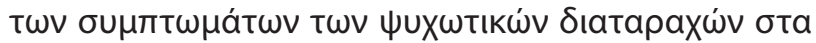

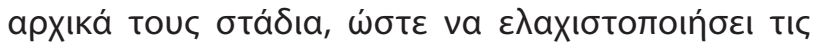

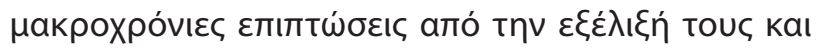

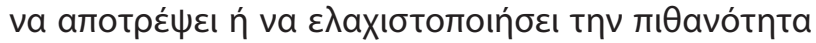

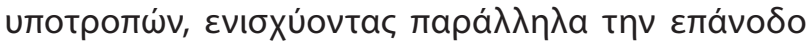

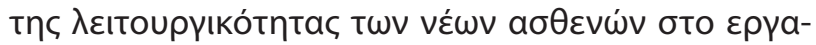

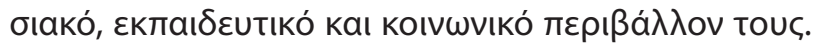

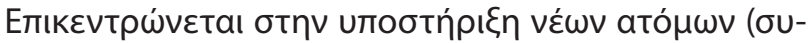

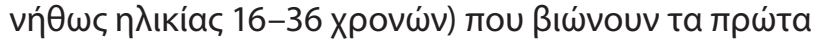

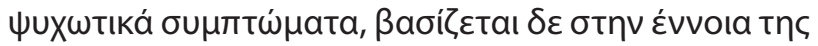

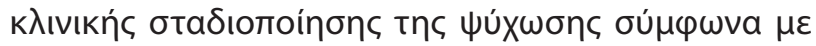

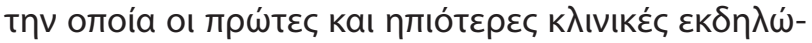

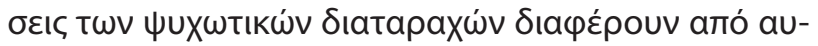

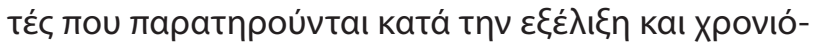

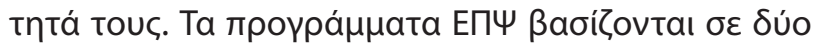

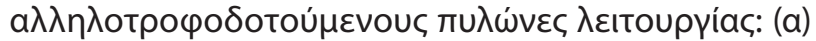

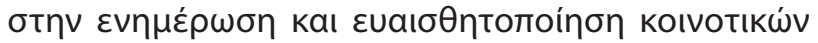

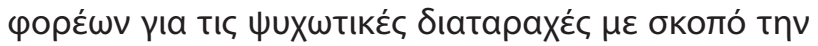

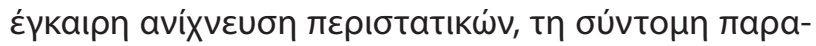
по

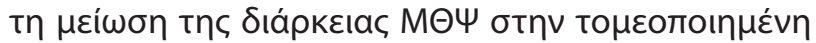

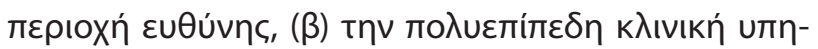

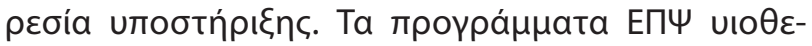

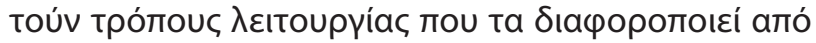

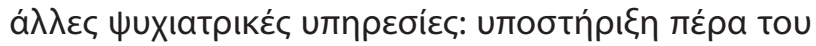

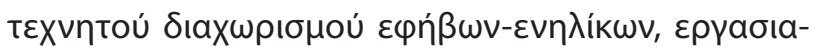

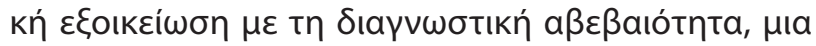

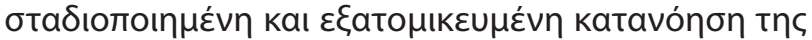

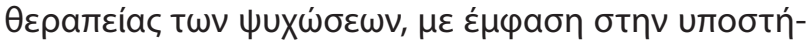




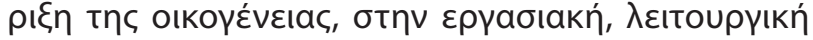

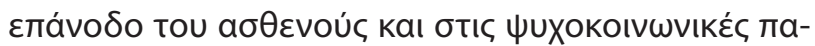

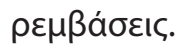

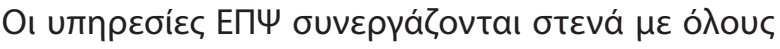

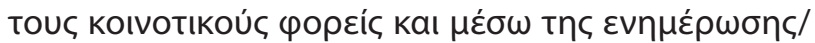

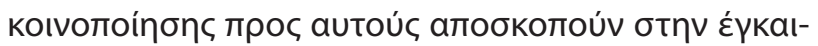

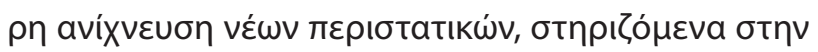

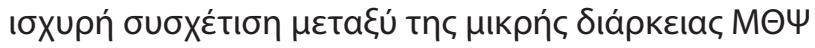

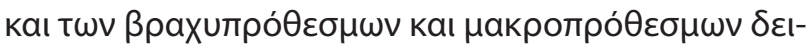

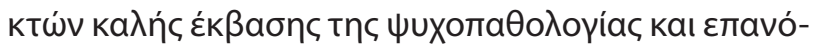

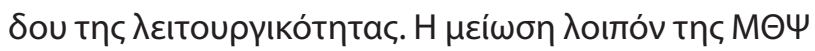

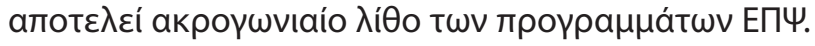

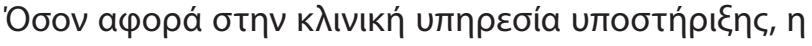

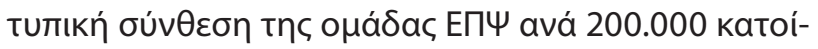

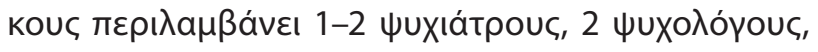

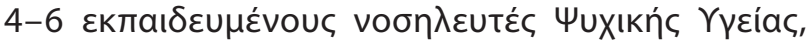

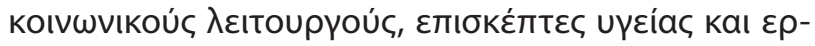

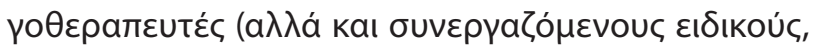

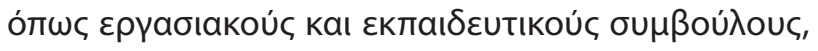

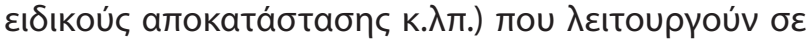

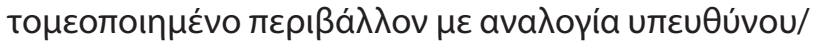

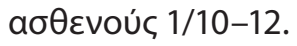

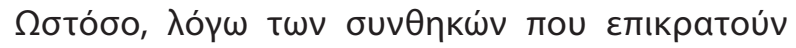

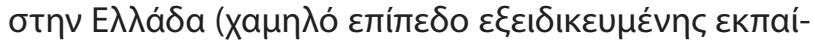

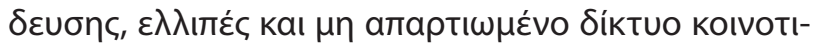

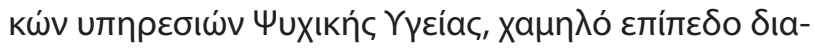

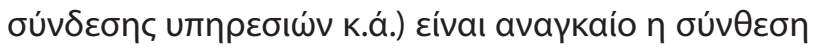

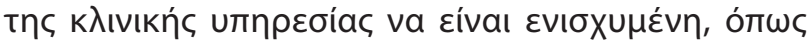

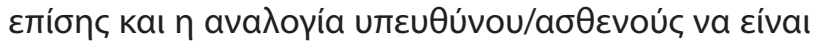

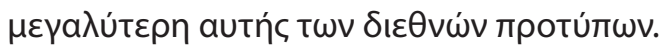

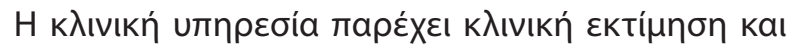

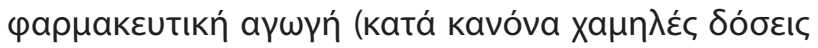

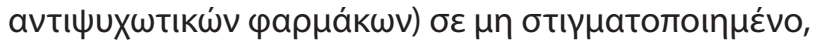

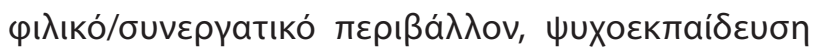

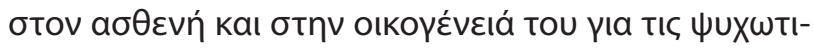

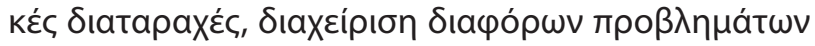

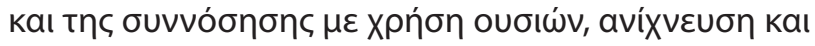

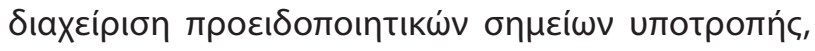

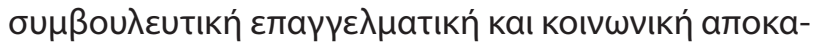

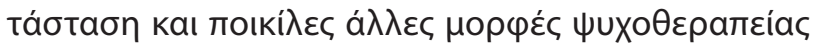

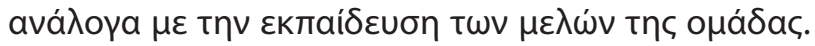

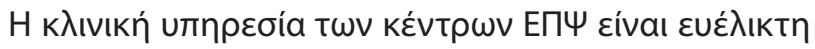

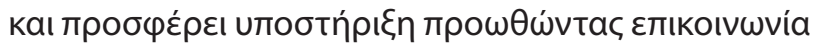

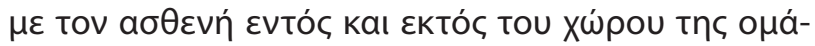

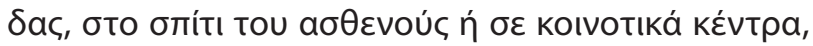

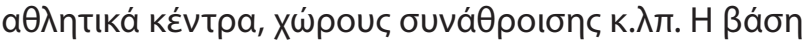

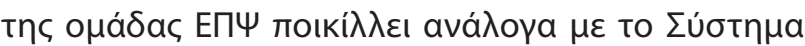

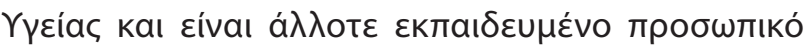

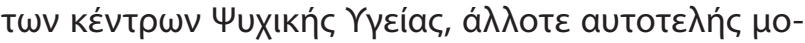

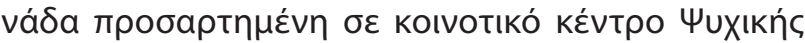

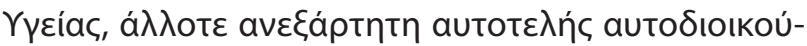

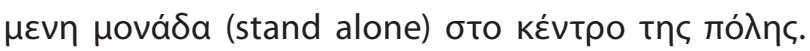

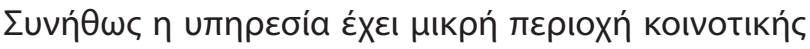

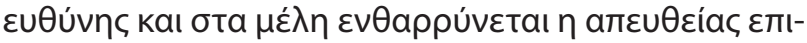

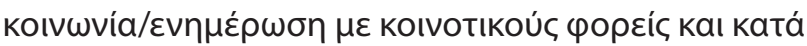

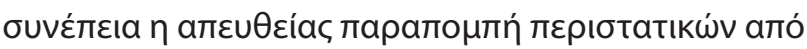

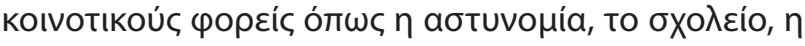

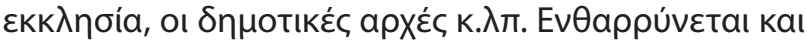

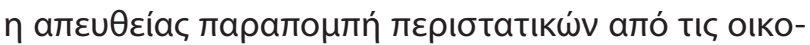

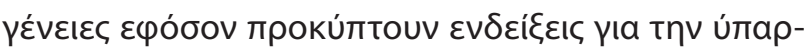

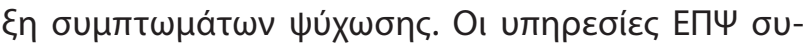

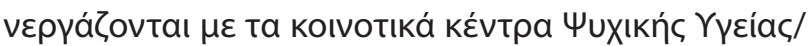

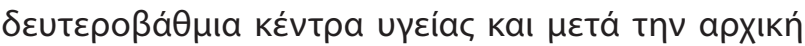

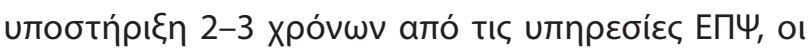

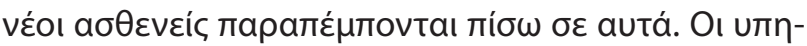

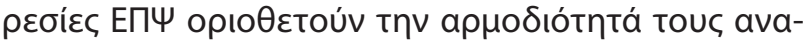

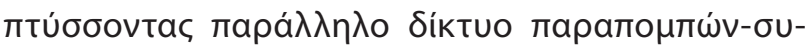

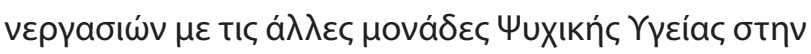

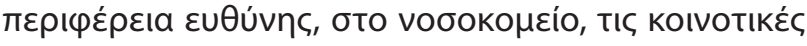

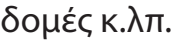

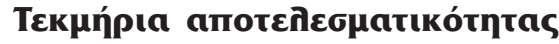

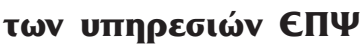

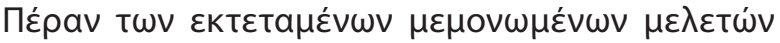

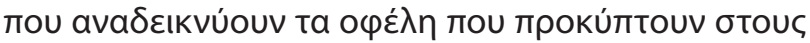

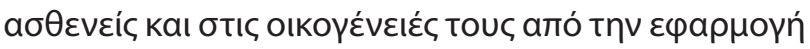

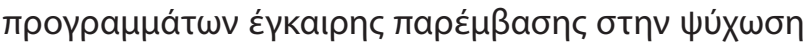

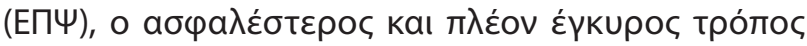

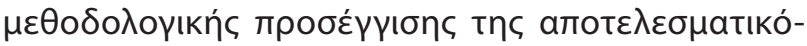

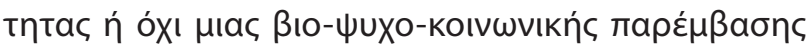

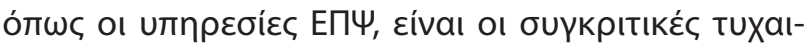

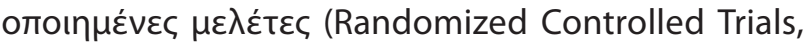

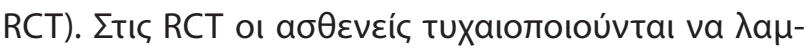

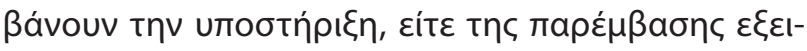

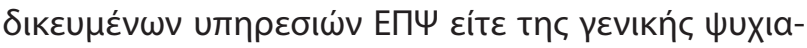

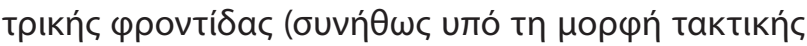

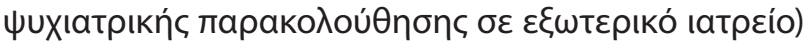

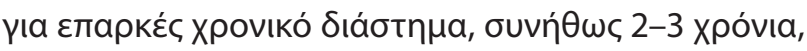

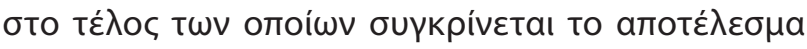

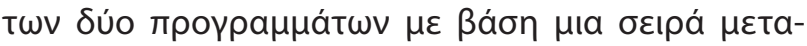

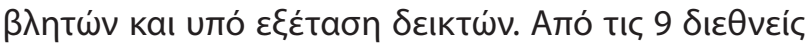




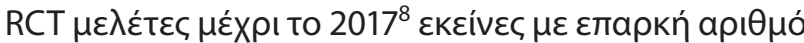

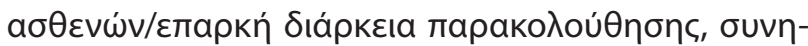

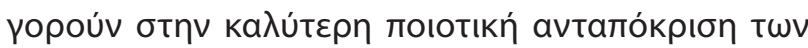

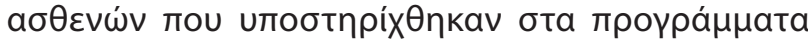

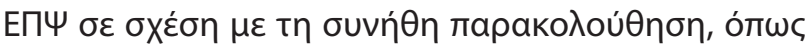

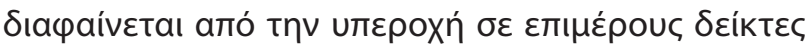
$\tau \omega v$ RCT $\mu \varepsilon \lambda \varepsilon \tau \omega ́ v$ :

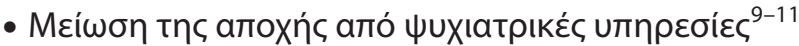

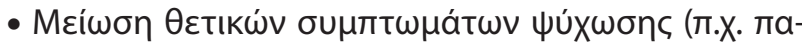

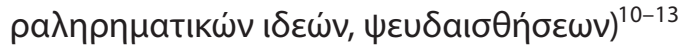

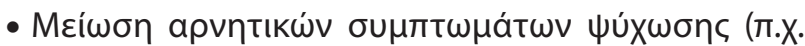

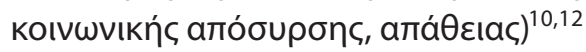

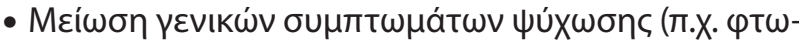

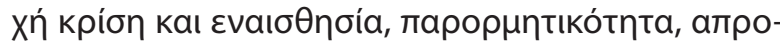

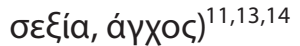

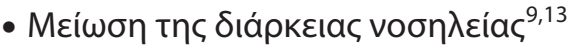

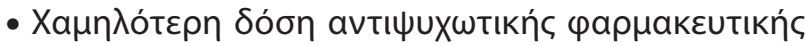
aүwүńs $\varsigma^{10}$

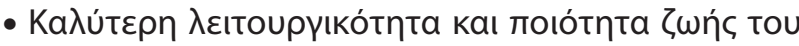

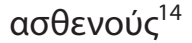

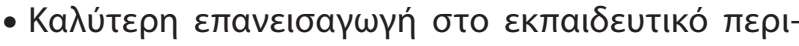

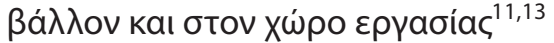

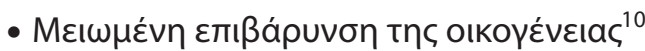

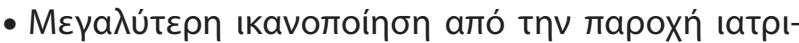

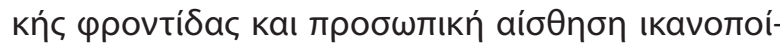
nonc. ${ }^{10,11,14}$

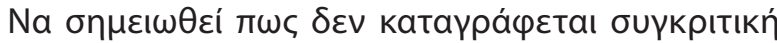

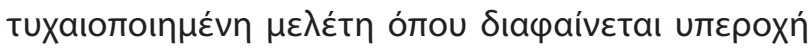

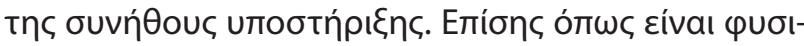

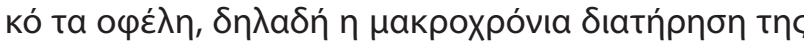

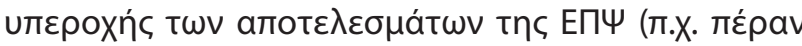

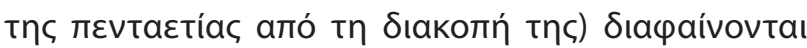

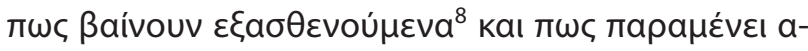

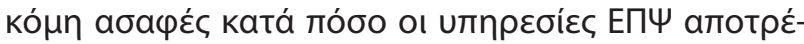

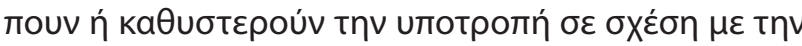

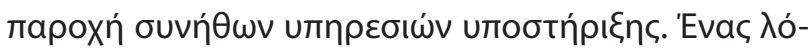

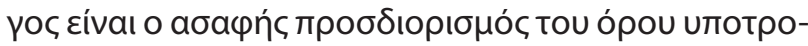

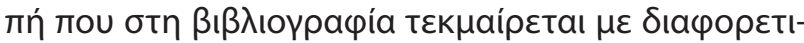

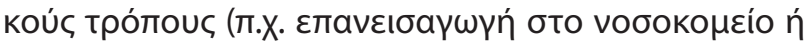

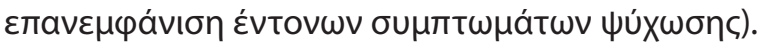

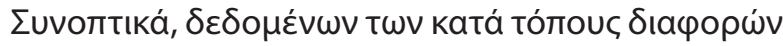

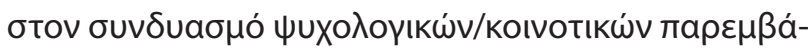

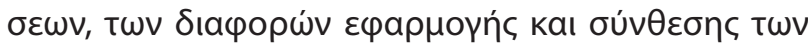

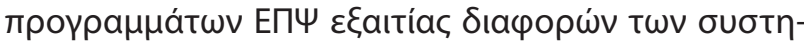

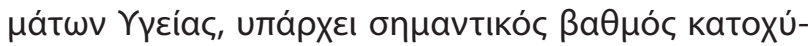

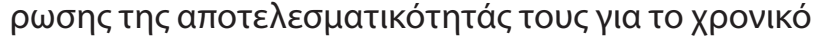

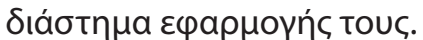

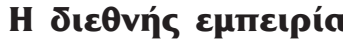

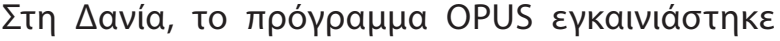

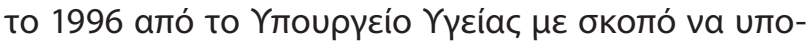

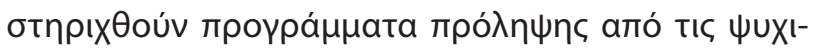

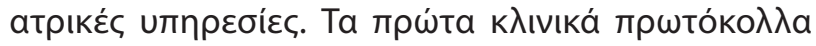

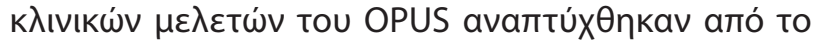

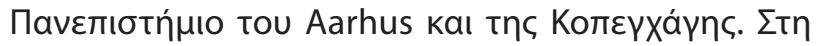

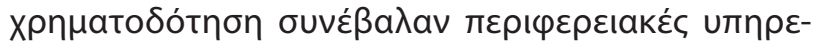

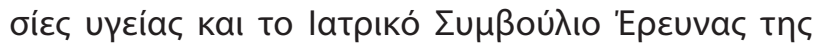

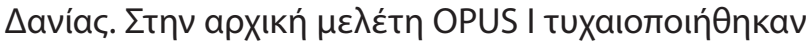

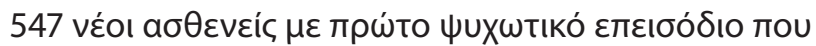

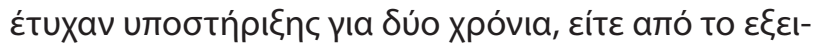

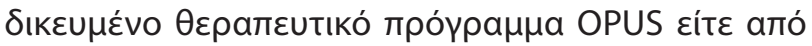

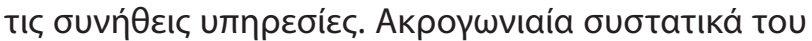

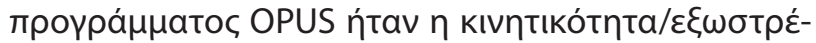

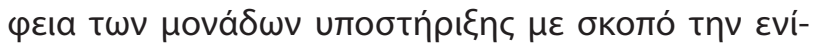

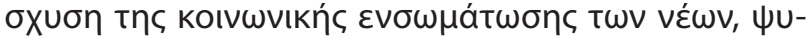

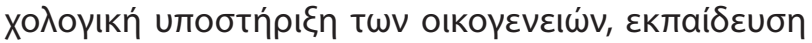

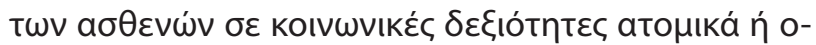

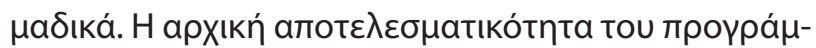

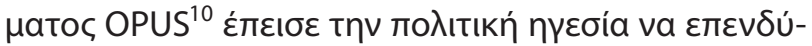

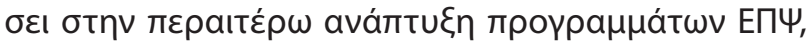

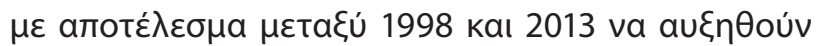

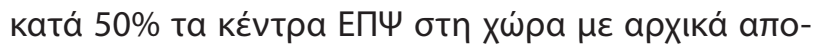

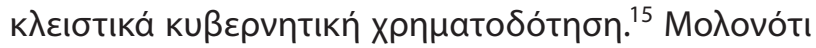

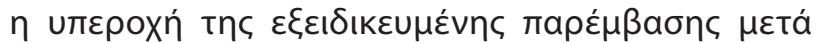

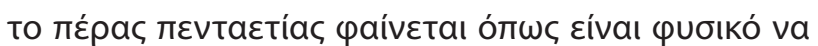

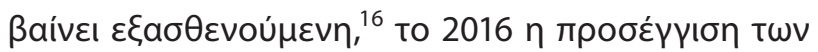

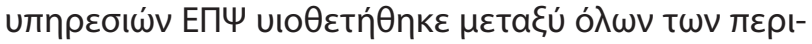

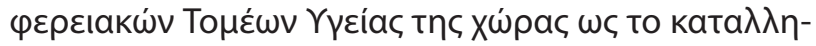

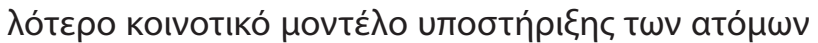

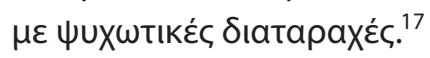

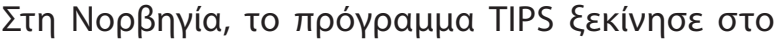

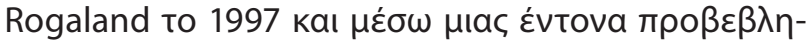

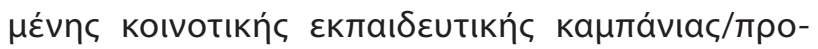

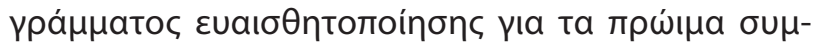

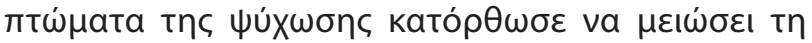

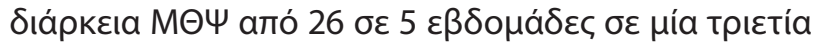

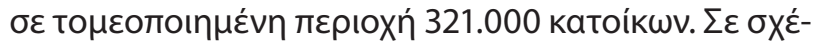

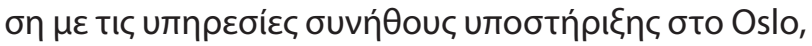

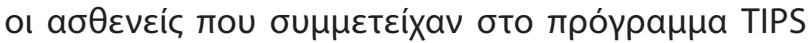

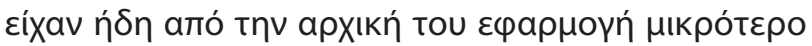




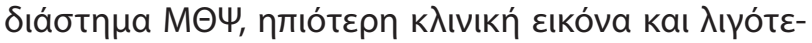

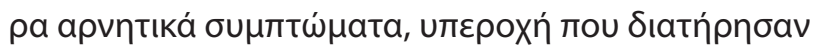

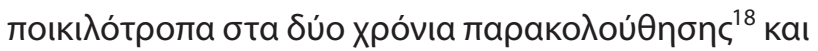

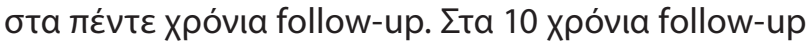

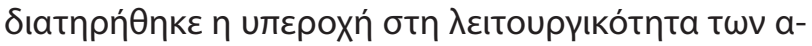

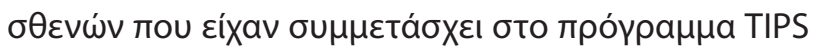

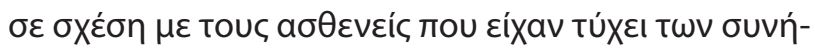

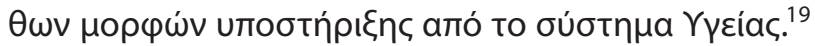

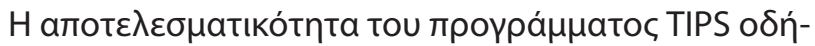

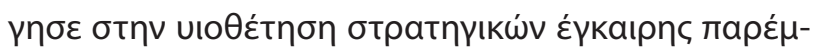

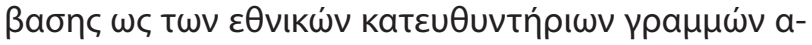

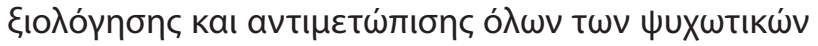

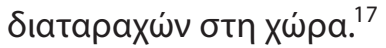

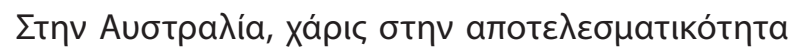

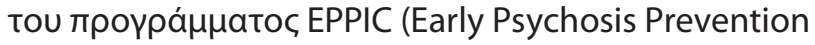

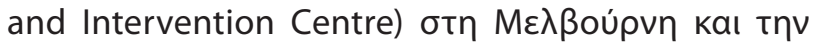

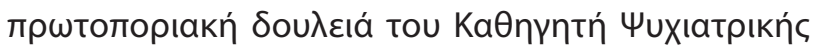

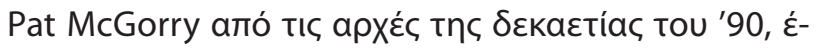

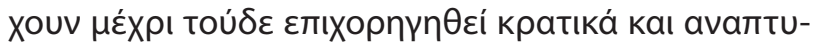

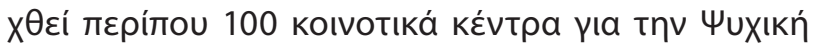

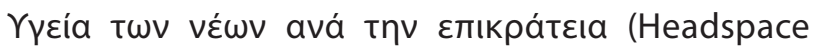
National Youth Mental Health Foundation).

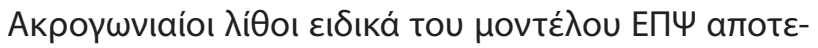

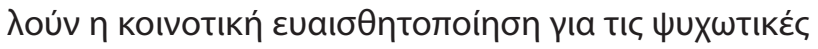

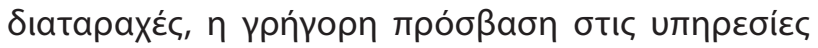

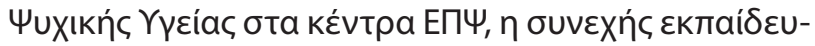

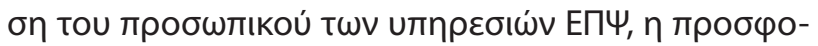

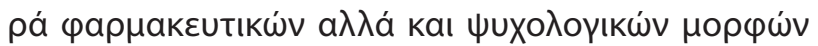

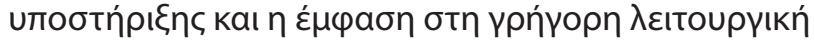

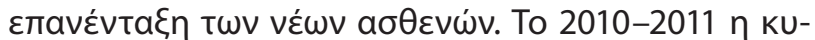

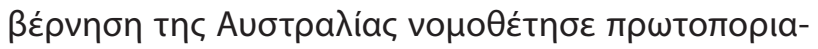

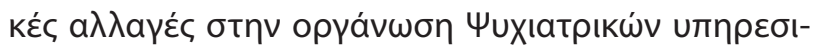

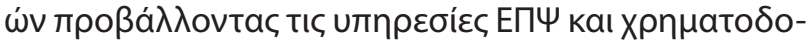

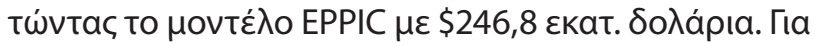

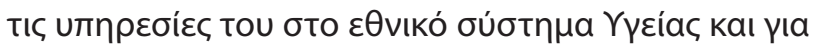

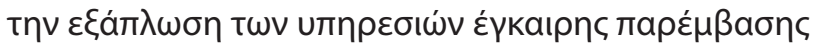

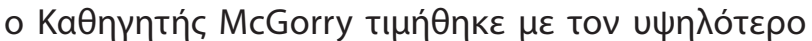

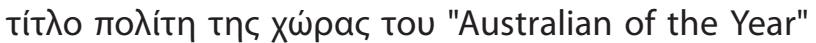
to 2010.

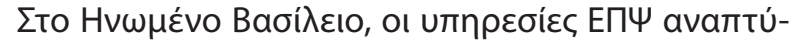

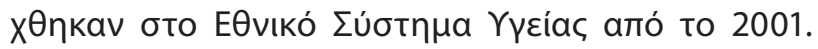

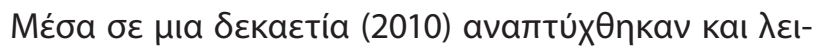

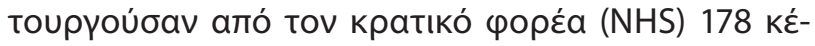

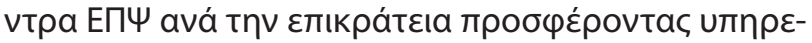

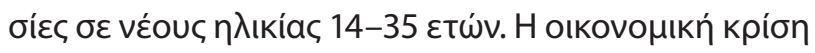

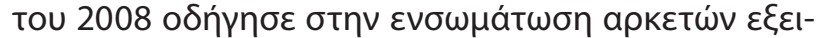

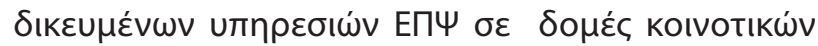

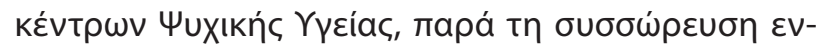

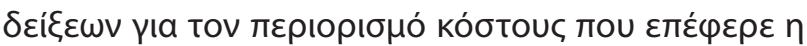

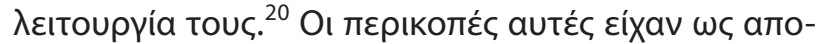

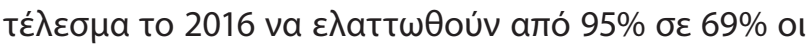

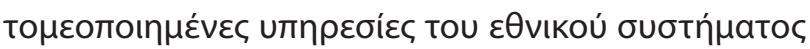

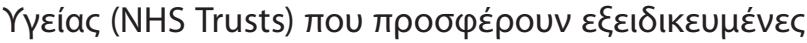

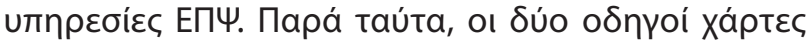
2014 (CG178) кaı 2016 tou EӨvıкоú Ivotıtoútou yıa

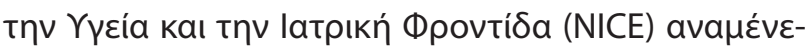

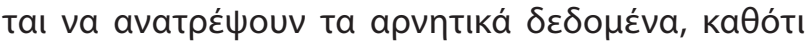

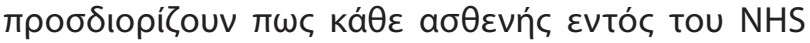

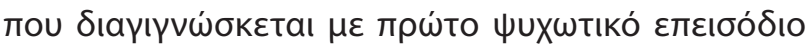

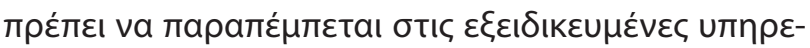

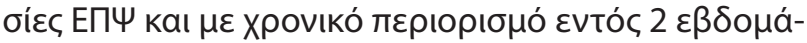

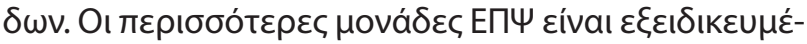

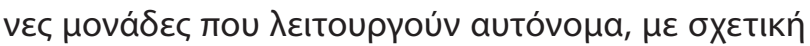

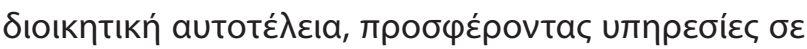

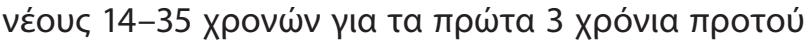

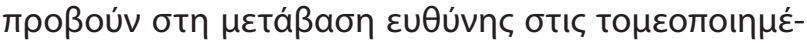

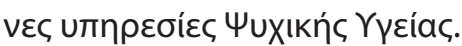

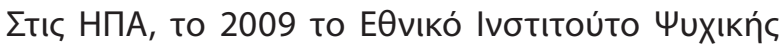

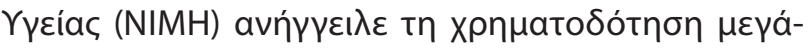

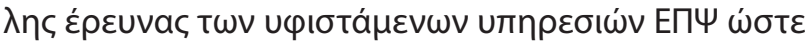

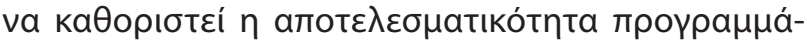

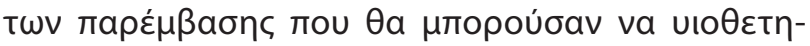

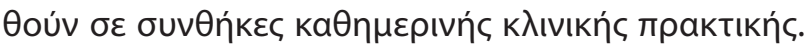

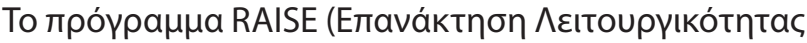

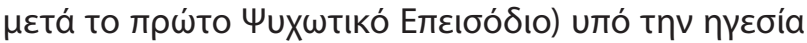

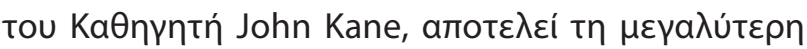

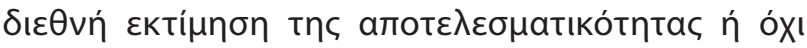

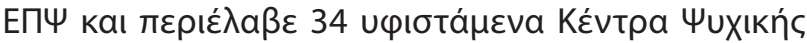

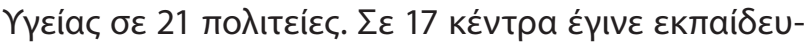

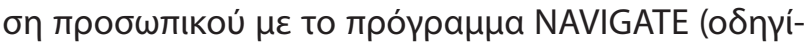

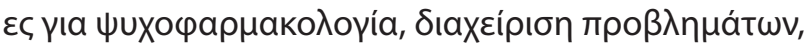

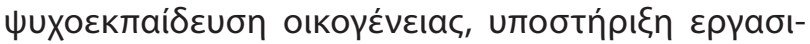

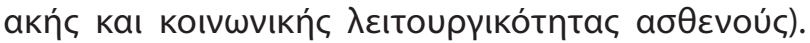

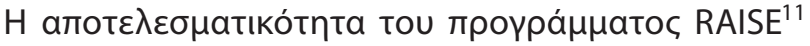

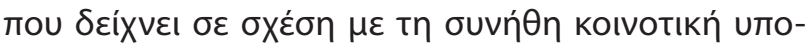

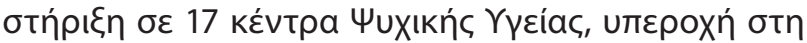

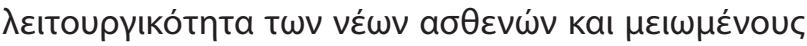

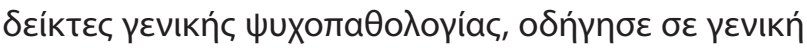

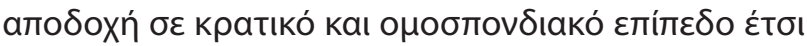

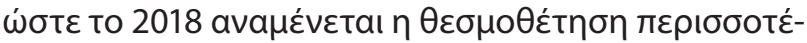

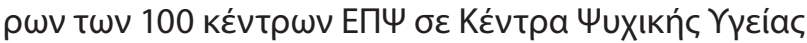




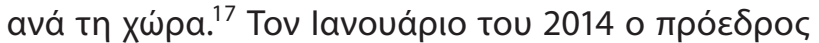

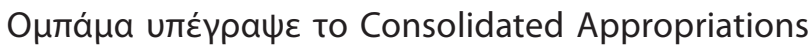

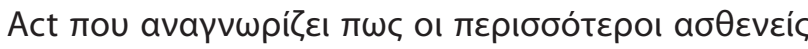

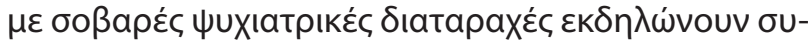

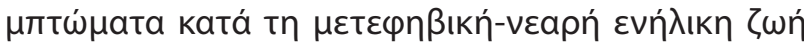

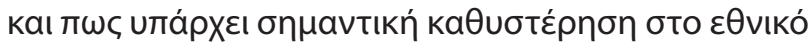

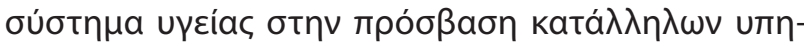

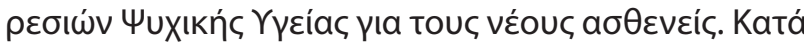

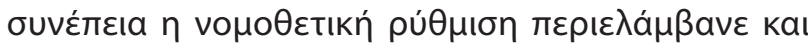

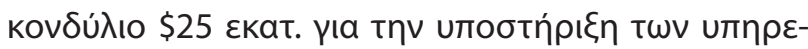

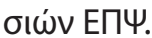

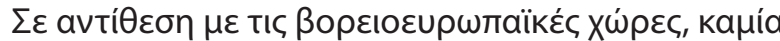

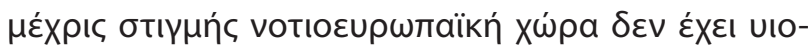

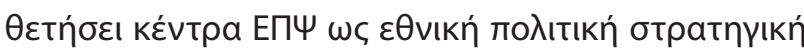

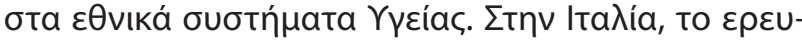

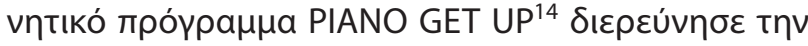

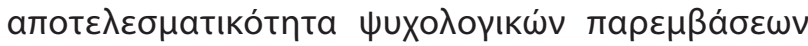

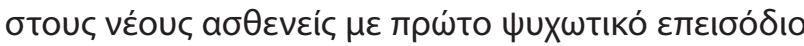

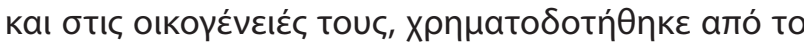

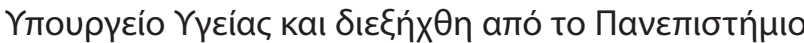

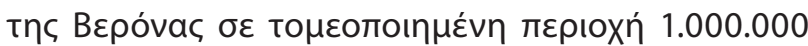

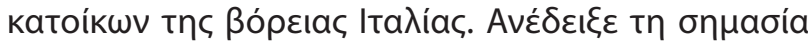

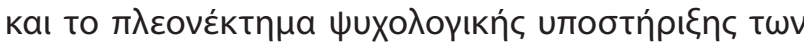

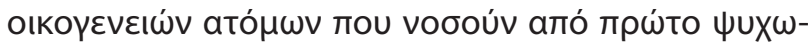

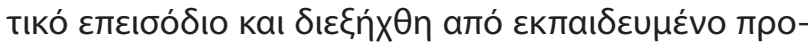

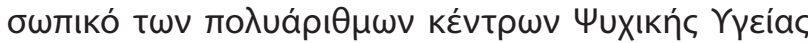

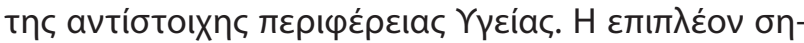

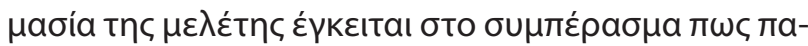

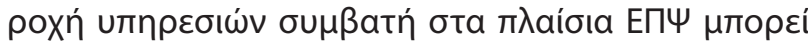

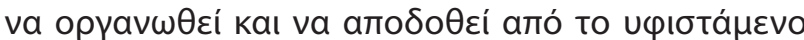

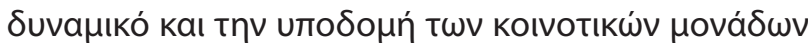

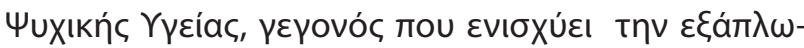

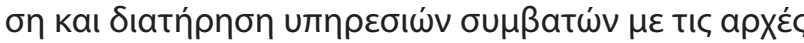

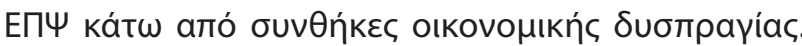

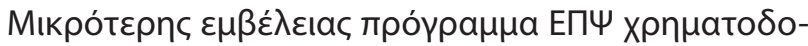

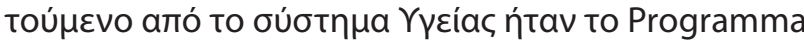

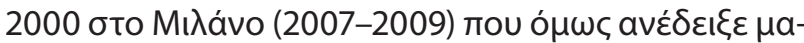

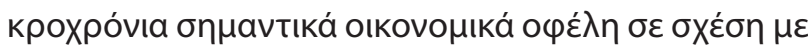

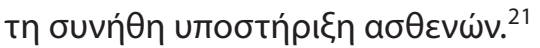

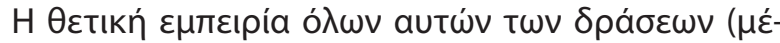

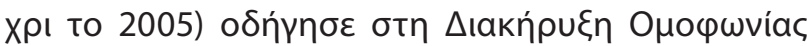

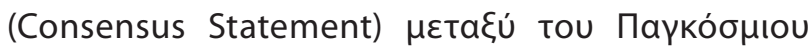

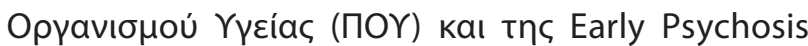
Association yıa tпv ЕП $\Psi^{22} \mu \varepsilon$ тпv отоі́a о ПОҮ ито-

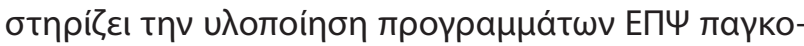

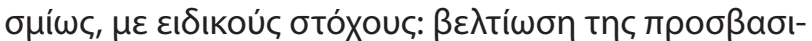

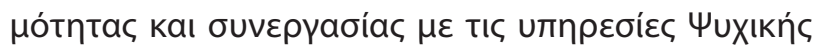

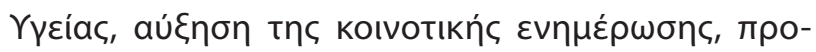

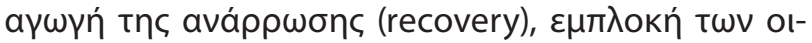

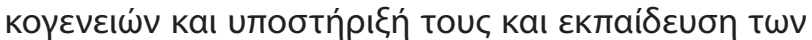

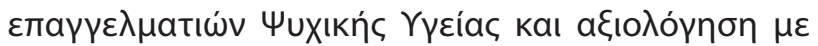

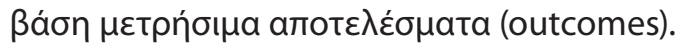

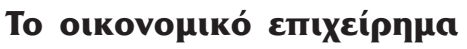

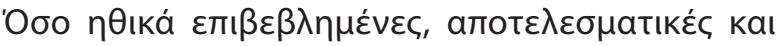

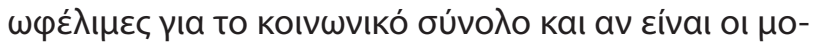

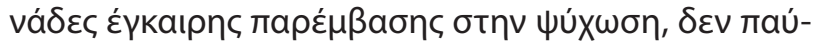

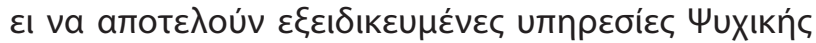

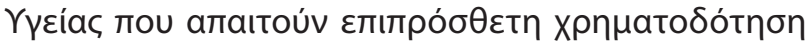

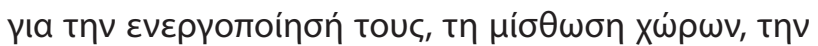

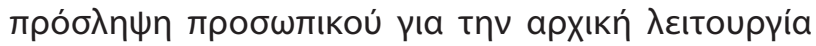

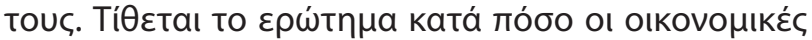

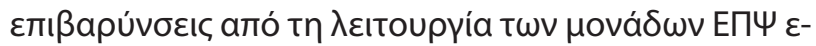

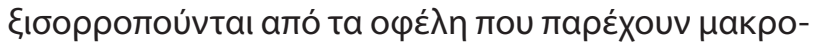

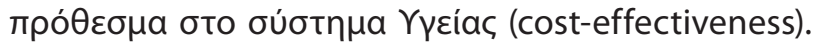

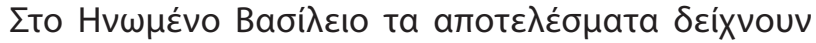

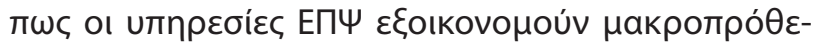

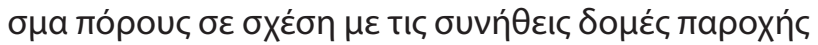

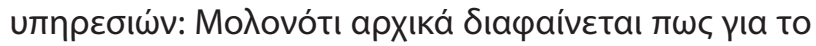

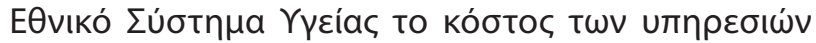

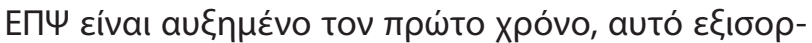

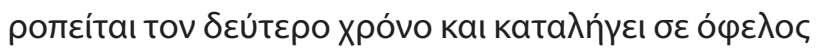

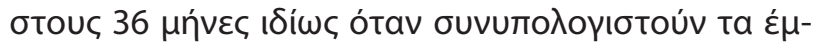

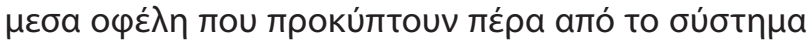

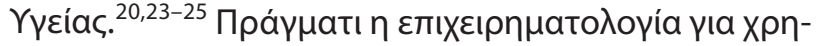

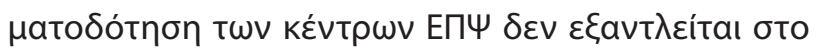

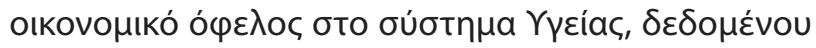

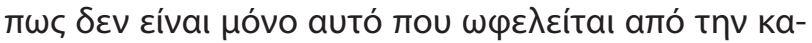

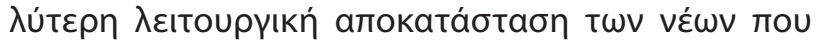

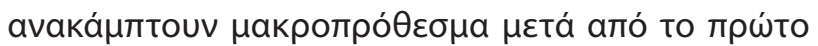

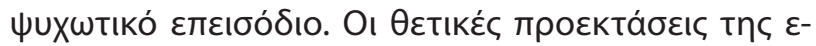

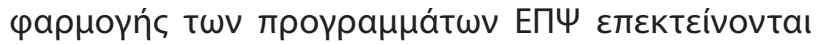

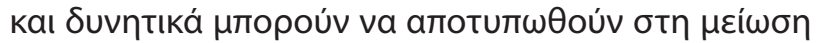

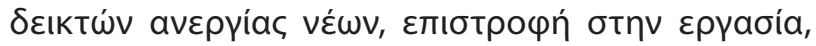

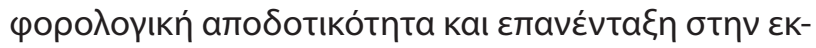

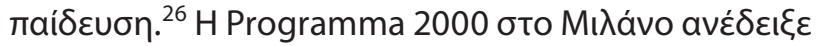

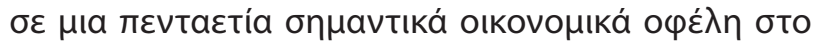

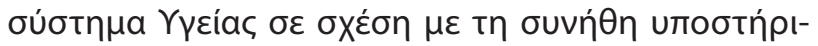

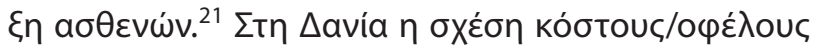

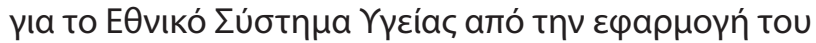

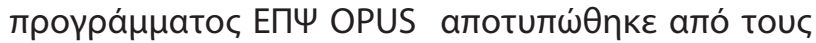




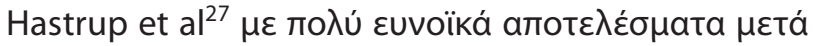

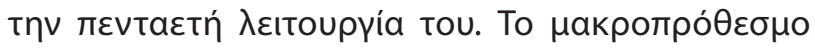

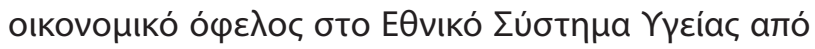

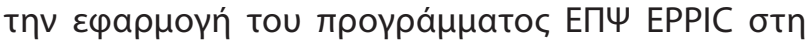

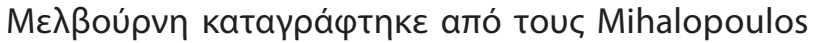

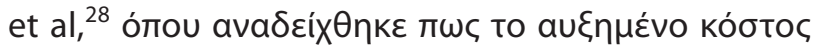

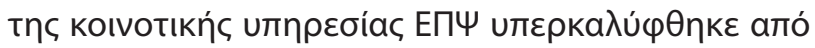

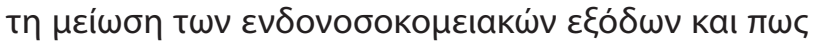

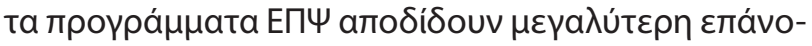

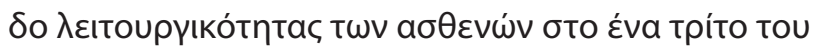

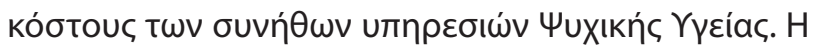

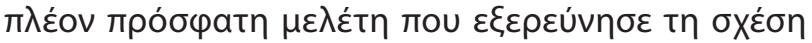

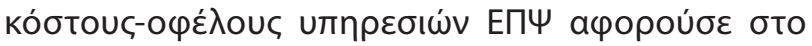

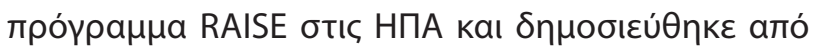

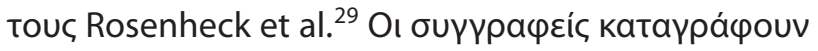

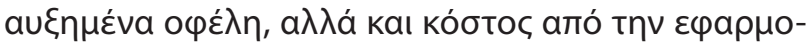

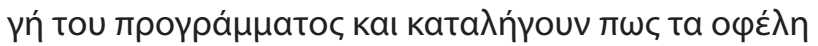

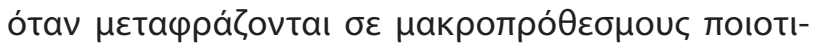

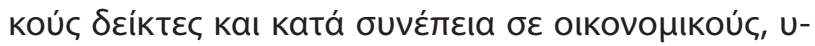

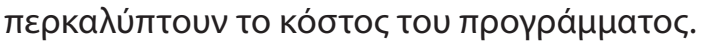

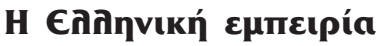

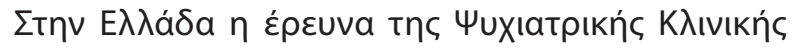

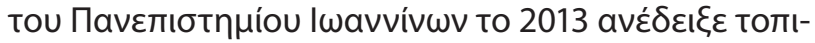

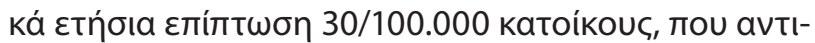

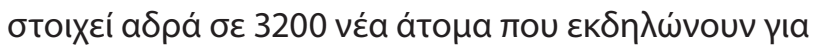

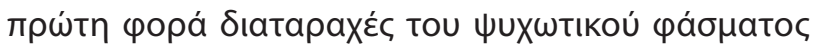

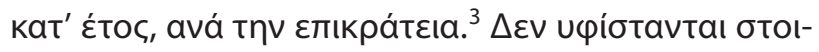

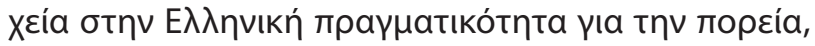

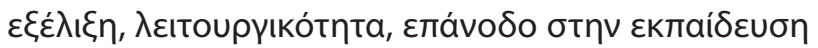

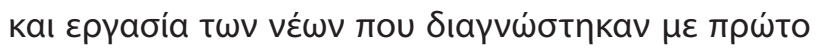

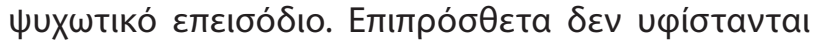

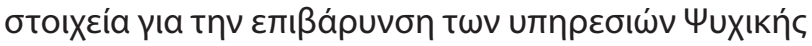

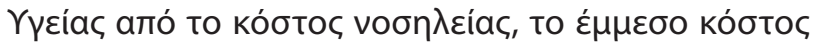

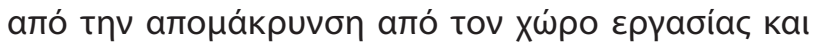

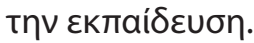

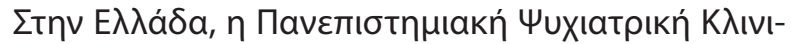

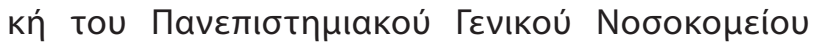

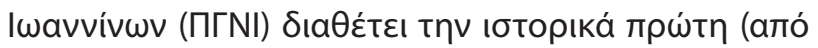

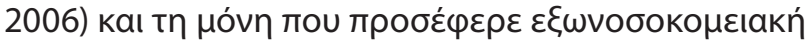

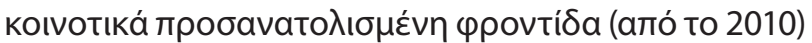

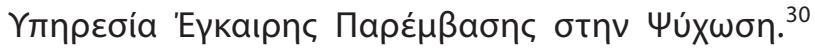

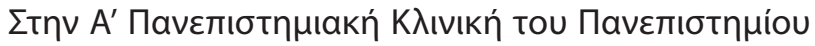

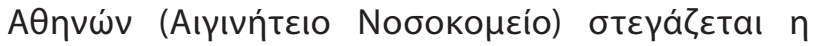

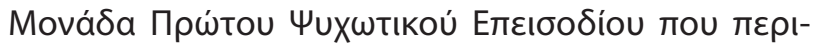

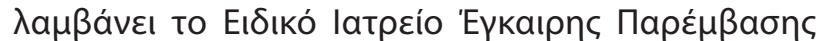

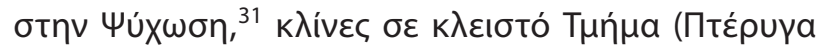

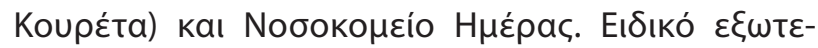

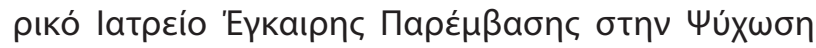

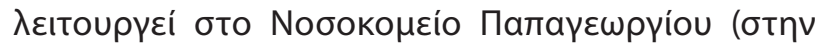

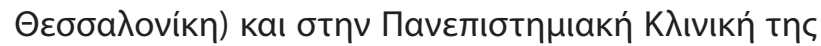
Пátpac.

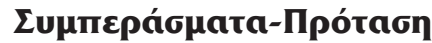

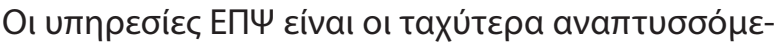

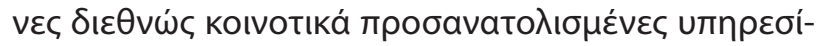

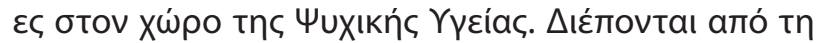

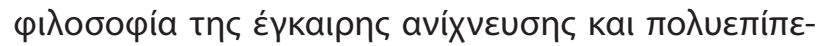

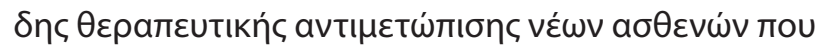

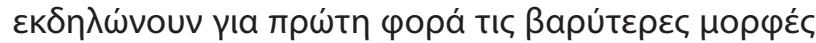

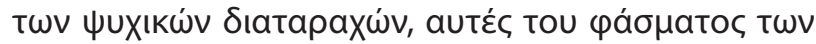

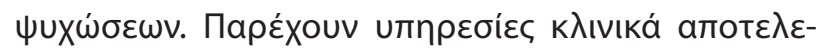

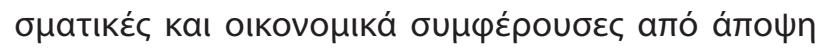

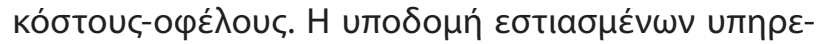

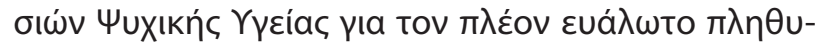

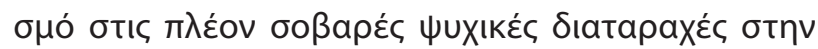

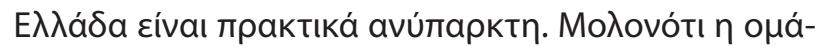

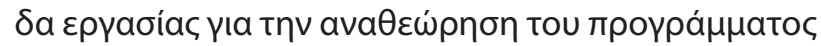

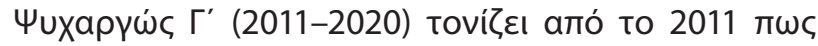

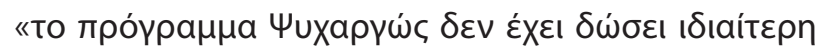

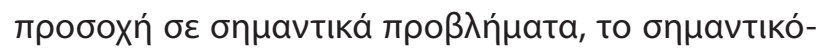

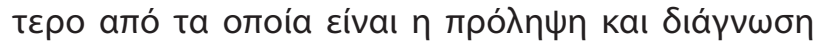

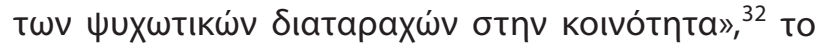

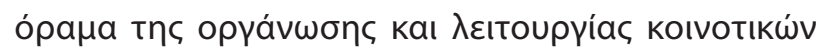

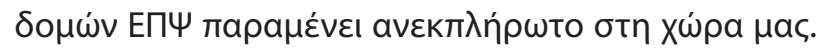

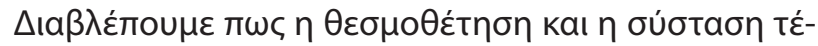

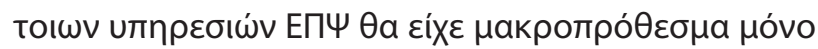

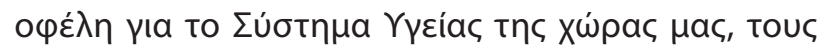

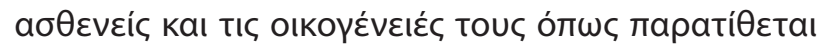

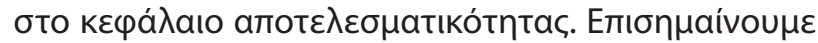

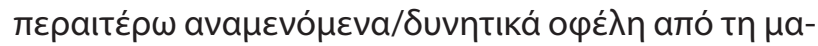

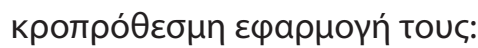

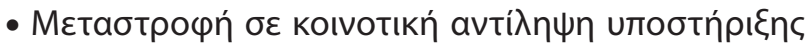

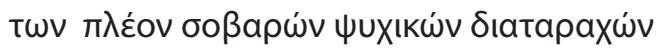

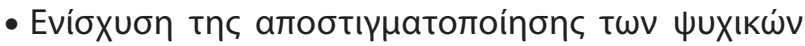
Siatapaxúv

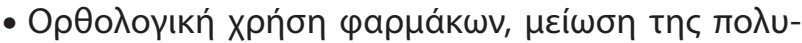
чарнакі́as

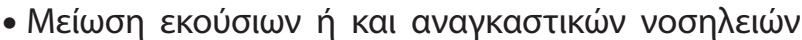

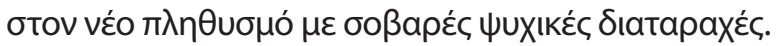




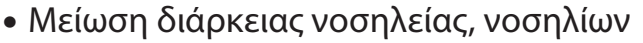

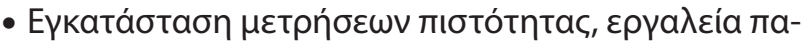

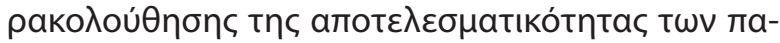

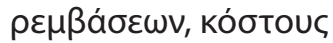

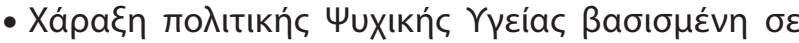

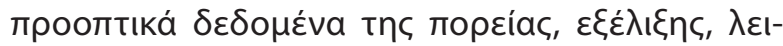

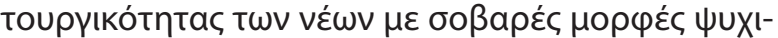

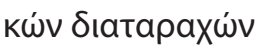

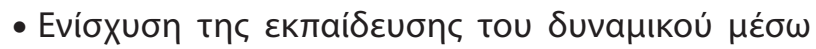

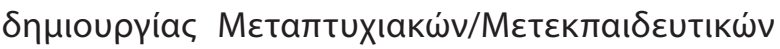

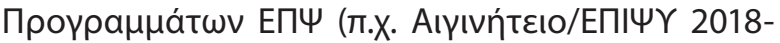
2019).

\title{
A proposal for the implementation of Early Intervention in Psychosis (EIP) services in Greece: If not now, when?
}

\author{
N.C. Stefanis, ${ }^{1}$ V. Mavreas, ${ }^{2}$ I. Nimatoudis, ${ }^{3}$ F. Gourzis, ${ }^{4}$ \\ M. Sarakouri, ${ }^{5}$ A. Vgontzas, ${ }^{6}$ A. Douzenis, ${ }^{7}$ EIP Working group ${ }^{8}$ \\ ${ }^{1} 1$ st Department of Psychiatry, National and Kapodistrian University of Athens, Athens, \\ ${ }^{2}$ Department of Psychiatry, University of loannina, loannina, \\ ${ }^{3} 3 r d$ Department of Psychiatry, Aristotelion University of Thessaloniki, Thessaloniki, \\ ${ }^{4}$ Department of Psychiatry, University General Hospital of Patra, Patra, \\ ${ }^{5}$ Department of Psychiatry, Dimokrition University of Thrace, Alexandroupolis, \\ ${ }^{6}$ Section of Psychiatry and Behavioral Sciences, University of Crete, Heraklion, Crete, \\ '2nd Department of Psychiatry, National and Kapodistrian University of Athens, Athens, Greece \\ ${ }^{8}$ V. Bozikas, K. Kollias, G. Garyfallos, M. Malliori, V. Ermiliou, P. Petrikis, M. Rizos, M. Economou
}

Psychiatriki 2018, 29:107-117

The disorders of the psychosis spectrum, with the most severe being schizophrenia, are prevalent and have a great impact on the patients' quality of life. The purpose of this article is to highlight the need for a novel national strategic approach to the management of psychotic disorders in accordance with the international principles of early intervention. Even though outpatient treatment is considered adequate, there is an urgent need to adopt an early and more comprehensive and effective intervention strategy for young patients with psychosis and their families whose clinical and personal needs are clearly not met by the existing infrastructure of our mental health services. This can be accomplished by the legislation and implementation within the national health system of EIP services which on the one hand actively engage community organizations with the purpose of early identification of cases, reduction of the duration of untreated psychosis and on the other, offer assertive community-based support and treatment, based on a multi-disciplinary community team model. The effectiveness of early intervention in psychosis is supported by evidence provided by 9 international RCTs. The results of these programs indicate a superior effect in indexes of quality of life, retention in treatment, psychopathology, judicious use of medication and return to work/school as well as the patient's effective recovery. International experience (Denmark, Norway, Australia, UK, USA, Canada and Italy) and the corresponding prevention programs emphasize the effectiveness of 
EIP services and thus the patients' reintegration. However, in contrast to Northern European countries, Southern European countries have not yet incorporated EIP services in their national health system. From a financial perspective, EIP services seem to be cost-effective for the national health system, since the economic burden is compensated in the long term through their qualitative benefits. In Greece, specialized services for those young afflicted for the first time by the most serious of mental disorders are non-existent and no local information exists for the patient's outcome and social integration after a first psychotic-episode nor for the financial burden, placed on mental health services. Overall, the implementation of EIP services is expected to have long-term benefits for our country's National Health System as well as for the patients and their families.

Key words: Early detection, integrated intervention, first episode psychosis, preventive psychiatry.

\section{BıBลtoypacpía}

1. Perälä J, Suvisaari J, Saarni SI, Kuoppasalmi K, Isometsä E, Pirkola $S$ et al. Lifetime prevalence of psychotic and bipolar I disorders in a general population. Arch Gen Psychiatry 2007, 64:19-28, doi:10.1001/archpsyc.64.1.19

2. Jongsma HE, Gayer-Anderson C, Lasalvia A, Quattrone D, Mulè A, Szöke A, et al. Treated incidence of psychotic disorders in the multinational EU-GEI study. JAMA Psychiatry 2018, 75:3646, doi:10.1001/jamapsychiatry.2017.3554

3. Peritogiannis V, Mantas C, Tatsioni A, Mavreas V. Rates of first episode of psychosis in a defined catchment area in Greece. Clinical practice and epidemiology in mental health: CP \& EMH 2013, 9:251, doi: $10.2174 / 1745017901309010251$

4. Vos T, Barber RM, Bell B, Bertozzi-Villa A, Biryukov S, Bolliger I,et al. Global, regional, and national incidence, prevalence, and years lived with disability for 301 acute and chronic diseases and injuries in 188 countries, 1990-2013: a systematic analysis for the Global Burden of Disease Study 2013. Lancet 2015, 386:743-800, doi:10.1016/S0140-6736(15)60692-4

5. Murray CJ, Lopez AD. Alternative projections of mortality and disability by cause 1990-2020: Global Burden of Disease Study. Lancet 1997, 349:1498-504, doi: 10.1016/S01406736(96)07492-2

6. Alvarez-Jimenez M, Priede A, Hetrick SE, Bendall S, Killackey E, Parker AG et al. Risk factors for relapse following treatment for first episode psychosis: a systematic review and meta-analysis of longitudinal studies. Schizophr Res 2012, 139:116-28, doi:/10.1016/j.schres.2012.05.007

7. Penttilä $M$, Jääskeläinen $E$, Hirvonen $N$, Isohanni M, Miettunen J. Duration of untreated psychosis as predictor of long-term outcome in schizophrenia: systematic review and meta-analysis. $\mathrm{Br}$ J Psychiatry 2014, 205:88-94, doi:10.1192/bjp.bp.113.127753

8. Fusar-Poli P, McGorry PD, Kane JM. Improving outcomes of first-episode psychosis: an overview. World Psychiatry 2017, 16:251-65, doi:/10.1002/wps.20446

9. Craig TK, Garety P, Power P, Rahaman N, Colbert S, FornellsAmbrojo M, Dunn G. The Lambeth Early Onset (LEO) Team: randomised controlled trial of the effectiveness of specialised care for early psychosis. BMJ 2004, 329:1067, doi: 10.1136/ bmj.38246.594873.7C
10. Petersen L, Jeppesen $P$, Thorup A, Abel MB, ØhlenschlZger $\mathrm{J}$, Christensen TØ et al. A randomised multicentre trial of integrated versus standard treatment for patients with a first episode of psychotic illness. BMJ 2005, 331:602, doi: 10.1136/ bmj.38565.415000.E01

11. Kane JM, Robinson DG, Schooler NR, Mueser KT, Penn DL, Rosenheck RA et al. Comprehensive versus usual community care for first-episode psychosis: 2-year outcomes from the NIMH RAISE early treatment program. Am J Psychiatry 2015, 173:362-72, doi: 10.1176/appi.ajp.2015.15050632

12. Grawe RW, Falloon IR, Widen JH, Skogvoll E. Two years of continued early treatment for recent-onset schizophrenia: a randomised controlled study. Acta Psychiatr Scand 2006, 114:328-36, doi: 10.1111/j.1600-0447.2006.00799.x

13. Srihari VH, Tek C, Kucukgoncu S, Phutane VH, Breitborde $\mathrm{NJ}$, Pollard $\mathrm{J}$ et al. First-episode services for psychotic disorders in the US public sector: a pragmatic randomized controlled trial. Psychiatr Serv 2015, 66:705-712, doi: 10.1176/ appi.ps.201400236

14. Ruggeri M, Bonetto C, Lasalvia A, Fioritti A, De Girolamo G, Santonastaso $P$, et al. Feasibility and effectiveness of a multielement psychosocial intervention for first-episode psychosis: results from the cluster-randomized controlled GET UP PIANO trial in a catchment area of 10 million inhabitants. Schizophr Bull 2015, 41:1192-1203, doi: 10.1093/schbul/sbv058

15. Nordentoft $M$, Melau $M$, Iversen $T$, Petersen $L$, Jeppesen $P$, Thorup A, et al. From research to practice: how OPUS treatment was accepted and implemented throughout Denmark. Early Interv Psychiatry 2015, 9:156-162, doi: 10.1111/eip.12108

16. Albert N, Melau M, Jensen H, Emborg C, Jepsen JR, Fagerlund $B$, et al. Five years of specialised early intervention versus two years of specialised early intervention followed by three years of standard treatment for patients with a first episode psychosis: randomised, superiority, parallel group trial in Denmark (OPUS II). BMJ 2017, 356:i6681, doi: 10.1136/bmj.i6681

17. Csillag C, Nordentoft M, Mizuno M, McDaid D, Arango C, Smith $\mathrm{J}$, et al. Early intervention in psychosis: From clinical intervention to health system implementation. Early Interv Psychiatry 2017, doi: 10.1111/eip. 12514 
18. Melle I, Larsen TK, Haahr U, Friis S, Johannesen JO, Opjordsmoen $S$ et al. Prevention of negative symptom psychopathologies in first-episode schizophrenia: two-year effects of reducing the duration of untreated psychosis. Arch Gen Psychiatry 2008, 65:634-640, doi: 10.1001/archpsyc.65.6.634

19. Hegelstad WT, Larsen TK, Auestad B, Evensen J, Haahr U, Joa I et al. Long-term follow-up of the TIPS early detection in psychosis study: effects on 10-year outcome. Am J Psychiatry 2012, 169:374-80, doi: 10.1176/appi.ajp.2011.11030459

20. Valmaggia LR, McCrone P, Knapp M, Woolley JB, Broome MR, Tabraham $\mathrm{P}$, et al. Economic impact of early intervention in people at high risk of psychosis. Psychol Med 2009, 39:1617-1626, doi: 10.1017/S0033291709005613

21. Angelo C, Vittorio M, Anna M, Antonio P. Cost-effectiveness of treating first-episode psychosis: five-year follow-up results from an Italian early intervention programme. Early Interv Psychiatry 2011, 5:203-211, doi: 10.1111/j.1751-7893.2011.00261.x

22. Bertolote J, McGorry P. Early intervention and recovery for young people with early psychosis: consensus statement. $\mathrm{Br} \mathrm{J}$ Psychiatry 2005, 187:s116-119, doi: 10.1192/bjp.187.48.s116

23. McCrone P, Knapp M, Dhanasiri S. Economic impact of services for first-episode psychosis: a decision model approach. Early Interv Psychiatry 2009, 3:266-273, doi: 10.1111/j.17517893.2009.00145.x

24. McCrone P, Craig TK, Power P, Garety PA. Cost-effectiveness of an early intervention service for people with psychosis. Br J Psychiatry 2010, 196:377-382, doi: 10.1192/bjp.bp.109.065896

25. Tsiachristas A, Thomas T, Leal J, Lennox BR. Economic impact of early intervention in psychosis services: results from a longitudinal retrospective controlled study in England. BMJ 2016, 6:e012611, doi: 10.1136/bmjopen-2016-012611

26. Park AL, McCrone $P$, Knapp M. Early intervention for firstepisode psychosis: broadening the scope of economic estimates. Early Interv Psychiatry 2016, 10:144-151, doi: 10.1111/ eip. 12149

27. Hastrup LH, Kronborg C, Bertelsen M, Jeppesen P, Jorgensen $P$, Petersen $L$ et al. Cost-effectiveness of early intervention in first-episode psychosis: economic evaluation of a randomised controlled trial (the OPUS study). Br J Psychiatry 2013, 202:35-41, doi: 10.1192/bjp.bp.112.112300

28. Mihalopoulos C, Harris M, Henry L, Harrigan S, McGorry P. Is early intervention in psychosis cost-effective over the long term? Schizophr Bull 2009, 35:909-918, doi: 10.1093/schbul/ sbp054

29. Rosenheck R, Leslie D, Sint K, Lin H, Robinson DG, Schooler $\mathrm{NR}$, et al. Cost-effectiveness of comprehensive, integrated care for first episode psychosis in the NIMH RAISE Early Treatment Program. Schizophr Bull 2016, 42:896-906, doi: 10.1093/schbul/sbv224

30. Mantas C, Mavreas V. Establishing and operating an early intervention service for psychosis in a defined catchment area of northwestern Greece within the context of the local mental health network. Early Interv Psychiatry 2012, 6:212-217, doi: 10.1111/j.1751-7893.2012.00358.x

31. Kollias C, Xenaki LA, Dimitrakopoulos S, Kosteletos I, Kontaxakis V, Stefanis N, Papageorgiou C. Early psychosis intervention outpatient service of the 1st Psychiatric University Clinic in Athens: 3 Years of experience. Early Interv Psychiatry 2016, doi: 10.1111/eip.12407

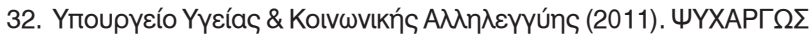

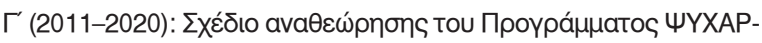
$\Gamma \Omega \Sigma$. Available at: www.psychargos.gov.gr/Documents2/NEA/ ЧYХАРГ $\Omega$ $\Sigma 20 Г \% 20(2011-2020)$ (Last accessed: 23/04/2018)

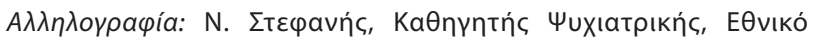

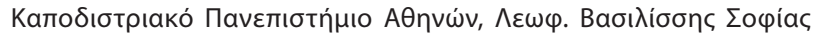
72-74, 11528 AӨńva, E $\lambda \lambda a ́ \delta a$ e-mail: nistefan@med.uoa.gr, nikos41161@gmail.com 


\title{
Eıठı́ó ápӨpo Special article
}

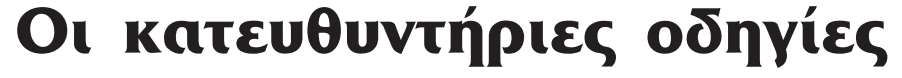

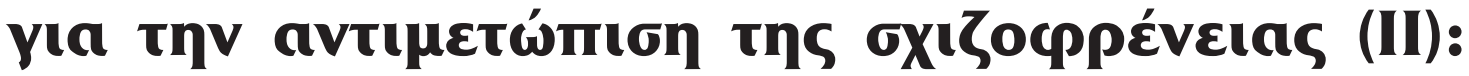

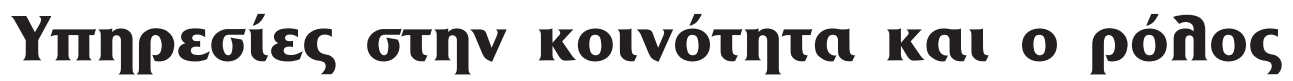

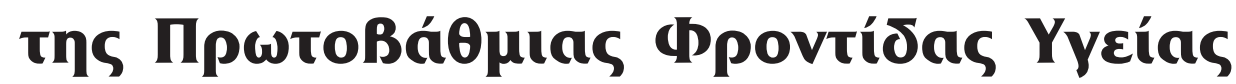

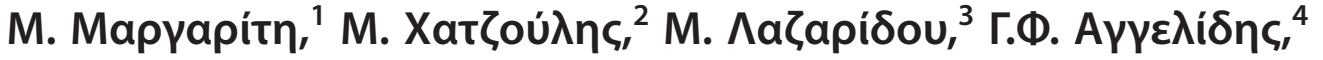

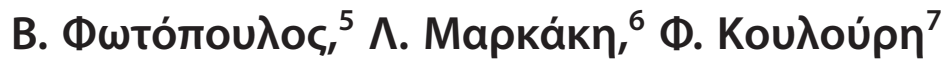

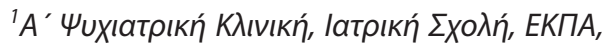

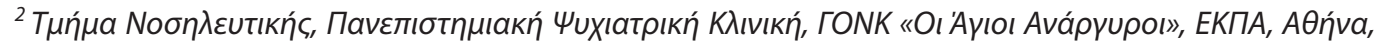

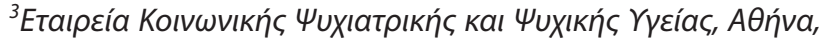

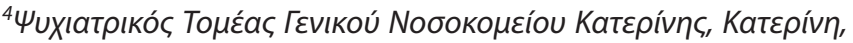

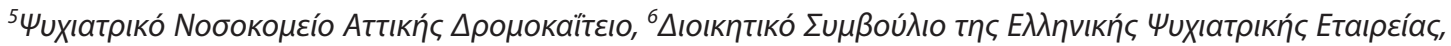

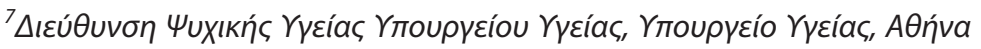

Uuxıatpıkń 2018, 29:118-129

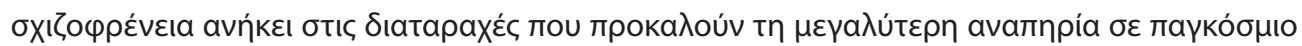

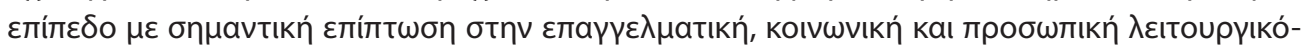

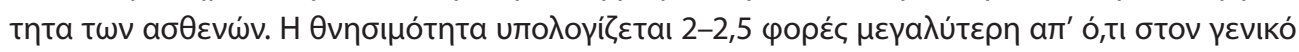

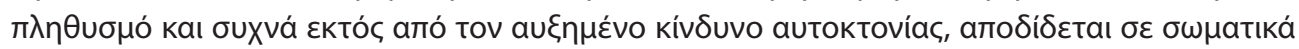

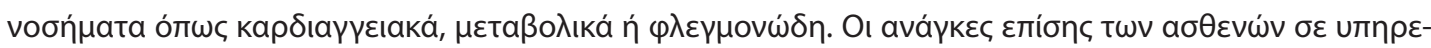

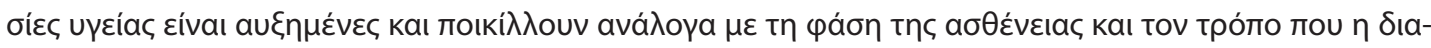

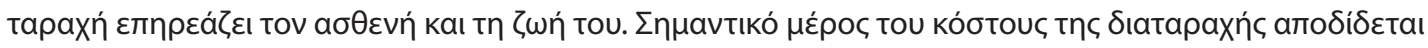

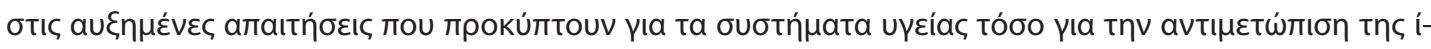

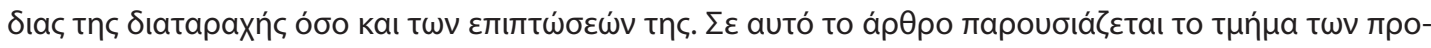

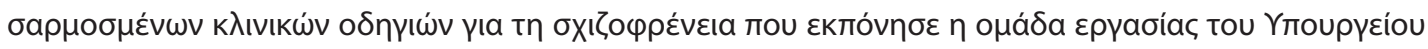

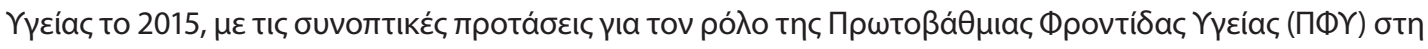

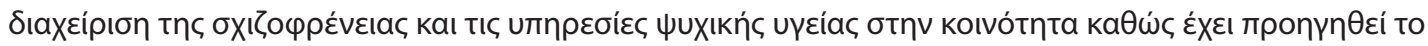

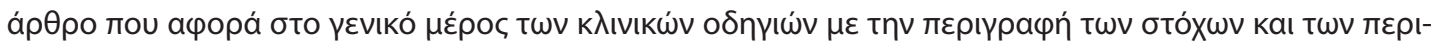

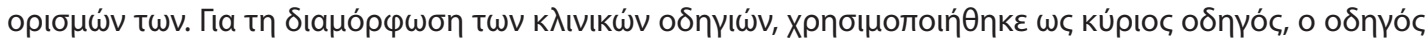

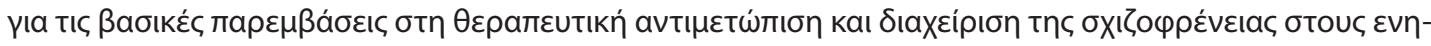




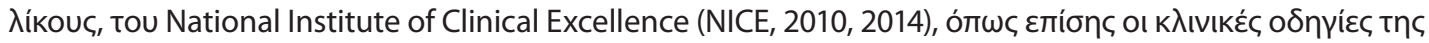

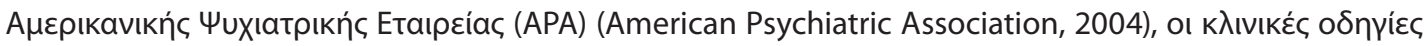
tou Royal Australian and New Zealand College of Psychiatrists (2005), kaӨ

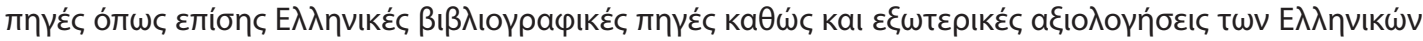

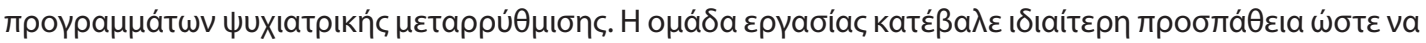

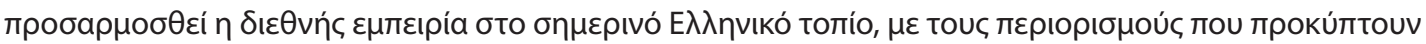

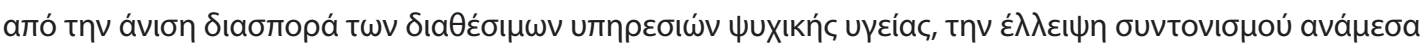

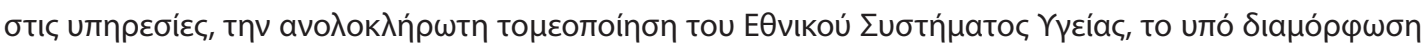

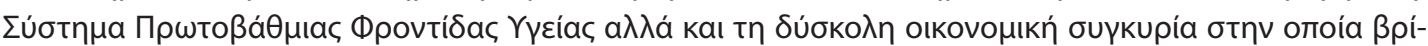

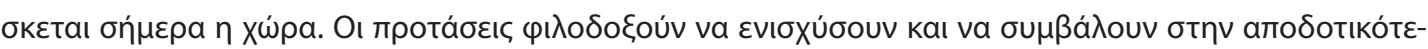

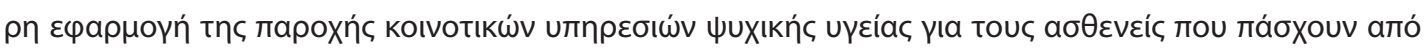

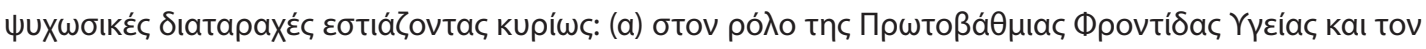

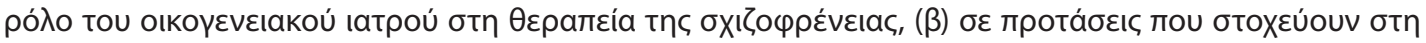

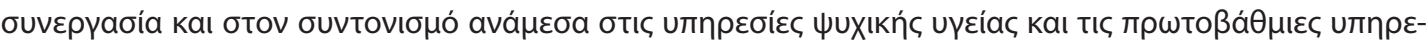

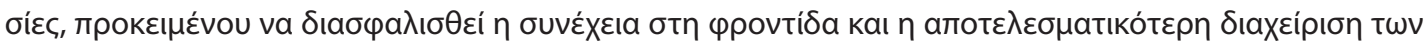

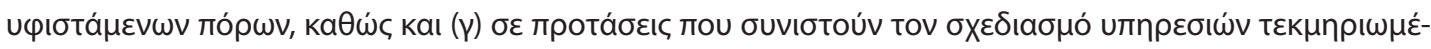

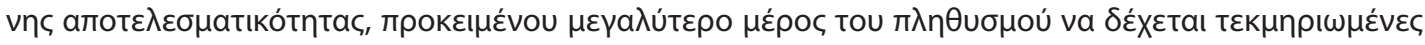

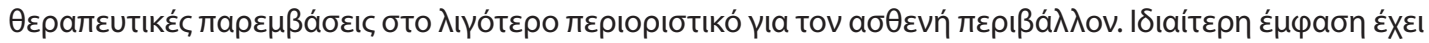

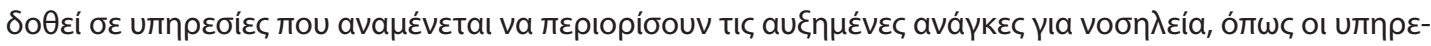

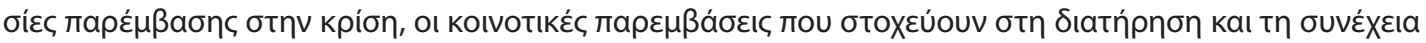

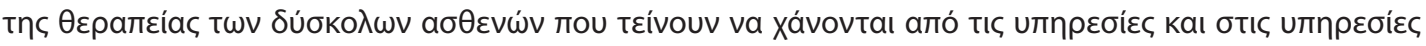

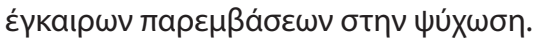

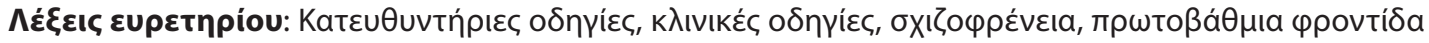

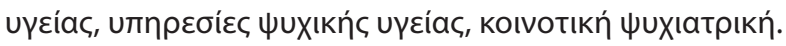

\section{Eıarywŕn}

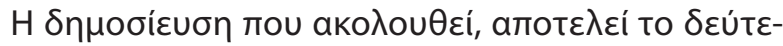

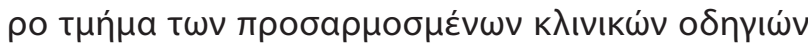

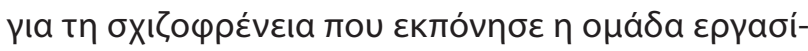

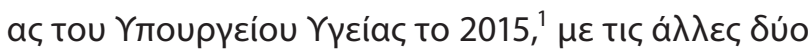

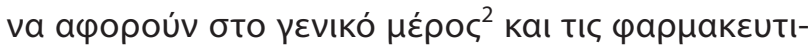

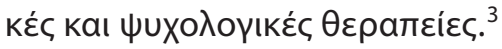

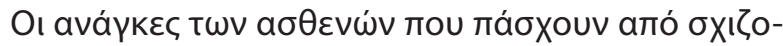

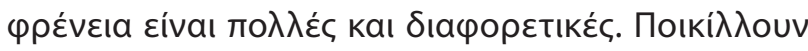

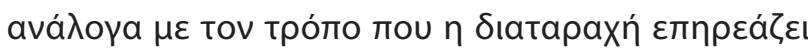

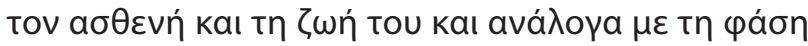

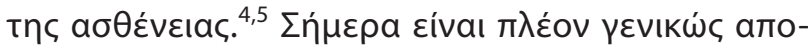

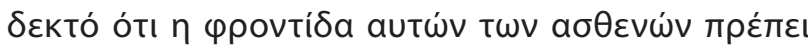

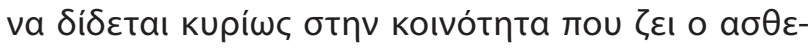

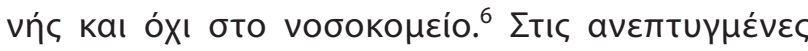

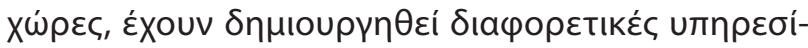

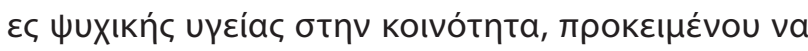

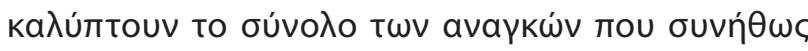

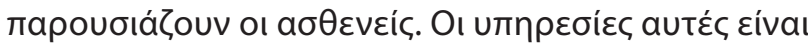

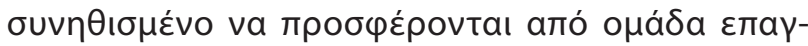

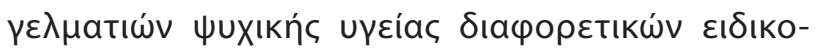

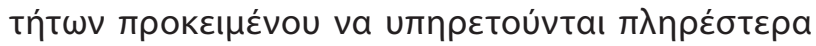

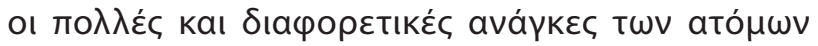

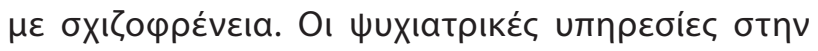

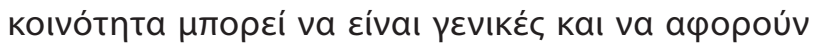

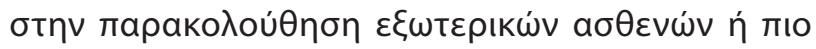

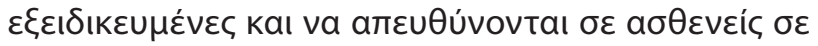

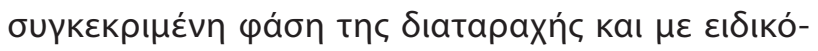

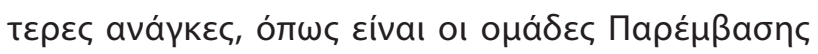

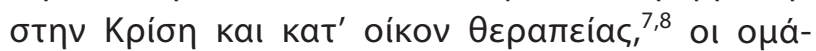

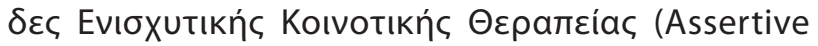

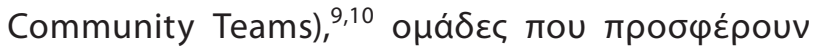

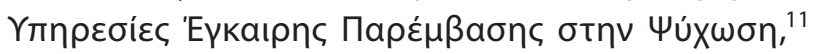

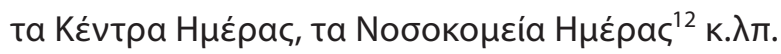

$\Sigma \tau \eta$ X

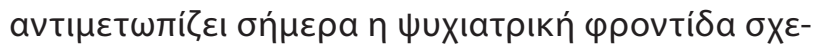

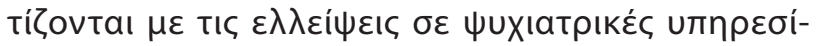

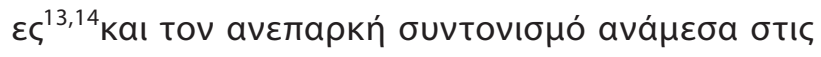

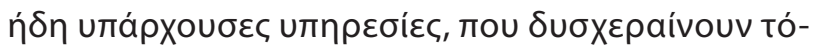

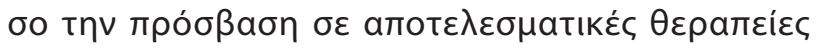

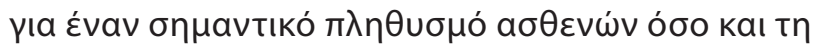




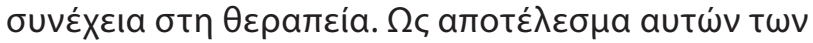

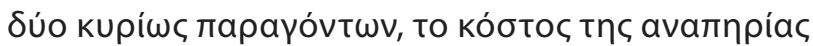

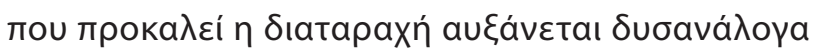

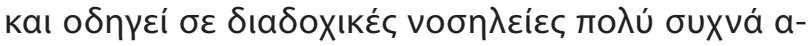

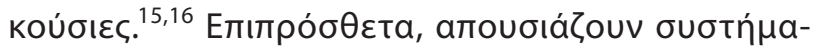

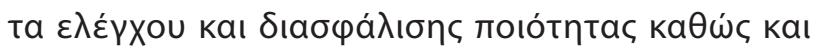

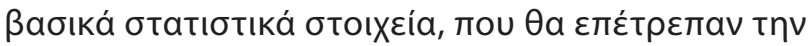

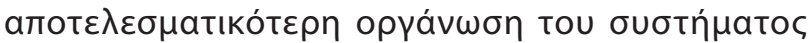

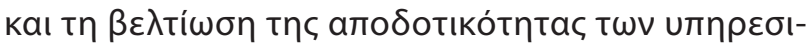
$\omega \dot{v} .^{17,18}$

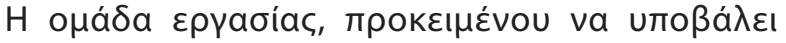

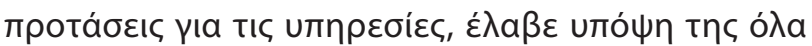

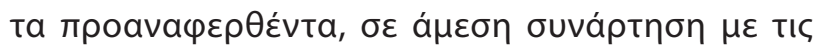

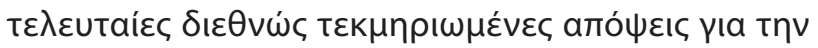

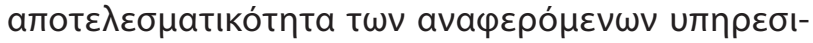

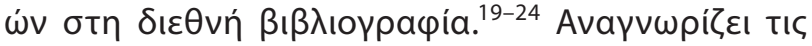

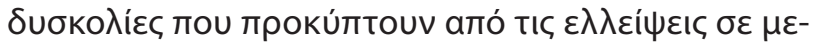

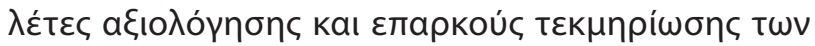

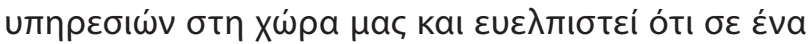

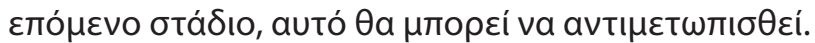
КaӨ

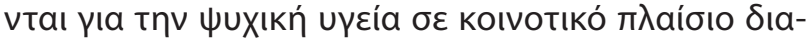

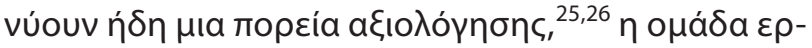

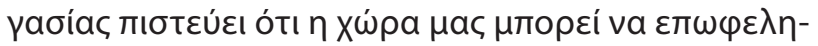

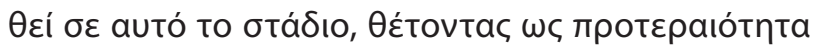

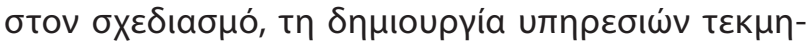

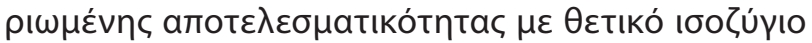

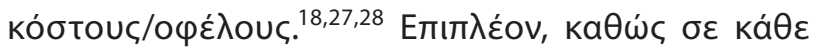

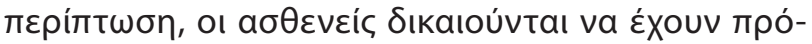

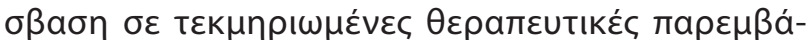

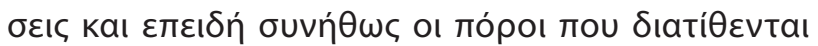

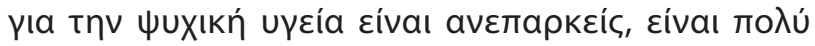

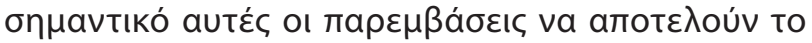

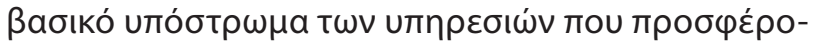

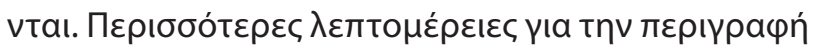

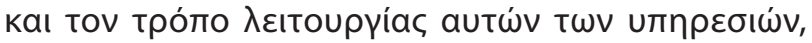

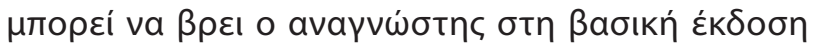

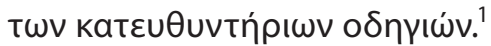

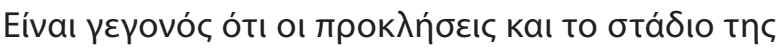

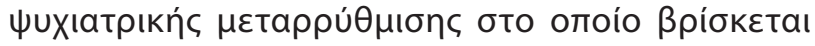

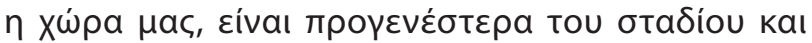

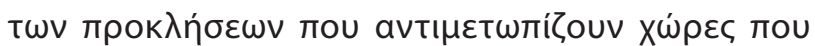

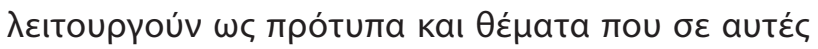

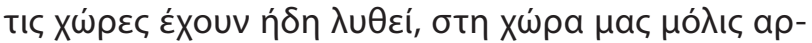

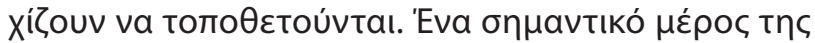

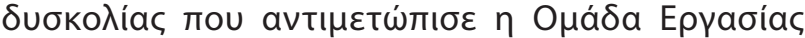

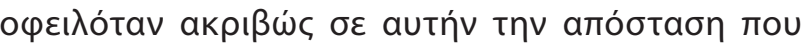

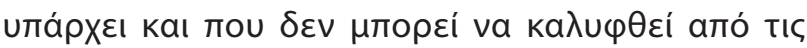

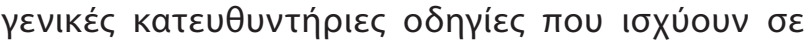

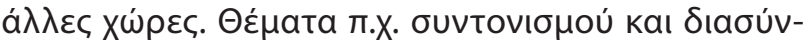

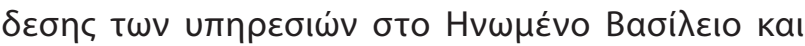

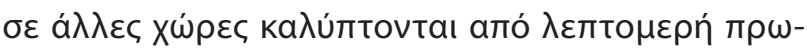

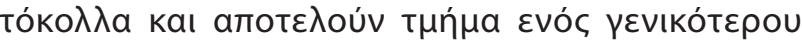

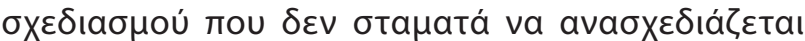

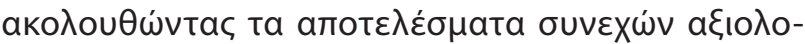

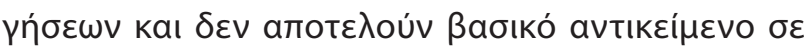

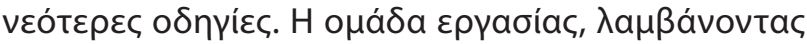

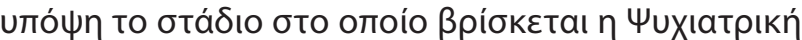

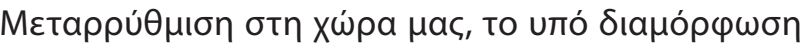

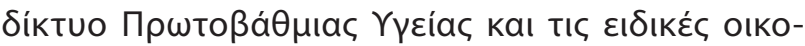

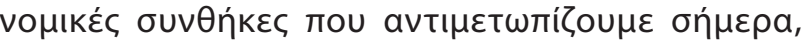

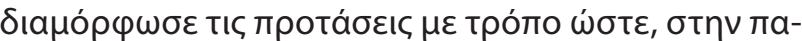

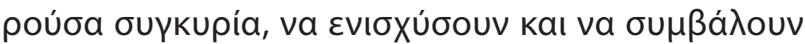

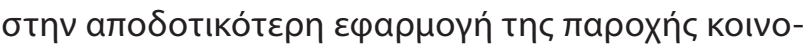

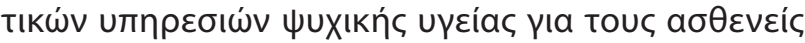

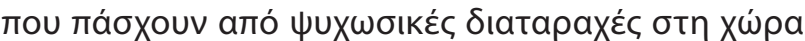

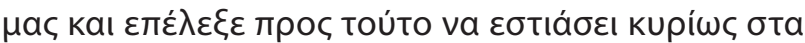

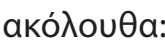

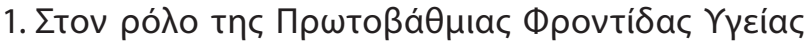

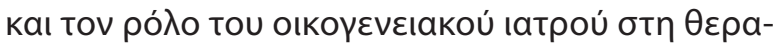

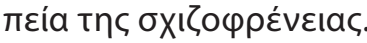

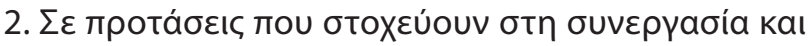

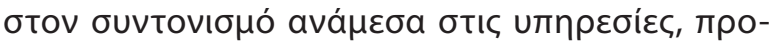

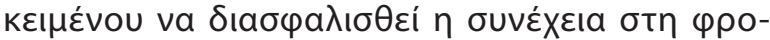

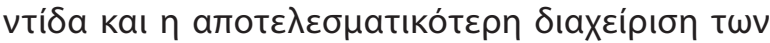

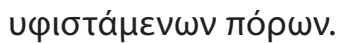

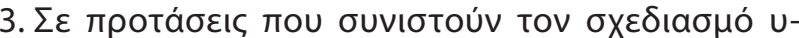

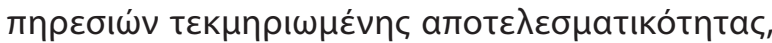

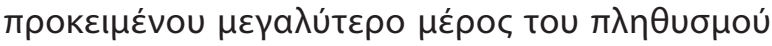

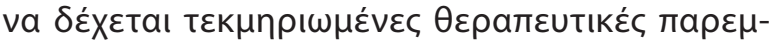

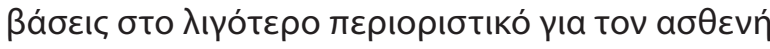

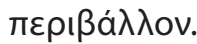

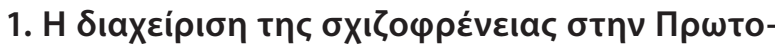

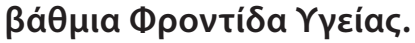

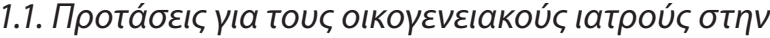

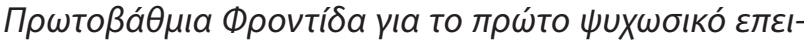

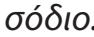

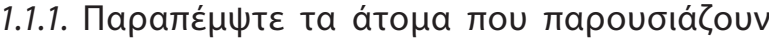

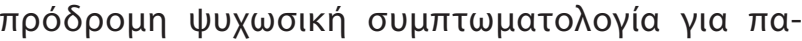




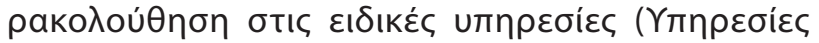

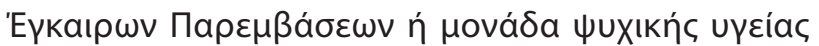

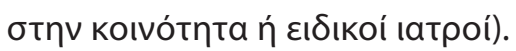

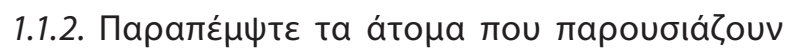

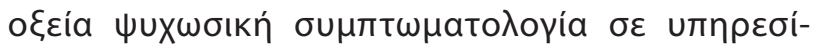

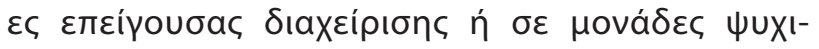

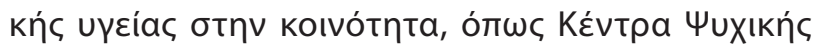

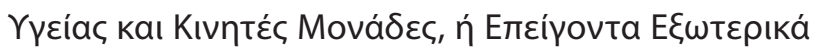

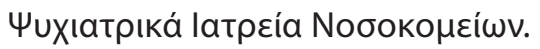

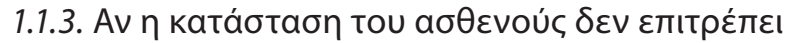

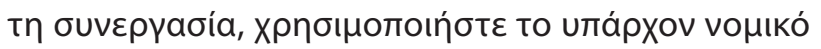

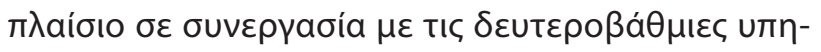

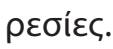

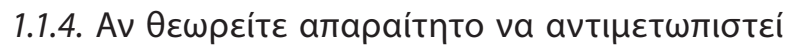

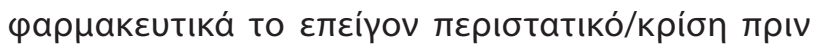

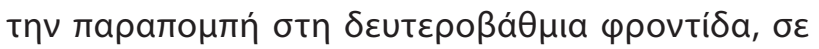

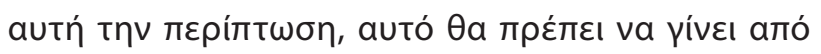

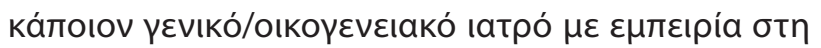

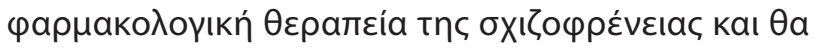

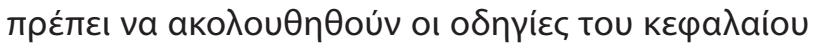

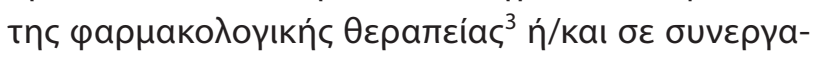

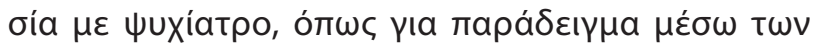

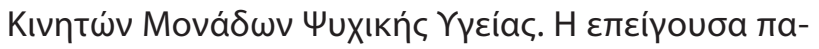

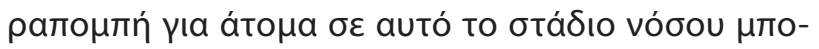

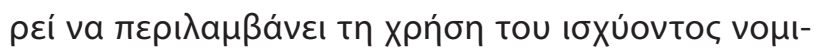

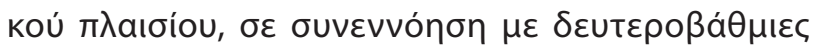

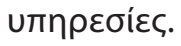

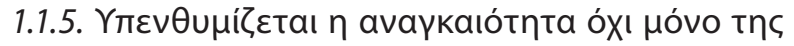

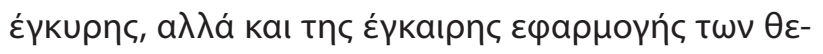

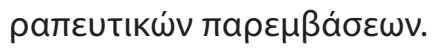

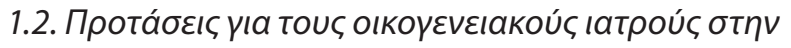

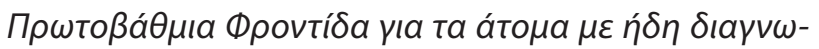

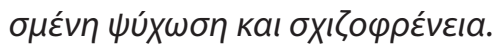

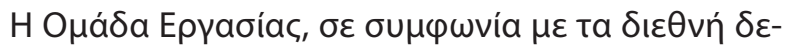

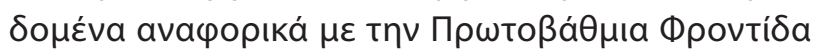

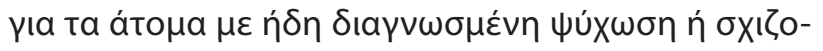

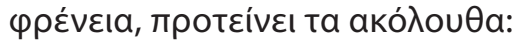

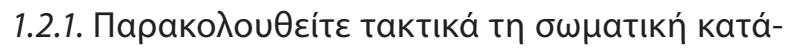

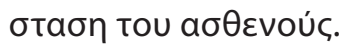

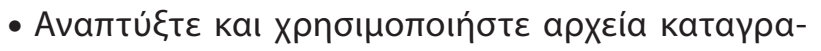

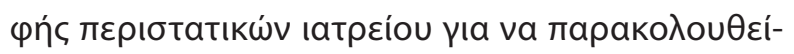

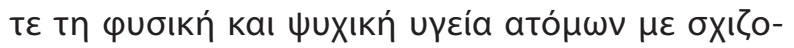

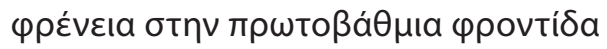

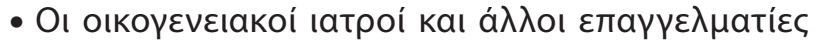

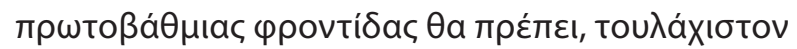

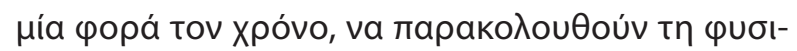

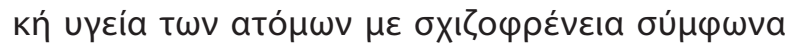

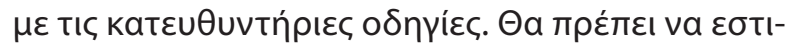

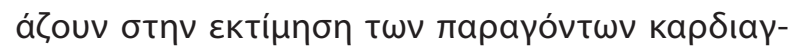

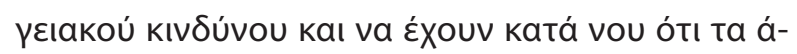

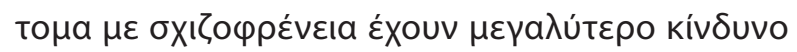

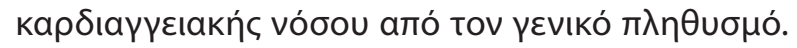

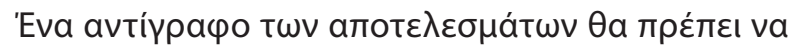

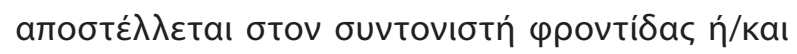

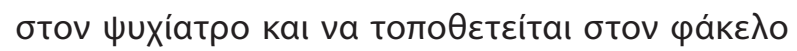

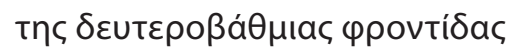

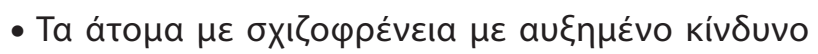

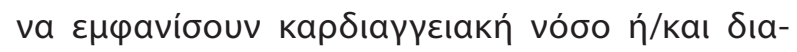

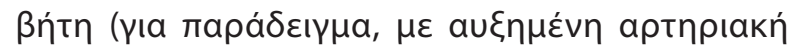

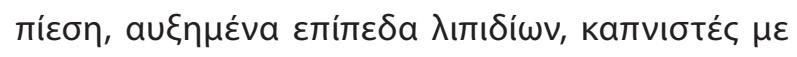

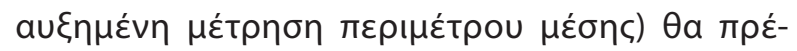

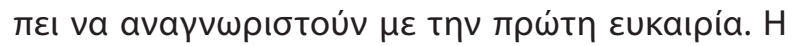

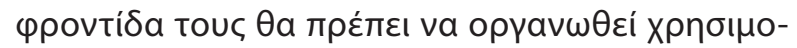

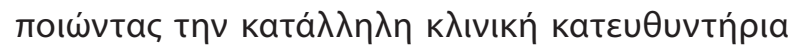

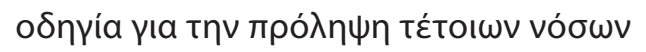

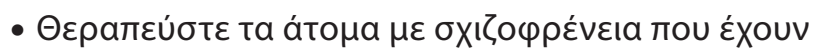

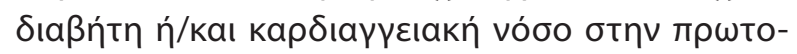

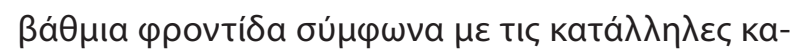

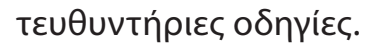

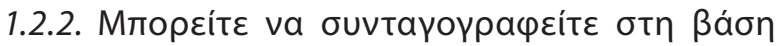

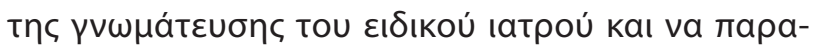

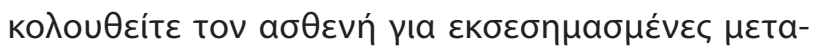

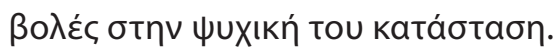

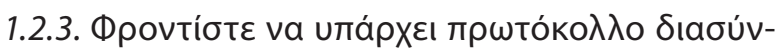

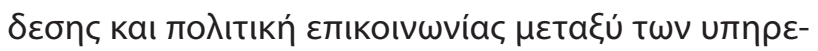

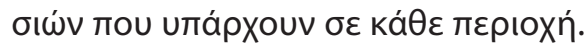

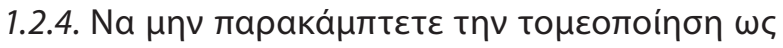

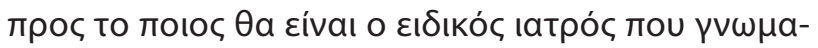

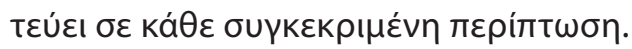

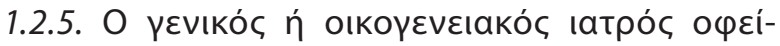

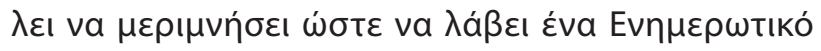

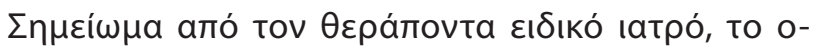

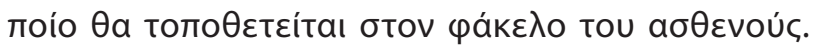

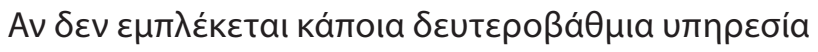

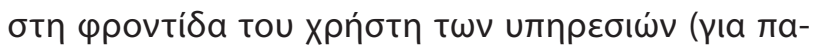

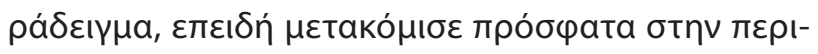

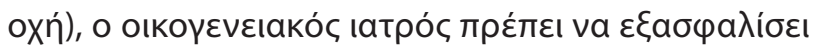

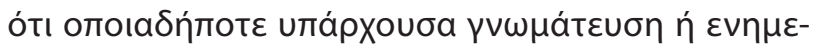




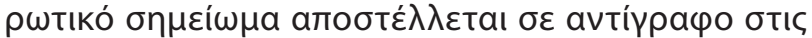

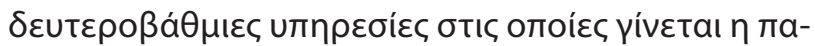
ратонти́.

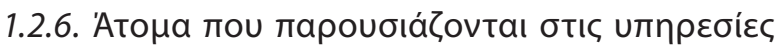

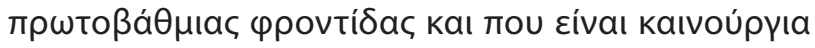

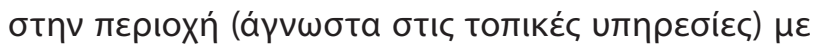

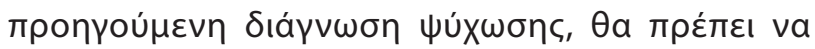

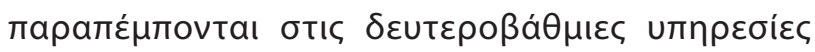

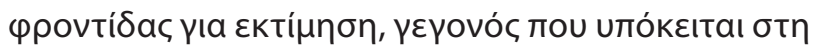

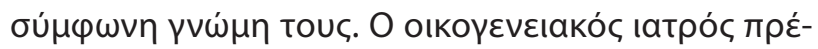

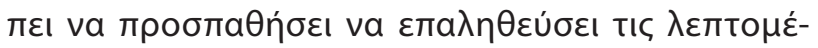

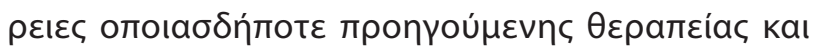

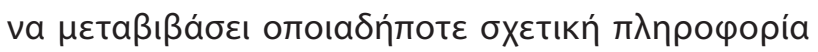

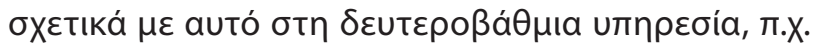

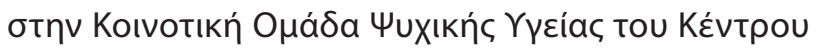

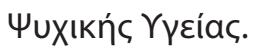

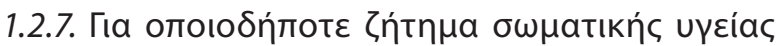

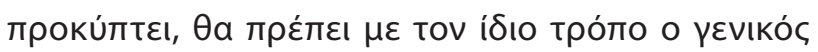

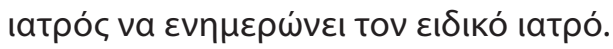

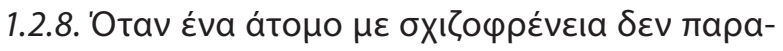

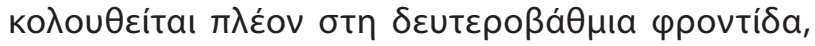

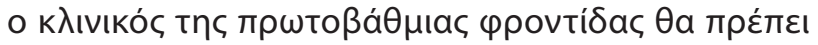

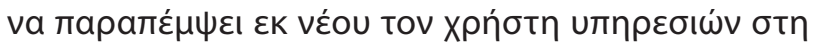

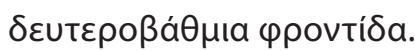

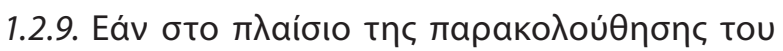

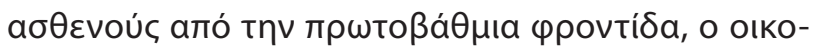

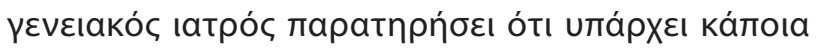

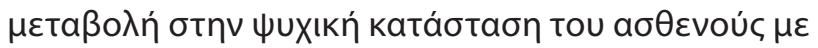

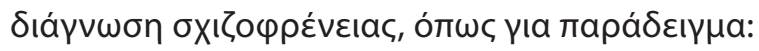

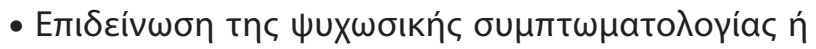

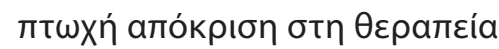

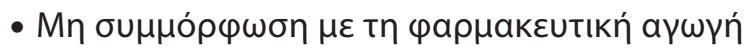

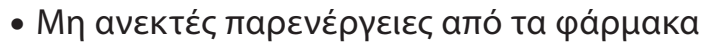

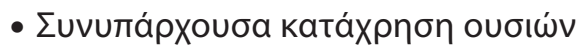

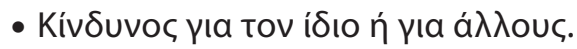

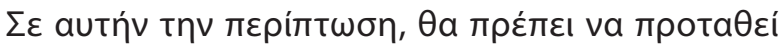

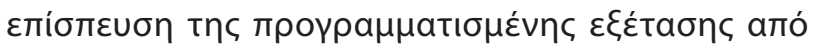

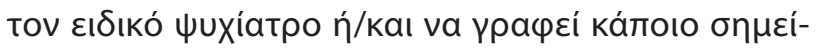

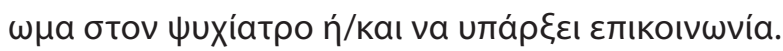

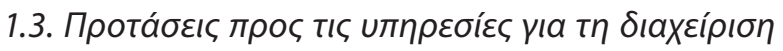

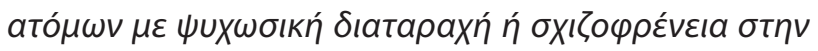

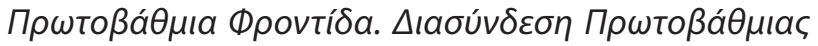

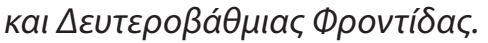

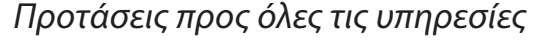

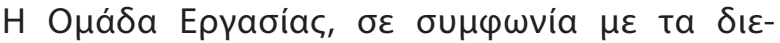

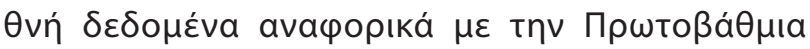

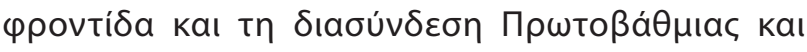

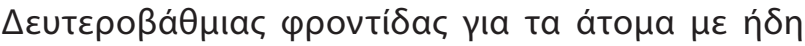

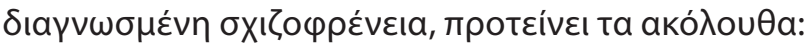

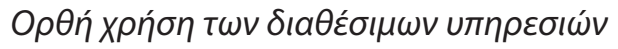

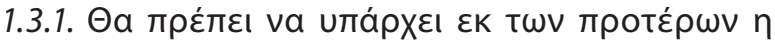

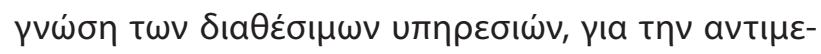

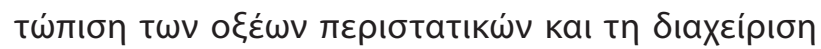

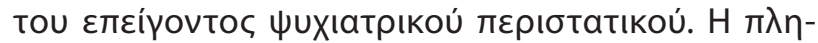

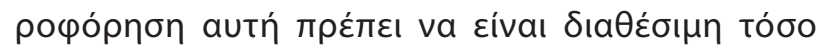

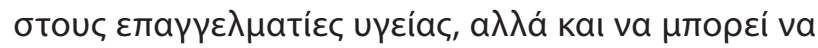

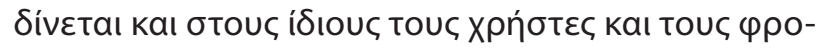

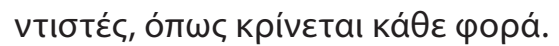

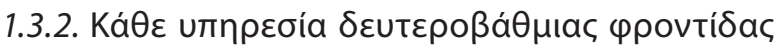

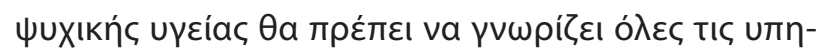

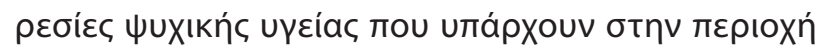

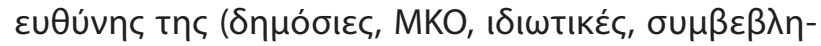

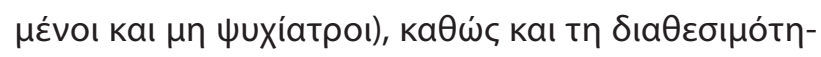

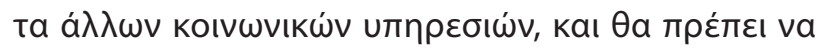

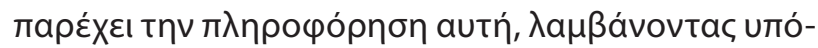

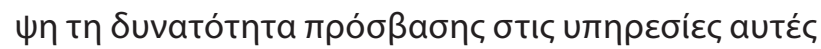

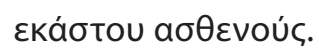

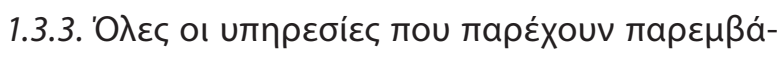

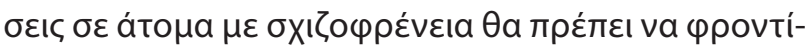

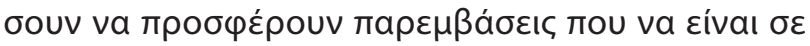

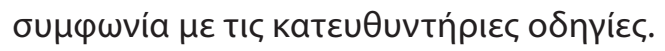

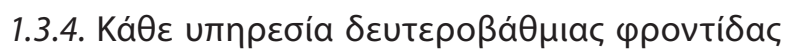

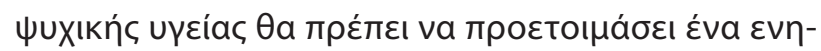
$\mu \varepsilon \rho \omega \tau$ тाкó $\varphi$

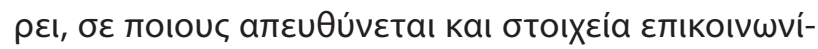

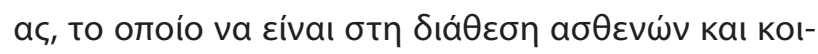

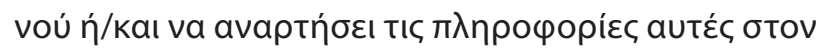

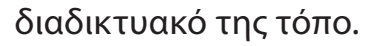

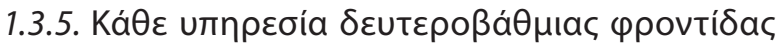

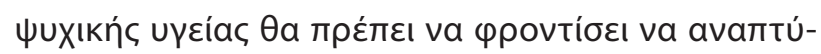

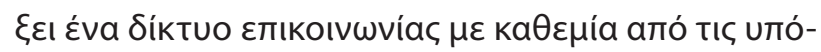

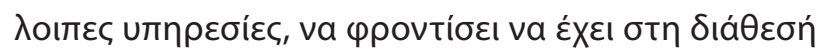

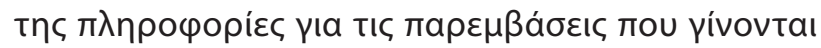

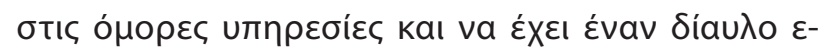

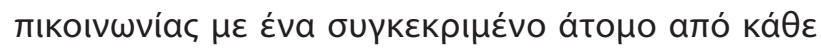
uтпребі́a. 


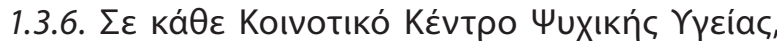

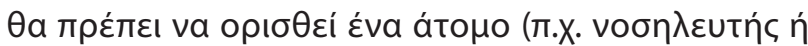

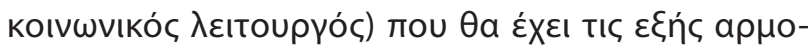

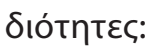

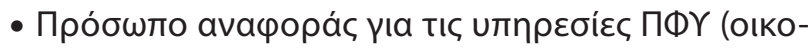

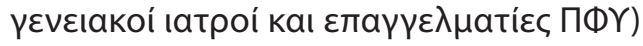

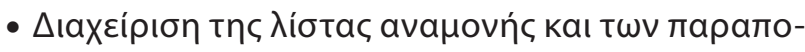

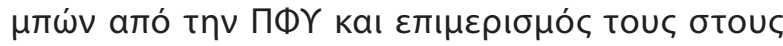

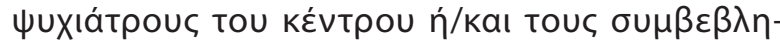

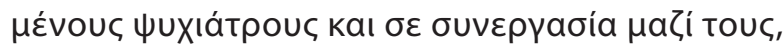

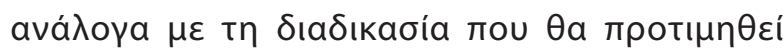

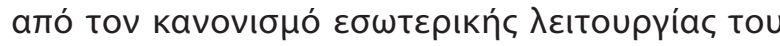
$\Pi \mathrm{E} \Delta \mathrm{Y}$.

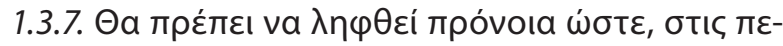

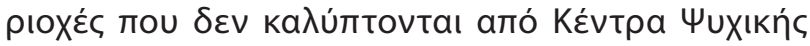

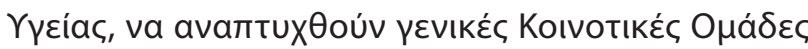

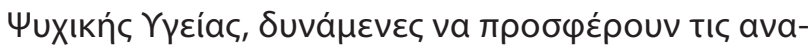

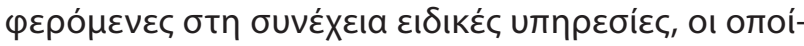

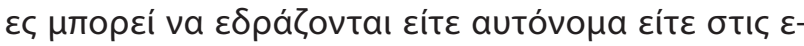

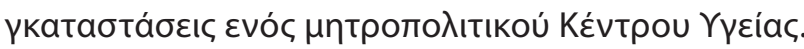

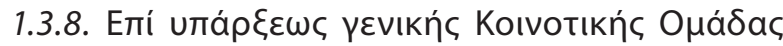

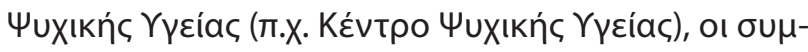

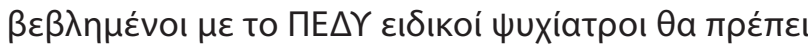

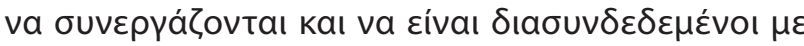

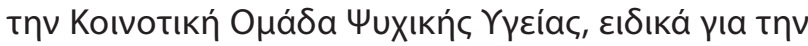

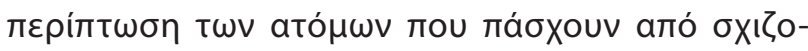
$\varphi \rho \varepsilon ́ v \varepsilon ı$.

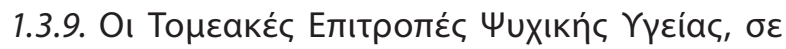

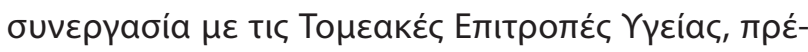
$\pi \varepsilon ı:$

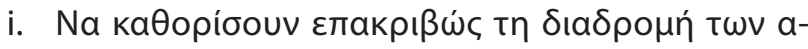

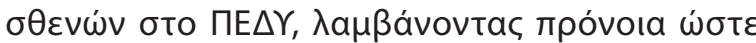

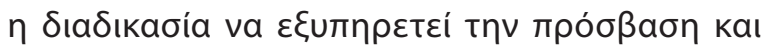

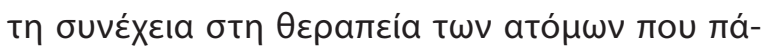

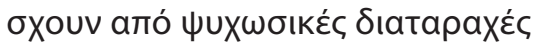

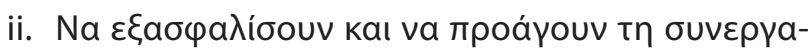

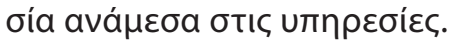

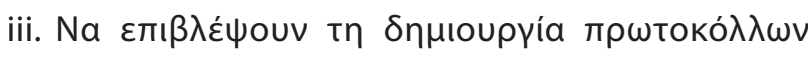

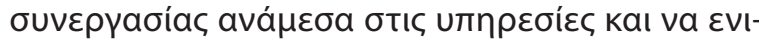

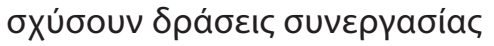

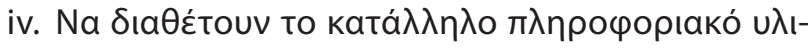

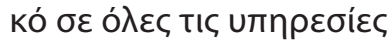

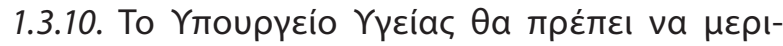

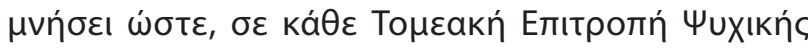

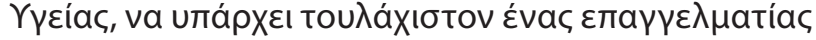

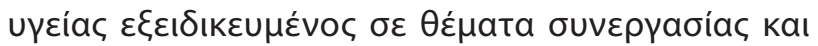

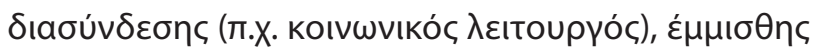

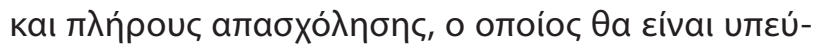

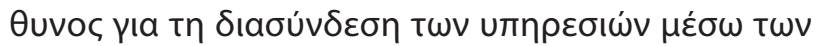

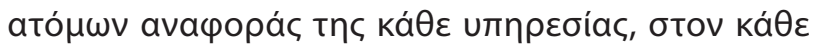

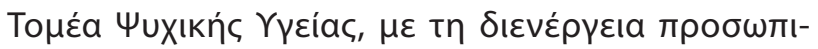

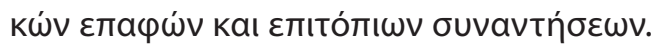

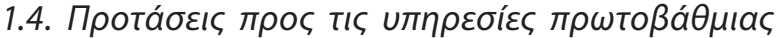

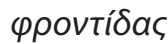

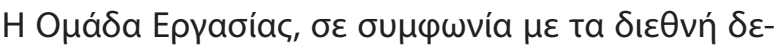

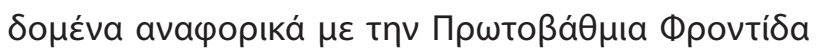

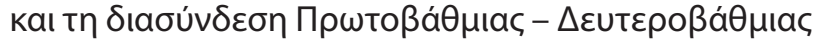

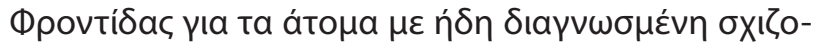

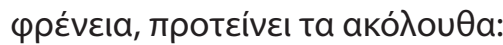

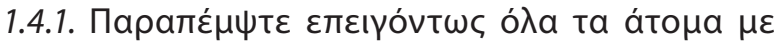

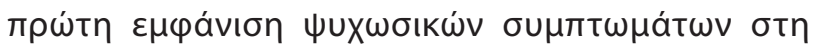

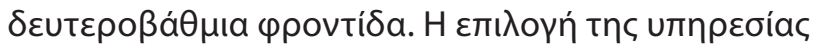

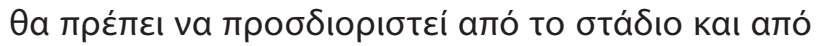

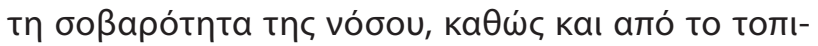

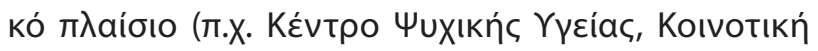

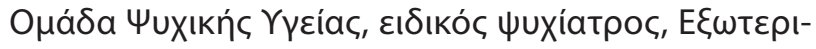

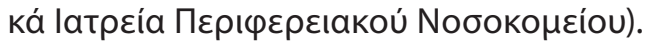

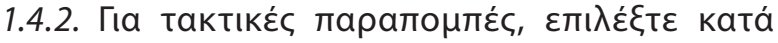

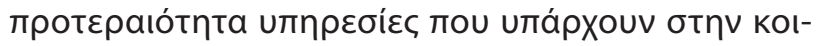

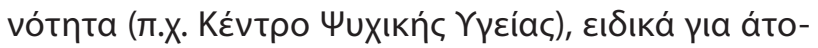

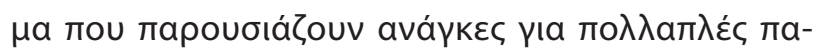

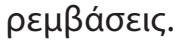

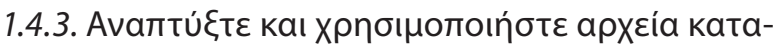

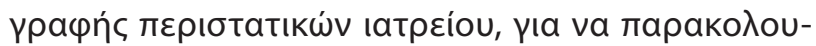

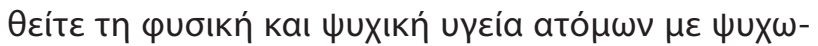

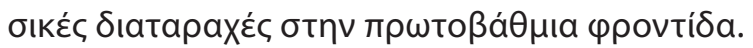

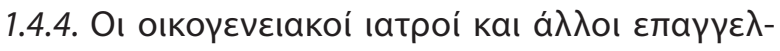

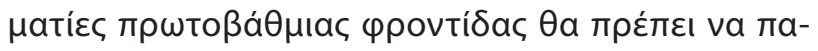

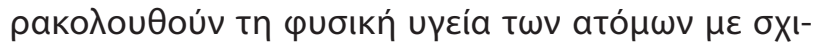

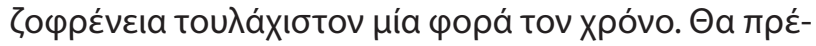

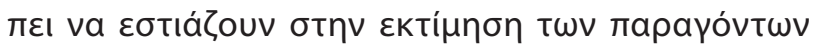

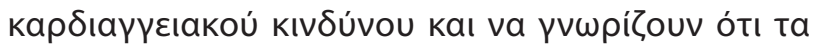
áto $\mu a \mu \varepsilon$ бXı

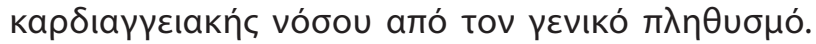

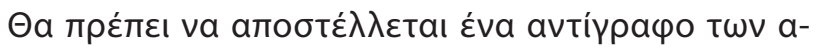

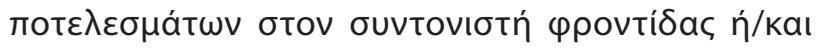

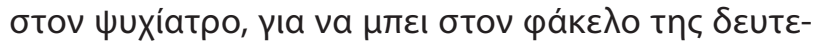

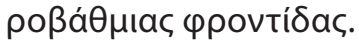




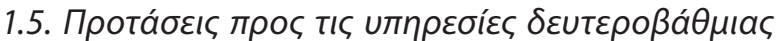

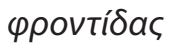

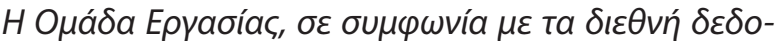

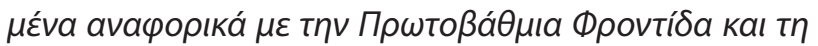

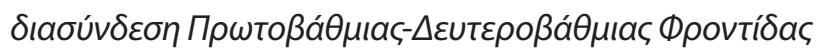

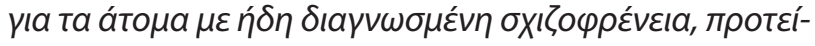
veı та aкódouӨa:

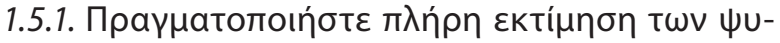

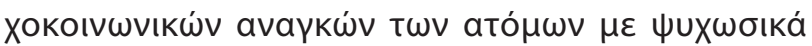

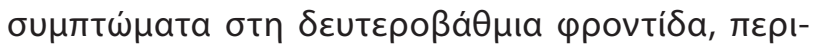

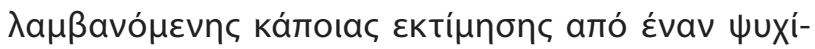

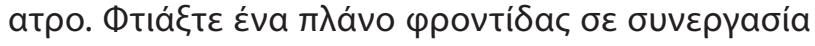

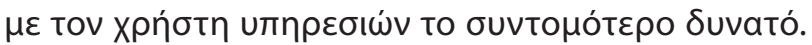

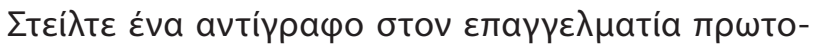

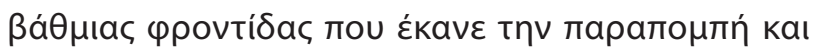

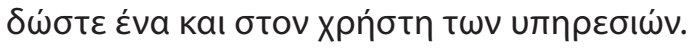

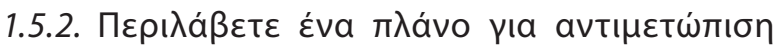

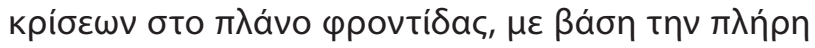

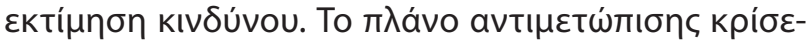

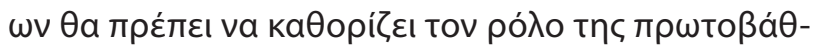

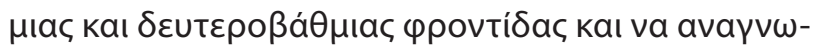

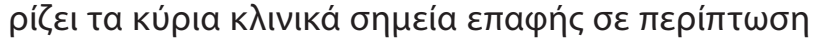

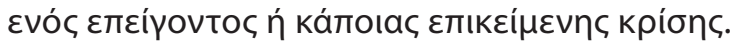

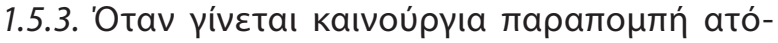

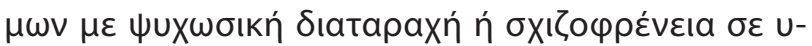

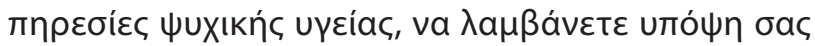

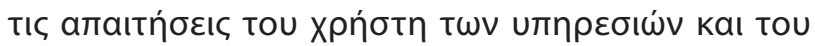

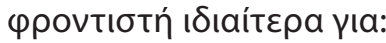

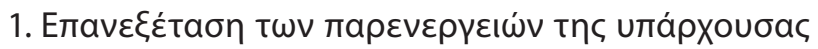
Өвратві́ac.

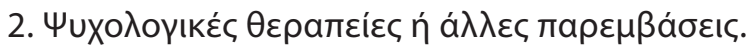

1.5.4. 'Otav үívetaı кámoı тактІкń паратонти́ a-

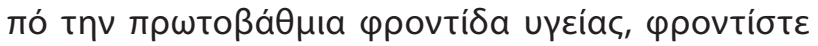

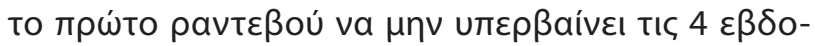
$\mu a ́ \delta \varepsilon \varsigma$.

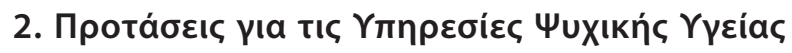

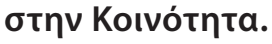

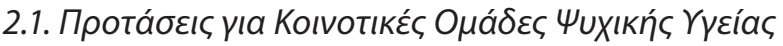

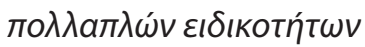

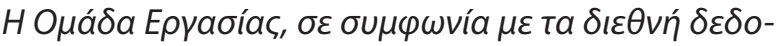

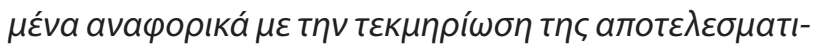

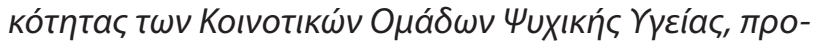

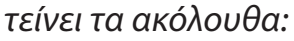

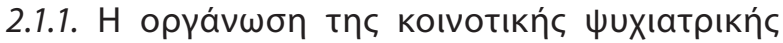

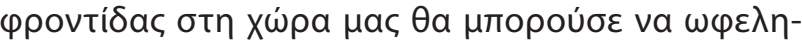

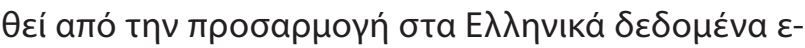

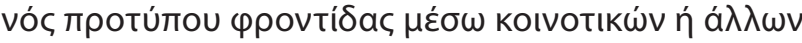

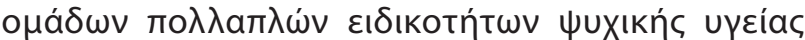

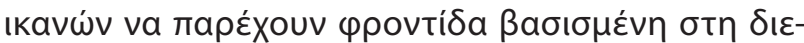
$\theta v \eta \dot{~ « к а \lambda ท ́ ~ п р а к т і к n ́ » . ~}$

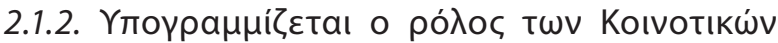

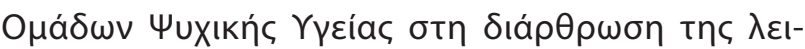

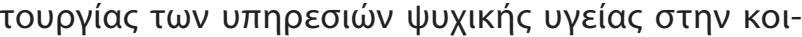

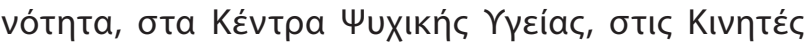

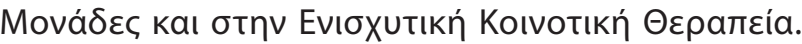

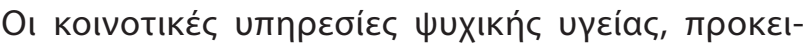

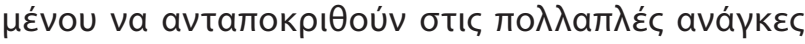

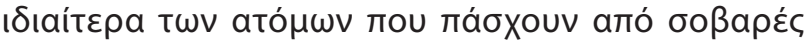

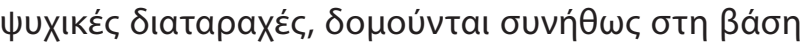

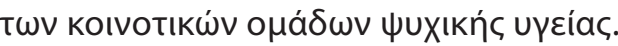

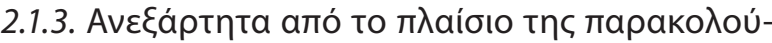

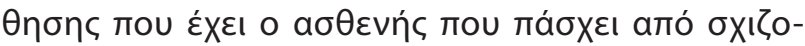

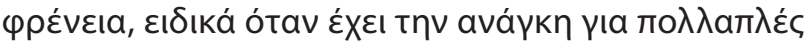

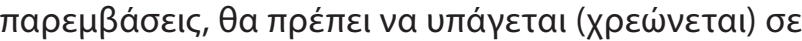

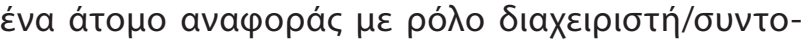

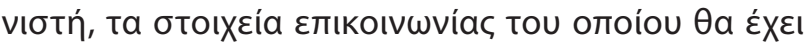

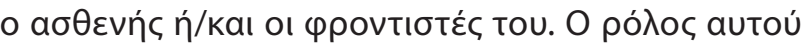

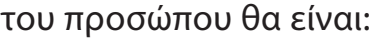

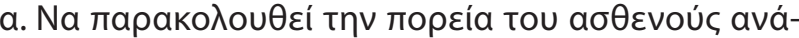

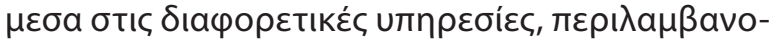

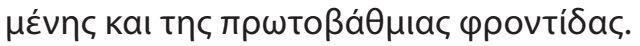

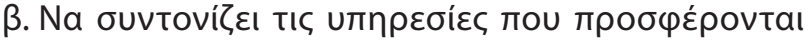

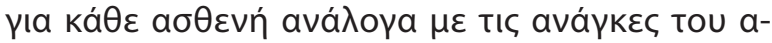

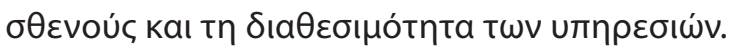

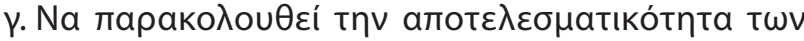

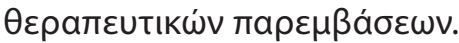

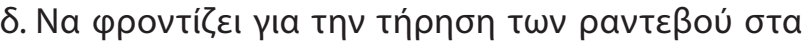

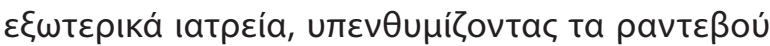

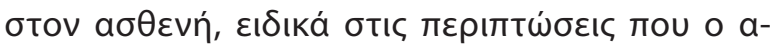

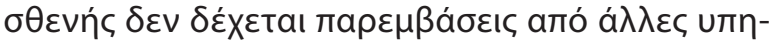

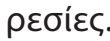

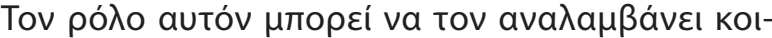

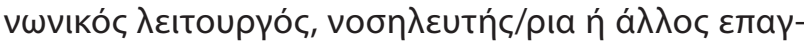

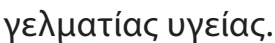

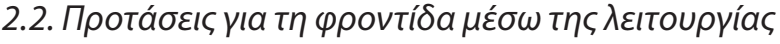

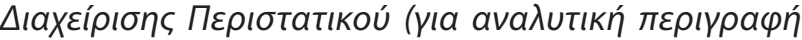

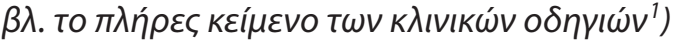




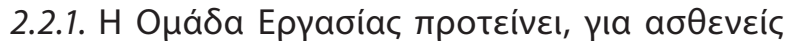

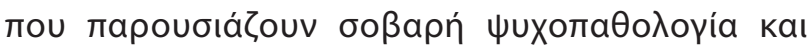

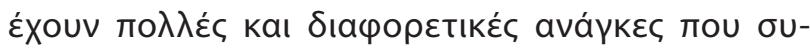

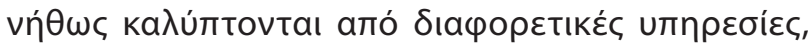

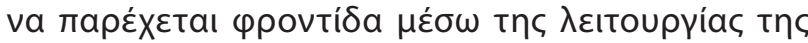

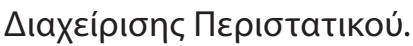

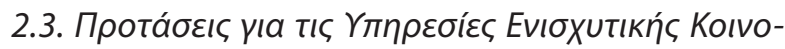

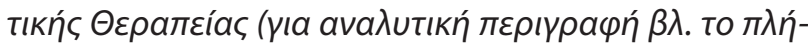

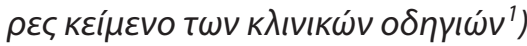

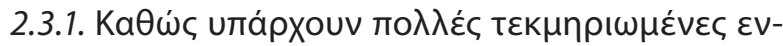

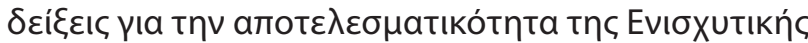

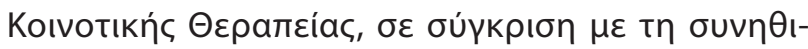

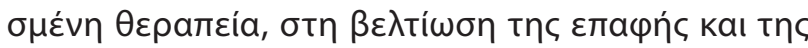

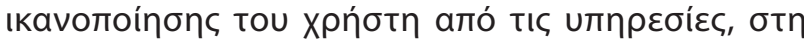

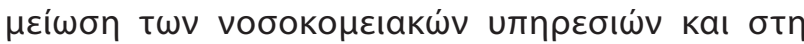

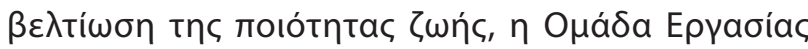

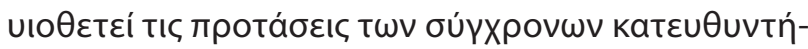

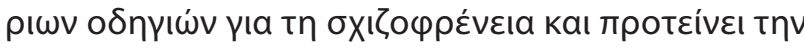

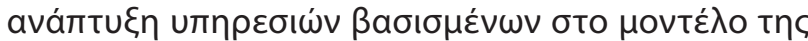

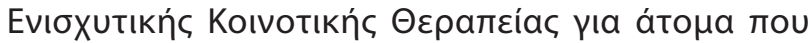

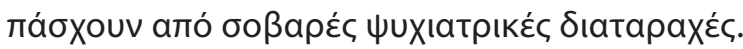

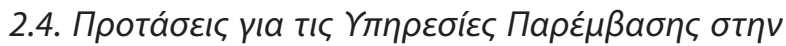

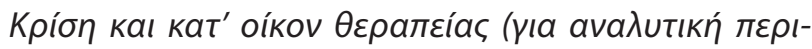

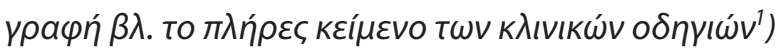

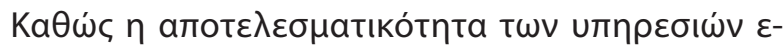

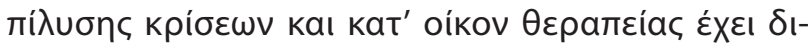

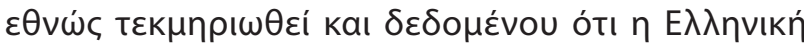

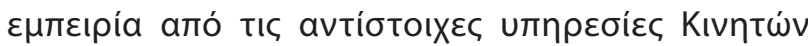

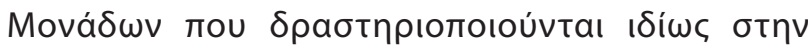

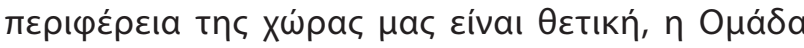

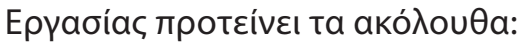

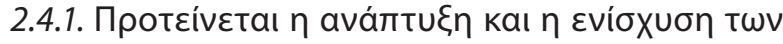

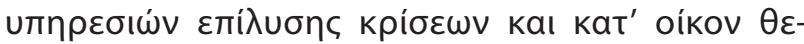

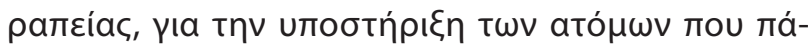

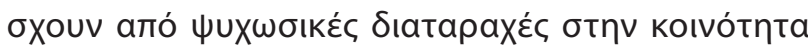

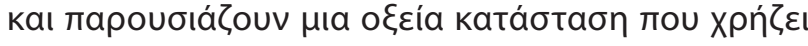

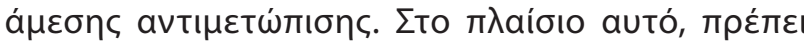

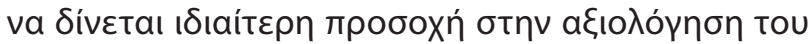

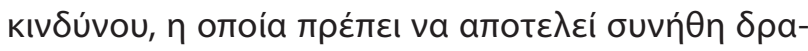

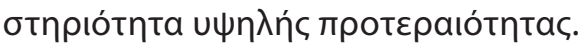

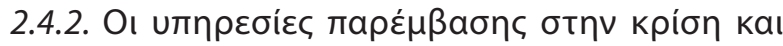

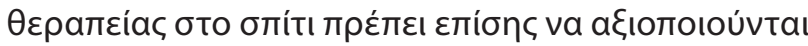

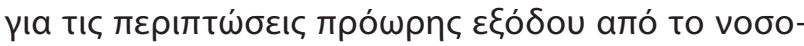
концío.

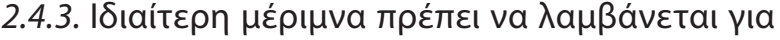

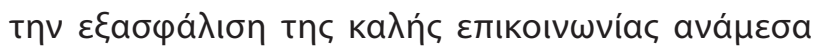

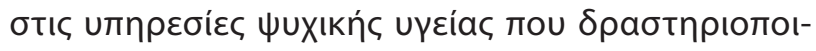

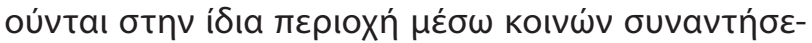

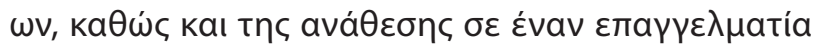

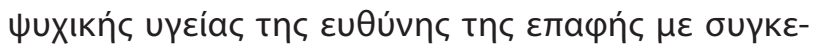

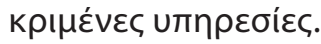

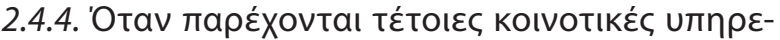

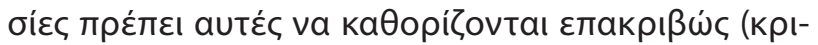

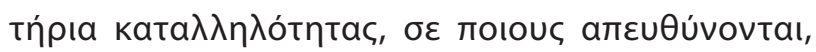

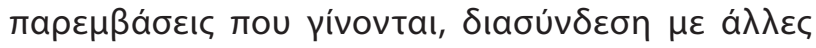

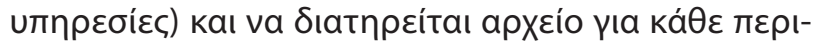

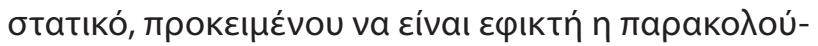

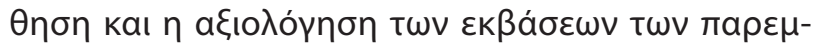

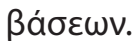

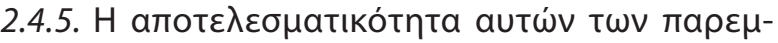

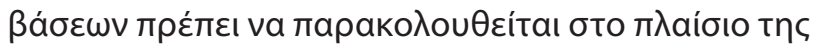

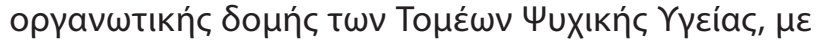

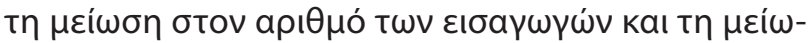

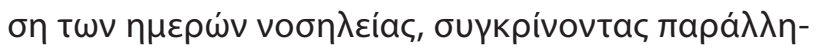

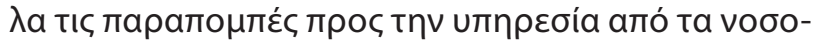

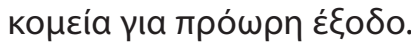

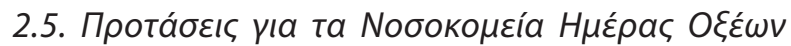
Пврібтатіки́v

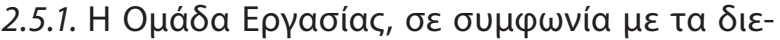

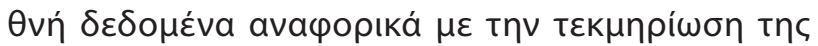

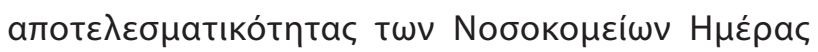

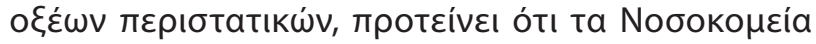

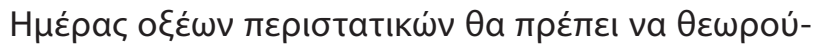

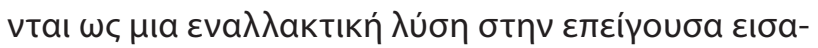

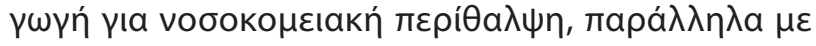

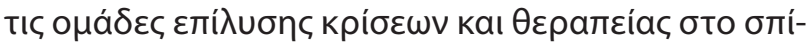

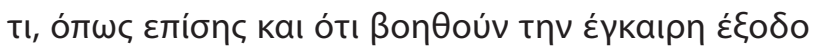

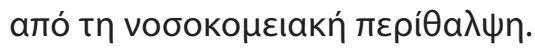

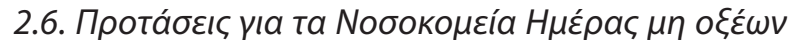
пврıбтатıки́v

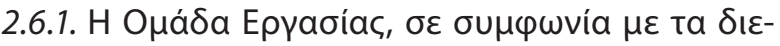

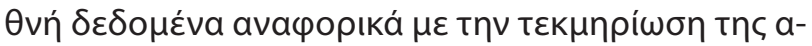

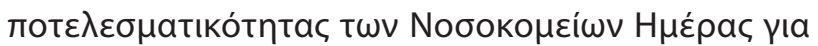

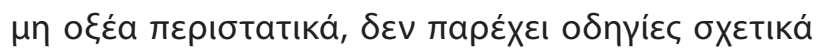

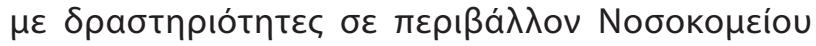

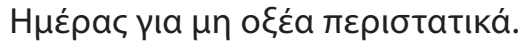




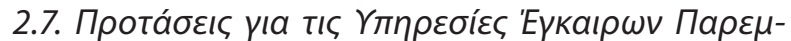

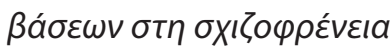

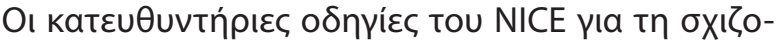

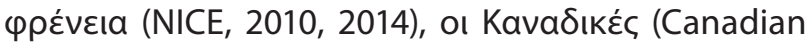

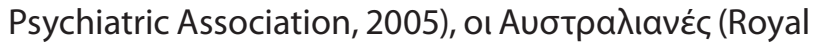
Australian and New Zealand College of Psychiatrists,

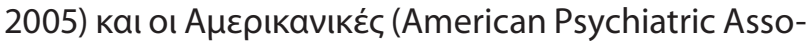

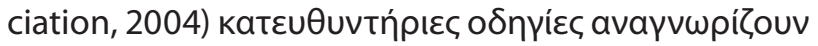

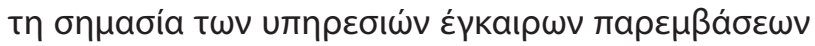

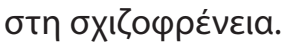

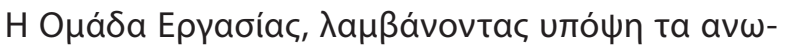

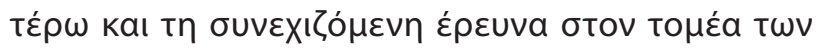

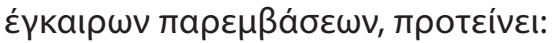

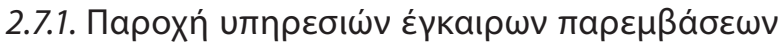

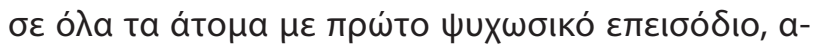

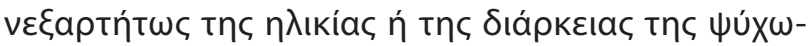

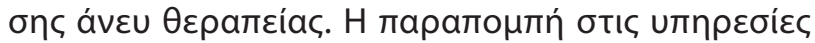

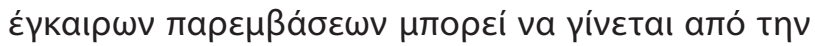

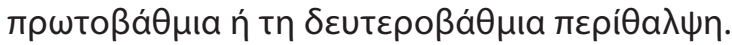

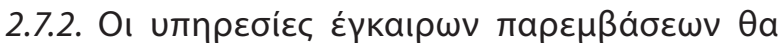

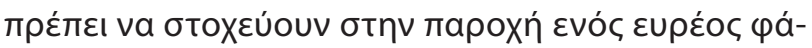

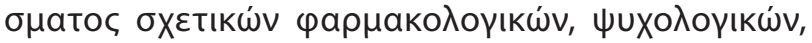

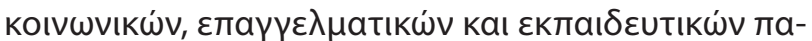

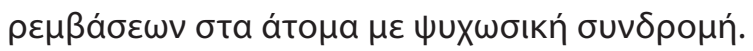

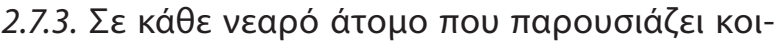

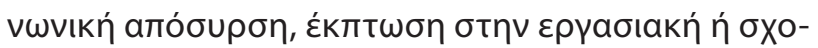

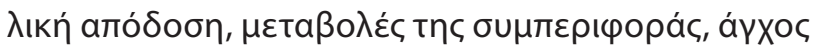

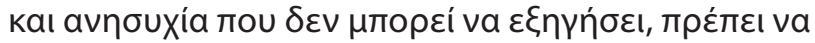

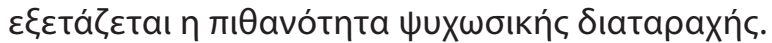

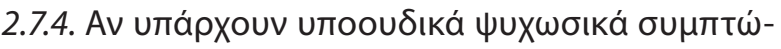

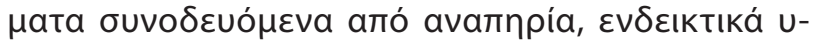

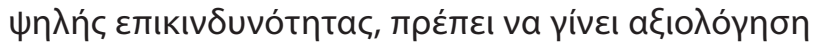

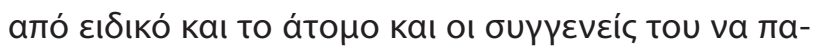

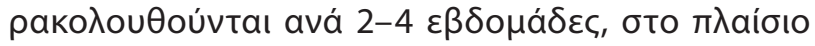

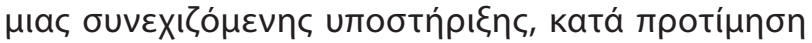

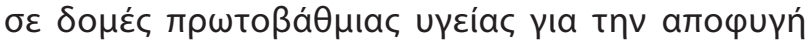

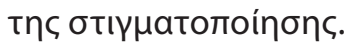

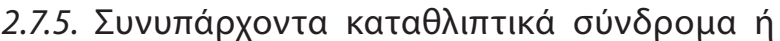

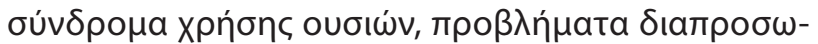

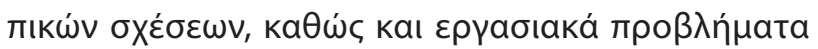

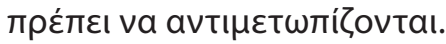

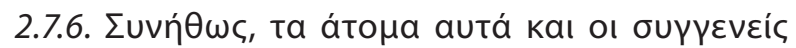

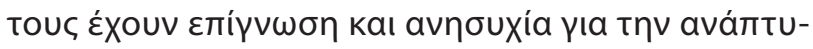

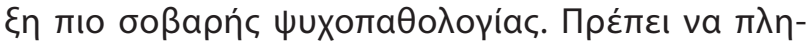

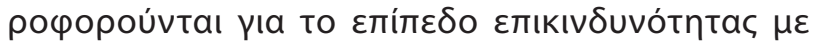

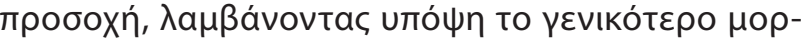

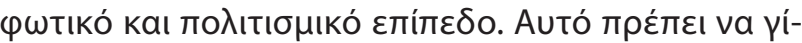

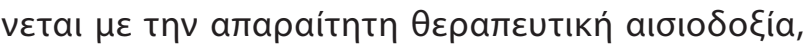

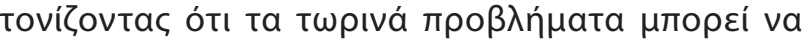

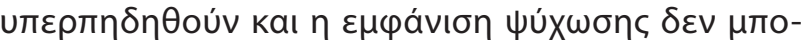

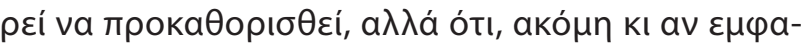

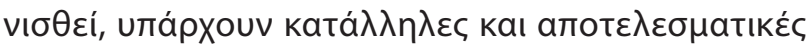
Ө

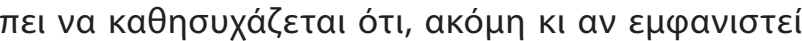

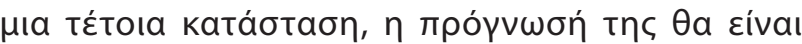

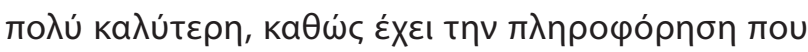

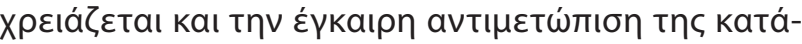
otaons.

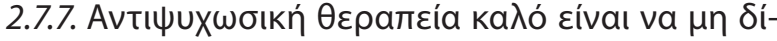

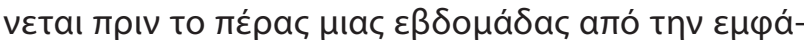

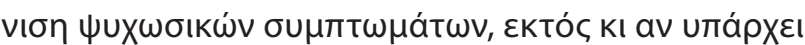

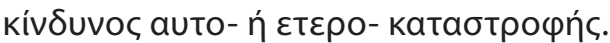

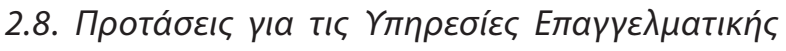
Атоката́бтабпৎ

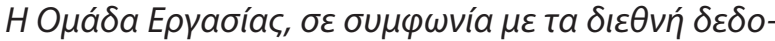

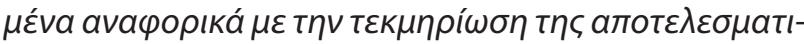

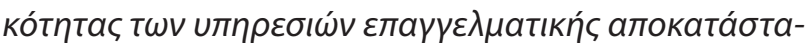

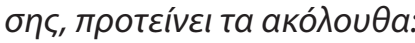

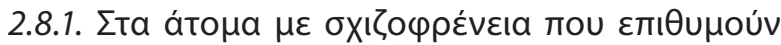

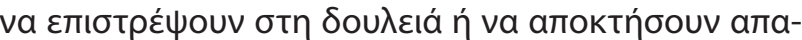

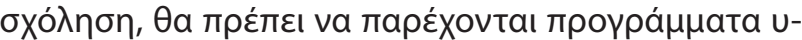

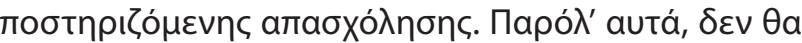

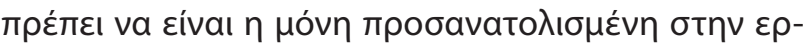

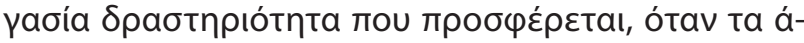

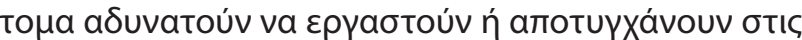

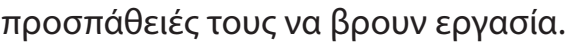

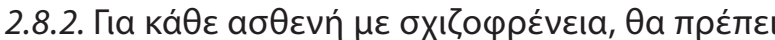

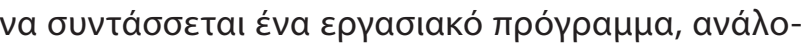

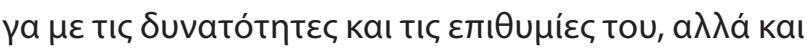

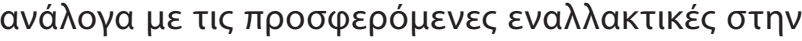

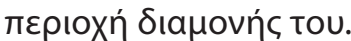

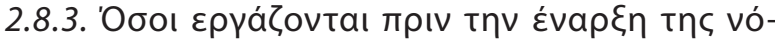

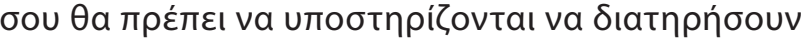

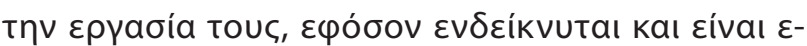
$\varphi$ IKTó.

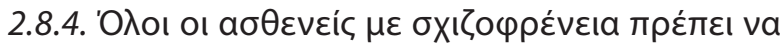

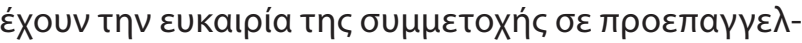

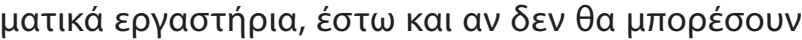

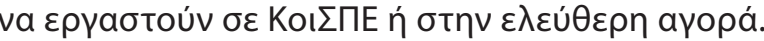




\title{
Clinical guidelines for the management of schizophrenia (II): Community service-level interventions and the role of Primary Care
}

\author{
M. Margariti, ${ }^{1}$ M. Hadjulis, ${ }^{2}$ M. Lazaridou, ${ }^{3}$ G.F. Angelidis, ${ }^{4}$ \\ V. Fotopoulos, ${ }^{5}$ L. Markaki, ${ }^{6}$ F. Koulouri ${ }^{7}$ \\ ${ }^{1} 1$ st Department of Psychiatry, Medical School, National and Kapodistrian University of Athens, \\ ${ }^{2}$ Department of Psychiatry, "Agioi Anargyroi" Hospital, Faculty of Nursing, National \& Kapodistrian University of Athens, \\ ${ }^{3}$ Society of Social Psychiatry and Mental Health, Athens, \\ ${ }^{4}$ Department of Psychiatry, General Hospital of Katerini, Katerini \\ ${ }^{5}$ Dromokaiteio Mental Hospital, Athens, ${ }^{6}$ Executive Council, Hellenic Psychiatric Association, Athens, \\ ${ }^{7}$ Directorate of Mental Health, Ministry of Health, Athens, Greece
}

Psychiatriki 2018, 29:118-129

Schizophrenia is one of the most disabling disorders globally, with a significant impact on the professional, social and personal functioning of those affected. Mortality rates are estimated to be 2-2.5 times greater than in the general population attributable to not only suicide but also physical illnesses, such as cardiovascular, metabolic and infectious diseases. Patients with schizophrenia have increased needs for health services which vary according to the stage of the illness and the way the disorder affects the patient. A significant part of the costs of treating patients with schizophrenia is attributed to the health service costs, both to address the disorder itself and its wider effects. In 2015 the National Clinical Guidelines for the management of schizophrenia were formulated by a Working Group that was set up by the Greek Ministry of Health. In this article, a summary of the recommendations (as included in the National Clinical Guidelines) is presented, describing the role of primary care and community mental health services in the management of schizophrenia. The NICE Guideline (National Institute of Clinical Excellence, 2010, 2014) for the management of Psychosis \& Schizophrenia was utilized as the main guide to develop the Greek National Guidelines . In addition the American Psychiatric Association (APA) Practice Guideline for the Treatment of Patients with Schizophrenia (APA, 2004), the Royal Australian and New Zealand College of Psychiatrists clinical practice guidelines for the treatment of schizophrenia and related disorders (Royal Australian and New Zealand College of Psychiatrists, 2005), as well as other relevant sources were also used. The Working Group also took into consideration the available Greek bibliography as well as the external evaluations of the Greek psychiatric reform programs. Special effort was made to adapt the international experience to the current Greek landscape with its constraints resulting from the uneven dispersion of mental health services, the lack of coordination between services, the incomplete sectorization of mental health services provided as part of the National Health System, the still underdeveloped Primary Care Health Service, and last but not least the difficult economic situation in Greece. The proposals aim to contribute to the efficient implementation of the provision of community mental health services for patients suffering from psychotic disorders, focusing in particular on: (a) the role of primary health care services and the role of family physician in the treatment of schizophrenia; (b) the cooperation and coordination between mental health services and primary care services in order to ensure continuity of care (c) designing services that deliver evidence-based 
interventions,thus ensuring that a larger proportion of the population receives interventions with documented effectiveness in the treatment of schizophrenia, in the least restrictive environment. A particular emphasis is placed on services that are expected to reduce the need for hospitalization, such as crisis intervention services, community interventions aimed at maintaining and continuing the treatment of difficult patients who tend to be lost to follow up from services and early intervention services in psychosis.

Key words: Clinical guidelines, treatment guidelines, schizophrenia, primary health care, community psychiatry, mental health services.

\section{Bıßลtoypacpía}

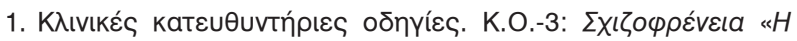

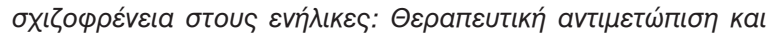

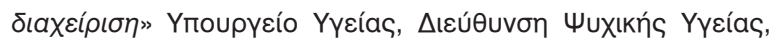
AӨńva, 2015

2. Margariti M, Hadjulis M, Lazaridou M, Angelidis GF, Fotopoulos $\mathrm{V}$, Markaki L, Koulouri F. Clinical guidelines for the management of schizophrenia: Aims and limitations (I), Psychiatriki 2017, 28:301-305

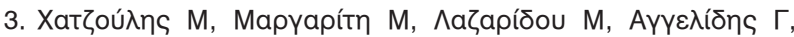

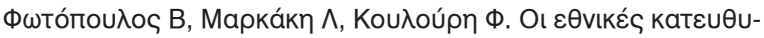

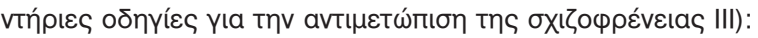

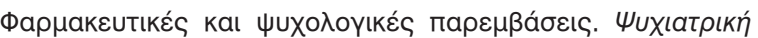

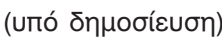

4. Jablensky A, Sartorius N, Ernberg G et al. Schizophrenia: manifestations, incidence and course in different cultures. A World Health Organization ten country study. Psychol Med Monogr Supp/ 1992, 20:1-97, PMID:1565705

5. Jobe TH, Harrow M. Long-term outcome of patients with schizophrenia: a review. Can J Psychiatry 2005, 50:892-900, doi: 10.1177/070674370505001403

6. Thornicroft G, Tansella M. What are the Arguments for CommunityBased Mental Health Care? Copenhagen: WHO Regional Office for Europe Health Evidence Network report, 2003, http://www. euro.who.int/document/E82976.pdf (accessed 6 June 2011).

7. Hoult J. Developing the South Islington Crisis Resolution Team. Ment Health Rev J 1999, 4:14-16, doi: 10.1108/136193 22199900030

8. McGlynn P, Flowers M. Working through a crisis: the process of crisis resolution. In: Glynn P (ed) Crisis Resolution and Home Treatment: A Practical Guide. London, Sainsbury Centre for Mental Health, 2006:65-76

9. Marshall M, Lockwood A. Assertive community treatment for people with severe mental disorders. (Cochrane Review). The Cochrane Library, Issue 3. Oxford, Update Software, 2002

10. McGrew JH, Bond GR. Critical ingredients of assertive community treatment: judgments of the experts. $J$ Ment Health Adm 1995, 22:113-125, PMID:10142125

11. Linszen D, Dingemans $P$, Lenoir $M$. Early intervention and a five-year follow-up in young adults with a short duration of untreated psychosis: ethical implications. Schizophr Res 2001, 51:55-61, PMID:11479066

12. Marshall M, Crowther R, Almaraz-Serrano A, Creed F, Sledge $\mathrm{W}$, Kluiter $\mathrm{H}$ et al. Systematic reviews of the effectiveness of day care for people with severe mental disorders: (1) acute day hospital versus admission; (2) vocational rehabilitation; (3) day hospital versus outpatient care. Health Technol Assess 2001, 2:1-75, PMID: 11532238

13. Christodoulou G. Psychiatric reform revisited. World Psychiatry 2009, 8:121-122, PMID: 19516938

14. Christodoulou G, Ploumpidis D, Christodoulou N, Anagnostopoulos D. The State of Psychiatry in Greece. Int Rev Psychiatry 2012, 24:301-306, doi: 10.3109/09540261.2012. 691874

15. Douzenis A, Michopoulos I, Economou M, Rizos E, Christodoulou C, Lykouras L. Involuntary admission in Greece: a prospective national study of police involvement and client characteristics affecting emergency assessment. Int J Soc Psychiatry 2010, 58:172-177, doi:10.1177/0020764010387477

18. Economou, M, Richardson C, Gramandani C et al. Knowledge About Schizophrenia and Attitudes Towards People with Schizophrenia in Greece. Int J Soc Psychiatry 2009, 55:361-371, doi : 10.1177/0020764008093957

17. Loukidou E, Bouras N. Greek mental health reforms: views and perceptions of professionals and service users. Psychiatriki 2013, 24:39-46, PMID: 23603267

18. Loukidou E \& Thornicroft G. Evaluation of Greek Psychiatric Reforms: Methodological Issues. Intern J Ment Health System 2013, 7:1-8, doi: 10.1186/1752-4458-7-11

19. American Psychiatric Association. Practice Guideline for the Treatment of Patients with Schizophrenia. Washington, DC: American Psychiatric Association 2004 http://psychiatryonline. org/pb/assets/raw/sitewide/practice_guidelines/guidelines/ schizophrenia.pdf (accessed 6 June 2014)

20. Canadian Psychiatric Association. Clinical Practice Guidelines treatment of schizophrenia. Can J Psych 2005, 50:(Suppl) 1, PMID: 16529334

21. NICE. The NICE Guideline on Core Interventions in the Treatment and Management of Schizophrenia in Adults in Primary and Secondary Care. NICE Clinical Guideline no. 82. 
London, National Institute of Health and Clinical Excellence, 2009

22. NICE. Psychosis and schizophrenia in adults: treatment and management. NICE Clinical Guideline no. 178. London: National Institute of Health and Clinical Excellence, 2014

23. Royal Australian and New Zealand College of Psychiatrists Clinical Practice Guidelines Team for the Treatment of Schizophrenia and Related Disorders. Royal Australian and New Zealand College of Psychiatrists clinical practice guidelines for the treatment of schizophrenia and related disorders. Aust $N$ Z J Psychiatry 2005, 39:1-30, doi: 10.1177/0004867416641195

24. DeHert M, Vancampfort D, Correll C, Mercken V, Peuskens J, Sweers $\mathrm{K}$ et al. Guidelines for screening and monitoring of cardiometabolic risk in schizophrenia: systematic evaluation. $\mathrm{Br} \mathrm{J}$ Psychiatry 2011, 199:99-105, doi: 10.1192/bjp.bp.110.084665

25. Drake ER, Goldman HH, Leff HS, Dixon L, Mueser KT, Torrey WC. Implementing Evidence-Based practices in Routine mental Health Service Settings. Psychiatr Serv 2001, 52:179-182, doi: 10.1176/appi.ps.52.2.179

26. Lehman AF, Steinwachs DM \& the co-investigators of the PORT Project. Translating research into practice: the Schizophrenia Patient Outcomes Research Team (PORT) treatment recommendations. Schizophr Bull 1998a, 24:1-10, PMID: 9502542
27. Thornicroft G, Alem A, Dos Santos R, Barley A et al. WPA guidance on steps, obstacles and mistakes to avoid in the implementation of community mental health care. World Psychiatry 2010, 9:67-77, PMCID: PMC2911080

28. Lehman AF, Steinwachs DM \& PORT Co-investigators. Patterns of usual care for schizophrenia: initial survey results from the Schizophrenia Patient Outcomes Research Team (PORT) survey. Schizophr Bull 1998b, 24:11-20, PMID: 9502543

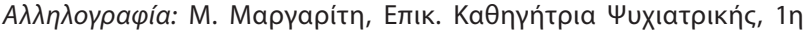

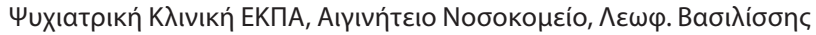

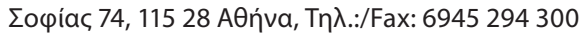

e-mail: marimargariti@gmail.com, mmarg@med.uoa.gr 


\title{
Eıठııó á $\rho \theta \rho o$ Special article
}

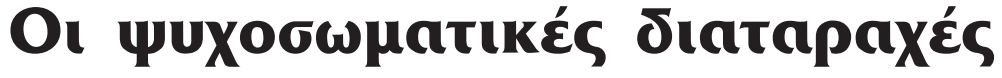

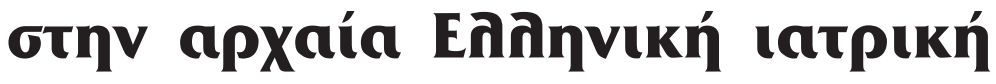

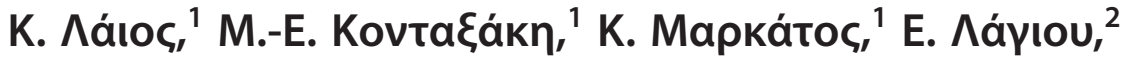

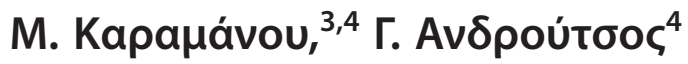

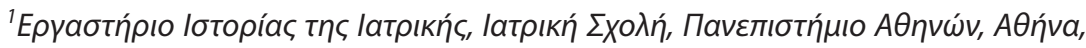

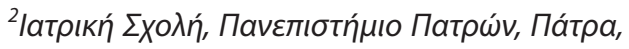

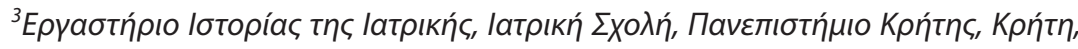

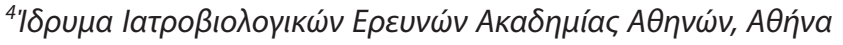

Uuxıatpıkń 2018, 29:130-136

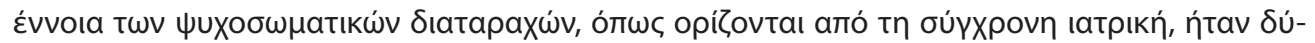

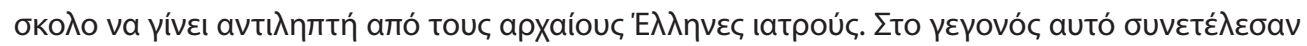

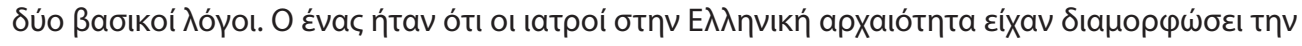

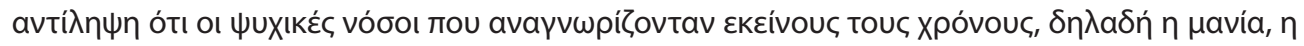

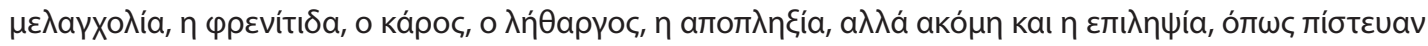

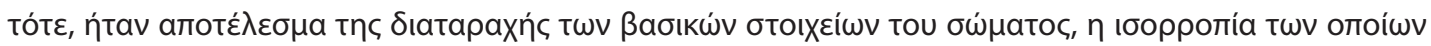

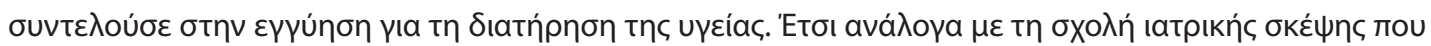

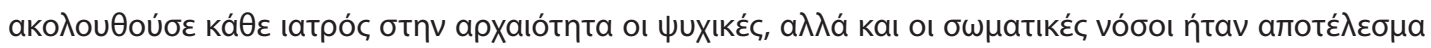

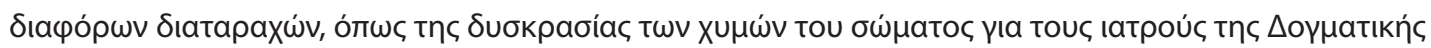

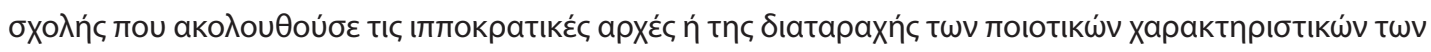

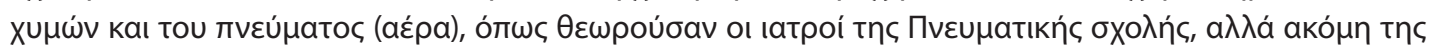

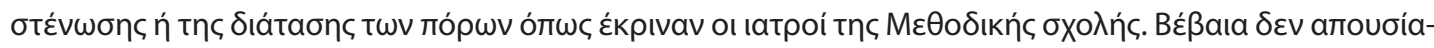

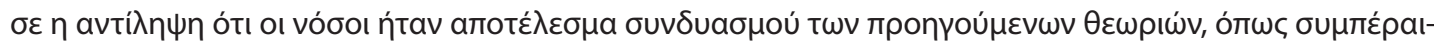

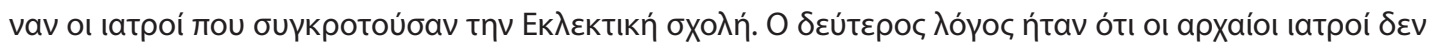

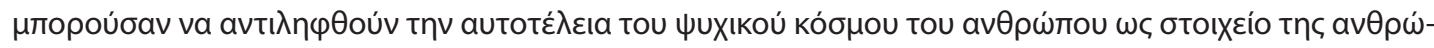

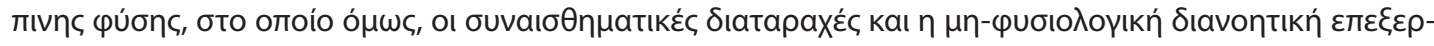

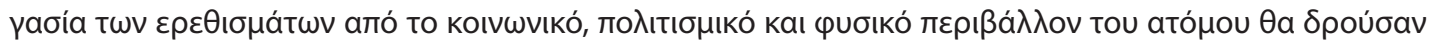

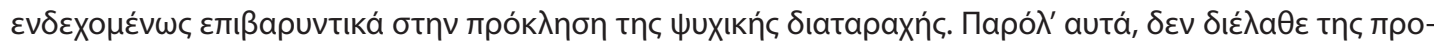

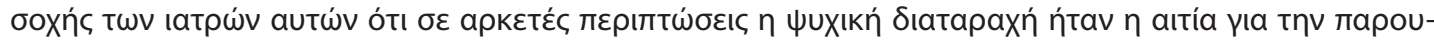

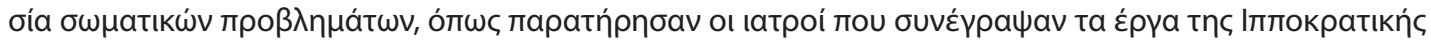

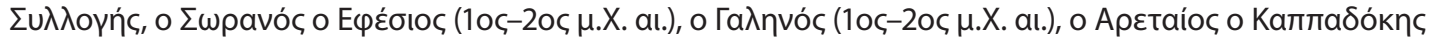




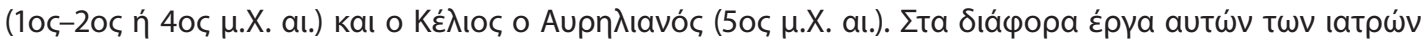

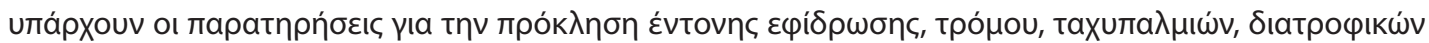

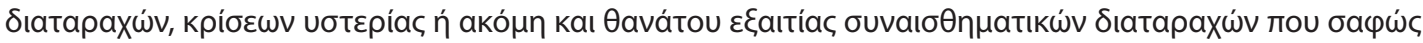

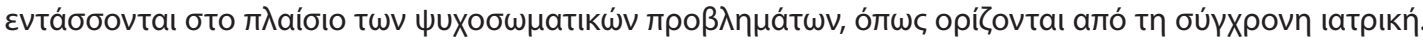

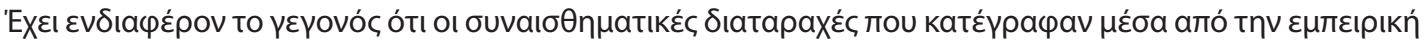

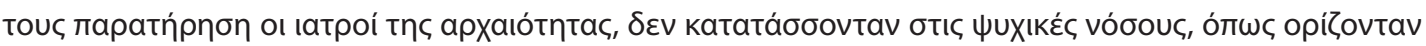

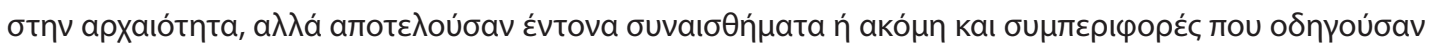

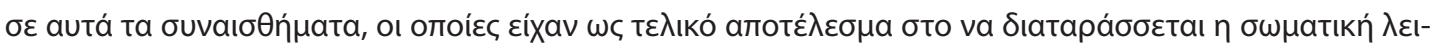

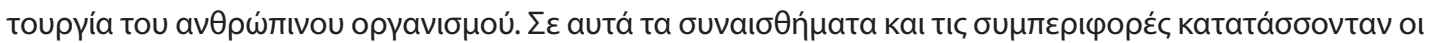

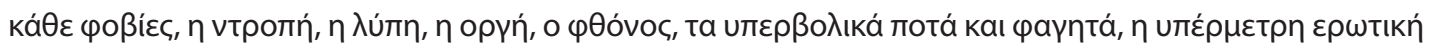

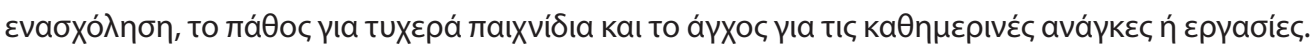

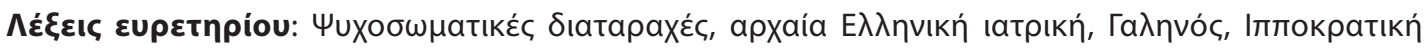
$\Sigma \cup \lambda \lambda o \gamma \eta \dot{. ~}$

\section{Eıaywyń}

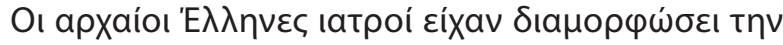

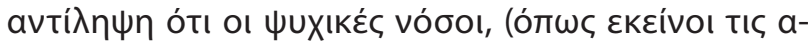

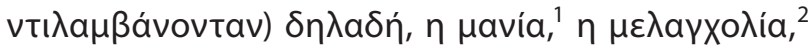

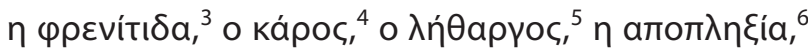

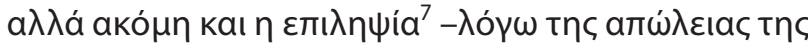

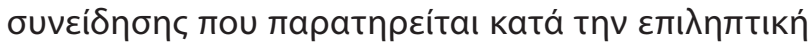

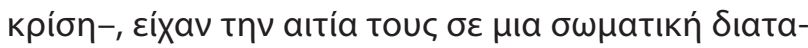

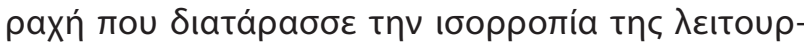

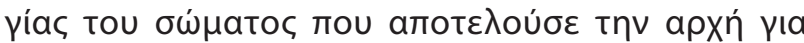

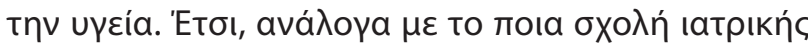

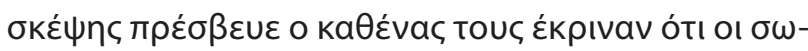

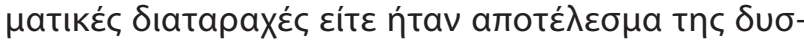

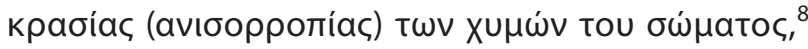

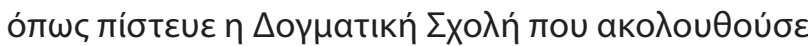

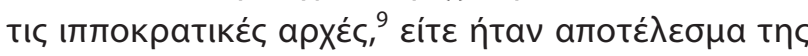

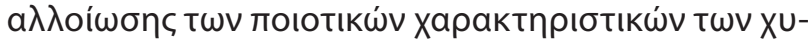

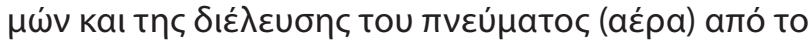

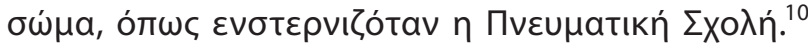

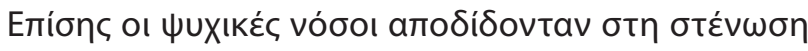

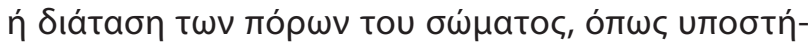

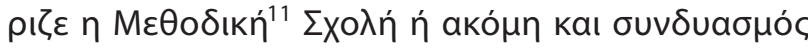

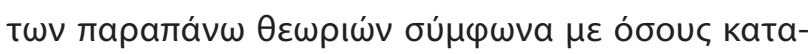

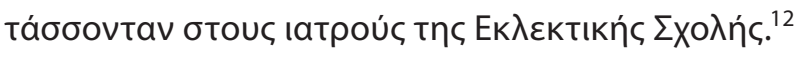

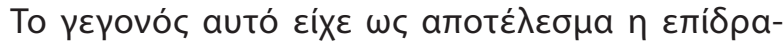

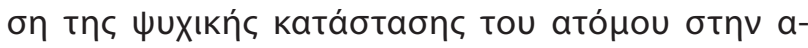

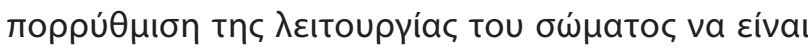

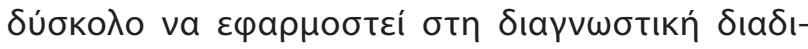

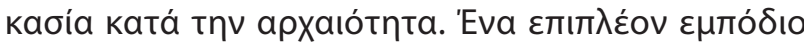

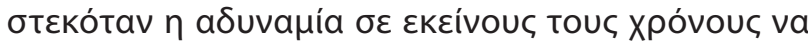

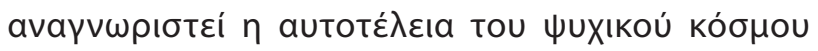

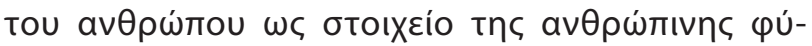

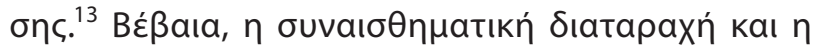

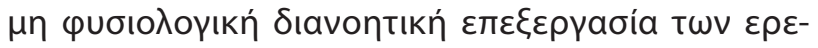

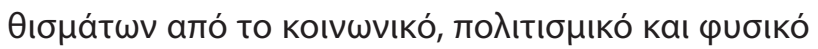

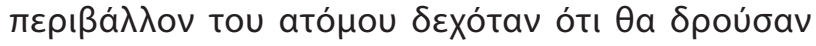

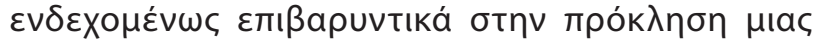

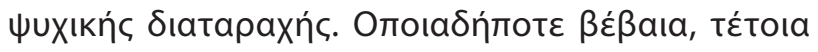

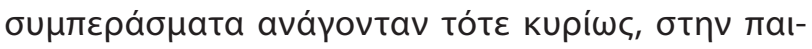

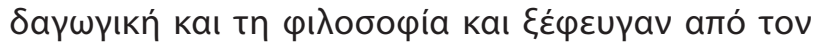

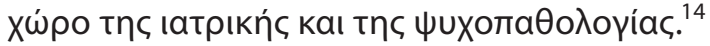

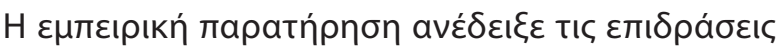

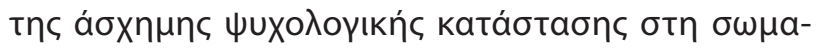

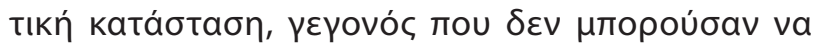

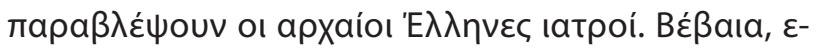

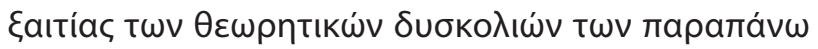

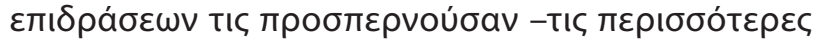

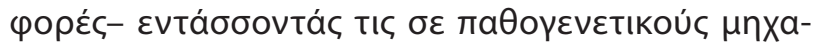

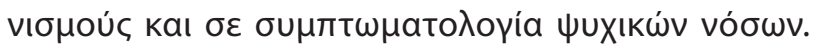

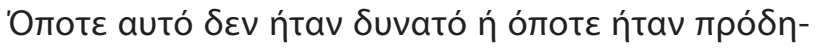

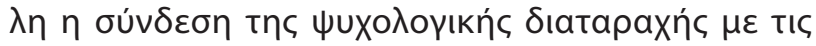

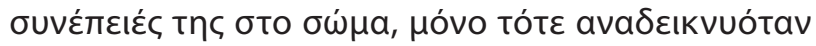

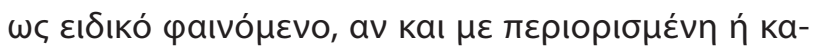

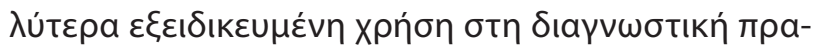

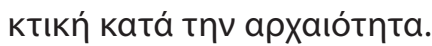

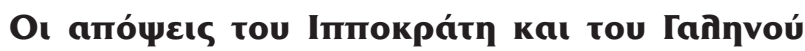

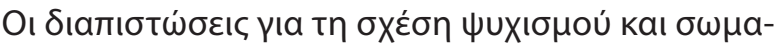

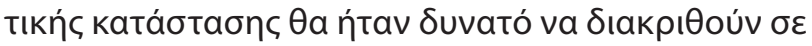

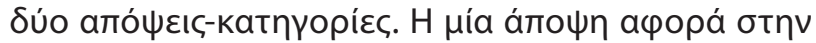




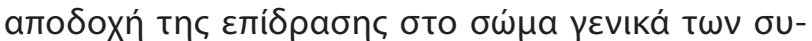

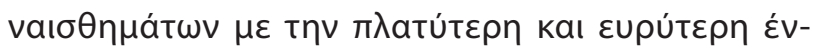

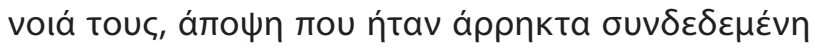

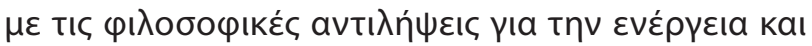

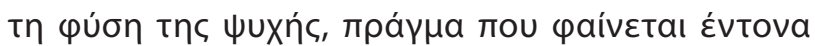

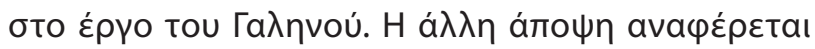

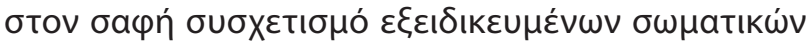

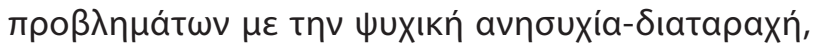

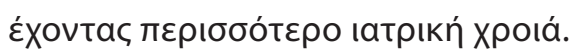

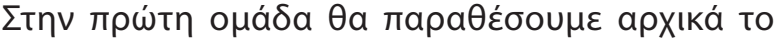

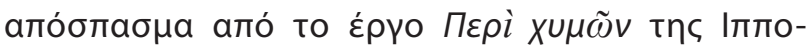

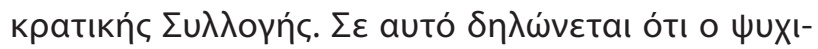

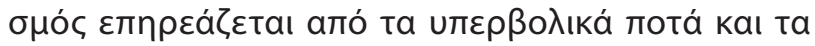

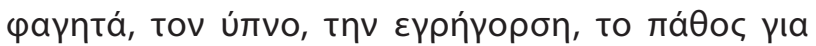

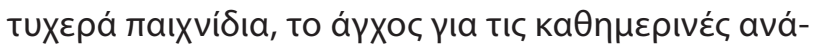

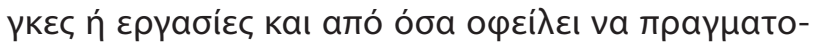

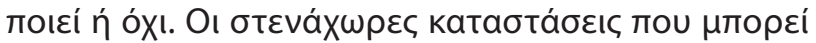

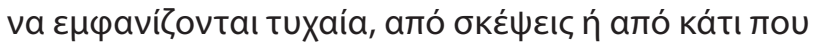

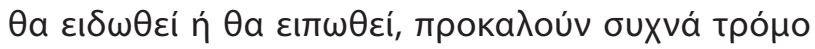

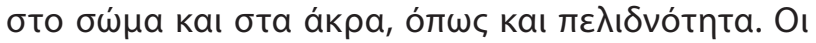

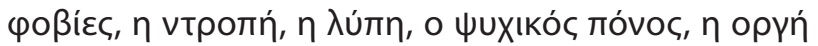

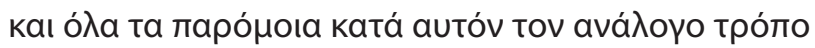

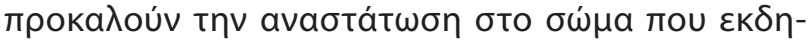

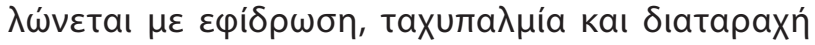

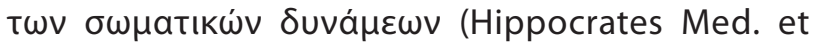
Corp. De humoribus 9.1-13). ${ }^{15}$

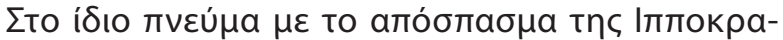

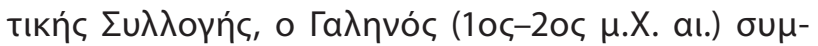

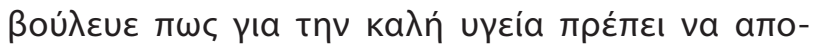

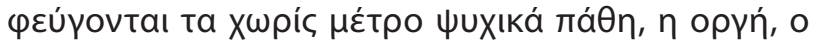

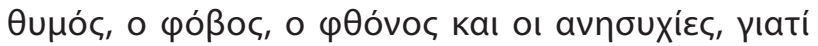

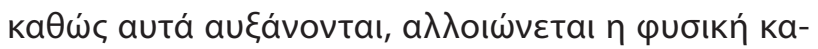

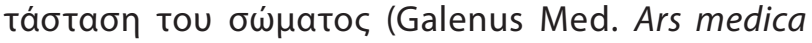
1.371.10-14). ${ }^{16}$

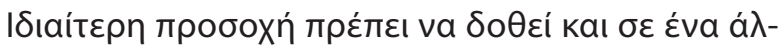

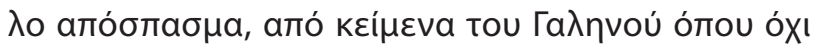

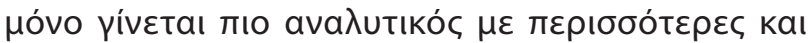

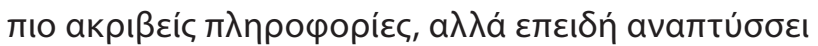

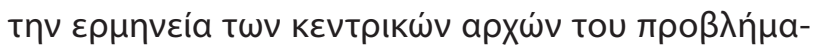

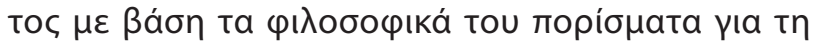

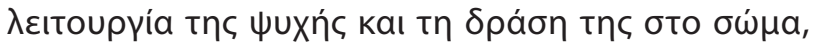

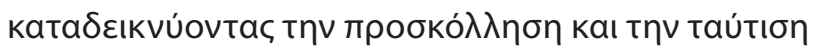

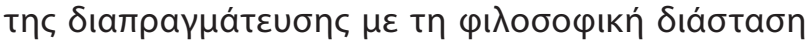

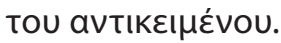

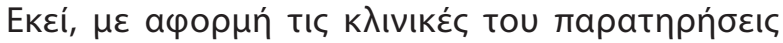

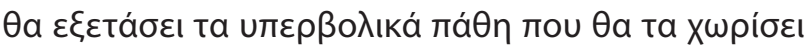

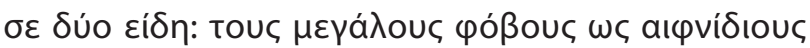

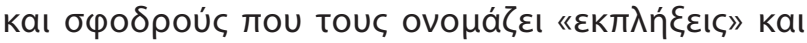

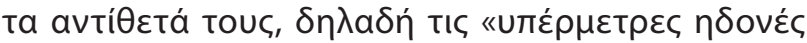

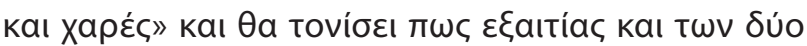

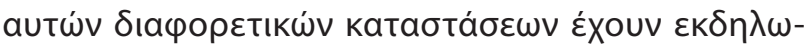

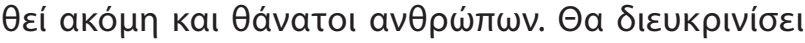

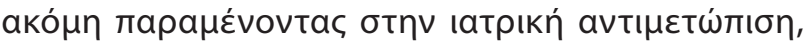

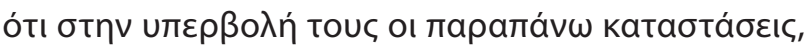

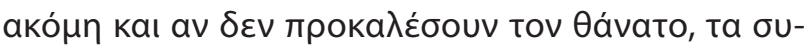

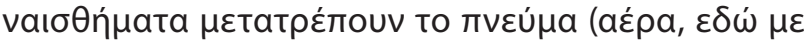

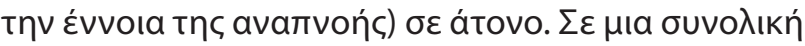
атоті́

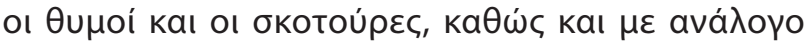

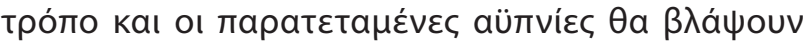

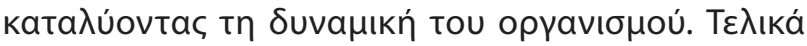

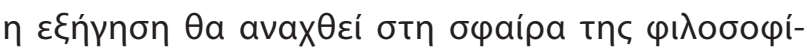
ac, a

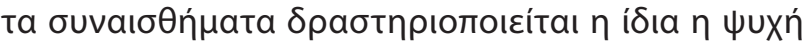

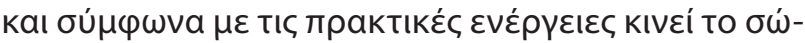
$\mu a$,

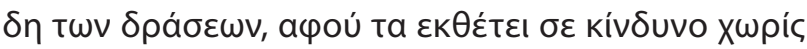
$\mu \varepsilon ́ t \rho o$ (Galenus Med. De methodo medendi libri xiv 10.841.8-10.842.2). ${ }^{17}$

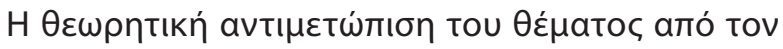

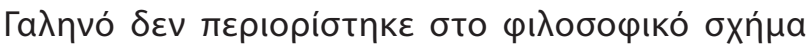

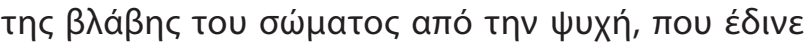

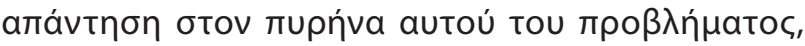

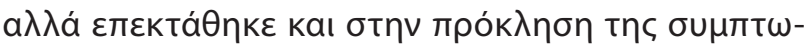

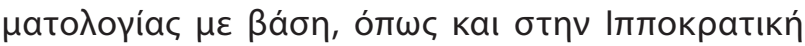

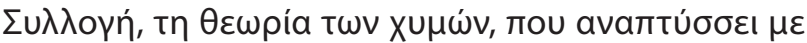

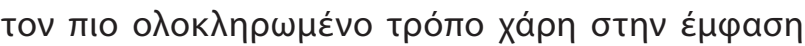

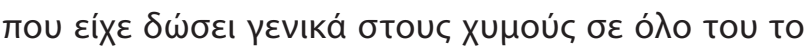
غ́рүo.

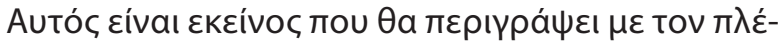

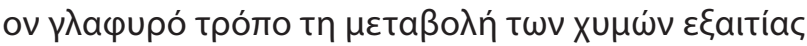

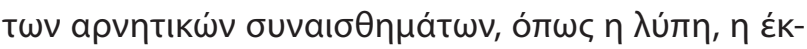

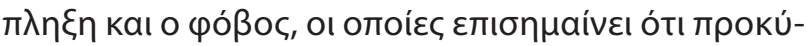

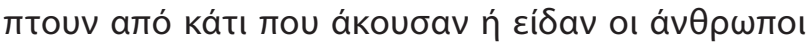
(Galenus Med. De sanitate tuenda libri vi 6.277.146.278.12, Galenus Med. De symptomatum causis libri iii 7.191.4-7.193.18). ${ }^{18,19}$

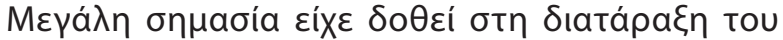

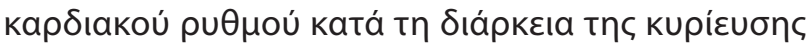




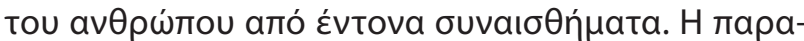

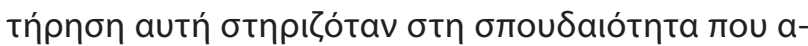

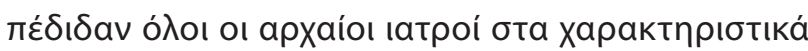

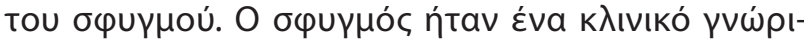

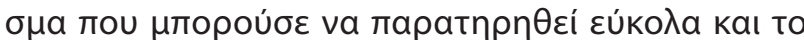

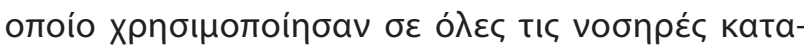

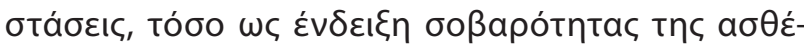

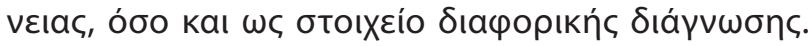

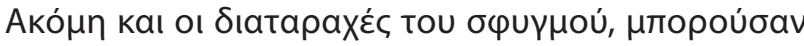

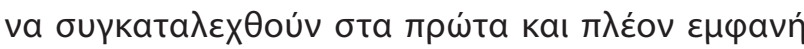

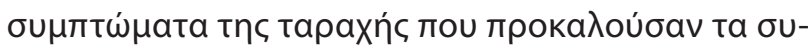

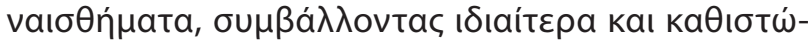

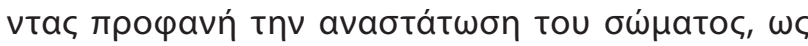

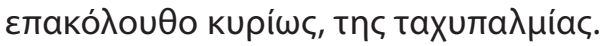

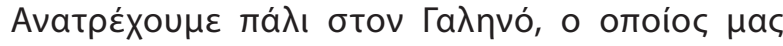

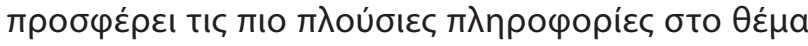

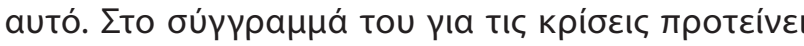

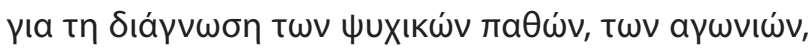

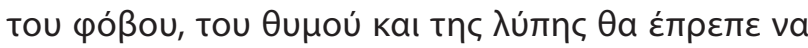

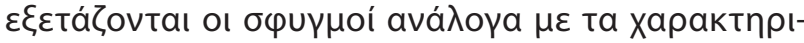

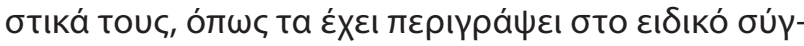
үранนа үı' autoúc (Galenus Med. De crisibus libri iii 9.696.16-9.697.5). ${ }^{20}$

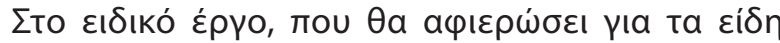

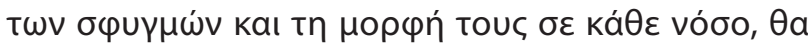

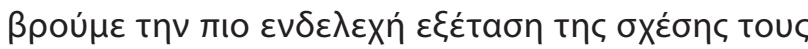

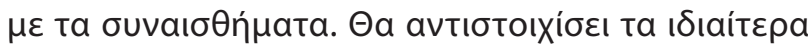

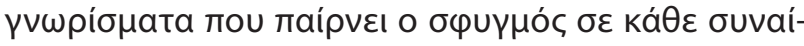

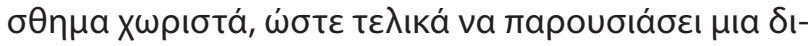

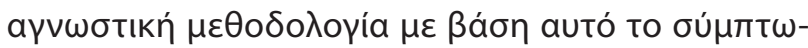

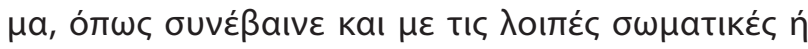

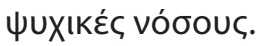

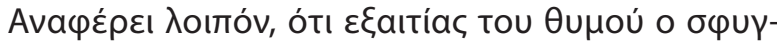

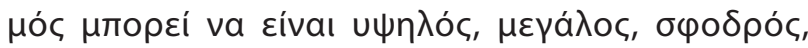

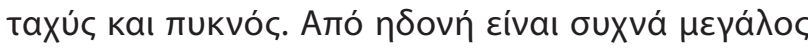

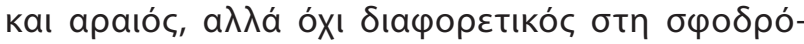

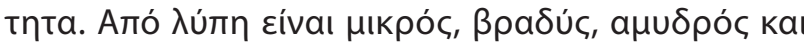

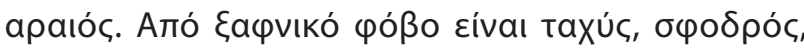

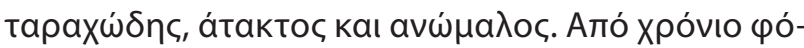

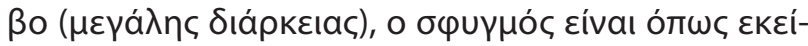

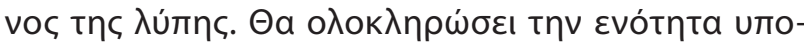
$\delta \varepsilon$ ккvи́ovtac tov тро́то $\mu \varepsilon$ tov отоі́ катаßá $\lambda \lambda \varepsilon т a ı$

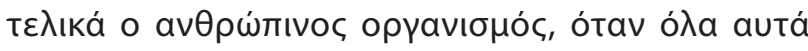

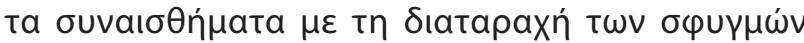

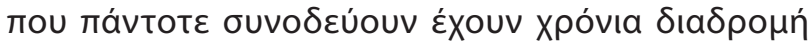

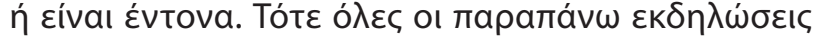

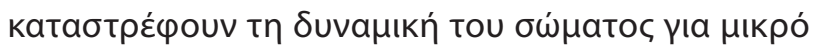

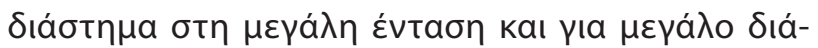

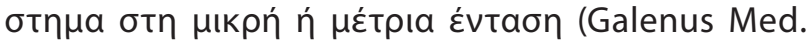
De pulsibus libellus ad tirones 8.473.13-8.474.4). To

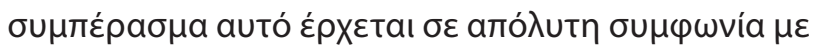

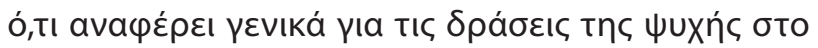
$\sigma \omega ́ \mu a$ (Galenus Med. De methodo medendi libri xiv 10.841.8-10.842.2). ${ }^{21}$

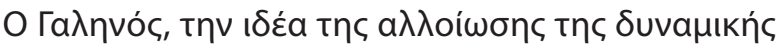

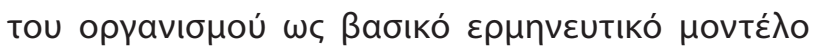

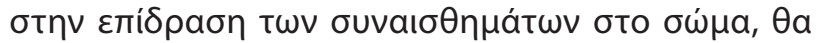

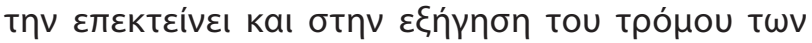

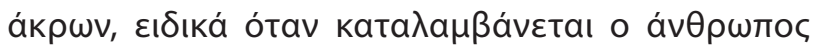

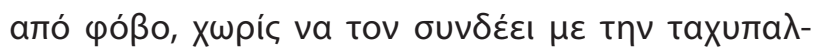

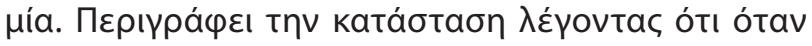

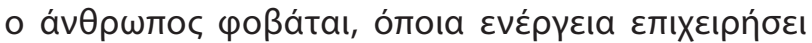

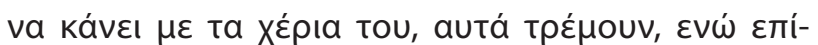

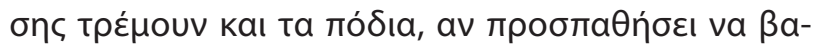

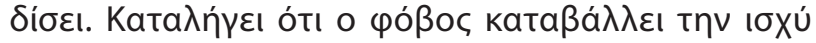

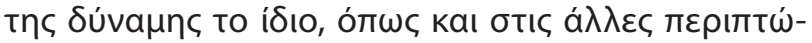

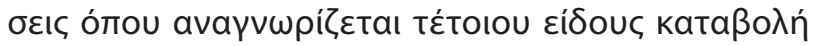
(Galenus Med. De tremore, palpitatione, convulsione et rigoreliber 7.587.6-10). ${ }^{22}$

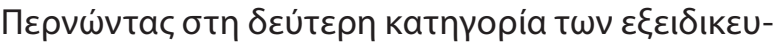

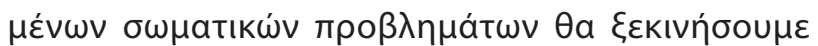

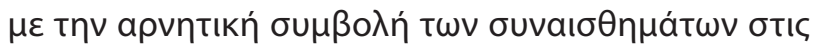

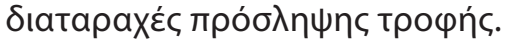

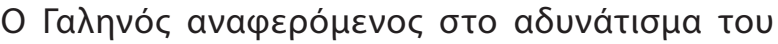

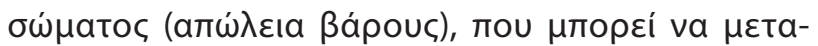

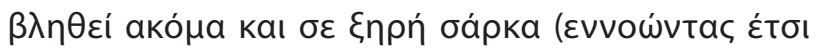

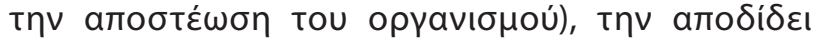

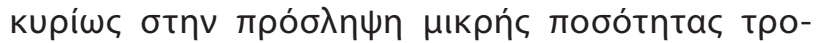

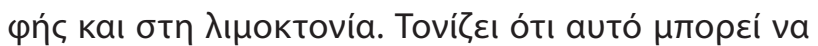

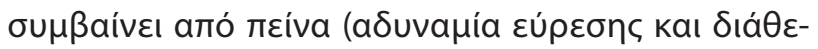

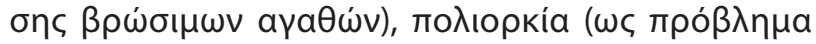

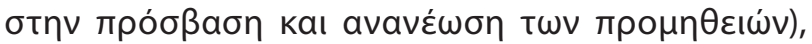

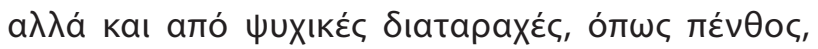

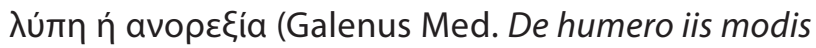
prolapso quos Hippocrates non vidit 18a.362.12-15). ${ }^{23}$

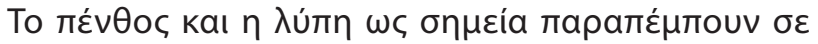

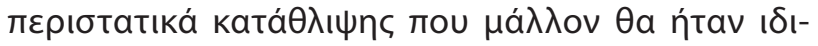

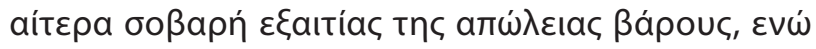

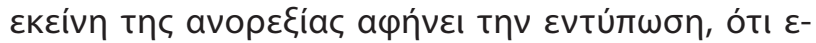

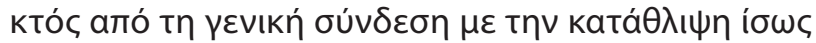


a

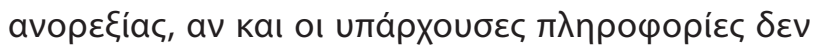

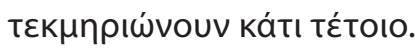

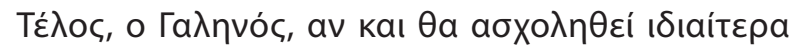

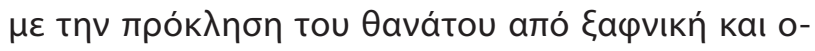

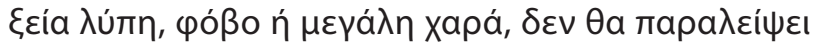

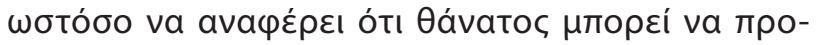

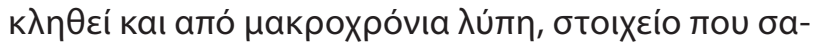

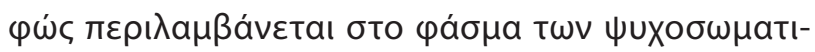

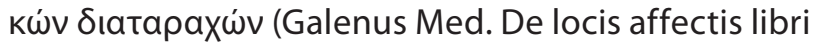
vi 8.301.14-8.302.5). ${ }^{24}$

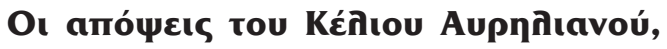

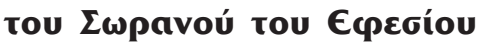

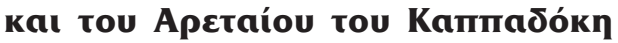

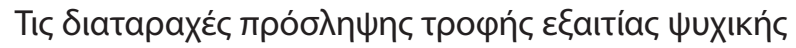

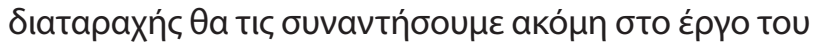
Caelius Aurelianus (5oc $\mu$.X. aı.) (Caelius Aurelianus On

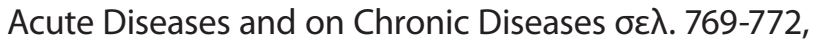

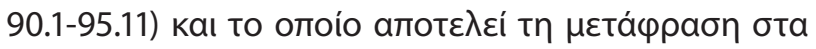

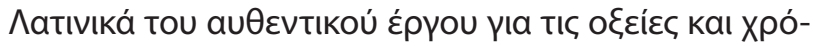

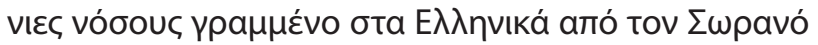

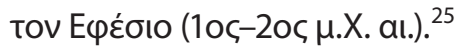

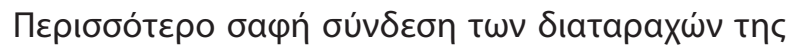

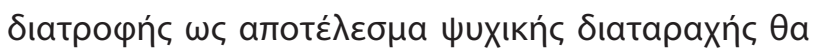

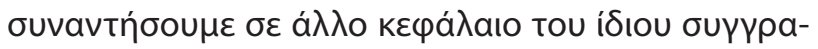

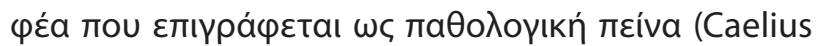
Aurelianus On Acute Diseases and on Chronic Diseases

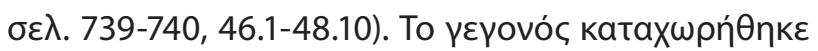

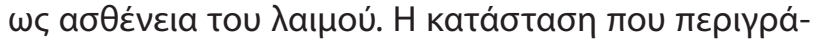

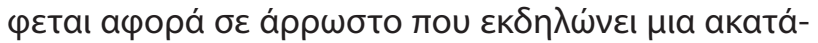

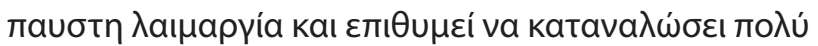

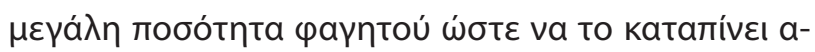

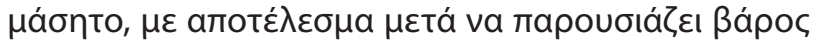

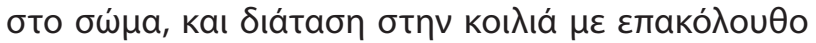

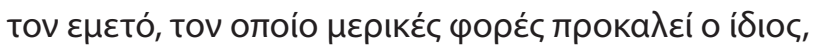

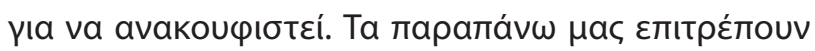

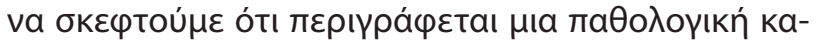

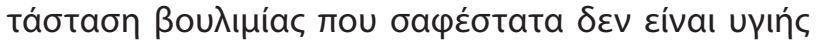

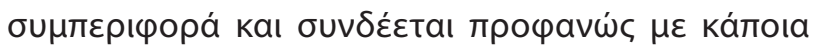

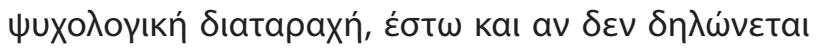

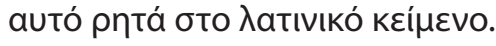

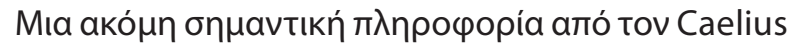

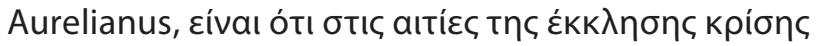

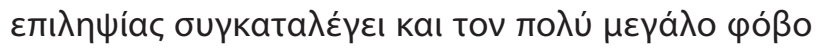

(Caelius Aurelianus On Acute Diseases and on Chronic

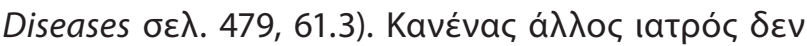

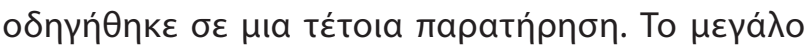

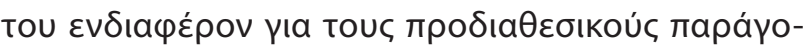

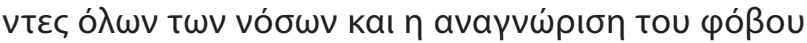

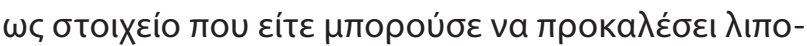

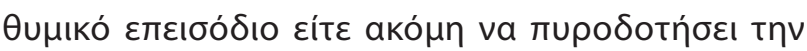

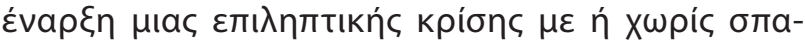

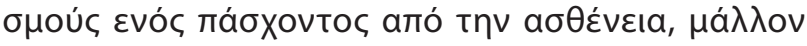

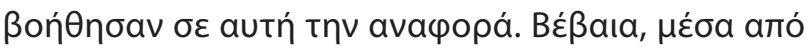

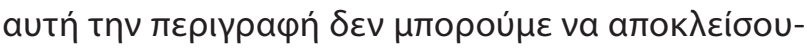

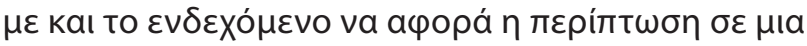
крíon votepíac.

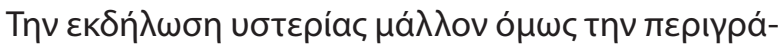

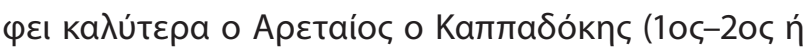

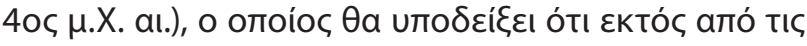

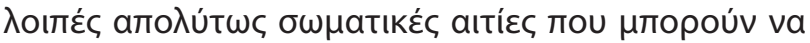

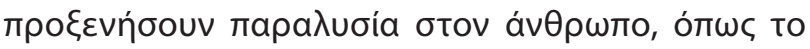

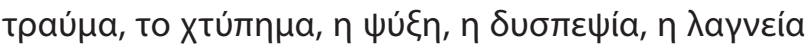

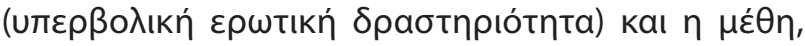

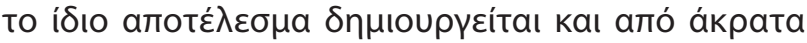

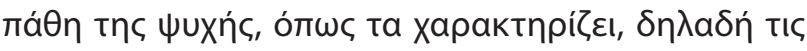

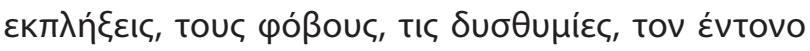

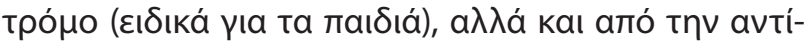

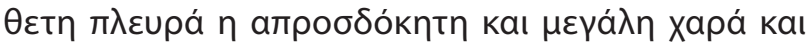

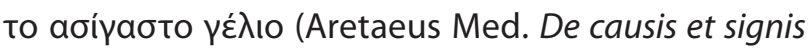
acutorum morborum 1.7.8.1-4). ${ }^{26}$

\section{Emínoyos}

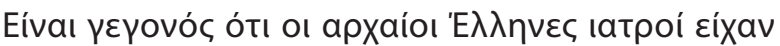

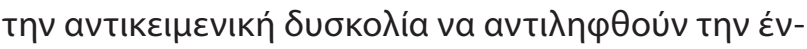

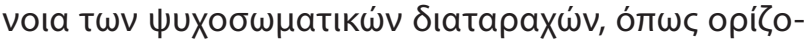

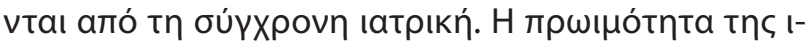

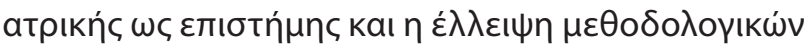

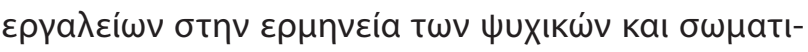

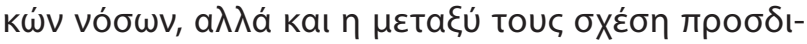

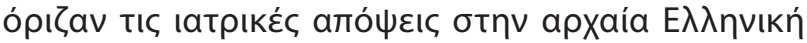

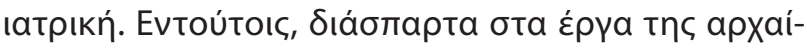

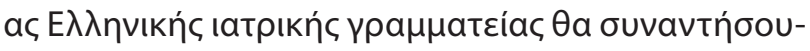

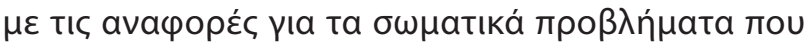

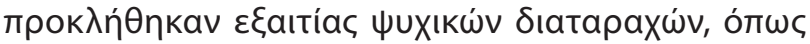

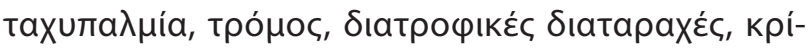

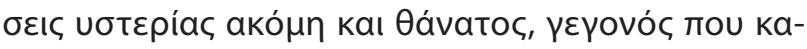

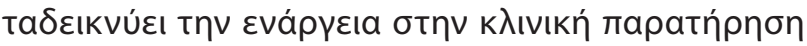

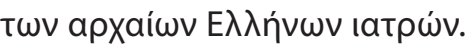




\title{
Psychosomatic disorders in ancient Greek medicine
}

\author{
K. Laios, ${ }^{1}$ M.-I. Kontaxaki, ${ }^{1}$ K. Markatos, ${ }^{1}$ E. Lagiou, ${ }^{2}$ \\ M. Karamanou, ${ }^{3,4}$ G. Androutsos ${ }^{4}$ \\ ${ }^{1}$ Department of History in Medicine, Medical School, University of Athens, Athens, \\ ${ }^{2}$ Medical School, University of Patra, Patra, \\ ${ }^{3}$ Department of History in Medicine, Medical School, University of Crete, Crete, \\ ${ }^{4}$ Biomedical Research Foundation, Academy of Athens, Athens, Greece
}

Psychiatriki 2018, 29:130-136

The concept of psychosomatic disorders, as defined by modern medicine, was difficult to be perceived by the ancient Greek physicians. Two main reasons contributed to this. One was that physicians in Greek antiquity had formed the idea that the mental illnesses that were recognized at that time, namely mania, melancholy, frenzy, caros, lethargy, apoplexy, but even epilepsy, was the result of a disturbance of the essential elements of the body, the balance of them contributed to the preservation of health. Thus, depending on the school of medical thought of each physician in antiquity, mental and corporal illnesses were the result of various disorders such as the dyscrasia of humors for the physicians of the Dogmatic school that followed the Hippocratic principles or the disorder of the qualitative characteristics of the humor and the pneuma (air), as the physicians of the Pneumatic School considered, but also of the stenosis or the expansion of the pores as the physicians of the Methodic school thought. Although there was the perception that the diseases were the result of various combinations of the previous theories, as concluded by the physicians who constituted the Eclectic school. The second reason was that ancient physicians could not perceive the autonomy of man's psychic world as an element of human nature in which emotional distress and irrational mental processing of stimuli from the social, cultural and natural environment of the individual would be aggravating to the challenge of mental imbalance. Nevertheless, many physicians such as physicians who wrote various work of Corpus Hippocraticum, Soranus of Ephesus (1st - 2nd c. AD) Galen (1st - 2nd c. AD), Aretaeus of Cappadocia (1st - 2nd or 4th c. AD) and Caelius Aurelianus (5th c. AD) did not forget to describe in their works psychosomatic disorders as they are defined by modern medicine. In their works there are the observations about intense sweating, tremor, eating disorders, hysteria and even death as a result of an intense and long psychological unrest. These corporal symptoms, although were onset due to a psychological unequilibrium they could not been listed by the ancient Greek physicians in any of the mental diseases as they were defined in antiquity. The psychological disturbance which could provoke the above corporal disorders arose by various phobias, shame, sorrow, anger, envy, excessive drinks and food, excessive sexual desire, passion for gambling and anxiety of everyday life.

Key words: Psychosomatic disorders, ancient Greek medicine, Galen, Corpus Hippocraticum.

\section{BıBntoypacpía}

1. Pigeaud J. Folie et cures de la folie chez les medecins de l'antiquite greco-romaine. La manie. Les belles lettres, Paris, 2010

2. Flashar H. Melancholie und melancholiker in der medizinischen theorien in der antike. Gruyter, Berlin, 1966
3. McDonald G. Concepts and Treatments of Phrenitis in Ancient Medicine. Ph.D. Thessis. Newcastle University, 2009

4. Laios K. Mental Diseases according to ancient Greek Physician. Post-Doc Study. Medical School. National and Kapodistrian University of Athens, 2013 
5. Simon B. Mind and Madness in Ancient Greece. The Classical Roots of Modern Psychiatry. Cornell University Press, Ithaca NY, 1978

6. Laios K, Giatsiou St, Tsoucalas Gr, Makaritsis K, Daios G, Sgantzos M. Apoplexy inside Hippocratic Collection. Iatriki 2015, 104:288-292

7. Temkin O. The Falling Sickness. History of Epilepsy from the Greeks to the Beginnings of Modern Neurology. Johns Hopkins University Press, Baltimore, London, 1994

8. Schöner E. Das viererschema in der antiken humoralpathologie. Steiner, Wiesbaden, 1964

9. Prioreschi P. A History of Medicine. Greek Medicine. II. Horatius Press, Omaha, 1994

10. Leven KH. Antike Medizine: Ein Lexikon. C.H. Beck, München, 2005

11. Tecusan M. The Fragments of the Methodists: Methodism Outside Soranus. Brill, Leiden, Boston, 2004

12. Nutton V. Ancient Medicine. Routledge, London, New York, 2004

13. Pigeaud J. La maladie de l'ame. Itude sur la relation de l'ame et $d u$ corps dans la tradition medico-philosophique antique. Les belles lettres, Paris, 2006

14. Chaignet A (ed) Histoire de la psychologie des Grecs, I-V. Paris, 1966re

15. Littré I (ed) De humoribus. Oeuvres complètes d'Hippocrate, vol. 5. Baillière, Paris, 1846

16. Kühn CG (ed) Ars medica. Claudii Galeni opera omnia, vol. 1. Knobloch, Leipzig, 1821

17. Kóhn CG (ed) De methodo medendi libri xiv. Claudii Galeni opera omnia. vol. 10. Knobloch, Leipzig, 1825

18. Koch C (ed) De sanitate tuenda libri vi. Leipzig, Teubner, 1923

19. Kühn CG (ed) De symptomatum causis libri iii. Claudii Galeni opera omnia. vol. 7. Knobloch, Leipzig, 1824
20. Alexanderson B (ed) De crisibus libri iii. Galenos. Studia Graeca et Latina Gothoburgensia 23. Elanders, Göteborg, 1967

21. Kühn CG (ed) De pulsibus libellus ad tirones. Claudii Galeni opera omnia. vol. 8. Knobloch, Leipzig, 1824

22. Kühn CG (ed) De tremore, palpitatione, convulsione et rigore liber. Claudii Galeni opera omnia. vol. 7. Knobloch, Leipzig, 1824

23. Kóhn CG (ed) De humero iis modis prolapso quos Hippocrates non vidit. Claudii Galeni opera omnia. vol. 18.1. Knobloch, Leipzig, 1829

24. Kóhn CG (ed) De locis affectis libri vi. Claudii Galeni opera omnia. vol. 8. Knobloch, Leipzig, 1824

25. Drabkin IE (ed and Trans.) Caelius Aurelianus. On Acute Diseases and On Chronic Diseases. University of Chicago Press, Chicago, 1950

26. Hude K (ed) Aretaeus. Corpus medicorum Graecorum, vol. 2. Akademie-Verlag, Berlin, 1958

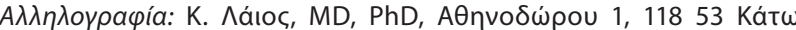

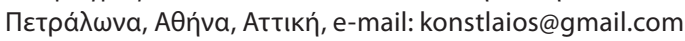




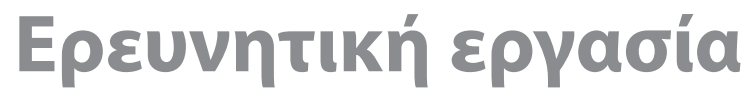 Research article
}

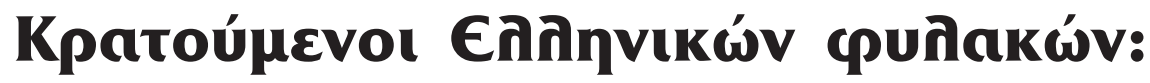

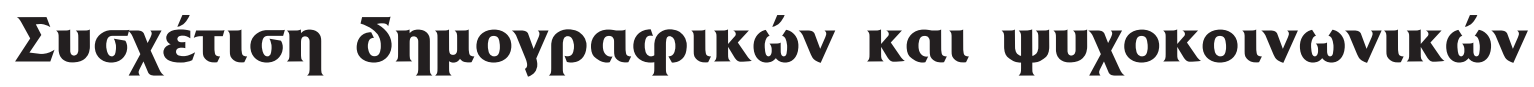

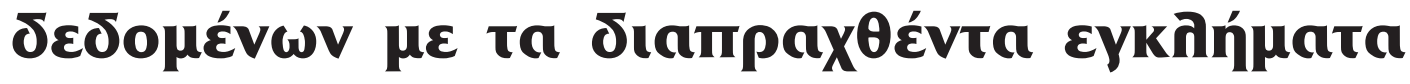

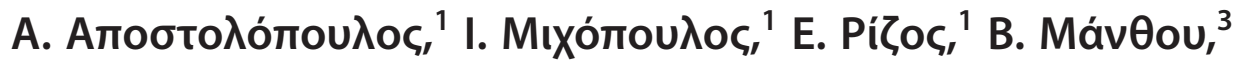

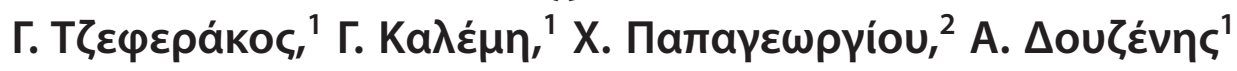

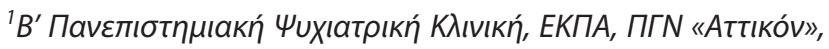

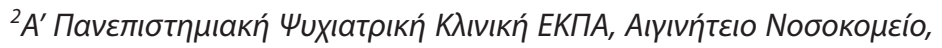

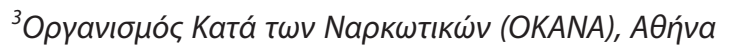

Uuxıatpıkń 2018, 29:137-148

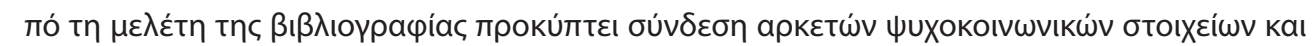

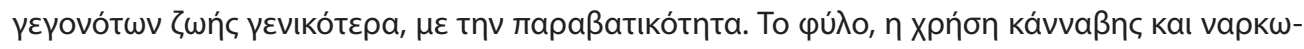

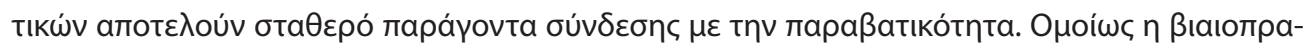

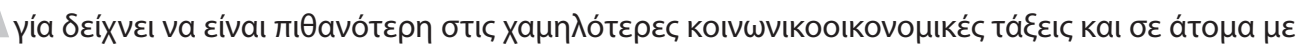

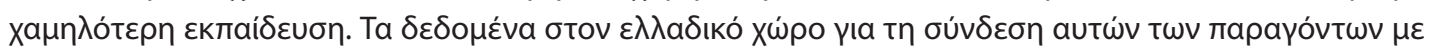

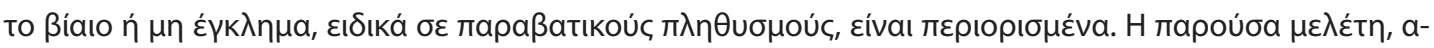

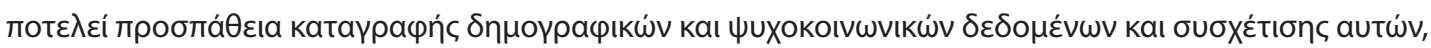

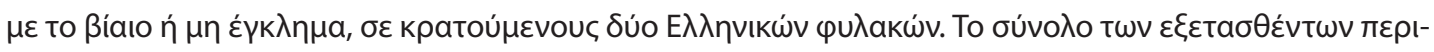

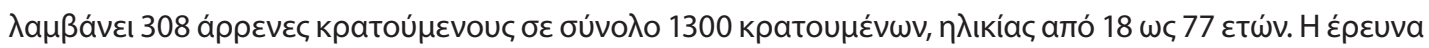

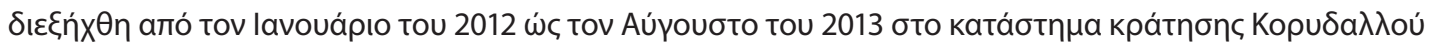

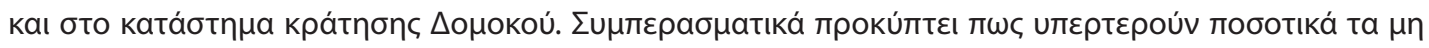

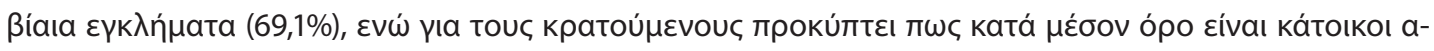

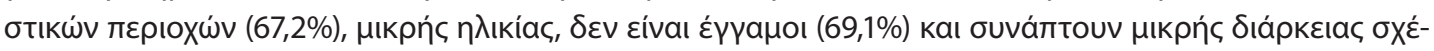

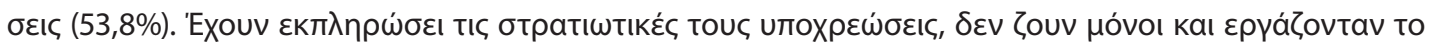

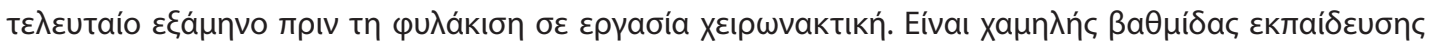

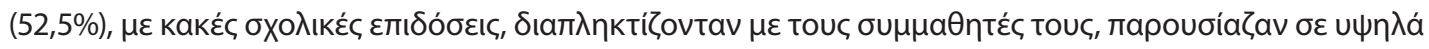

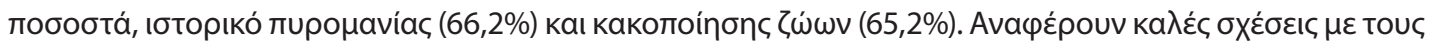

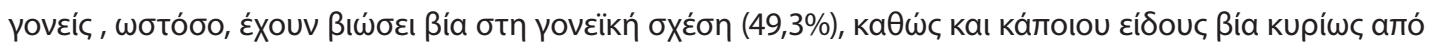

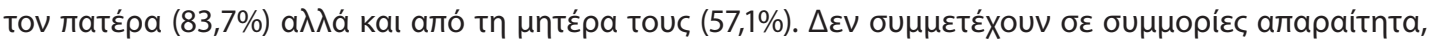




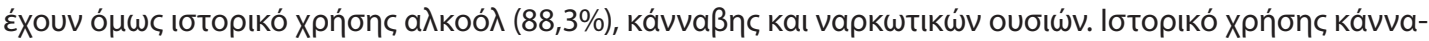

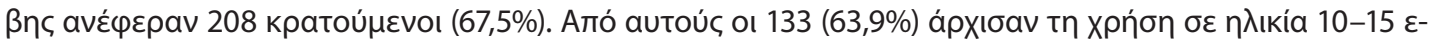

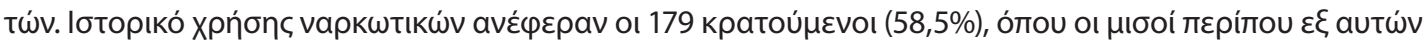

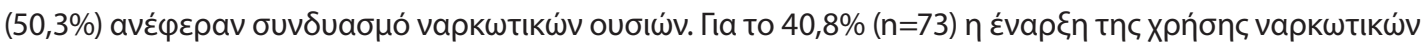

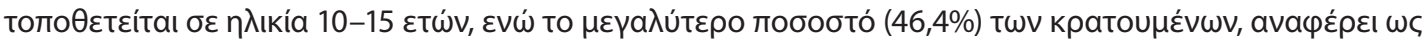

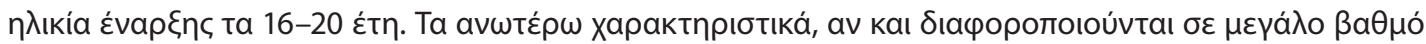

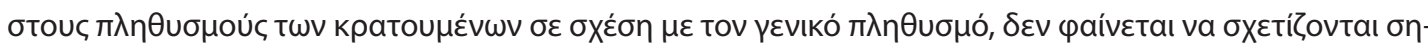

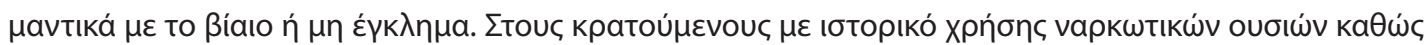

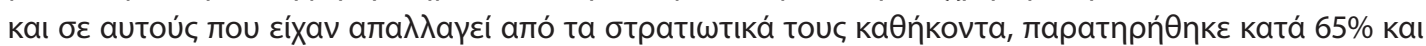

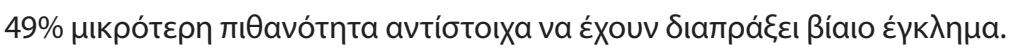

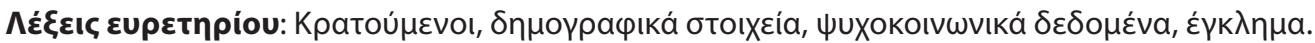

\section{Eıбaywyń}

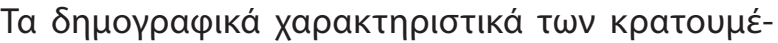

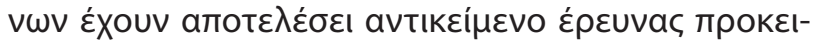

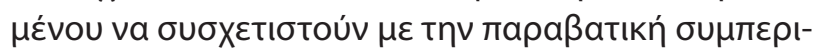

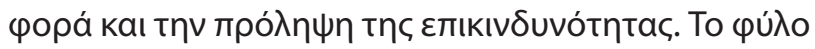

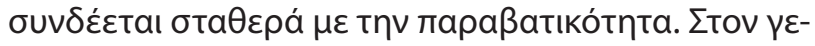

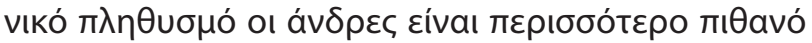

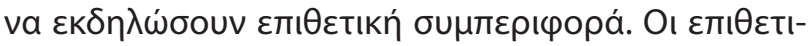

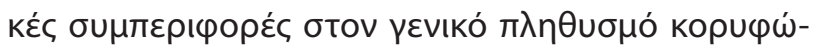

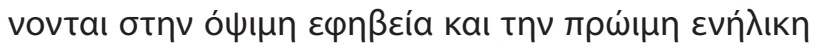

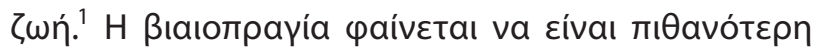

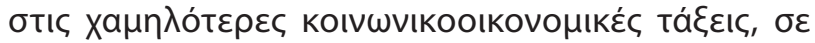

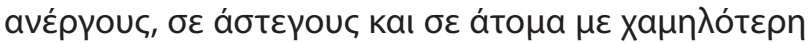
$\varepsilon \kappa \pi a i ́ \delta \varepsilon \cup \sigma \eta{ }^{2-4}$

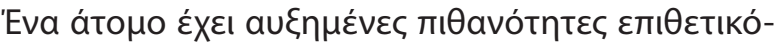

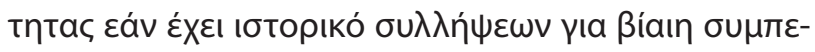

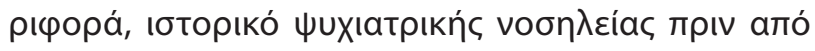

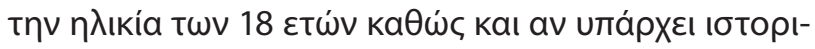

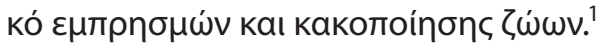

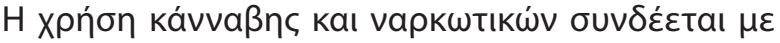

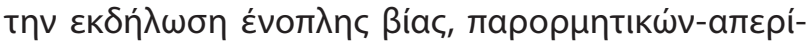

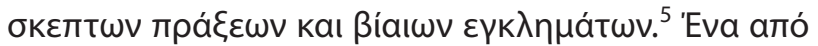

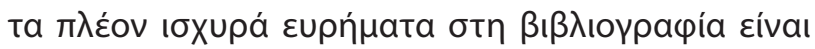

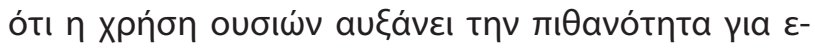

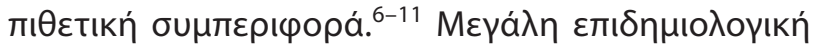

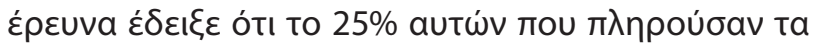

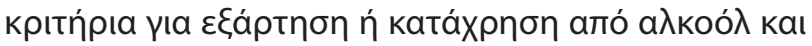

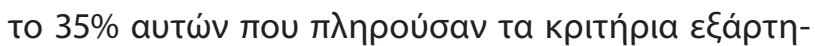

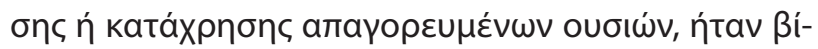

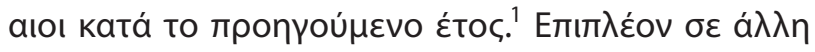

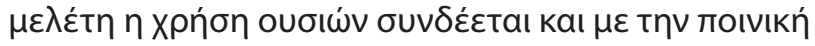

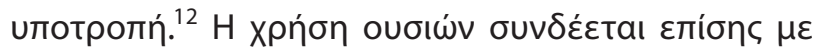

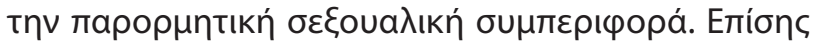

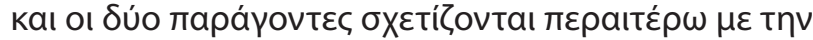
параßатıко́тпта. ${ }^{13-14}$

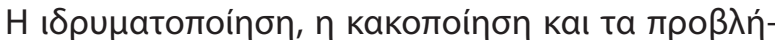

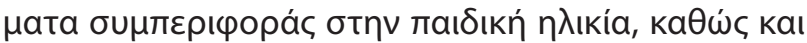

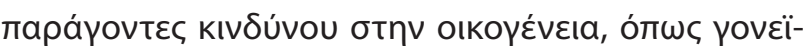

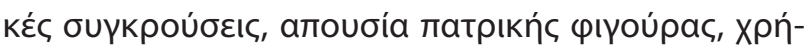

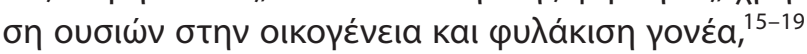

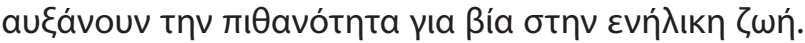

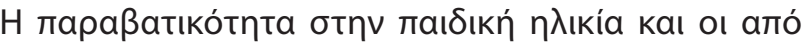

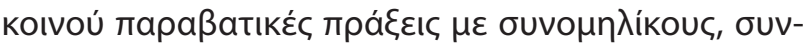

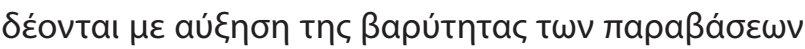

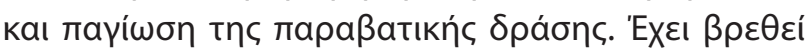

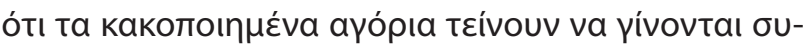

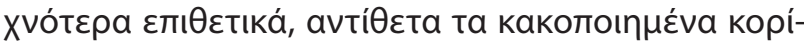

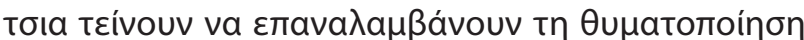

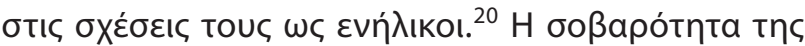

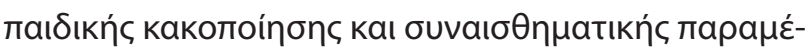

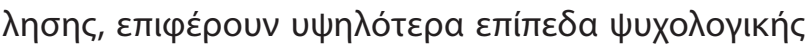

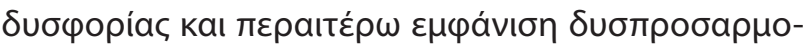

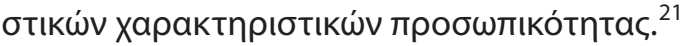

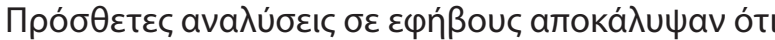

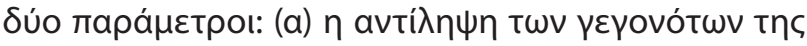

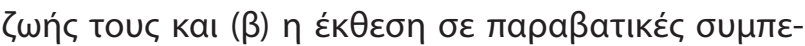

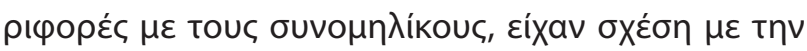

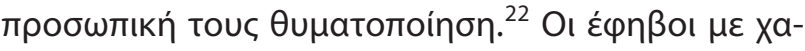

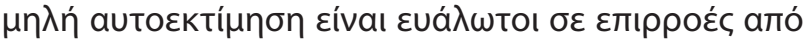

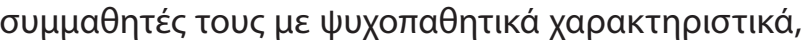

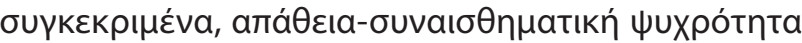

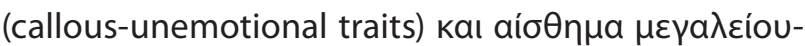

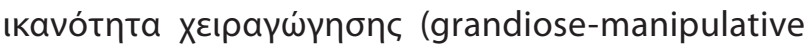

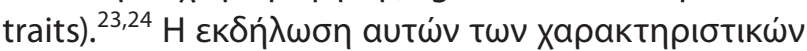

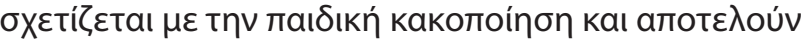

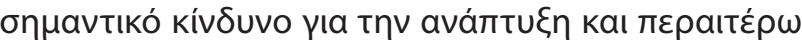

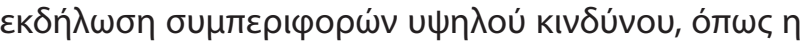




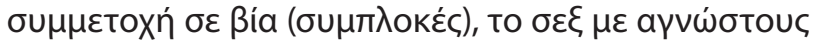

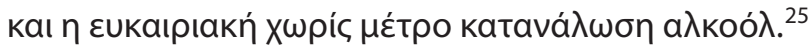

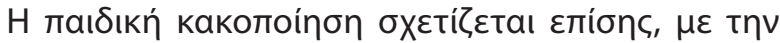

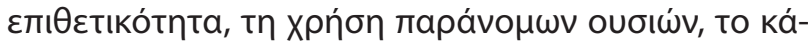

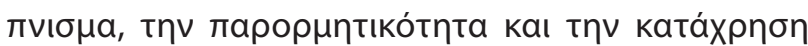

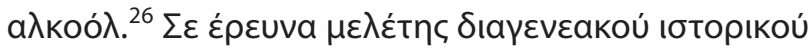

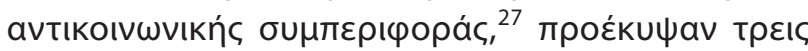

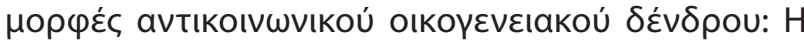

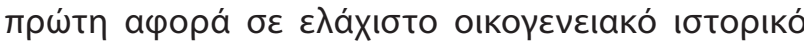

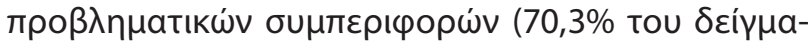

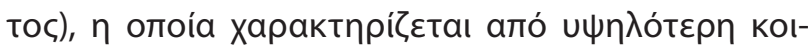

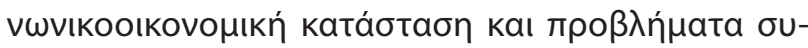

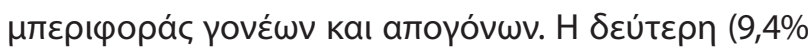

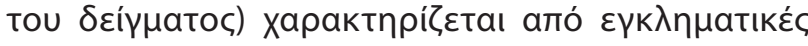

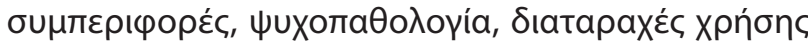

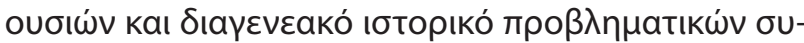

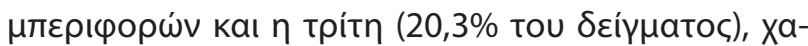

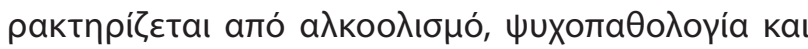

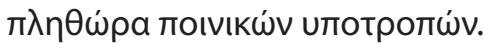

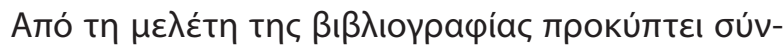

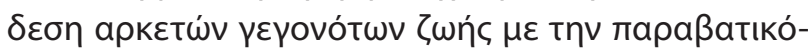

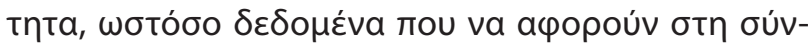

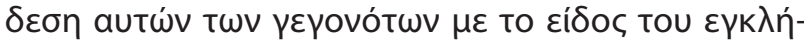

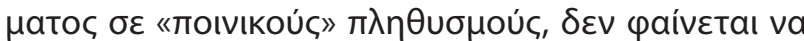

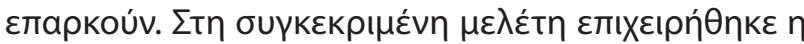

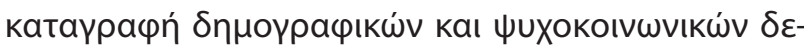

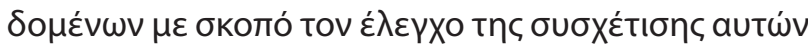

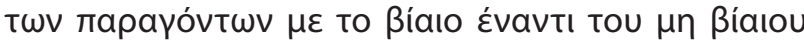

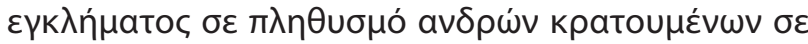

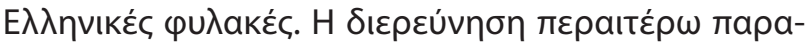

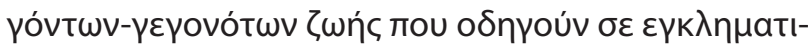

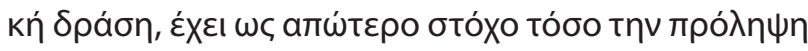

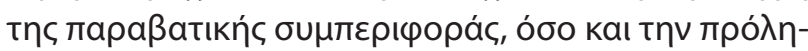

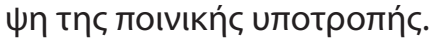

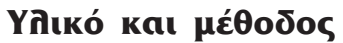

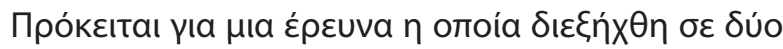

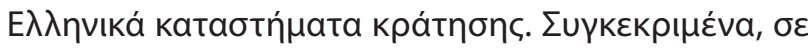

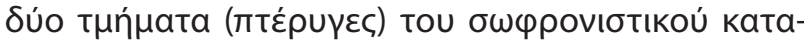

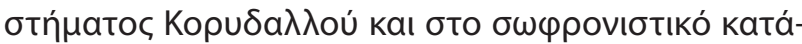

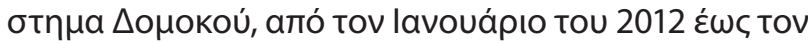

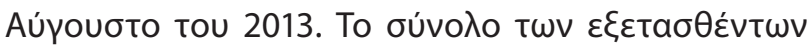

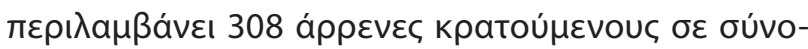

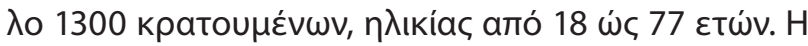

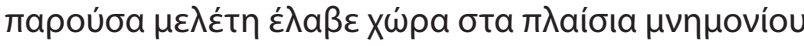

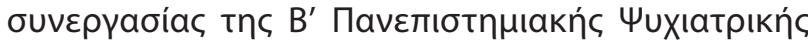

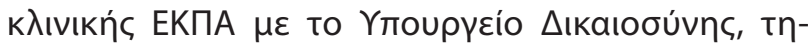

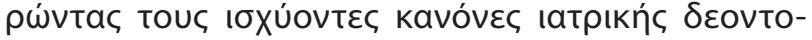

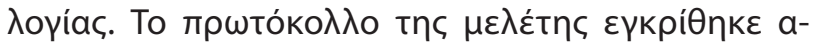

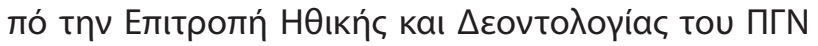

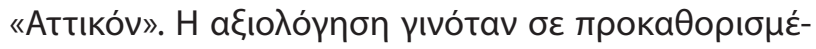

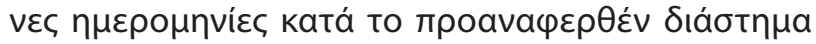

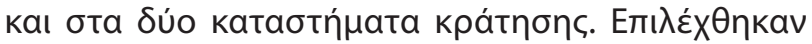

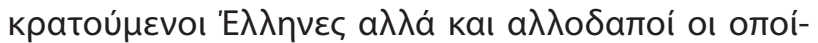

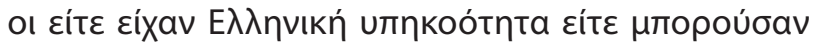

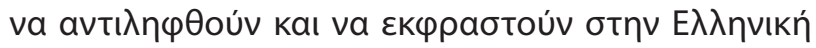

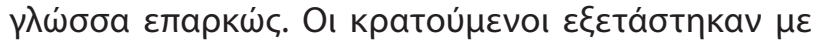

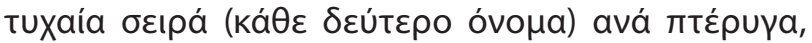

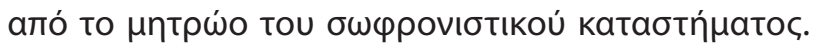

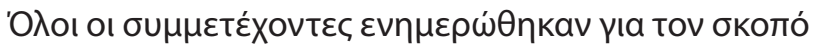

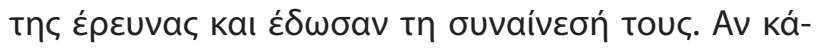

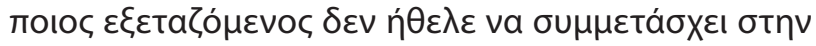

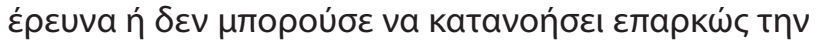

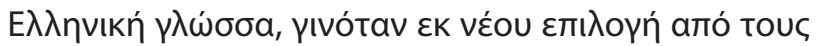

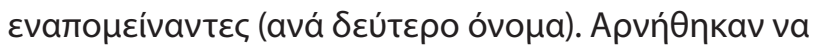

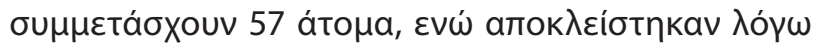

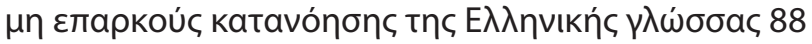

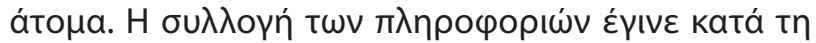

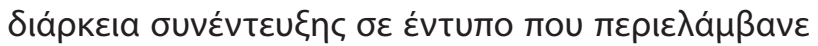

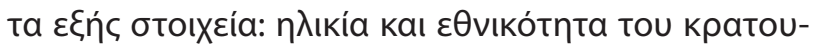

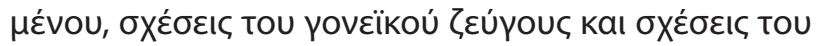

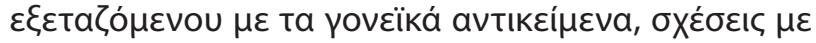

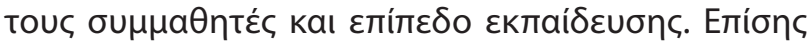

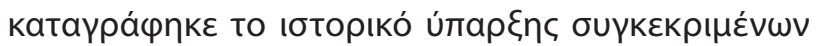

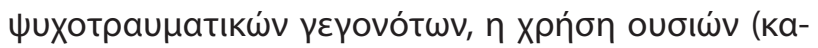

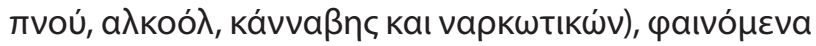

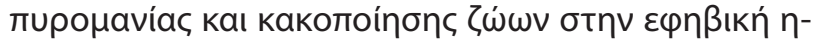

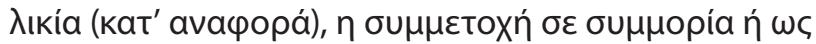

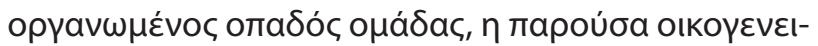

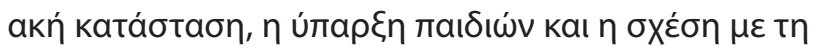

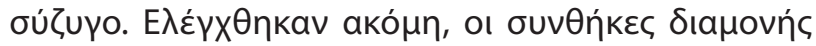

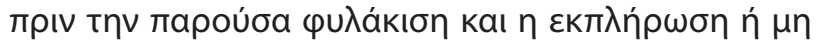

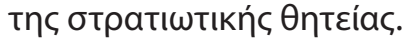

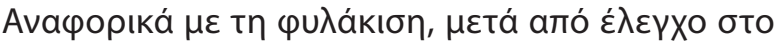

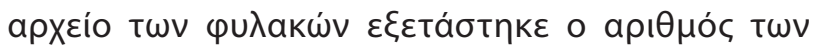

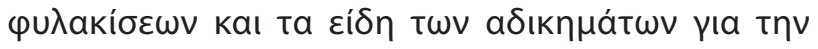

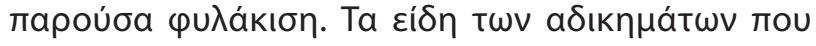

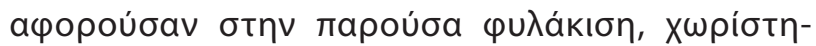

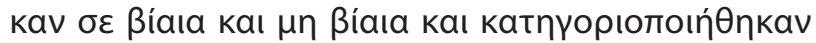

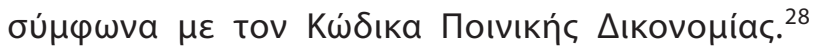

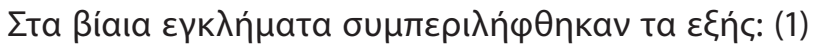

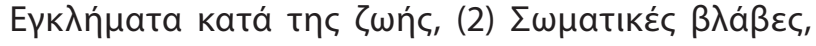

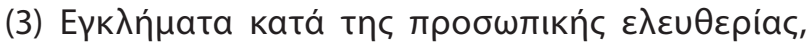

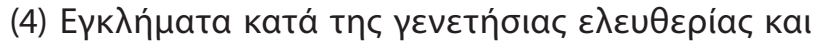




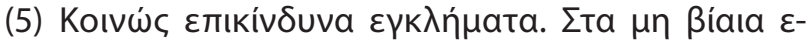

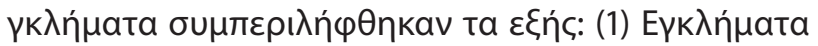

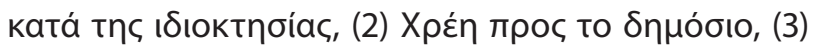

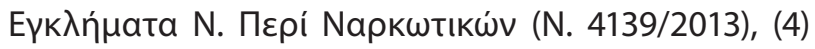

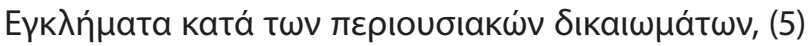

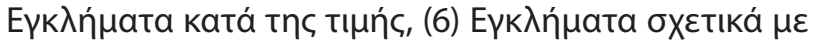

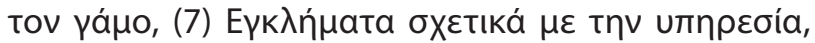

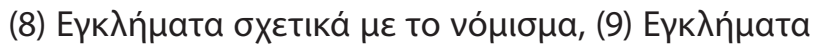

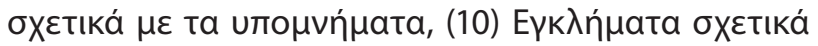

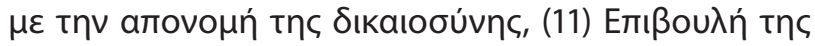

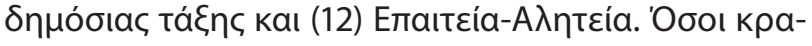

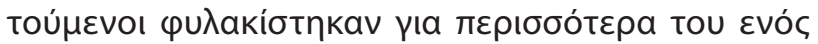

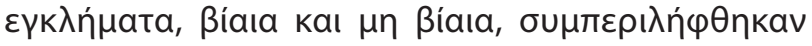

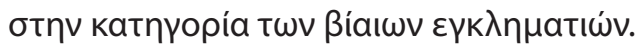

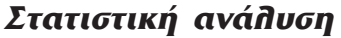

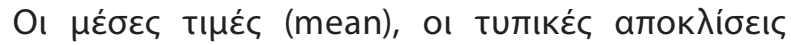

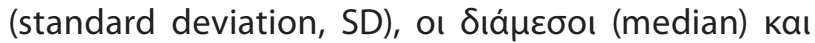

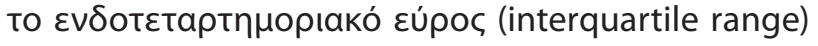

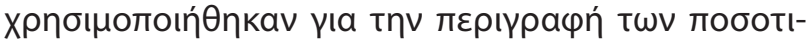

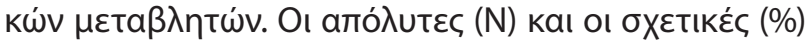

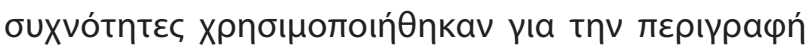

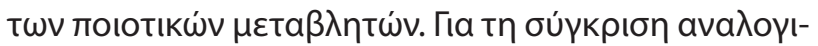

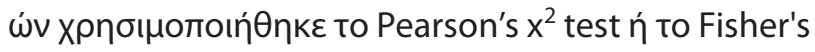

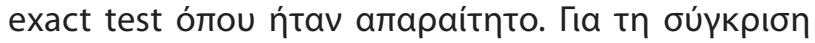

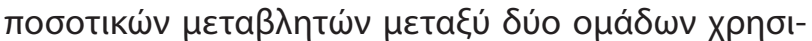

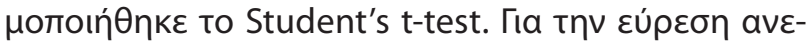

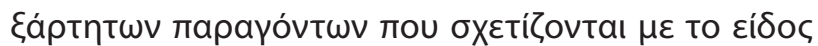

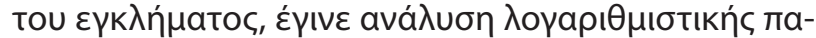
$\lambda ı v \delta \rho o ́ \mu \eta \sigma n c$ (logistic regression analysis) $\mu \varepsilon$ in $\delta ı$ -

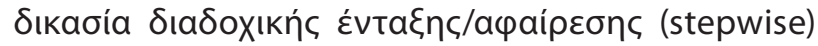

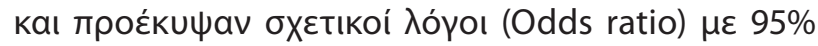

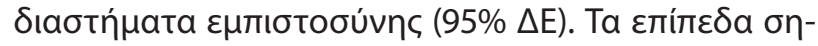

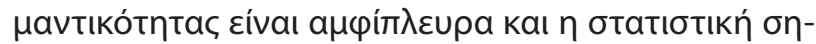

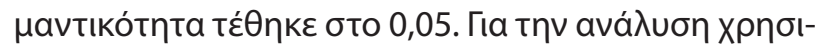

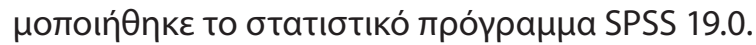

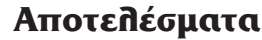

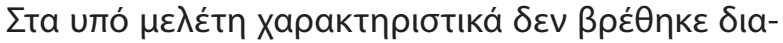

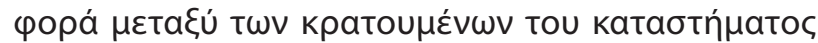

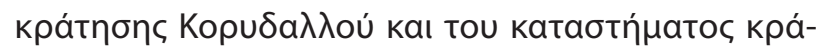

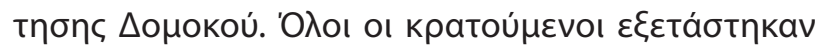

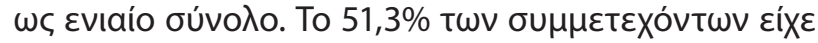

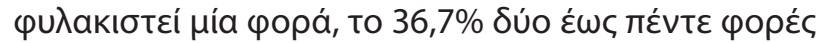

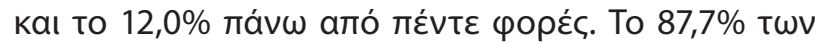

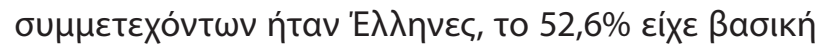

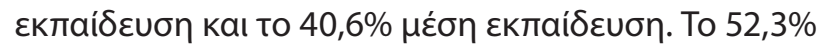

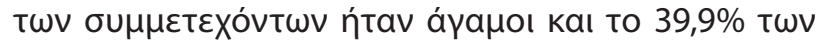

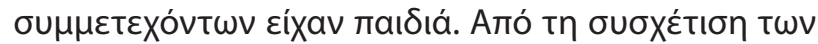

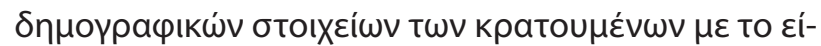

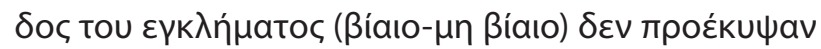

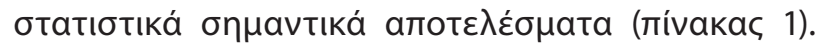

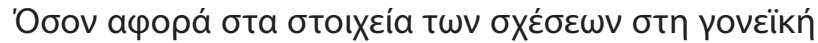

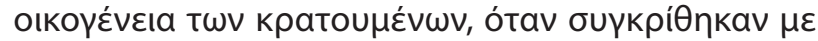

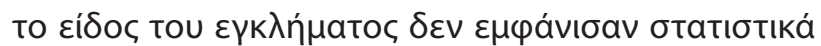

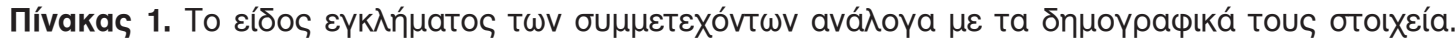

\begin{tabular}{|c|c|c|c|c|c|c|}
\hline & & \multicolumn{4}{|c|}{ 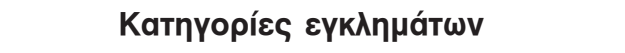 } & \multirow{3}{*}{$\begin{array}{l}\text { p Pearson's } \\
x^{2} \text { test }\end{array}$} \\
\hline & & \multicolumn{2}{|c|}{ Mn ßíaıa } & \multicolumn{2}{|c|}{ Bíaıa/ Kaı тa dúo } & \\
\hline & & $n$ & (\%) & $n$ & (\%) & \\
\hline \multicolumn{2}{|c|}{ H入ıкía (MO, TA) } & \multicolumn{2}{|c|}{$37,9(10,9)$} & \multicolumn{2}{|c|}{$39,2(10,6)$} & $0,339 *$ \\
\hline \multirow[t]{2}{*}{ ЕӨvıкóтпта } & 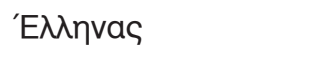 & 190 & 70,4 & 80 & 29,6 & \multirow{2}{*}{0,219} \\
\hline & 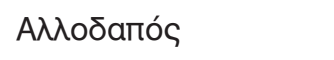 & 23 & 60,5 & 15 & 39,5 & \\
\hline \multirow[t]{2}{*}{ 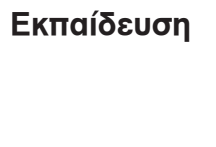 } & Вабıкń & 112 & 69,1 & 50 & 30,9 & \multirow[b]{2}{*}{0,994} \\
\hline & 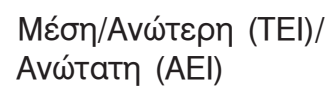 & 101 & 69,2 & 45 & 30,8 & \\
\hline \multirow[t]{2}{*}{ 'Еүүано } & 'Oxı & 141 & 66,2 & 72 & 33,8 & \multirow{2}{*}{0,077} \\
\hline & Nal & 71 & 76,3 & 22 & 23,7 & \\
\hline \multirow[t]{2}{*}{ Tékva } & Nal & 85 & 69,1 & 38 & 30,9 & \multirow{2}{*}{0,988} \\
\hline & 'OXl & 128 & 69,2 & 57 & 30,8 & \\
\hline
\end{tabular}

*Student's t-test 


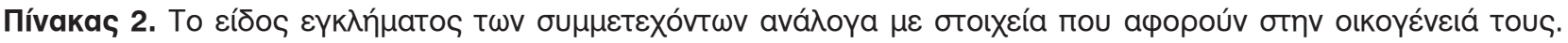

\begin{tabular}{|c|c|c|c|c|c|c|}
\hline & & \multicolumn{4}{|c|}{ 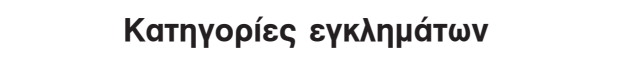 } & \multirow{3}{*}{$\begin{array}{c}p \\
\text { Pearson's } \\
x^{2} \text { test }\end{array}$} \\
\hline & & \multicolumn{2}{|c|}{ Mn Bíaıa } & \multicolumn{2}{|c|}{ Bíaıa/ Kaı тa dúo } & \\
\hline & & $\mathbf{n}$ & (\%) & $\mathbf{n}$ & (\%) & \\
\hline \multirow{4}{*}{ 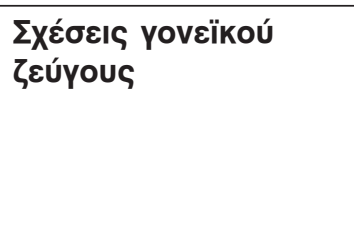 } & Ka入źৎ & 126 & 67,4 & 61 & 32,6 & \multirow{4}{*}{0,112} \\
\hline & Какह́ৎ & 46 & 75,4 & 15 & 24,6 & \\
\hline & Аঠıáфорєৎ & 23 & 59,0 & 16 & 41,0 & \\
\hline & 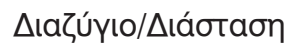 & 18 & 85,7 & 3 & 14,3 & \\
\hline \multirow{2}{*}{ 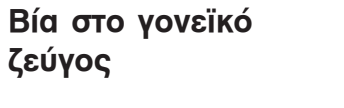 } & 'Oxı & 109 & 69,9 & 47 & 30,1 & \multirow{2}{*}{0,783} \\
\hline & Nal & 104 & 68,4 & 48 & 31,6 & \\
\hline \multirow[t]{3}{*}{ 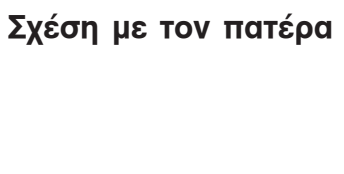 } & Ka入ń & 133 & 69,3 & 59 & 30,7 & \multirow{3}{*}{0,360} \\
\hline & Каки́ & 47 & 74,6 & 16 & 25,4 & \\
\hline & 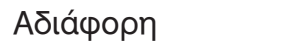 & 33 & 62,3 & 20 & 37,7 & \\
\hline \multirow[t]{2}{*}{ Bía aпó tov пате́pa } & 'Oxı & 31 & 67,4 & 15 & 32,6 & \multirow{2}{*}{0,788} \\
\hline & Nal & 179 & 69,4 & 79 & 30,6 & \\
\hline \multirow[t]{3}{*}{ 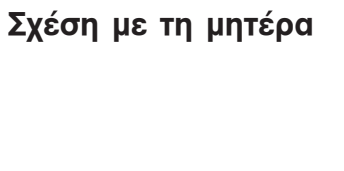 } & Kaגń & 169 & 69,0 & 76 & 31,0 & \multirow{3}{*}{0,115} \\
\hline & Какń & 13 & 92,9 & 1 & 7,1 & \\
\hline & 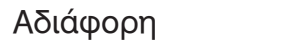 & 30 & 63,8 & 17 & 36,2 & \\
\hline \multirow[t]{2}{*}{ 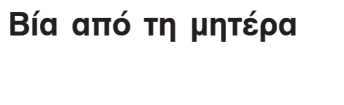 } & 'Oxı & 85 & 68,0 & 40 & 32,0 & \multirow{2}{*}{0,727} \\
\hline & Nal & 123 & 69,9 & 53 & 30,1 & \\
\hline
\end{tabular}

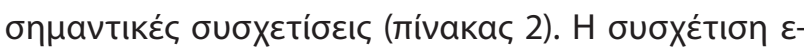

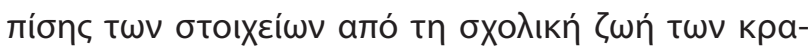

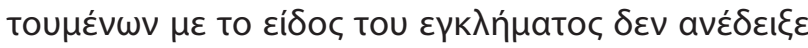

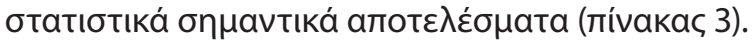

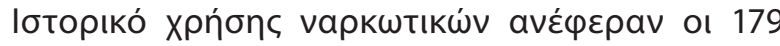

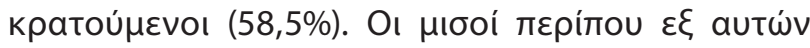

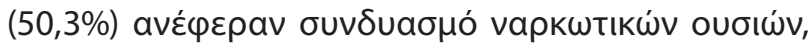

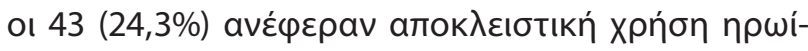

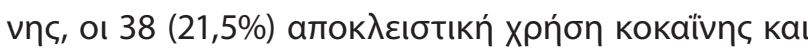

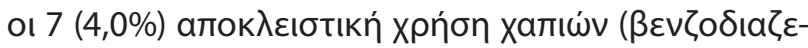

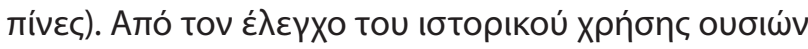

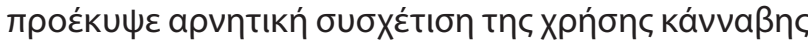

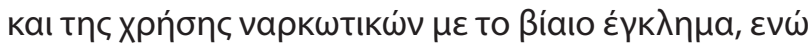

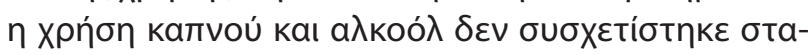

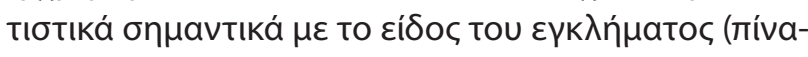
кас 4).

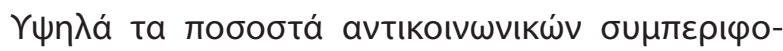

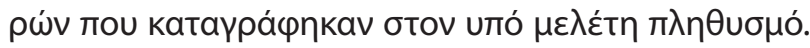

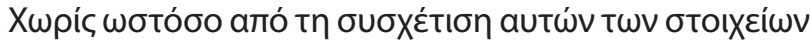

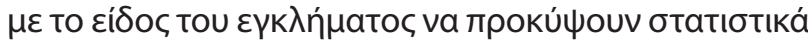

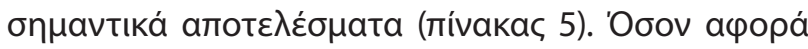

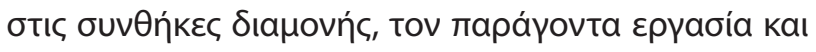

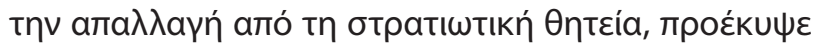

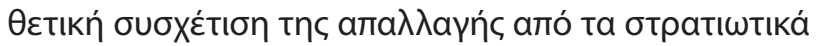

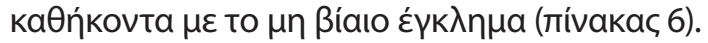

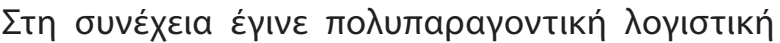

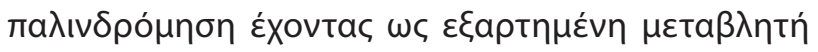

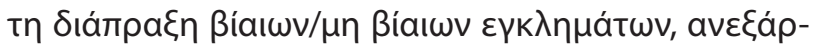

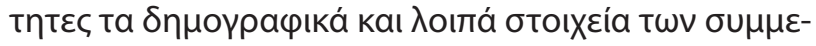

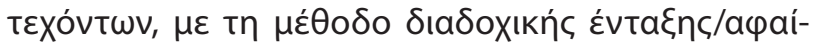

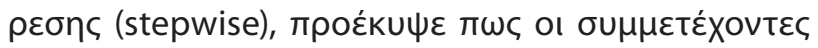

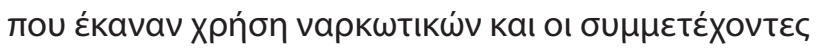

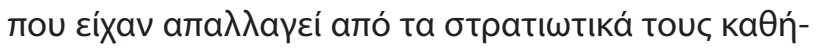

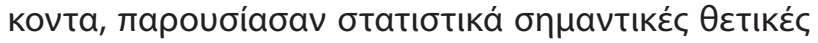

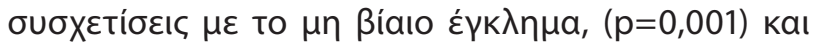
$(p=0,029)$ avtíotoıxa (лі́vaкac 7).

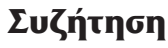

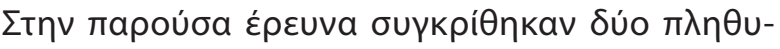

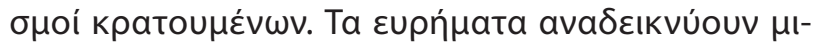

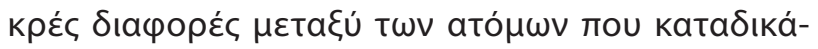




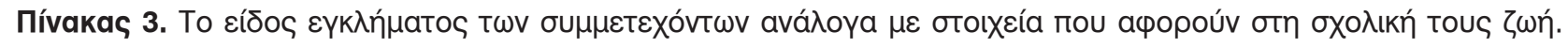

\begin{tabular}{|c|c|c|c|c|c|c|}
\hline & & \multicolumn{4}{|c|}{ 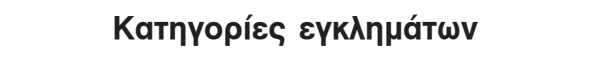 } & \multirow{3}{*}{$\begin{array}{l}\text { p Pearson's } \\
x^{2} \text { test }\end{array}$} \\
\hline & & \multicolumn{2}{|c|}{ Mn ßíaıa } & \multicolumn{2}{|c|}{ Bíaıa/Kaı тa ঠúo } & \\
\hline & & $\mathbf{n}$ & (\%) & $\mathbf{n}$ & (\%) & \\
\hline \multirow[t]{2}{*}{ 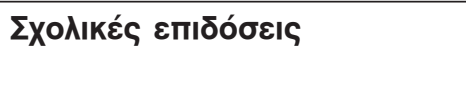 } & 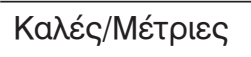 & 90 & 69,2 & 40 & 30,8 & \multirow{2}{*}{0,981} \\
\hline & Каке́ৎ & 123 & 69,1 & 55 & 30,9 & \\
\hline \multirow[t]{3}{*}{ 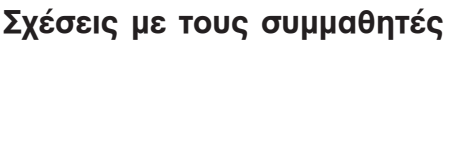 } & Ka入ćৎ & 69 & 68,3 & 32 & 31,7 & \multirow{3}{*}{0,358} \\
\hline & Какع́ৎ & 33 & 78,6 & 9 & 21,4 & \\
\hline & $\Delta$ เаплпктıбноі́ & 111 & 67,3 & 54 & 32,7 & \\
\hline \multirow[t]{2}{*}{ 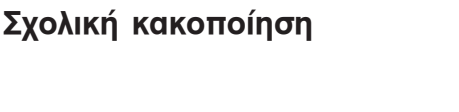 } & 'Oxı & 159 & 69,1 & 71 & 30,9 & \multirow{2}{*}{0,987} \\
\hline & Nal & 54 & 69,2 & 24 & 30,8 & \\
\hline \multirow[t]{2}{*}{ 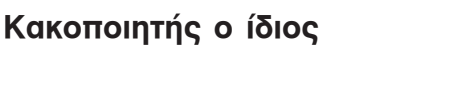 } & 'Oxı & 121 & 67,6 & 58 & 32,4 & \multirow{2}{*}{0,486} \\
\hline & Nal & 92 & 71,3 & 37 & 28,7 & \\
\hline
\end{tabular}

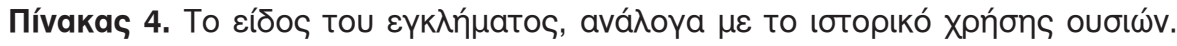

\begin{tabular}{|c|c|c|c|c|c|c|}
\hline & & \multicolumn{4}{|c|}{ 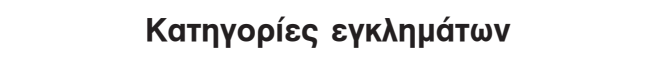 } & \multirow{3}{*}{$\begin{array}{c}p \text { Pearson's } x^{2} \\
\text { test }\end{array}$} \\
\hline & & \multicolumn{2}{|c|}{ Mn Bíaı́a } & \multicolumn{2}{|c|}{ Bíaıa/ Kaı ta ঠúo } & \\
\hline & & $\mathbf{n}$ & (\%) & $\mathbf{n}$ & (\%) & \\
\hline \multirow{2}{*}{ Xpńon kanvoú } & OXl & 10 & 76,9 & 3 & 23,1 & 0,761 \\
\hline & Nal & 203 & 68,8 & 92 & 31,2 & \\
\hline \multirow{2}{*}{ Хрп́бп аגкоо́ג } & Oxl & 26 & 72,2 & 10 & 27,8 & 0,672 \\
\hline & Nal & 187 & 68,8 & 85 & 31,3 & \\
\hline \multirow{2}{*}{ Xpńon kávvaßns } & 'Oxl & 58 & 58,0 & 42 & 42,0 & 0,003 \\
\hline & Nal & 155 & 74,5 & 53 & 25,5 & \\
\hline \multirow{2}{*}{ 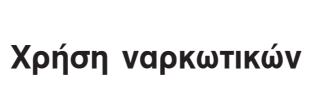 } & 'OXl & 71 & 55,9 & 56 & 44,1 & $<0,001$ \\
\hline & Nal & 141 & 78,8 & 38 & 21,2 & \\
\hline
\end{tabular}

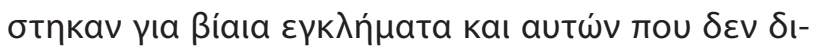

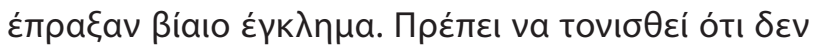

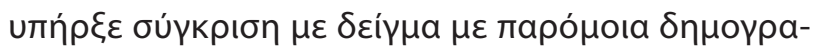

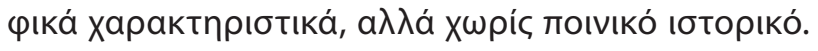

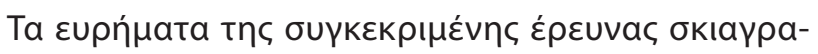

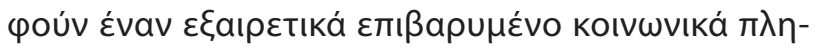

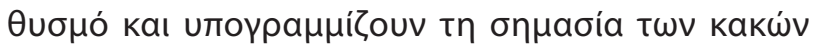

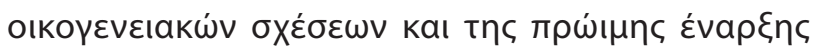

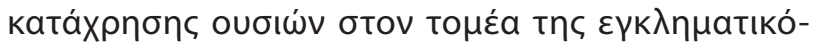
тптас.

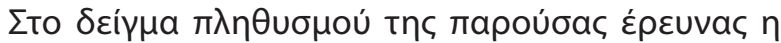

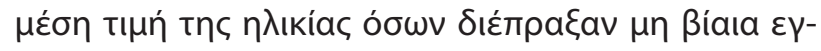

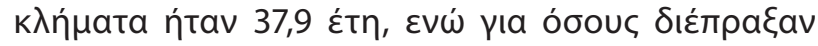

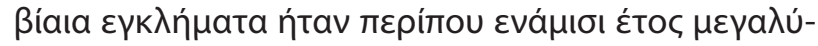

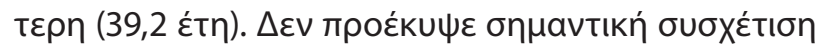

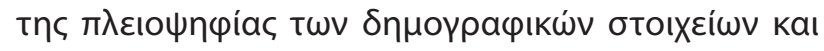

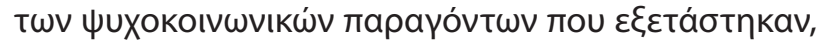
$\mu \varepsilon$ to ßíaı ń $\mu \eta$ ßíaı $\varepsilon$ $ү к \lambda \eta \mu a$.

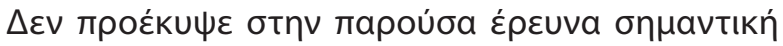

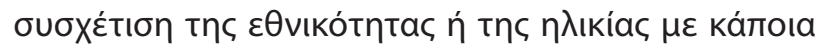




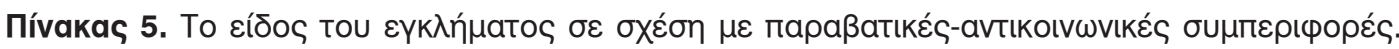

\begin{tabular}{|c|c|c|c|c|c|c|}
\hline & & \multicolumn{4}{|c|}{ 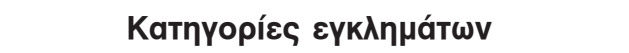 } & \multirow{3}{*}{$\begin{array}{l}\text { p Pearson's } \\
x^{2} \text { test }\end{array}$} \\
\hline & & \multicolumn{2}{|c|}{ Mn Bíaı́a } & \multicolumn{2}{|c|}{ Bíaıa/ Kaı ta dúo } & \\
\hline & & $\mathbf{n}$ & (\%) & $\mathbf{n}$ & (\%) & \\
\hline \multirow{2}{*}{ 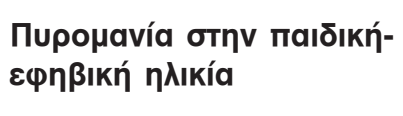 } & 'Oxl & 72 & 69,2 & 32 & 30,8 & \multirow{2}{*}{0,984} \\
\hline & Nal & 141 & 69,1 & 63 & 30,9 & \\
\hline \multirow{2}{*}{ 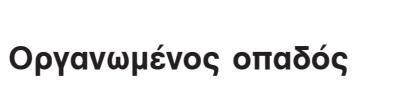 } & 'OXı & 180 & 69,2 & 80 & 30,8 & \multirow{2}{*}{0,947} \\
\hline & Nal & 33 & 68,8 & 15 & 31,3 & \\
\hline \multirow{2}{*}{ 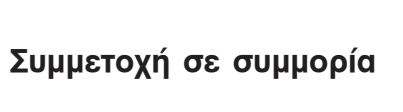 } & 'Oxl & 105 & 66,9 & 52 & 33,1 & \multirow{2}{*}{0,378} \\
\hline & Nal & 108 & 71,5 & 43 & 28,5 & \\
\hline \multirow{2}{*}{ 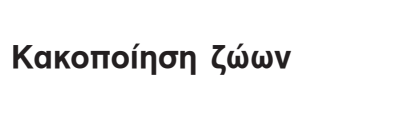 } & 'Oxı & 80 & 74,8 & 27 & 25,2 & \multirow{2}{*}{0,120} \\
\hline & Nal & 133 & 66,2 & 68 & 33,8 & \\
\hline \multirow{2}{*}{ 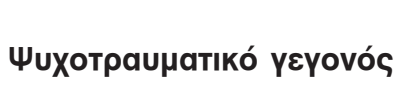 } & 'Oxl & 124 & 68,5 & 57 & 31,5 & \multirow{2}{*}{0,804} \\
\hline & Nal & 88 & 69,8 & 38 & 30,2 & \\
\hline \multirow{2}{*}{ 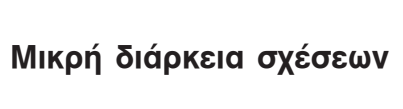 } & 'Oxı & 94 & 66,2 & 48 & 33,8 & \multirow{2}{*}{0,298} \\
\hline & Nal & 119 & 71,7 & 47 & 28,3 & \\
\hline \multirow{2}{*}{ 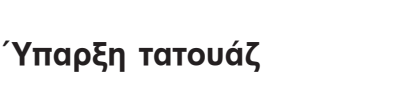 } & 'Oxı & 77 & 65,3 & 41 & 34,7 & \multirow{2}{*}{0,243} \\
\hline & Nal & 136 & 71,6 & 54 & 28,4 & \\
\hline
\end{tabular}

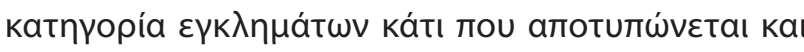

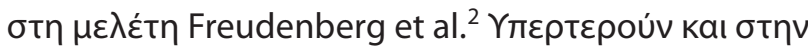

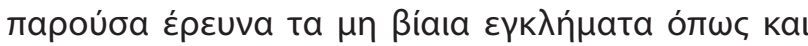

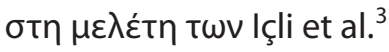

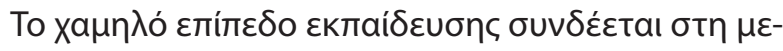

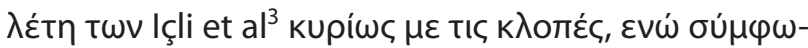

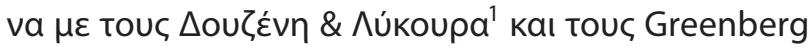

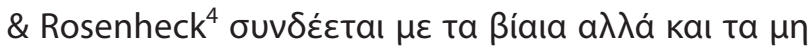

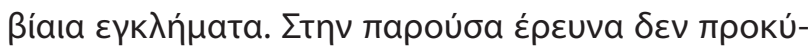

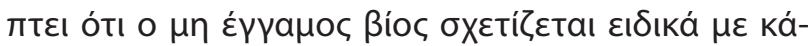

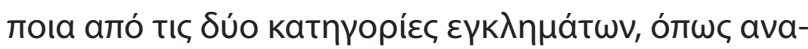

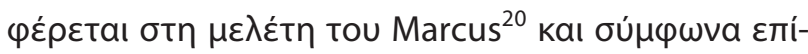

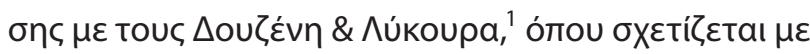

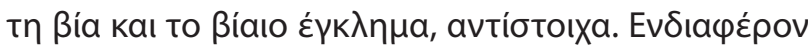

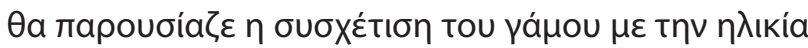

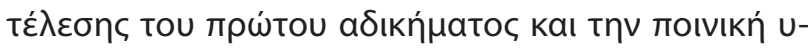

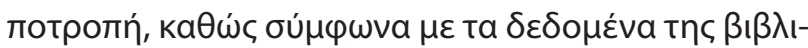

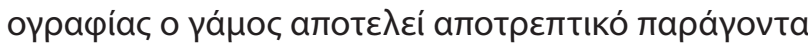
поІviкńc итотроти́c. ${ }^{29}$

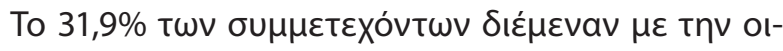

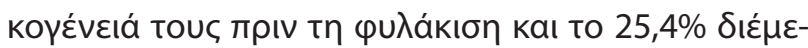

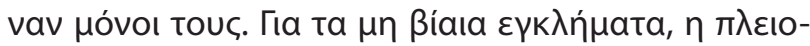

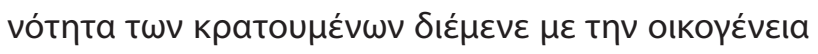

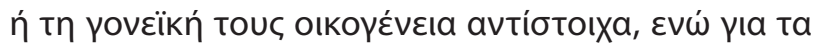
Bíala $\varepsilon ү k \lambda$ ń

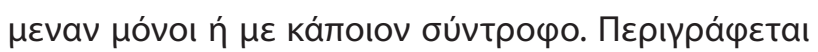

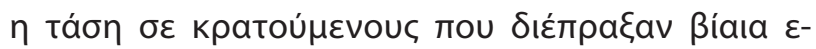

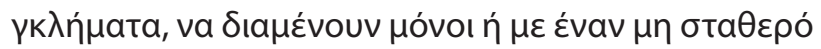

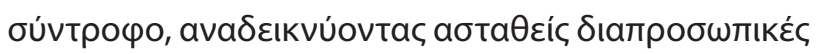

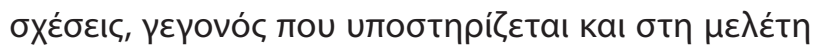
Tou Marcus. ${ }^{20}$

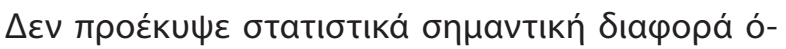

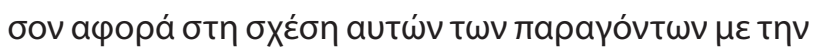

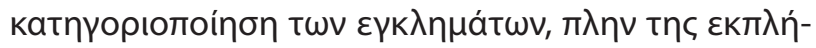

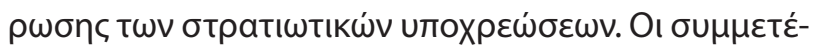

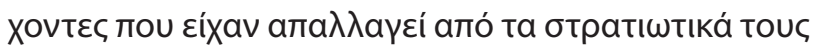

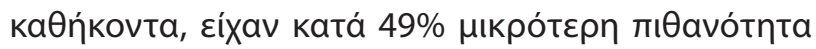

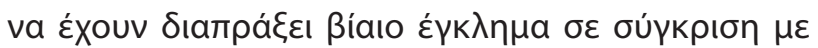

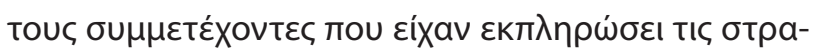

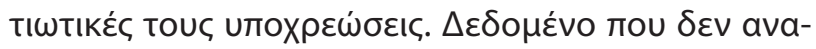

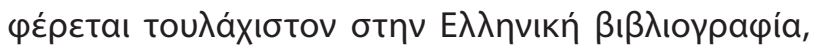

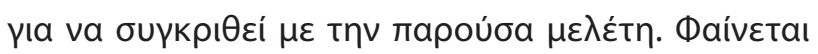

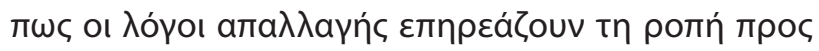

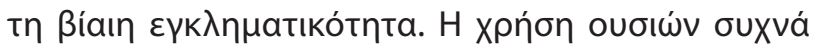




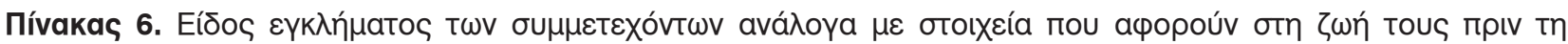

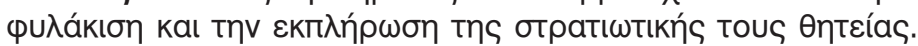

\begin{tabular}{|c|c|c|c|c|c|c|}
\hline & & \multicolumn{4}{|c|}{ 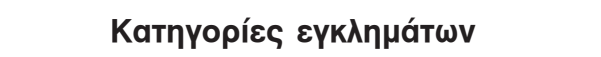 } & \multirow{3}{*}{$\begin{array}{c}p \\
\text { Pearson's } \\
x^{2} \text { test }\end{array}$} \\
\hline & & \multicolumn{2}{|c|}{ Mn Bíaıa } & \multicolumn{2}{|c|}{ Bíaıa/Kaı тa ঠúo } & \\
\hline & & $\mathrm{n}$ & (\%) & $\mathrm{n}$ & (\%) & \\
\hline \multirow{4}{*}{ 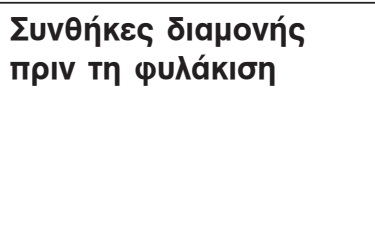 } & Móvos & 47 & 60,3 & 31 & 39,7 & \multirow{4}{*}{0,120} \\
\hline & Oıкоүع́veıа & 74 & 75,5 & 24 & 24,5 & \\
\hline & 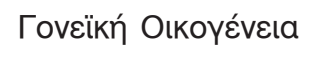 & 43 & 66,2 & 22 & 33,8 & \\
\hline & 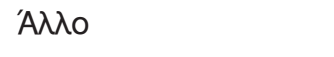 & 49 & 74,2 & 17 & 25,8 & \\
\hline \multirow{2}{*}{ 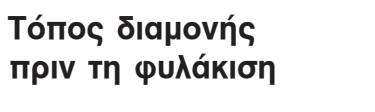 } & Aбтাкń & 146 & 70,5 & 61 & 29,5 & \multirow{2}{*}{0,454} \\
\hline & 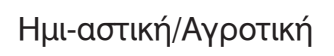 & 67 & 66,3 & 34 & 33,7 & \\
\hline \multirow{2}{*}{ 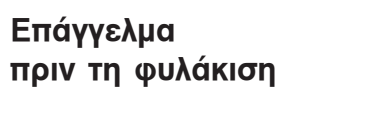 } & Nal & 190 & 67,9 & 90 & 32,1 & \multirow{2}{*}{0,119} \\
\hline & 'Oxı & 23 & 82,1 & 5 & 17,9 & \\
\hline \multirow[t]{2}{*}{ 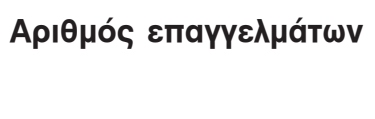 } & 'Eva & 128 & 67,7 & 61 & 32,3 & \multirow{2}{*}{0,946} \\
\hline & 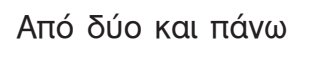 & 62 & 68,1 & 29 & 31,9 & \\
\hline \multirow{2}{*}{ 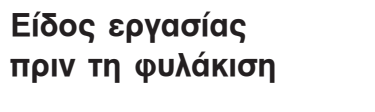 } & 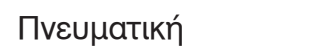 & 12 & 75,0 & 4 & 25,0 & \multirow{2}{*}{0,529} \\
\hline & Хеııроvaктıкń & 178 & 67,4 & 86 & 32,6 & \\
\hline \multirow{2}{*}{ 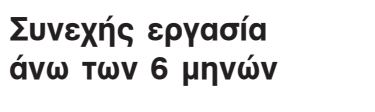 } & Nal & 149 & 67,1 & 73 & 32,9 & \multirow{2}{*}{0,604} \\
\hline & 'Oxl & 41 & 70,7 & 17 & 29,3 & \\
\hline \multirow[t]{2}{*}{ 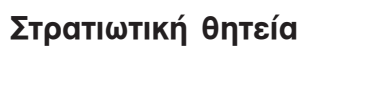 } & 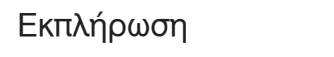 & 126 & 62,7 & 75 & 37,3 & \multirow{2}{*}{0,001} \\
\hline & 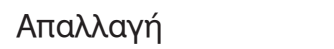 & 87 & 81,3 & 20 & 18,7 & \\
\hline
\end{tabular}

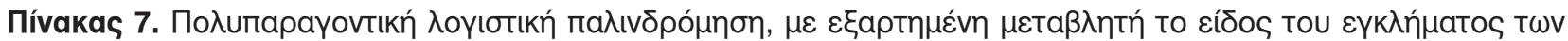

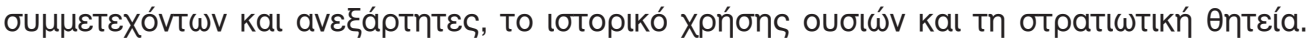

\begin{tabular}{|c|c|c|c|}
\hline & & OR $(95 \% \Delta E)^{*}$ & $\mathbf{p}$ \\
\hline \multirow[t]{2}{*}{ 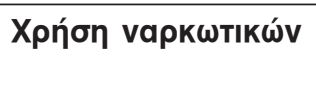 } & 'Oxı & 1,00 & \\
\hline & Naı & $0,35(0,20-0,64)$ & 0,001 \\
\hline \multirow[t]{2}{*}{ 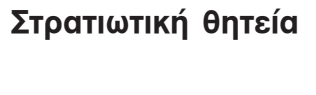 } & 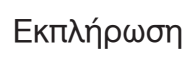 & 1,00 & \\
\hline & 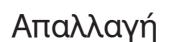 & $0,51(0,28-0,93)$ & 0,029 \\
\hline
\end{tabular}

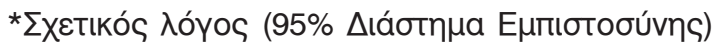

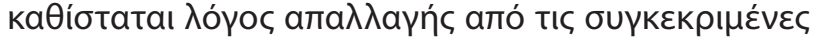

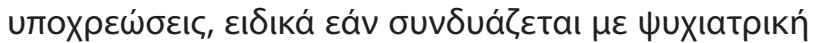

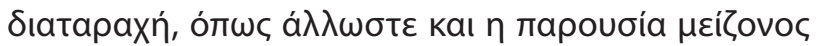

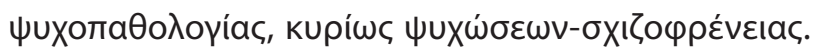

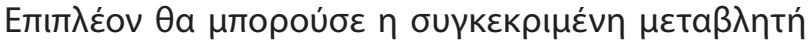

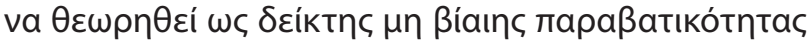

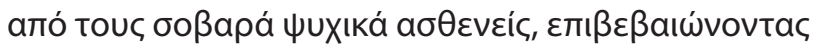

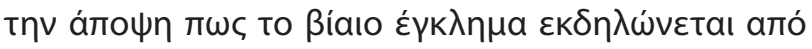

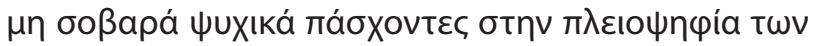

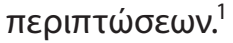

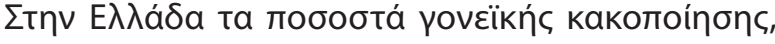

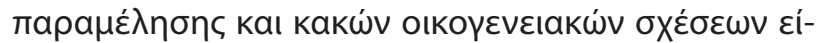

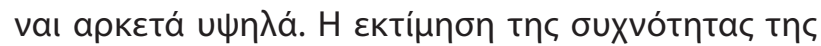

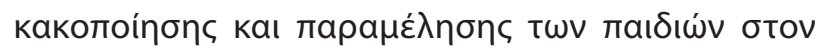

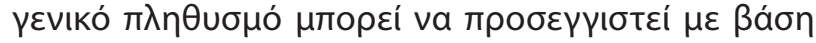

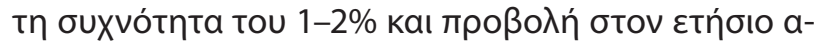

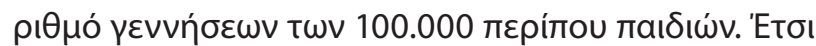

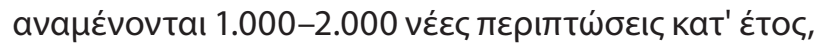

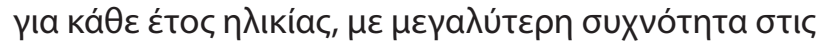

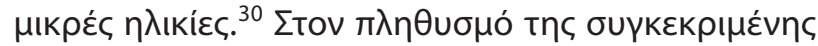




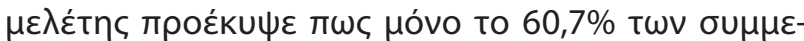

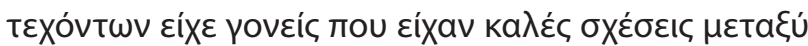

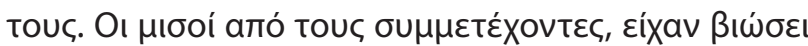

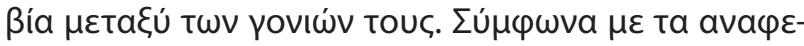

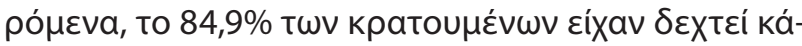

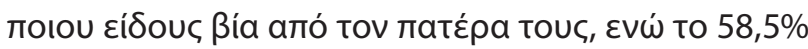

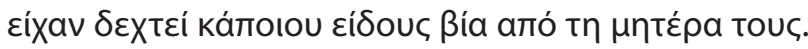

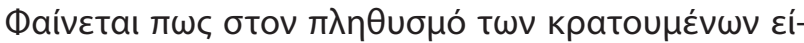

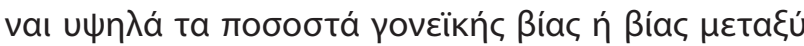

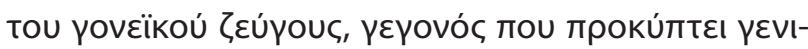

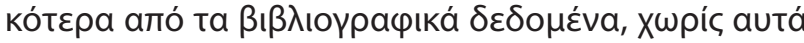

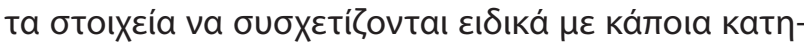

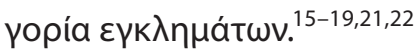

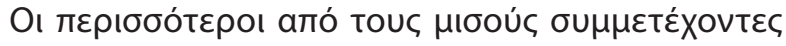

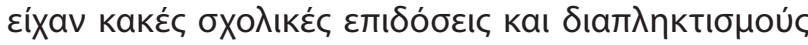

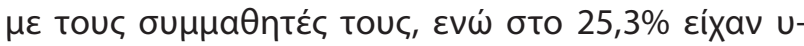

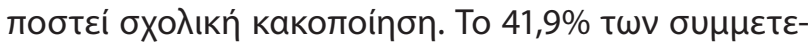

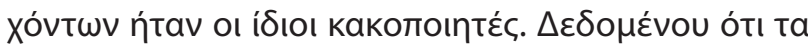

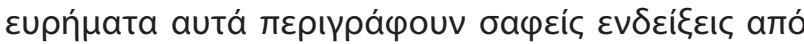

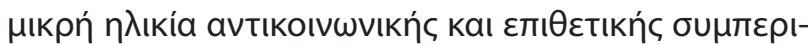

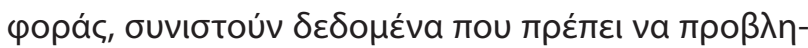

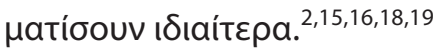

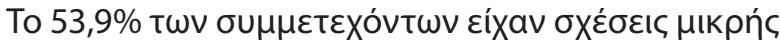

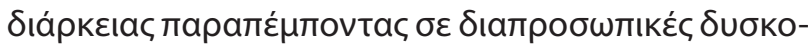

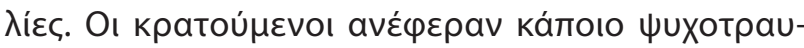

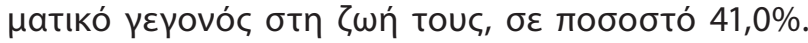

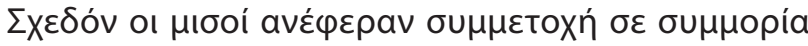

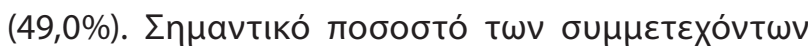

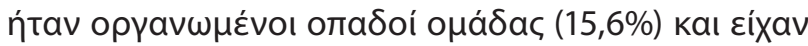

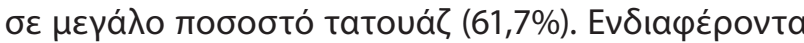

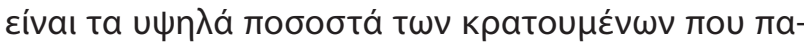

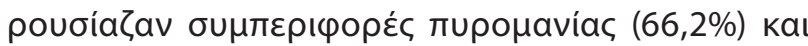

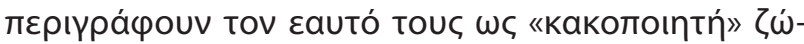

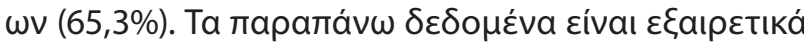

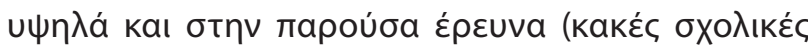

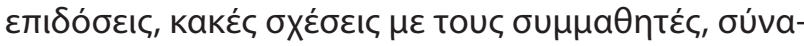

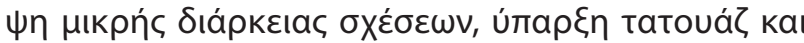

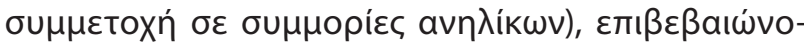

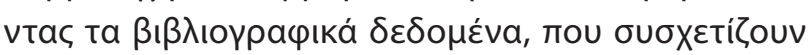

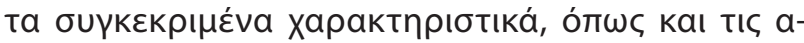

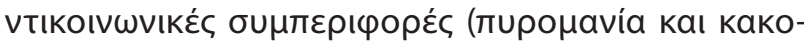

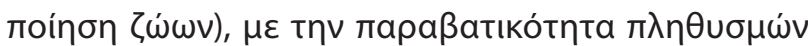

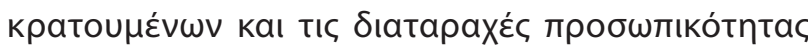

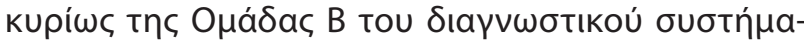
Toc DSM. ${ }^{1,2,13,14,20,31-33}$

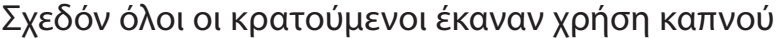
кaı a

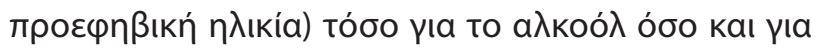

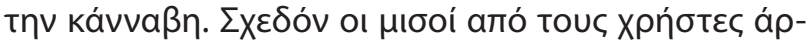

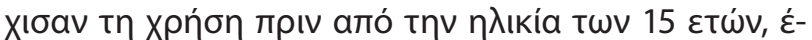

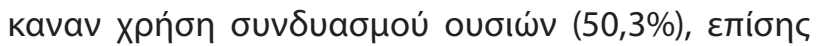

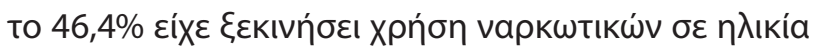

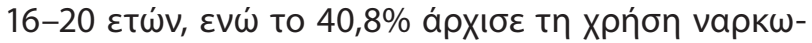

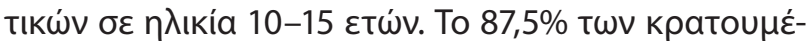

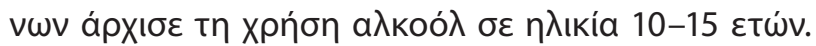

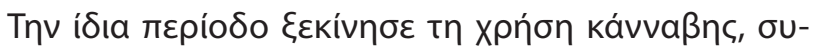

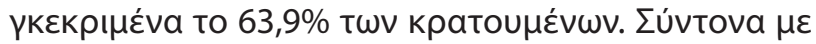

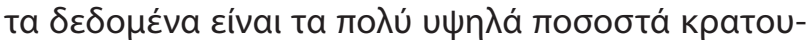

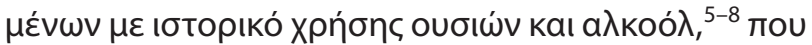

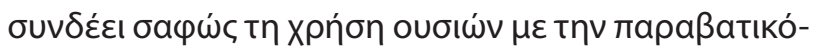

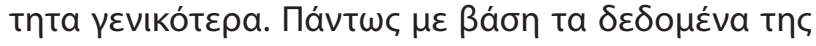

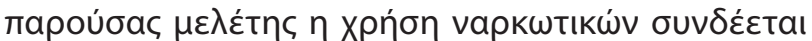

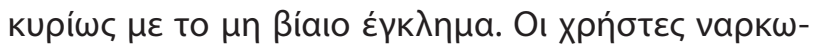

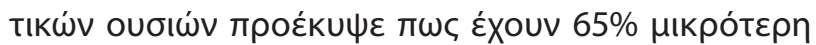

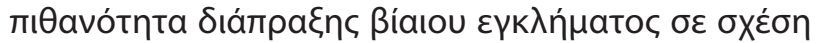

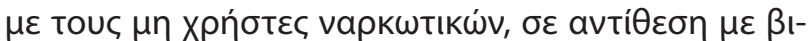

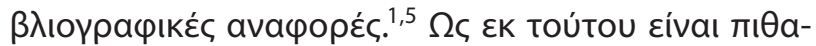

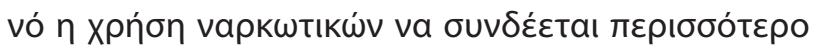

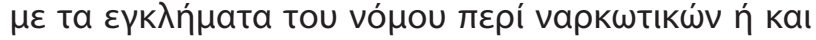

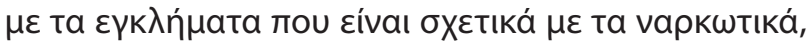

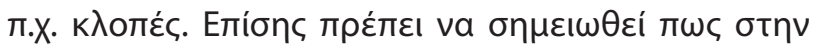

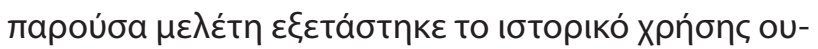

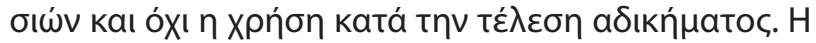

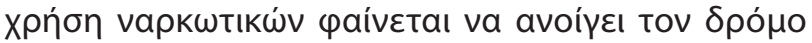

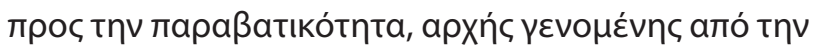

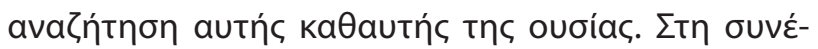

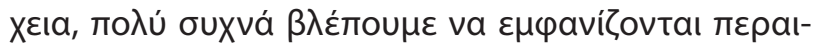

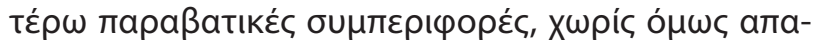

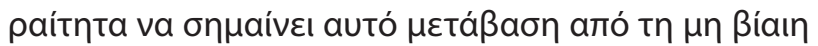

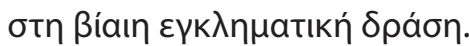

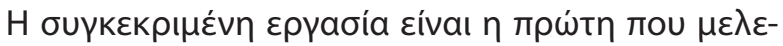

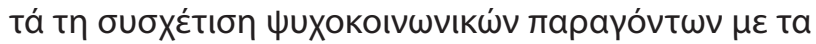

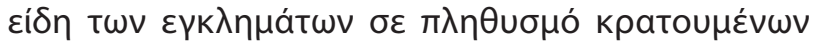

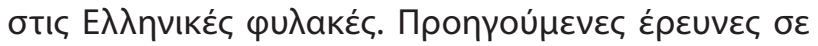

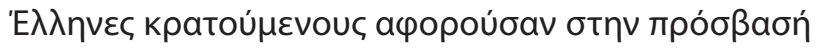

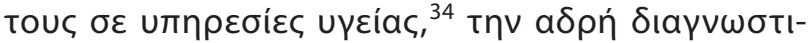

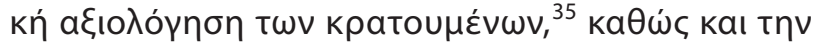

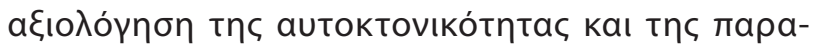

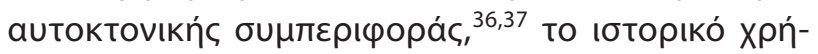

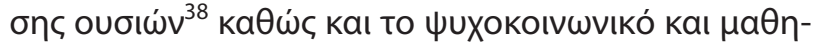

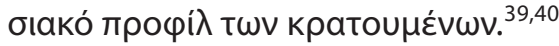




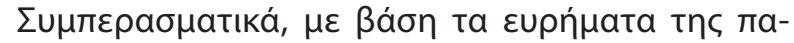

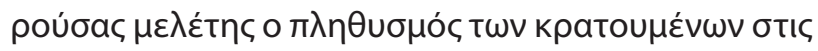

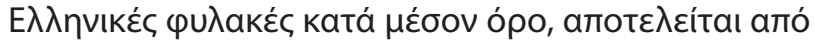

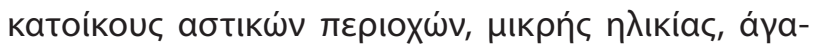

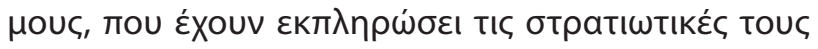

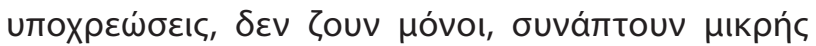

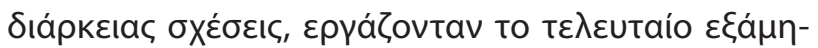

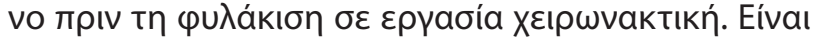

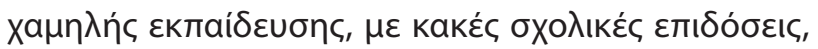

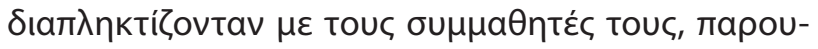

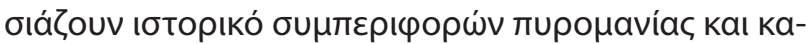

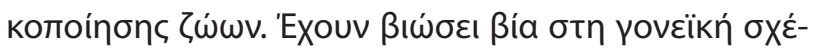

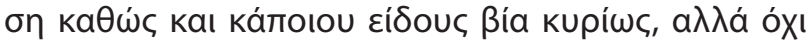

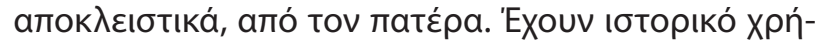

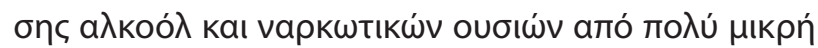

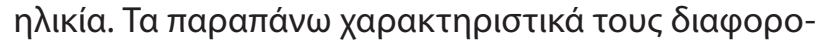

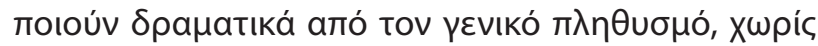

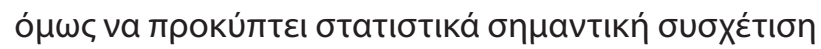

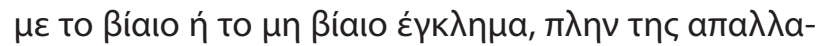

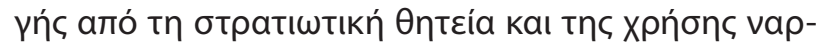

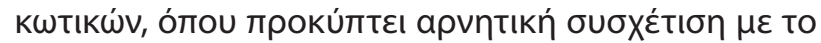

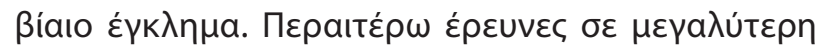

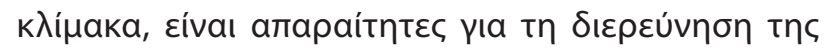

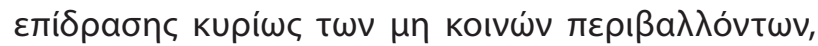

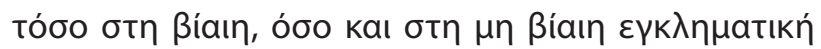

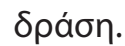

\title{
Prisoners in Greek prisons: Correlation of demographic and psychosocial data with committed crimes
}

\author{
A. Apostolopoulos, ${ }^{1}$ I. Michopoulos, ${ }^{1}$ E. Rizos, ${ }^{1}$ V. Manthou, ${ }^{3}$ \\ G. Tzeferakos, ${ }^{1}$ G. Kalemi, ${ }^{1}$ Ch. Papageorgiou, ${ }^{2}$ A. Douzenis ${ }^{1}$ \\ '2nd Psychiatric Department of the National and Kapodistrian University of Athens, "Attikon" Hospital, Athens, \\ ${ }^{2} 1$ st Psychiatric Department of the National and Kapodistrian University of Athens, Aeginition Hospital, Athens, \\ ${ }^{3}$ Organization Against Drugs (OKANA), Athens, Greece
}

Psychiatriki 2018, 29:137-148

Previous literature shows an association between several psychosocial factors or life events in general and delinquency. Factors such as gender, cannabis and drugs use are firmly connected to delinquency. Similarly, interpersonal violent behavior appears to be more frequent in people with lower socioeconomic status and people with lower education. The association of these factors with the violent or non-violent crimes, especially in Greek research literature, is very limited. The present study is an attempt to examine in a Greek prison population the correlation of demographic and psychosocial factors with violent and non-violent crime. The prison population sample comprised of 308 males from a total of 1300 prisoners, aged between 18 and 77 years old. The survey was conducted from January 2012 until August 2013 in Korydallos and Domokos prisons. In our prison population sample most of the crimes were non-violent. The prisoners were urban dwellers, of young age, were not married and were in short-term relationships on average. They had completed their military obligations, were not live alone, and have been working in the last six months before being imprisoned, in manual labor. They had low-grade education and poor school achievements, had been brawling with classmates and had history of antisocial behavior (liked to "put fire" and abuse animals). They report good relationships with their parents; however, they had experienced violence in parental relationships and some 
kind of violence, mainly by the father and secondarily by the mother. They have not been involved in gangs necessarily and have a history from a young age, of alcohol, cannabis and drugs use. Cannabis use history was reported by 208 prisoners (67.5\%) and 133 (63.9\%) of them started using at the age of 10-15 years old. A total of 179 prisoners (58.5\%) reported a history of drug use, about half of them (50.3\%) reported being addicted to a combination of drugs. For $40.8 \%(n=73)$ drug use was initiated in the age of $10-15$ years old, while the largest percentage (46.4\%) of prisoners mentioned as starting age 16-20 years old. Although the above features underline the great differences between prison population and the general population, there are no significant associations of these factors with violent or non-violent crime. However, the prisoners with drug use history were $65 \%$ less likely to have been sentenced for violent crime. Also, the prisoners exempted from their military duties, were $49 \%$ less likely to have committed violent crime.

Key words: Prisoners, demographic factors, psychosocial factors, crime.

\section{BıBntoypacpía}

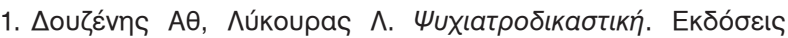
Пaoxaגíon, AӨńva, 2008

2. Freudenberg N, Moseley J, Labriola M, Daniels J, Murrill C. Comparison of health and social characteristics of People leaving New York City jails by age, gender, and race/ethnicity: implications for public health interventions. Publ Health Rep 2007, 122:733-743, doi:10.1177/003335490712200605

3. Içli TG, Seydioğullari I, Tatlidil H, Coban S, Sever H, Süeroğlu U. Profiling property criminals in Turkey. Int $\mathrm{J}$ Offender Ther Comp Criminol 2010, 54:640-655, doi:10.1177/0306624X09337390

4. Greenberg GA, Rosenheck RA. Jail incarceration, homelessness, and mental health: a national study. Psychiatr Serv 2008, 59(2):170-177, doi: 10.1176/ps.2008.59.2.170

5. Friedman AS, Glassman K, Terras BA. Violent behavior as related to use of marijuana and other drugs. $J$ Addict Dis 2001 , 20:49-72, doi:10.1300/J069v20n01 06

6. el-Guebaly N, Lee M. Alcholism and gun control. Can Psychiatr Assoc J 1977, 22:243-251, PMID: 890644

7. Wootton L, Buchanan A, Leese M, Tyrer P, Burns T, Creed $\mathrm{F}$ et al. Violence in psychosis: estimating the predictive validity of readily accessible clinical information in a community sample. Schizophr Res 2008, 101:176-184, doi: 10.1016/j. schres.2007.12.490

8. Seena Fazel, John Danesh. Psychiatric comorbidity in forensic psychiatry. Serious mental disorder in 23000 prisoners: a systematic review of 62 surveys. Lancet 2002, 359:545-550 doi: 10.1016/S0140-6736(02)07740-1

9. Pulay AJ, Dawson DA, Hasin DS, Goldstein RB, Ruan WJ, Pickering RP et al. Violent behavior and DSM-IV psychiatric disorders: results from the national epidemiologic survey on alcohol and related conditions. J Clin Psychiatry 2008, 69:12-22, PMID: 18312033

10. Amdzaranda PA, Fatoye FO, Oyebanji AO, Ogunro AS, Fatoye GK. Factors associated with psychoactive substance use among a sample of prison inmates in Ilesa, Nigeria. Niger Postgrad Med J 2009, 16:109-114, PMID: 19606190

11. Haggård-Grann U, Hallqvist J, Långström N, Möller J. The role of alcohol and drugs in triggering criminal violence: a case-cross- over study. Addiction 2006, 101:100-108, doi: 10.1111/j.13600443.2005.01293.x

12. Hekansson A, Berglund M. Risk factors for criminal recidivism - a prospective follow-up study in prisoners with substance abuse. BMC Psychiatry 2012, 12:111, doi: 10.1186/1471-244X-12-111

13. Knittel AK, Snow RC, Griffith DM, Morenoff J. Incarceration and sexual risk: examining the relationship between men's involvement in the criminal justice system and risky sexual behavior. AIDS Behav 2013, 17:2703-2714, doi: 10.1007/s10461-0130421-4

14. Carr BG, Wiebe DJ, Richmond TS, Cheney R, Branas CC. A randomised controlled feasibility trial of alcohol consumption and the ability to appropriately use a firearm. Inj Prev 2009, 6:409-412, doi: 10.1136/ip.2008.020768

15. Lemos I, Favsca L. Psychosocial adversity, delinquent pathway and internalizing psychopathology in juvenile male offenders. Int J Law Psychiatry 2015, 42-43:49-57, doi: 10.1016/j. ijlp.2015.08.007

16. Neller DJ, Denney RL, Pietz CA, Thomlinson RP. The relationship between trauma and violence in a jail inmate sample. $J$ Interpers Violenc 2006, 21:1234-1241, doi: 10.1177/0886260506290663

17. Marcus RF, Gray L Jr. Close relationships of violent and nonvioIent African American delinquents. Violence Vict 1998, 13:31-46, PMID: 9650244

18. Miller KS, Knutson JF. Reports of severe psysical punishment and exposure to animal cruelty by inmates convicted of felonies and by university students. Child Aduse Negl 1997, 21:59-82, PMID: 9023023

19. Murray J, Farrington DP, Sekol I. Children's antisocial behavior, mental health, drug use, and educational performance after parental incarceration: a systematic review and meta-analysis. Psychol Bull 2012, 138:175-210, doi: 10.1037/a0026407

20. Marcus RF. Cross-sectional study of violence in emerging adulthood. Aggress Behav 2009, 35:188-202, doi: 10.1002/ab.20293

21. Spinhoven P, Elzinga BM, Van Hemert AM, de Rooij M, Penninx BW. Childhood maltreatment, maladaptive personality types and level and course of psychological distress: A six-year longi- 
tudinal study. J Affect Disord 2016, 191:100-108, doi: 10.1016/j. jad.2015.11.036

22. Beaver KM, Al-Ghamdi MS, Kobeisy AN, Alqurashi FH, Connolly EJ, Schwartz JA. The Association Between Psychopathic Personality Traits and Victimization and Exposure to Violence in a Sample of Saudi Arabian Youth. Psychiatr Q 2016, 87:217228, doi: 10.1007/s11126-015-9383-1

23. Van Zalk MH, Van Zalk N. Violent peer influence: The roles of self-esteem and psychopathic traits. Dev Psychopathol 2015, 27:1077-88, doi: 10.1017/S0954579415000693

24. Claes L, Tavernier G, Roose A, Bijttebier P, Smith SF, Lilienfeld SO. Identifying personality subtypes based on the five-factor model dimensions in male prisoners: implications for psychopathy and criminal offending. Int $J$ Offender Ther Comp Criminol 2014, 58:41-58, doi: 10.1177/0306624X12462013

25. Carlson M, Oshri A, Kwon J. Child maltreatment and risk behaviors: The roles of callous/unemotional traits and conscientiousness. Child Abuse Negl 2015, 50:234-243, doi: 10.1016/j. chiabu.2015.07.003.

26. Sergentanis TN, Sakelliadis El, Vlachodimitropoulos D, Goutas N, Sergentanis IN, Spiliopoulou CA et al. Does history of childhood maltreatment make a difference in prison? A hierarchical approach on early family events and personality traits. Psychiatry Res 2014, 220:1064-1070, doi: 10.1016/j.psychres.2014.10.019

27. Vaughn MG, Salas-Wright CP, DeLisi M, Qian Z. The antisocial family tree: family histories of behavior problems in antisocial personality in the United States. Soc Psychiatry Psychiatr Epidemiol. 2015, 50:821-831, doi: 10.1007/s00127-014-0987-9.

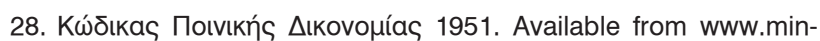
istryofjustice.gr

29. Kendler KS, Lönn SL, Sundquist J, Sundquist K. The role of marriage in criminal recidivism: a longitudinal and co-relative analysis. Epidemiol Psychiatr Sci 2017, 18:1-9, doi: 10.1017/ S2045796016000640

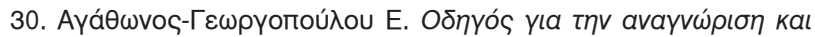

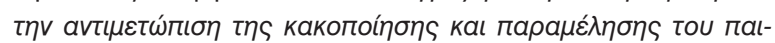

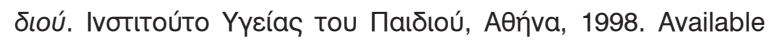
from www.ich-mhsw.gr

31. Roberts TA, Ryan SA. Tattooing and high-risk behavior in adolescents. Pediatrics 2002, 110:1058-1063, PMID:12456900

32. Hensley C, Tallichet SE. Animal cruelty motivations: assessing demographic and situational influences. $J$ Interpers Violence 2005, 20):1429-1443, doi: 10.1177/0886260505278714

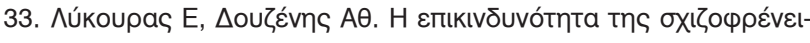
ac. Uuхıатрıќ́ 2011, 22:105

34. Geitona M, Milioni SO. Health status and access to health services of female prisoners in Greece a cross- sectional survey. BMC Health Serv Res 2016, 16:243, doi: 10.1186/s12913016-1506-3

35. Alevizopoulos G, Igoumenou A. Psychiatric disorders and criminal history in male prisoners in Greece. Int $J$ Law Psychiatry. 2016, 47:171-175, doi: 10.1016/j.jilp.2016.04.003

36. Sakelliadis El, Vlachodimitropoulos DG, Goutas ND, Panousi PI, Logiopoulou AP, Delicha EM et al. Forensic investigation of suicide cases in major Greek correctional facilities. J Forensic Leg Med. 2013, 20:953-958, doi: 10.1016/j.jlm.2013.08.009

37. Sakelliadis EI, Papadodima SA, Sergentanis TN, Giotakos O, Spiliopoulou CA. Self injurious behavior among Greek male prisoners: prevalence and risk factors. Eur Psychiatry 2010, 25:151-158, doi: 10.1016/j.eurpsy.2009.07.014

38. Fotiadou M, Livaditis M, Manou I, Kaniotou E, Samakouri M, Tzavaras N et al. Self-reported substance misuse in Greek male prisoners. EurAddictRes 2004, 10:56-60, doi: 10.1159/000076114

39. Maniadaki K, Kakouros E. Social and mental health profiles of young male offenders in detention in Greece. Crim Behav Ment Health 2008, 18:207-215, doi: 10.1002/cbm.698

40. Zakopoulou V, Pashou T, Tzavelas P, Christodoulides P, Anna M, lliana K. Learning difficulties: a retrospective study of their co morbidity and continuity as indicators of adult criminal behaviour in 18-70-year-old prisoners. Res Dev Disabil 2013, 34:3660-3671, doi: $10.1016 /$ j.ridd.2013.08.033

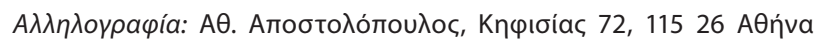
$\operatorname{T\eta \lambda .~213-03~} 62$ 916, 6944-721 170 e-mail: th.apostolopoulos@gmail.com 


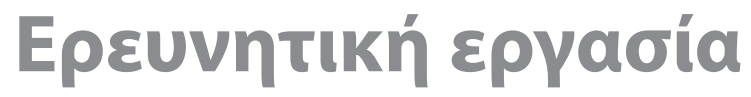 Research article
}

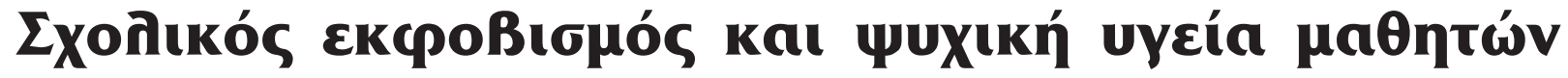

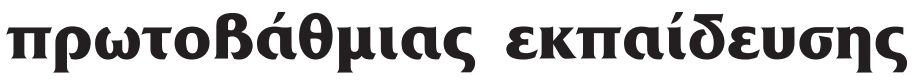

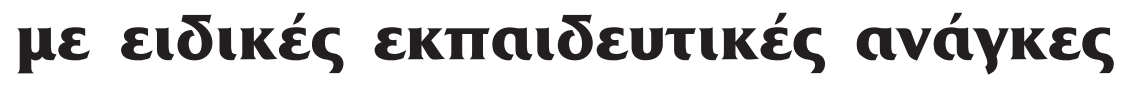

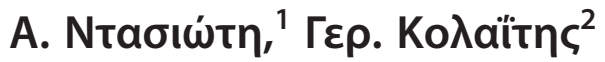

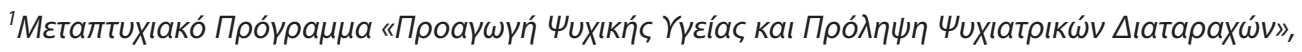

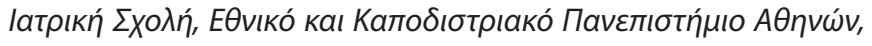

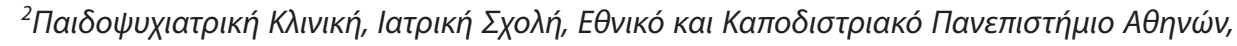

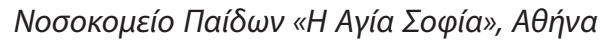

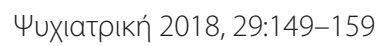

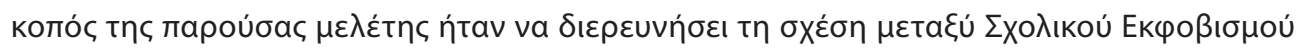

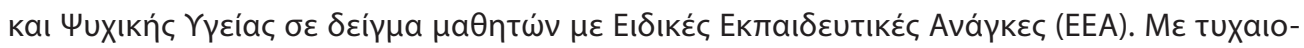

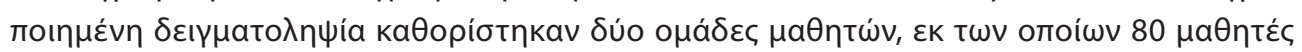

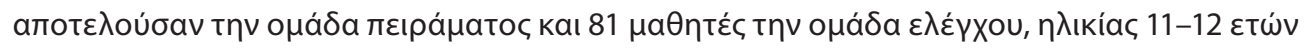

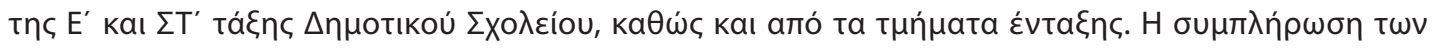

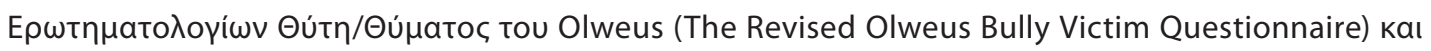

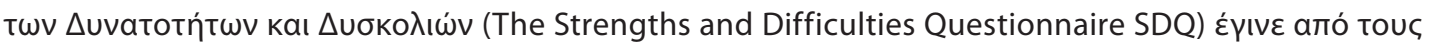

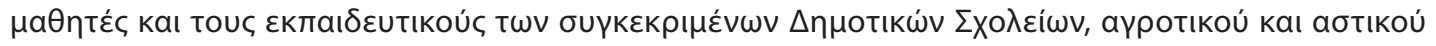

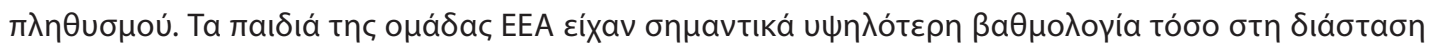

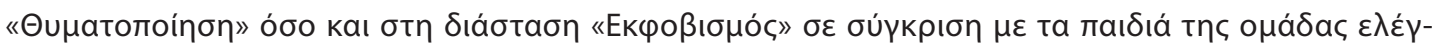

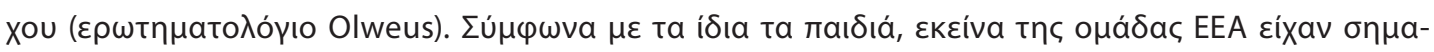

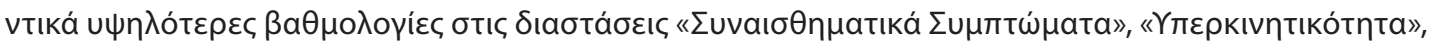

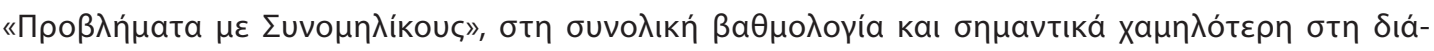

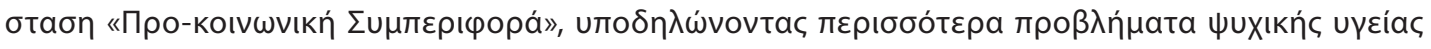

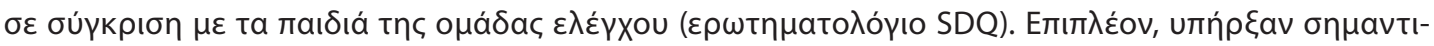

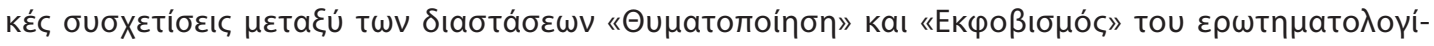

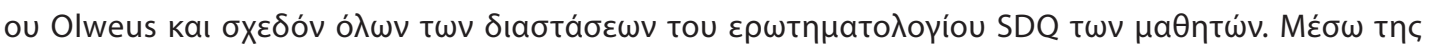




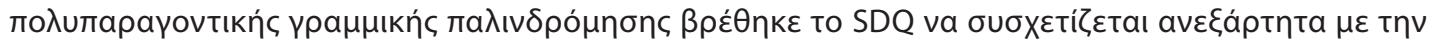

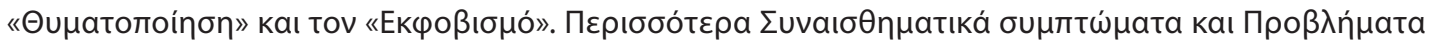

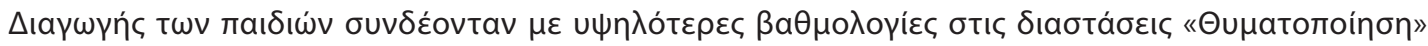

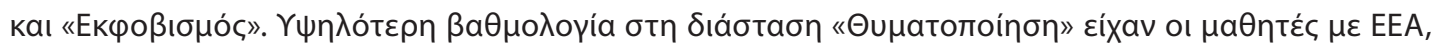

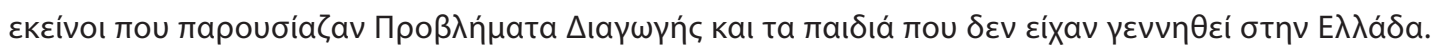

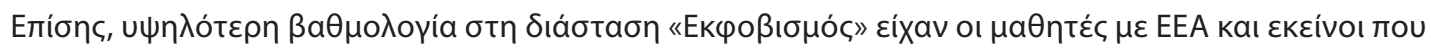

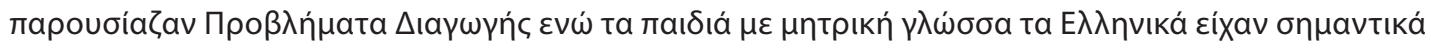

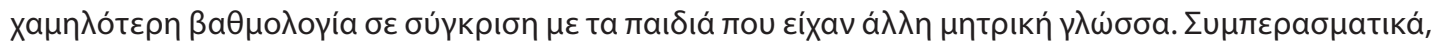

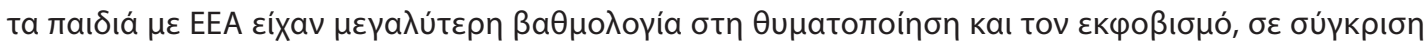

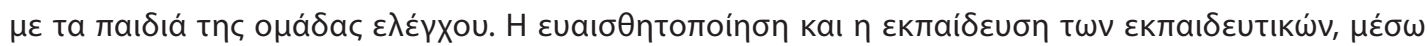

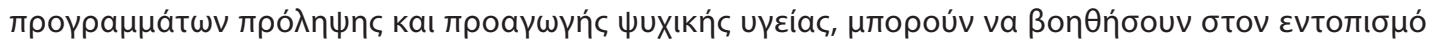

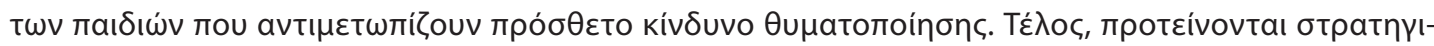

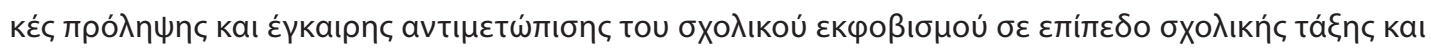

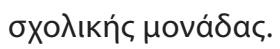

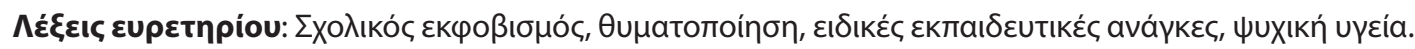

\section{Eigaywyń}

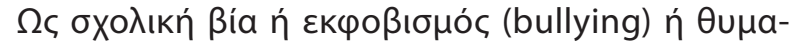

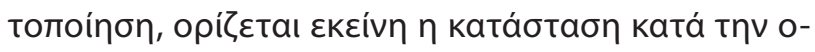

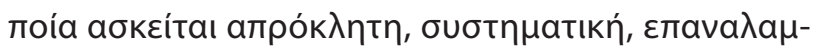

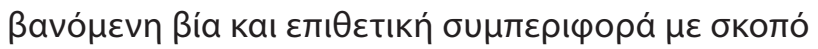

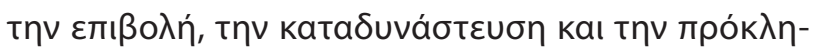

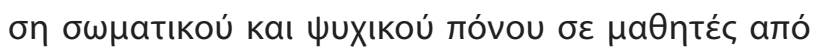

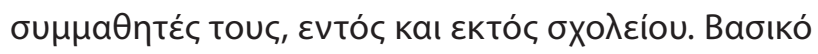

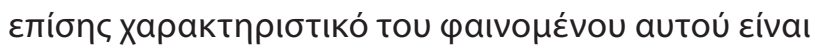

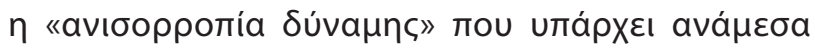

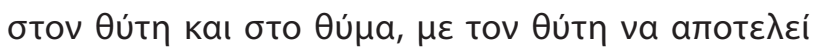

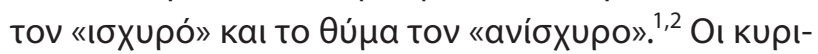

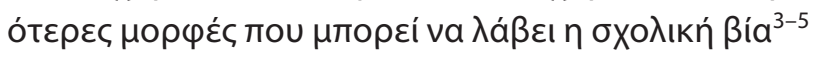

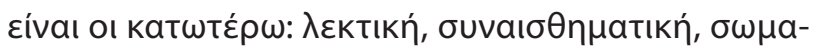

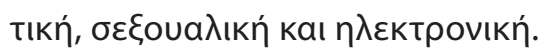

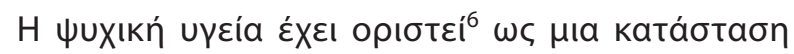

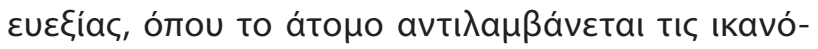

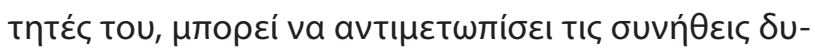

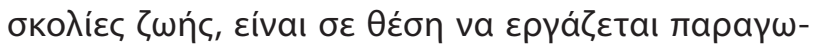

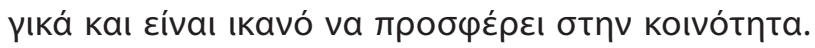

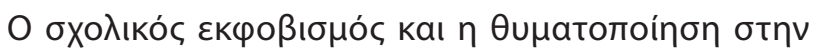

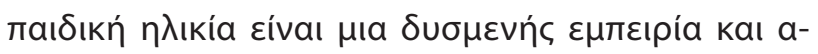

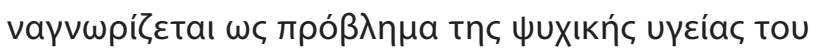

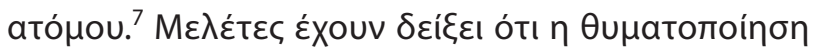

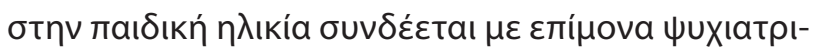

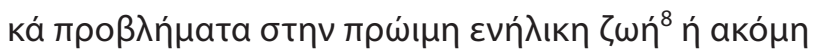

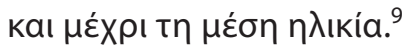

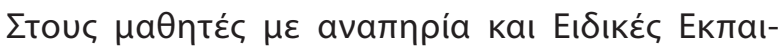

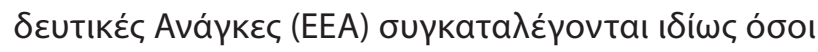

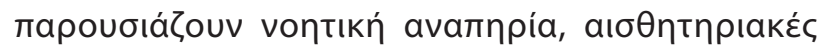

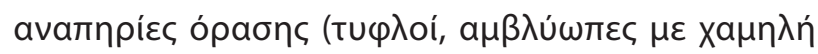

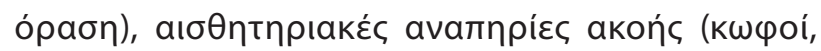

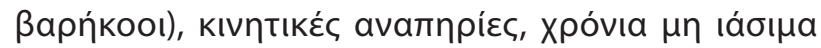

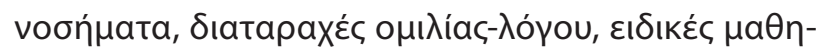

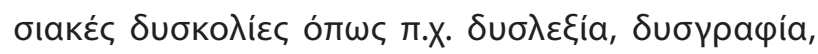

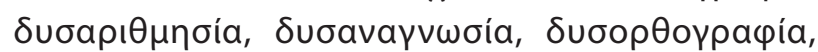

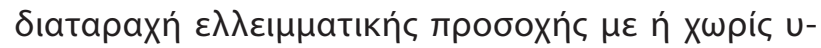

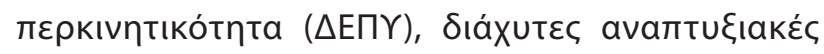

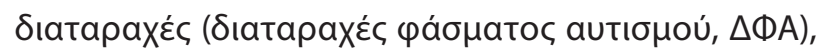

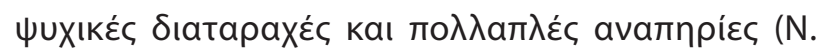
3699/08).

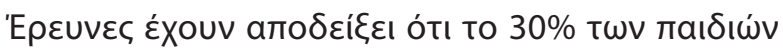

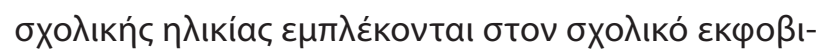

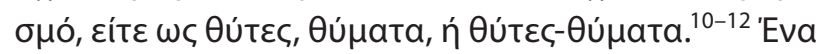

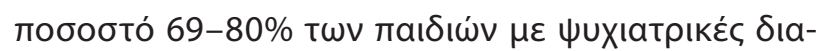

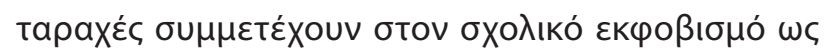

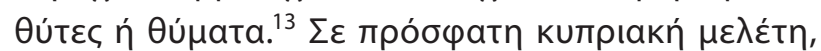

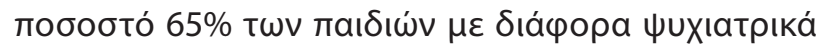

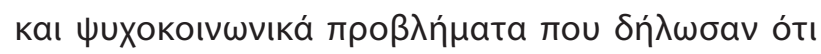

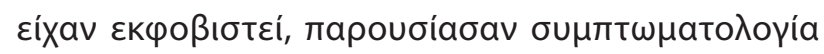

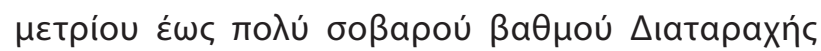

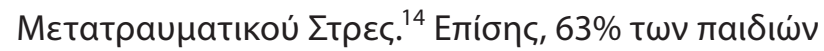

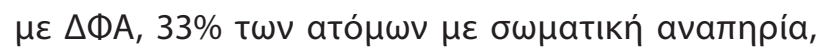

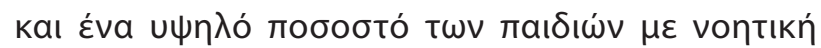

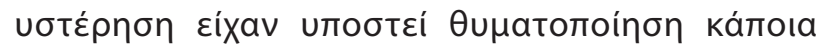




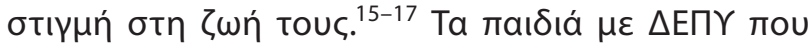

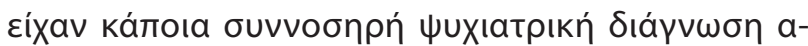

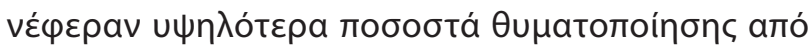

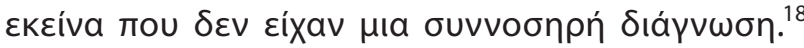

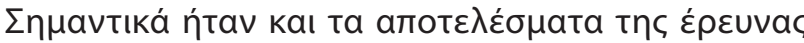

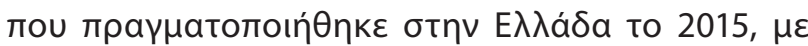

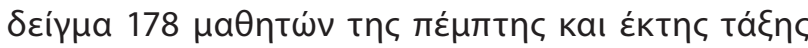

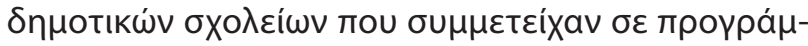

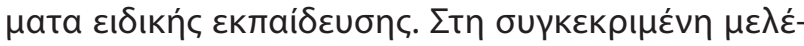

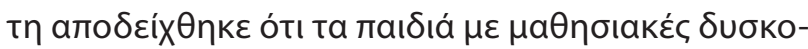

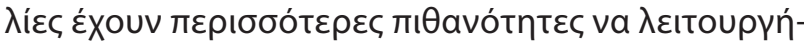

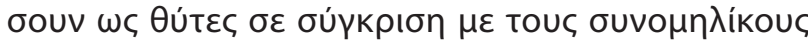

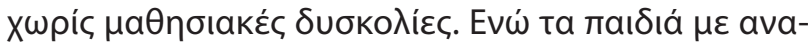

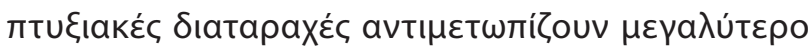

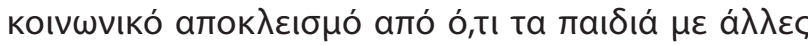

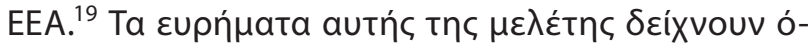

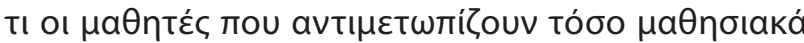

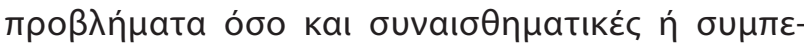

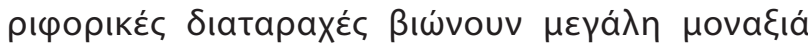

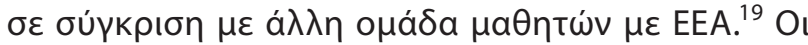

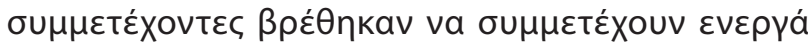

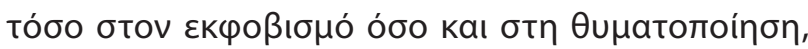

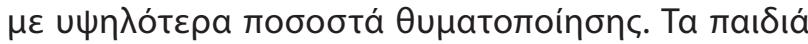

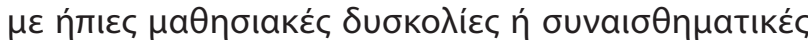

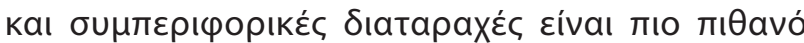

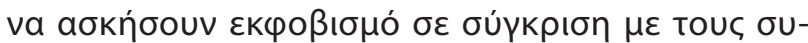

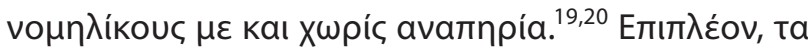

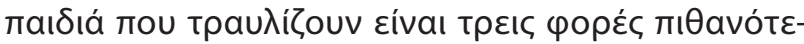

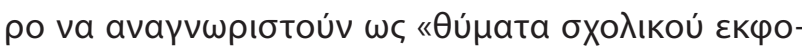
ßıॅนоú». ${ }^{21}$

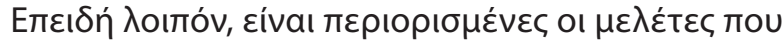

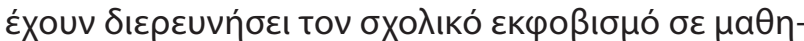

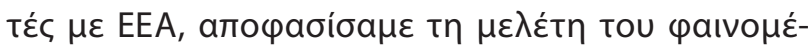

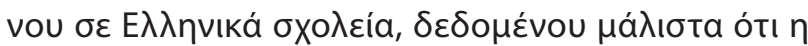

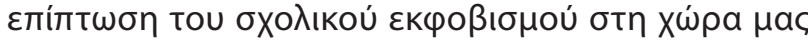

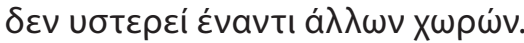

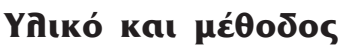

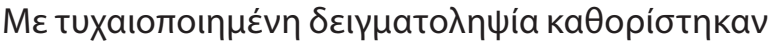

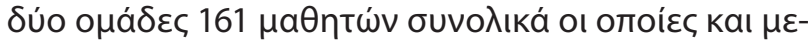

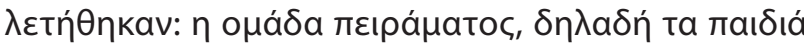

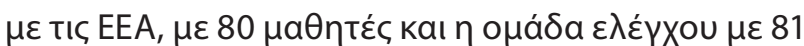

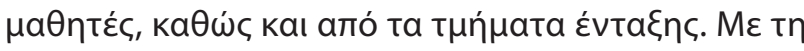

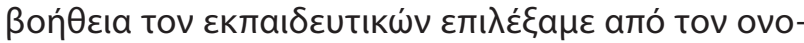

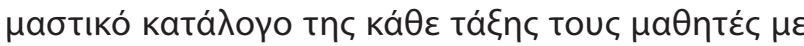

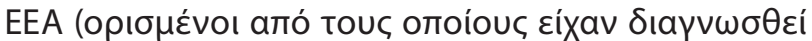

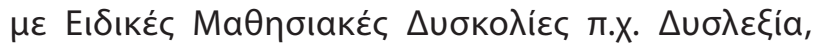

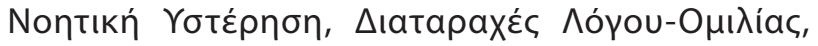

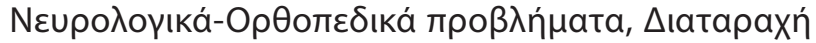

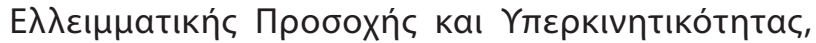

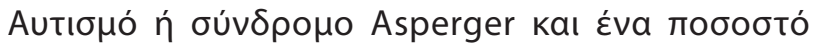

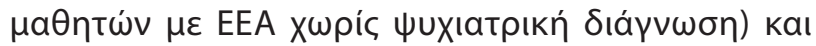

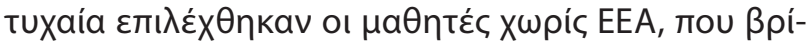

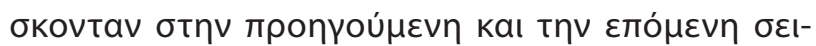

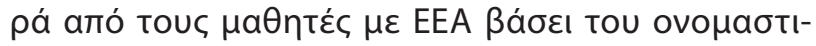

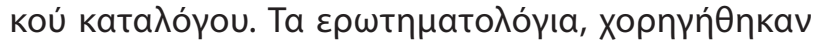

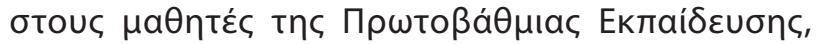

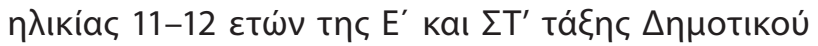

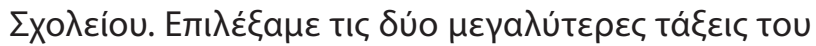

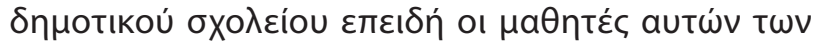

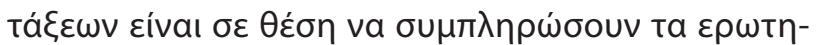

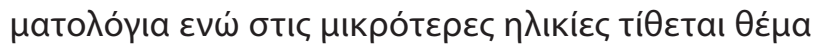

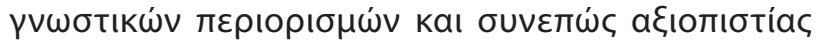

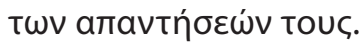

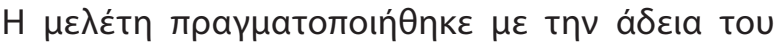

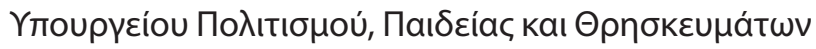

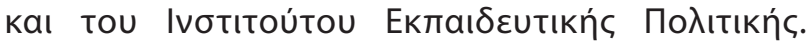

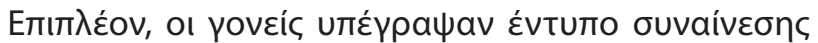

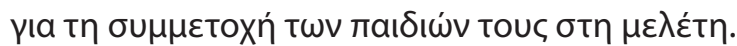

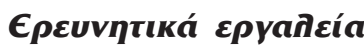

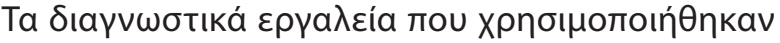
ท́tav:

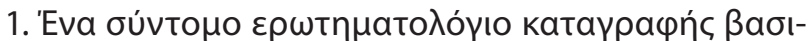

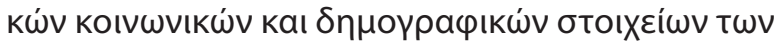

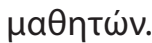

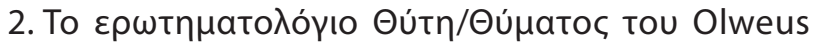
(The Revised Olweus Bully Victim Questionnaire)

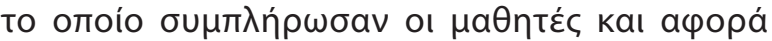

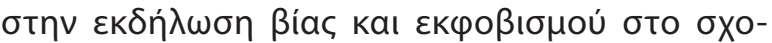

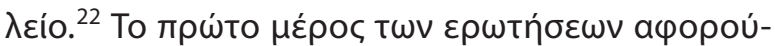

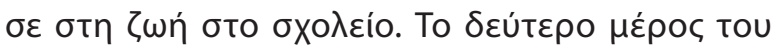

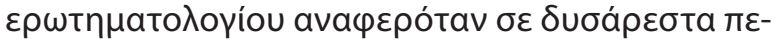

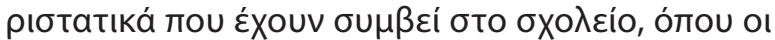

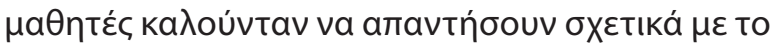

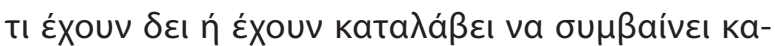

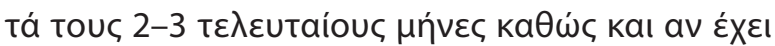

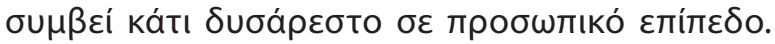

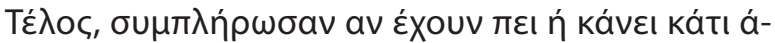

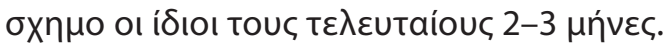




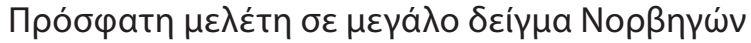

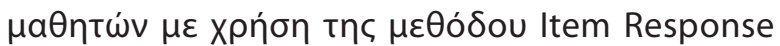

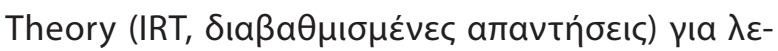

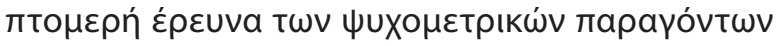

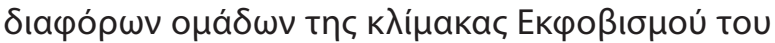

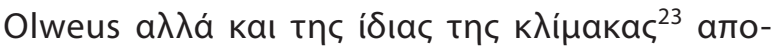

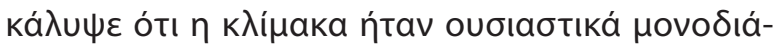

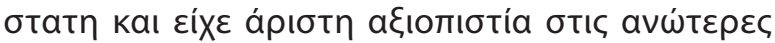

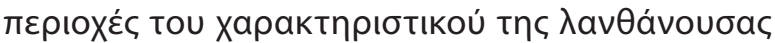

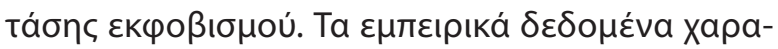

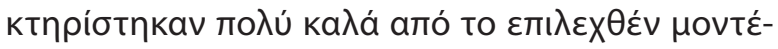

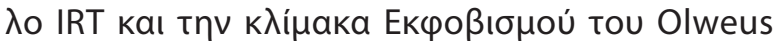

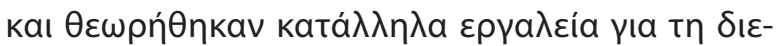

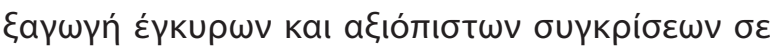

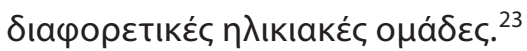

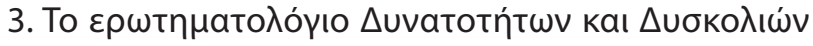
(The Strengths and Difficulties Questionnaire

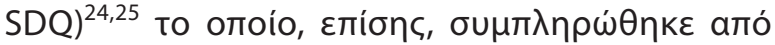

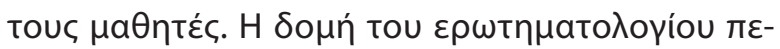

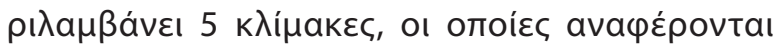

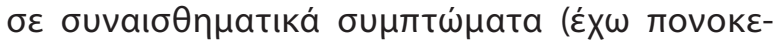

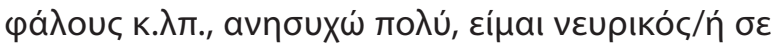

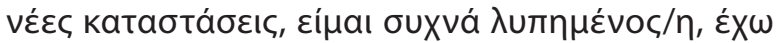

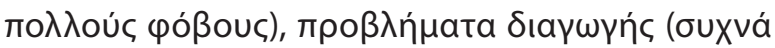

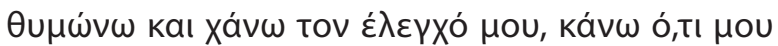

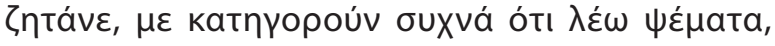

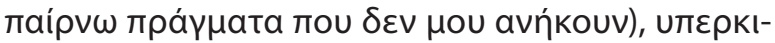

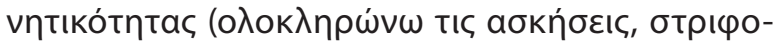

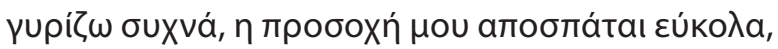

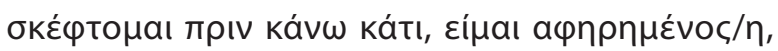

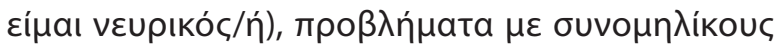

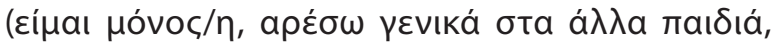

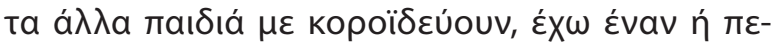

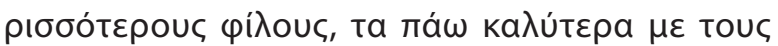

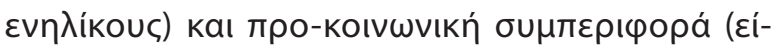

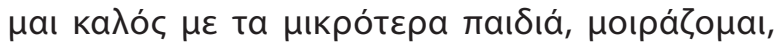

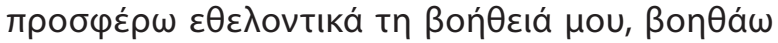

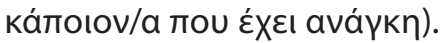

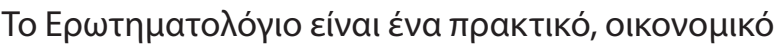

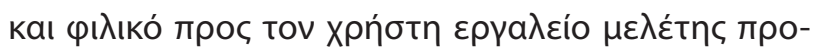

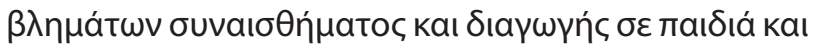

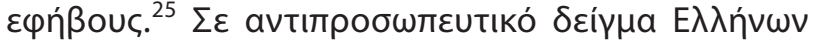

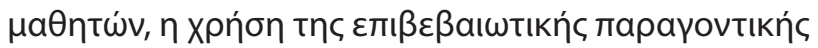

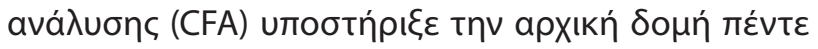

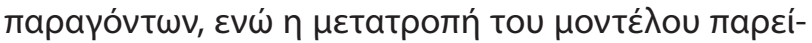

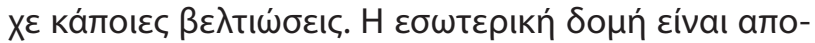

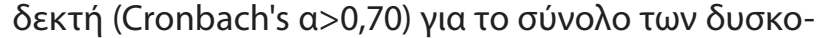

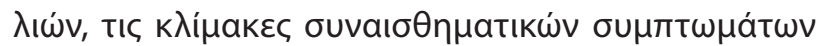

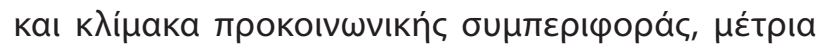

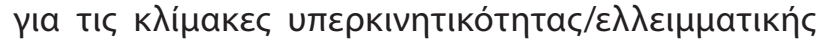

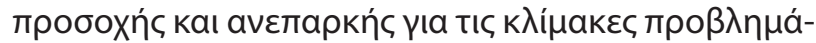

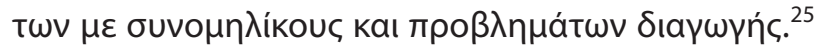

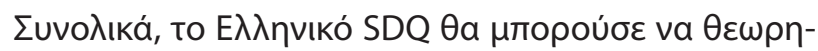

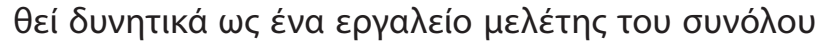

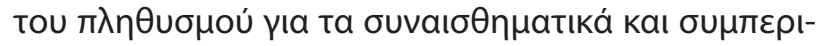

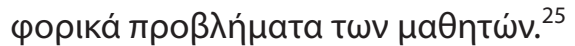

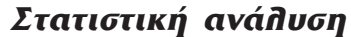

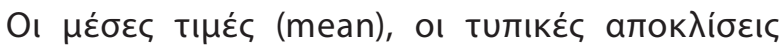

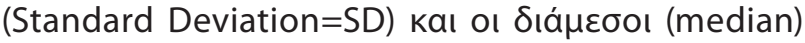

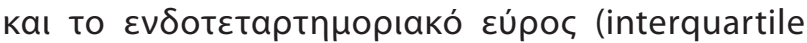

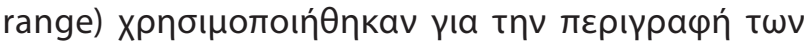

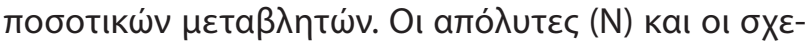

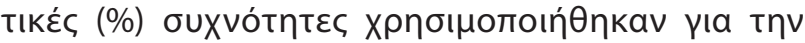

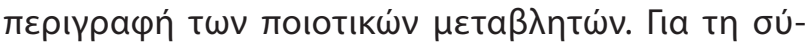

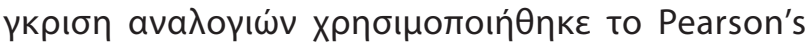
$\mathrm{x}^{2}$ test ń тo Fisher's exact test ómou ńtav amapaítn-

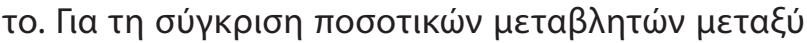

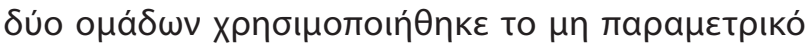

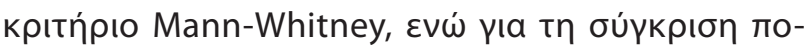

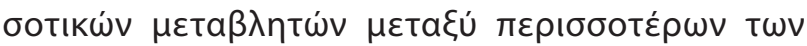

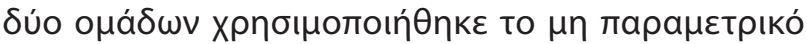

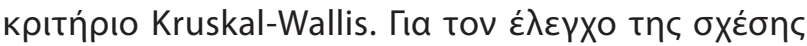

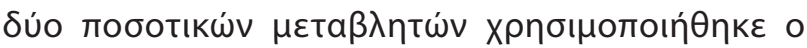

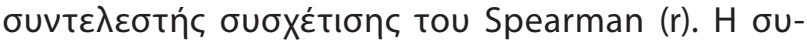

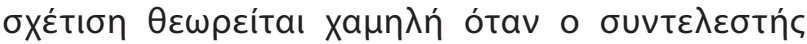

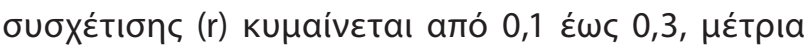

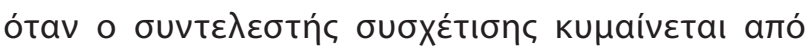

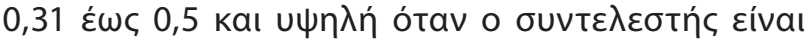

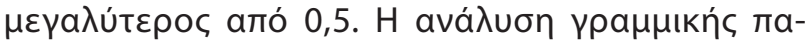

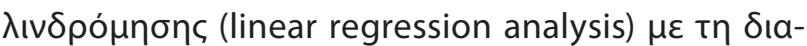

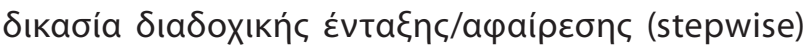

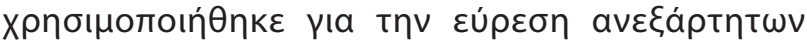

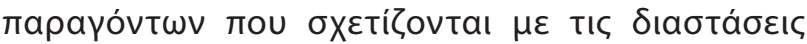

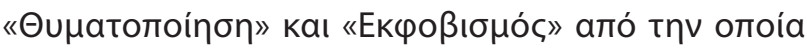

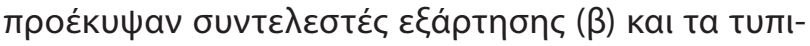
кá $\sigma \varphi a ́ \lambda \mu a t a ́$ touc (standard errors=SE). H avá $\lambda \cup \sigma \eta$

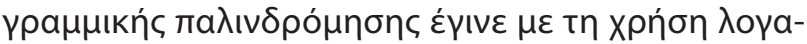

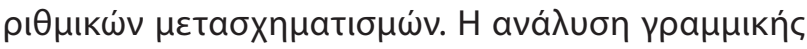

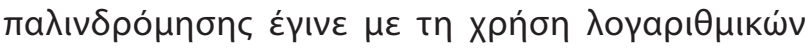

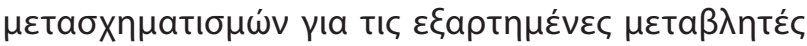

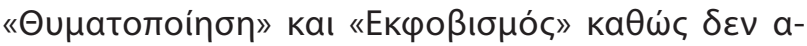




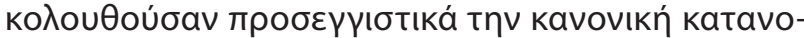

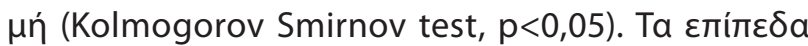

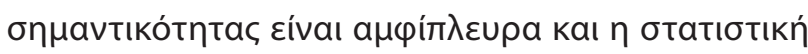

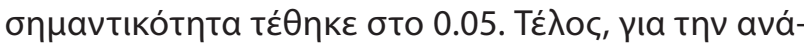

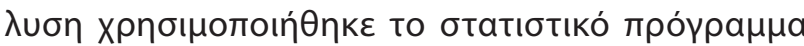
SPSS 19.0

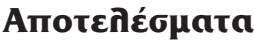

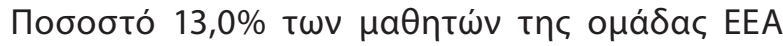

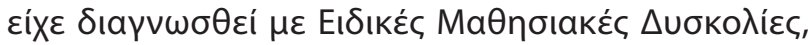

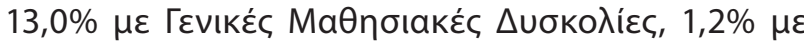

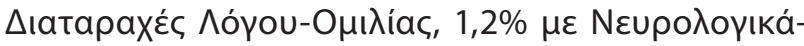

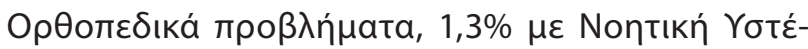

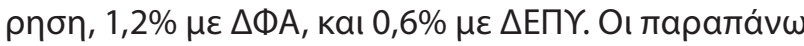

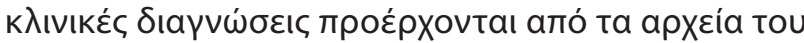

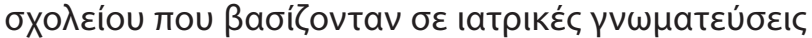

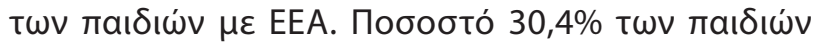

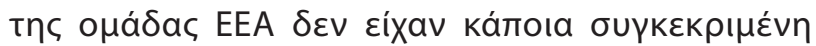

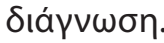

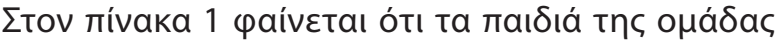

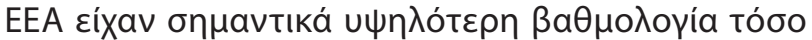

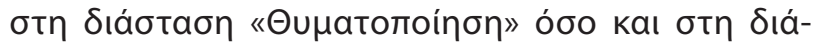

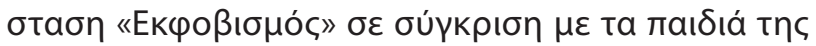
o $\mu a ́ \delta a c ~ \varepsilon \lambda \varepsilon ́ \gamma X O U$.

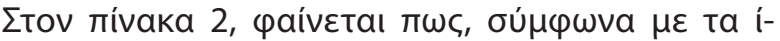

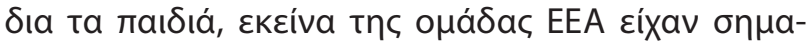

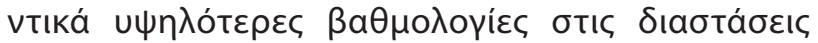

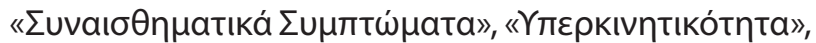

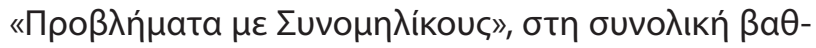

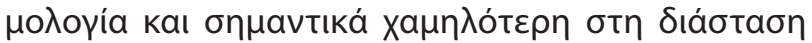

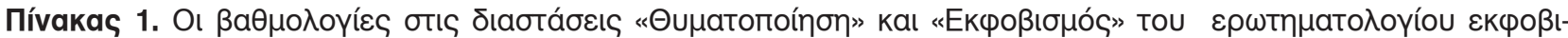
бuoú (Olweus).

\begin{tabular}{|c|c|c|c|c|c|}
\hline & \multicolumn{4}{|c|}{ O„áda } & \multirow{3}{*}{$\begin{array}{l}\text { p Mann-Whitney } \\
\text { test }\end{array}$} \\
\hline & \multicolumn{2}{|c|}{ Eגع́YXOU } & \multicolumn{2}{|c|}{ EEA } & \\
\hline & 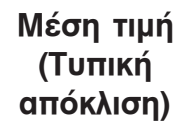 & 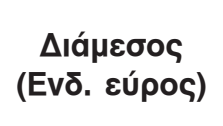 & 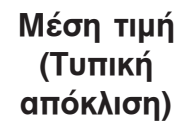 & 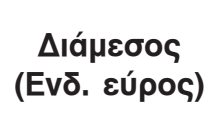 & \\
\hline 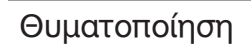 & $2,32(4.32)$ & $0(0-2)$ & $3,7(5,08)$ & $1(0-5,5)$ & 0,018 \\
\hline Екчоßıбно́ & $0,56(2.05)$ & $0(0-0)$ & $1,99(4,81)$ & $0(0-1)$ & 0,006 \\
\hline
\end{tabular}

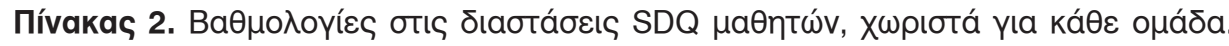

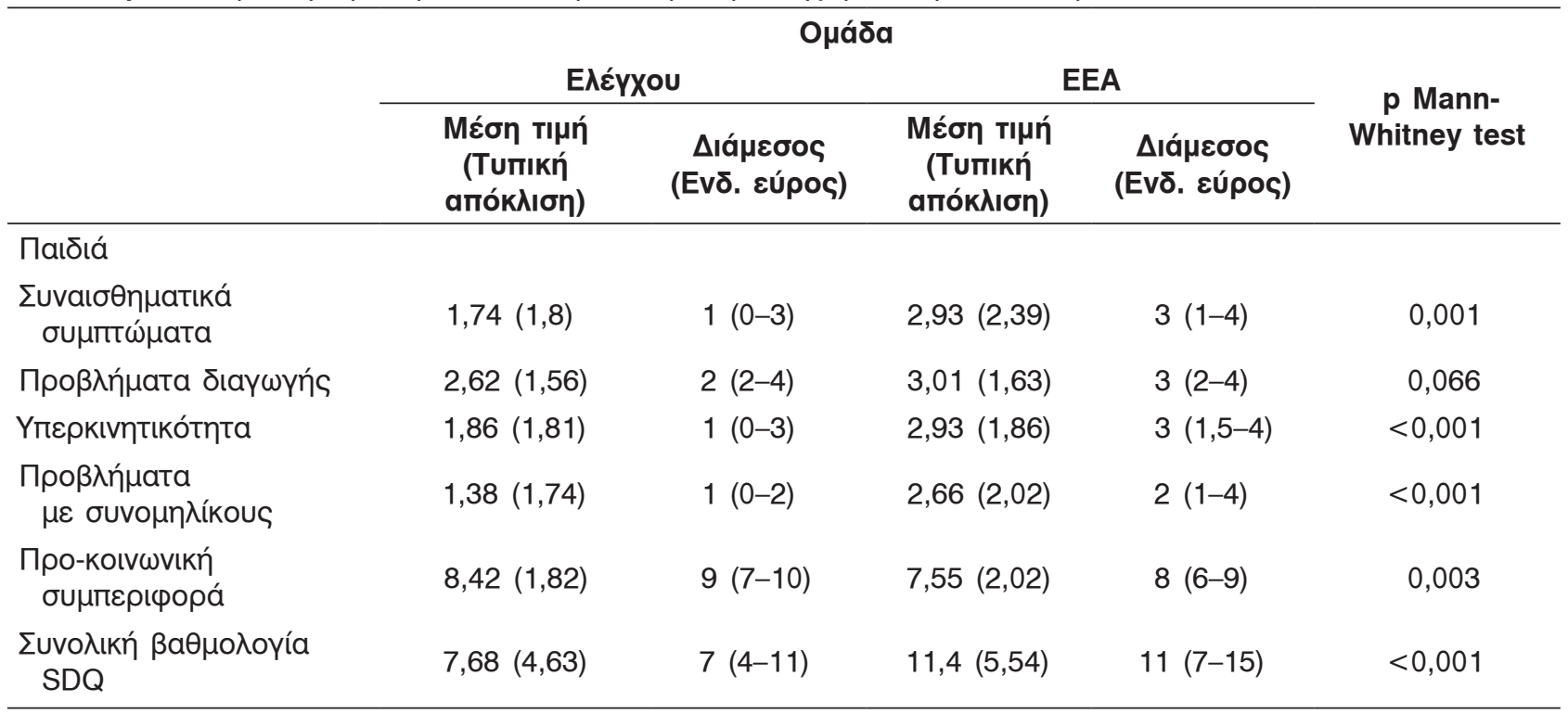




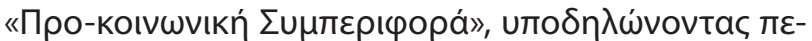

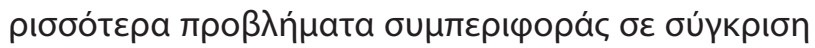

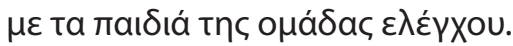

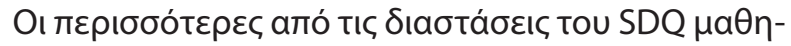

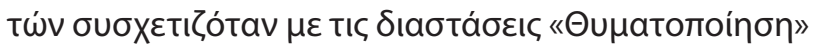

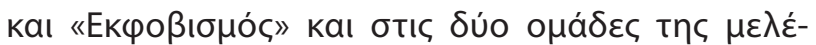

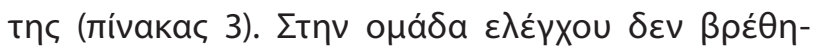

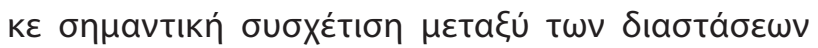

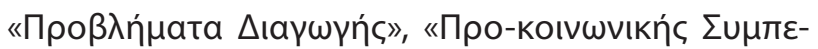

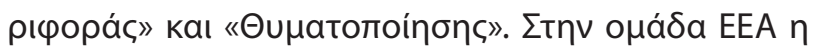

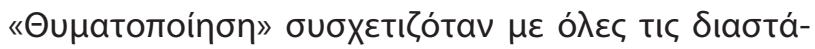

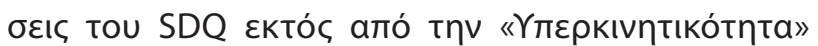

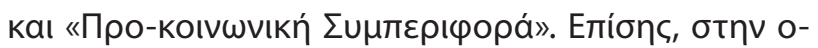

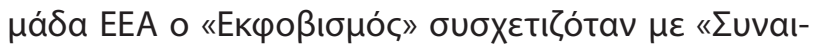

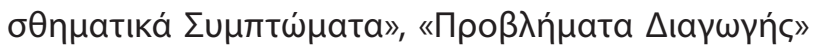

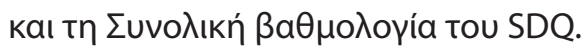

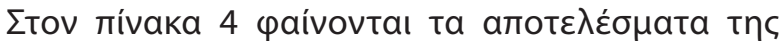

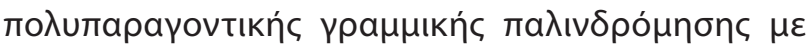

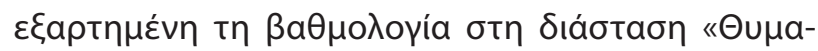

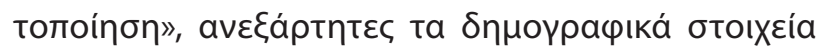

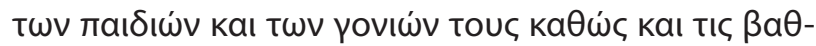

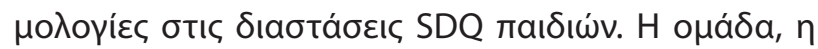

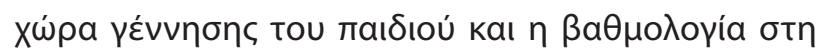

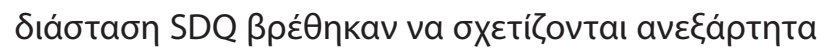

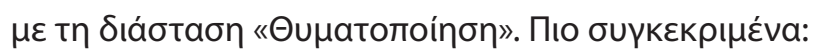

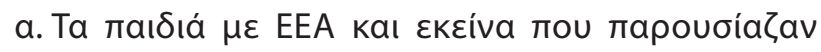

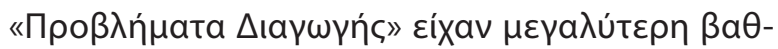

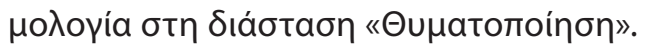

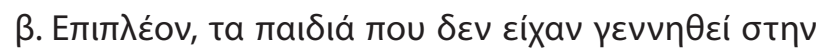

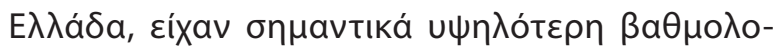

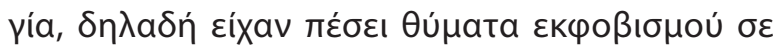

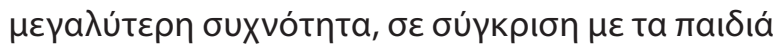

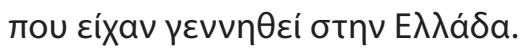

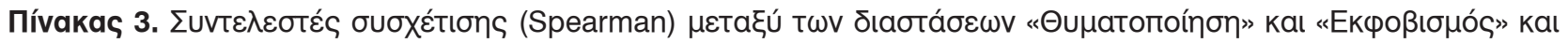

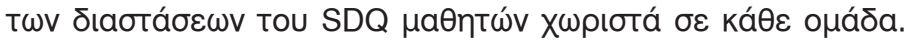

\begin{tabular}{|c|c|c|c|c|c|}
\hline \multirow{2}{*}{ Паıঠı́́ } & & \multicolumn{2}{|c|}{ 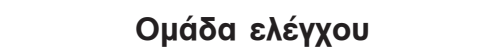 } & \multicolumn{2}{|c|}{ O^áठa EEA } \\
\hline & & 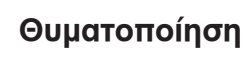 & Екфоßıбно́s & Өuнатопоі́nбп & Екфоßıбно́ \\
\hline \multirow[t]{2}{*}{ 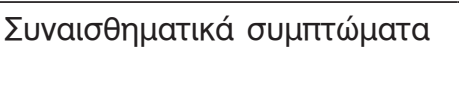 } & $r$ & 0,28 & 0,25 & 0,39 & 0,31 \\
\hline & $\mathrm{p}$ & 0,012 & 0,028 & $<0,001$ & 0,007 \\
\hline \multirow[t]{2}{*}{ 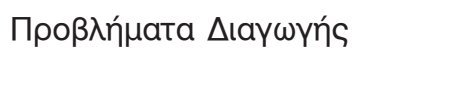 } & $r$ & 0,14 & 0,33 & 0,29 & 0,24 \\
\hline & $\mathrm{p}$ & 0,228 & 0,004 & 0,011 & 0,036 \\
\hline \multirow[t]{2}{*}{ ҮпєркІVптІко́тпта } & $r$ & 0,27 & 0,24 & 0,09 & 0,19 \\
\hline & $\mathrm{p}$ & 0,017 & 0,037 & 0,459 & 0,101 \\
\hline \multirow[t]{2}{*}{ 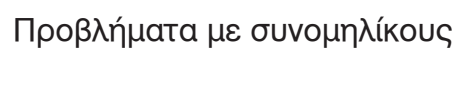 } & $\mathrm{r}$ & 0,49 & 0,37 & 0,27 & 0,09 \\
\hline & $\mathrm{p}$ & $<0,001$ & 0,001 & 0,020 & 0,442 \\
\hline \multirow[t]{2}{*}{ 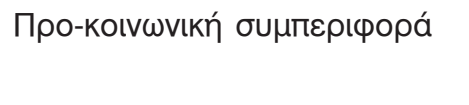 } & r & $-0,08$ & $-0,35$ & 0,10 & 0,04 \\
\hline & $\mathrm{p}$ & 0,461 & 0,002 & 0,395 & 0,735 \\
\hline \multirow[t]{2}{*}{ 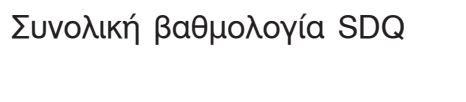 } & $\mathrm{r}$ & 0,46 & 0,44 & 0,38 & 0,29 \\
\hline & $\mathrm{p}$ & $<0,001$ & $<0,001$ & 0,001 & 0,02 \\
\hline
\end{tabular}

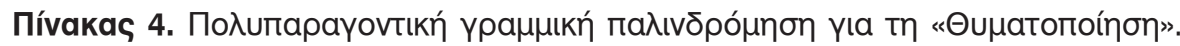

\begin{tabular}{|c|c|c|c|c|}
\hline & & $\beta^{*}$ & $\mathrm{SE}^{\star *}$ & $\mathbf{p}$ \\
\hline \multirow[t]{2}{*}{ 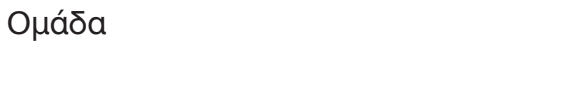 } & 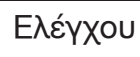 & $0,00+$ & & \\
\hline & EEA & 0,14 & 0,06 & 0,037 \\
\hline 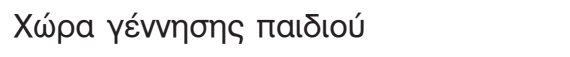 & & 4,77 & 2,07 & 0,023 \\
\hline 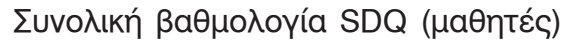 & & 0,34 & 0,07 & 0,001 \\
\hline
\end{tabular}

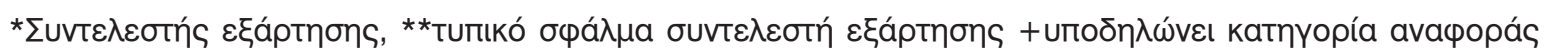




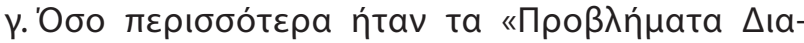

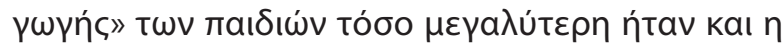

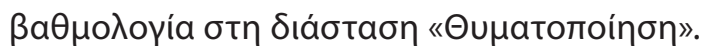

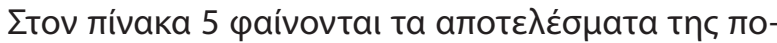

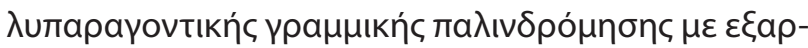

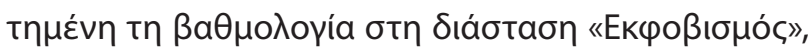

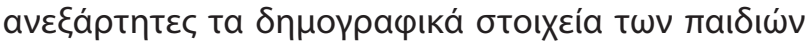

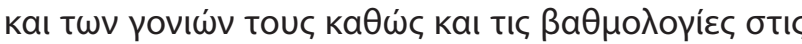

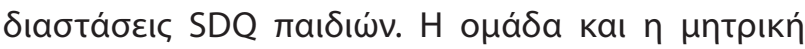

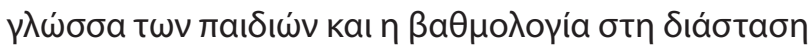

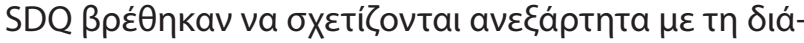

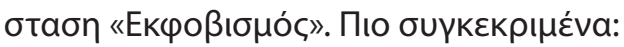

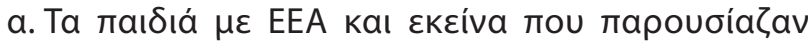

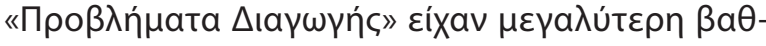

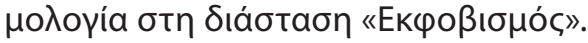

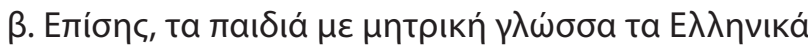

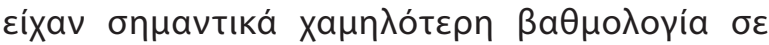

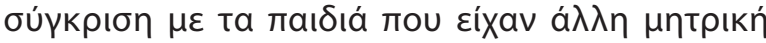

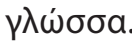

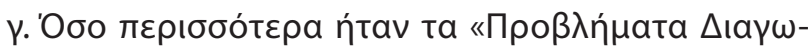

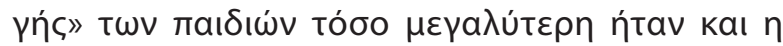

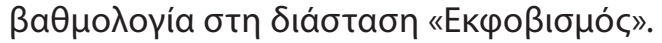

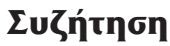

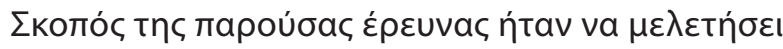

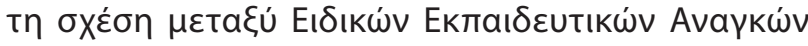

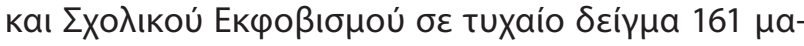

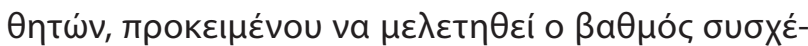

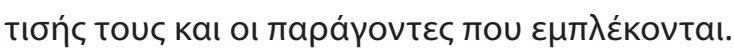

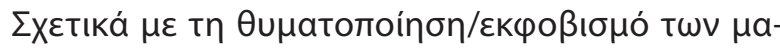

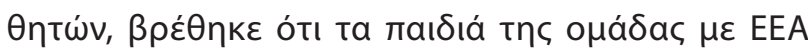

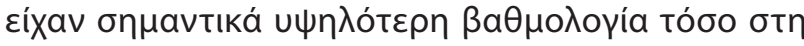

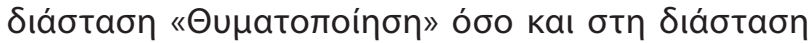

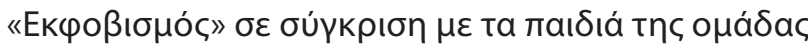

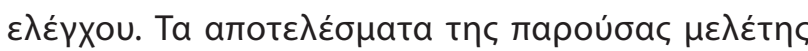

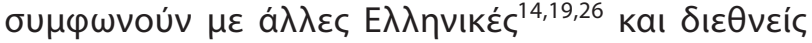

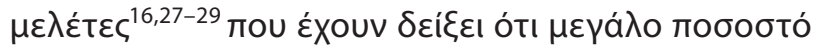

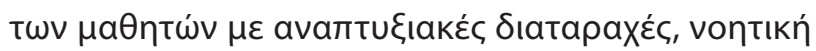

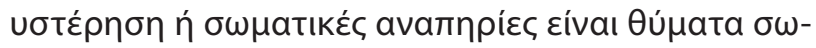

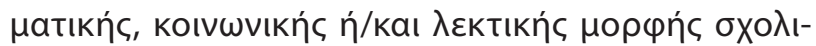

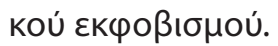

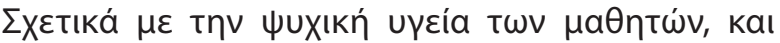

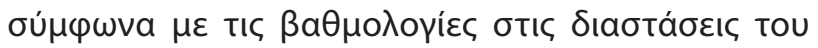

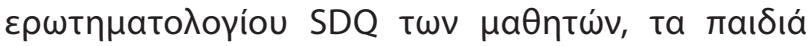

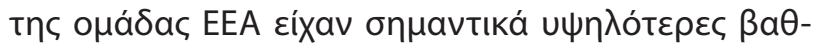

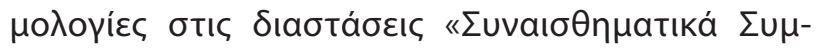

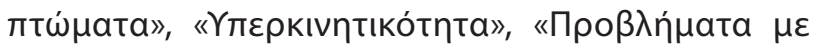

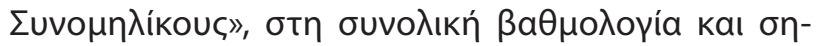

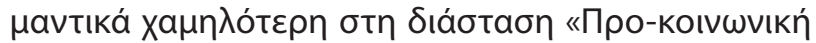

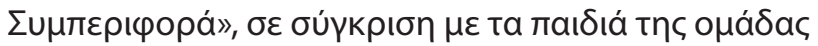
$\varepsilon \lambda \varepsilon ́ \gamma X O U$.

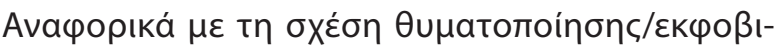

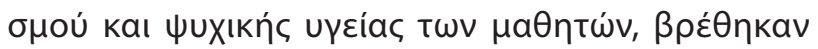

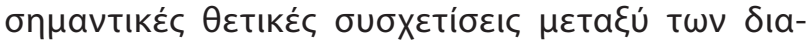

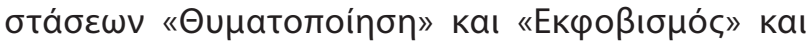

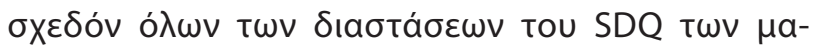

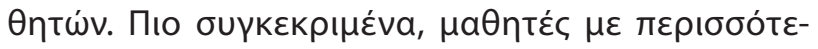

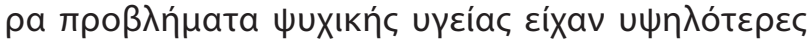

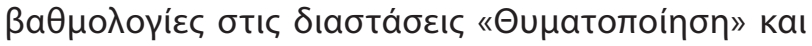

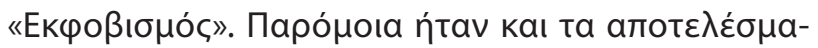

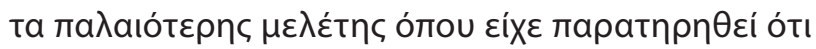

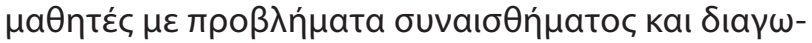

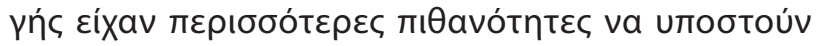
Өинатотоі́nбп. ${ }^{30-34}$

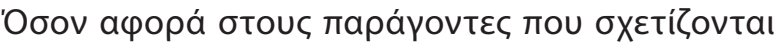

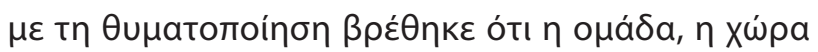

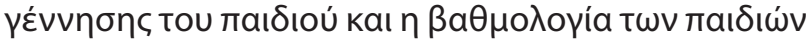

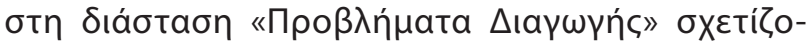

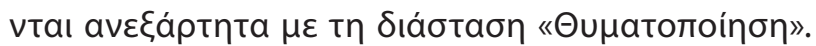

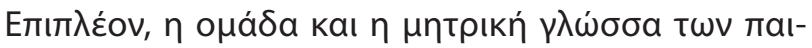

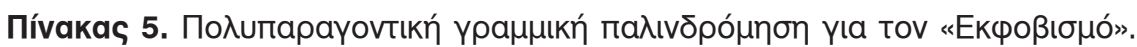

\begin{tabular}{|c|c|c|c|c|}
\hline & & $\beta^{*}$ & $\mathrm{SE}^{\star *}$ & $p$ \\
\hline \multirow[t]{2}{*}{ Oнáda } & 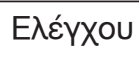 & $0,00+$ & & \\
\hline & EEA & 0,11 & 0,05 & 0,034 \\
\hline 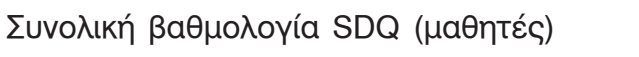 & & 0,18 & 0,05 & $<0,001$ \\
\hline \multirow{2}{*}{ 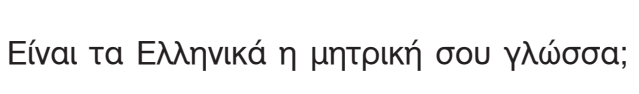 } & 'OXı & 0,00 & & \\
\hline & Nal & $-0,20$ & 0,06 & 0,002 \\
\hline
\end{tabular}

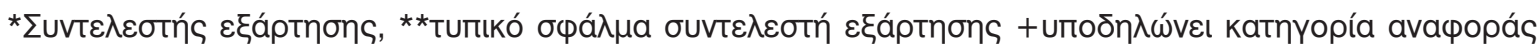




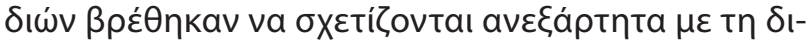

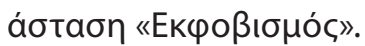

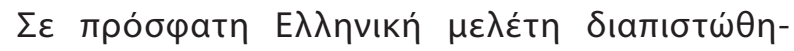

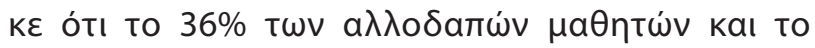

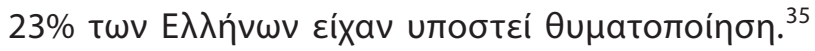

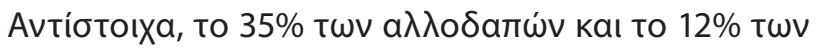

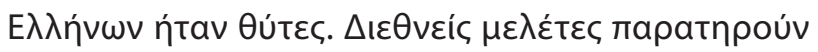

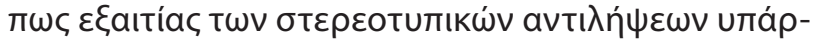

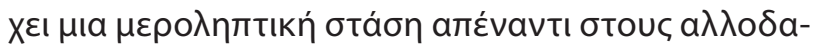

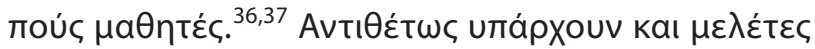

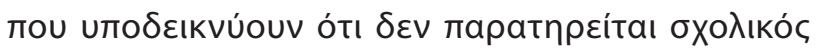

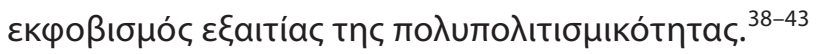

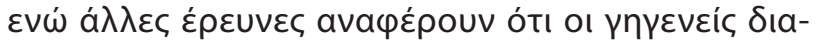

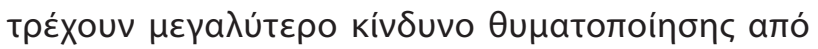

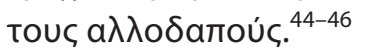

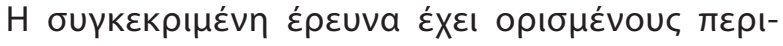

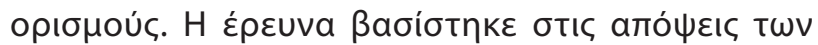

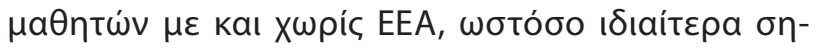

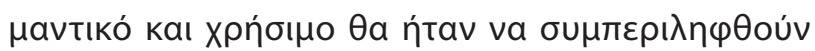

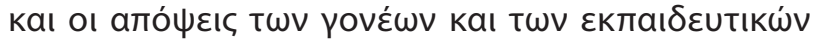

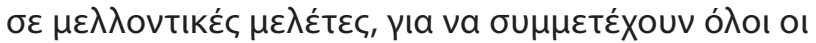

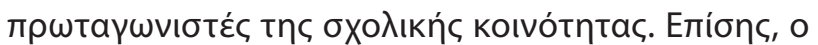

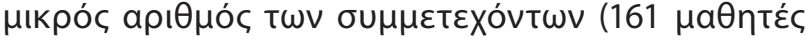

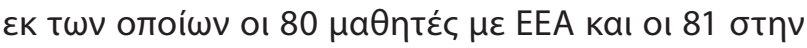

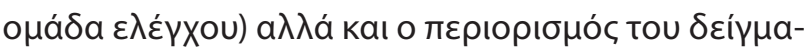

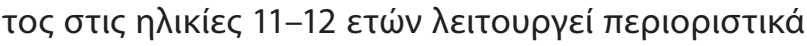

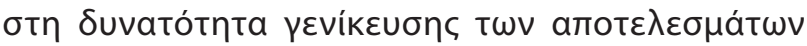

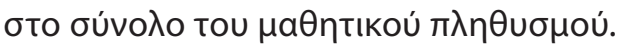

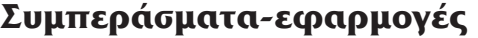

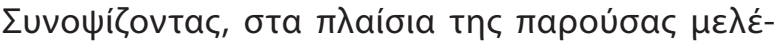

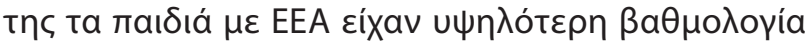

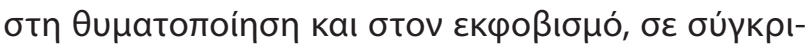

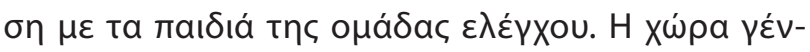

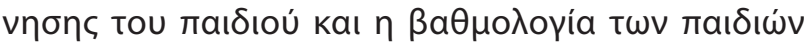

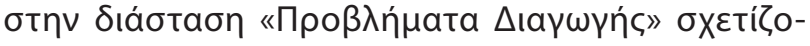

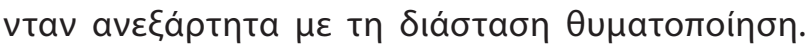

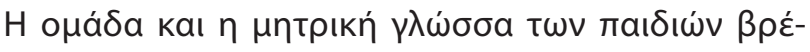

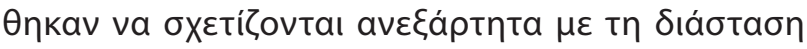

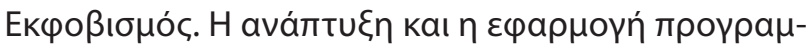

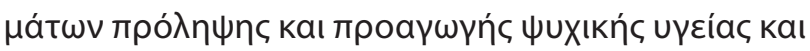

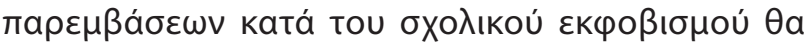

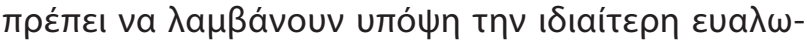

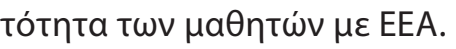

\title{
Bullying and the mental health of schoolchildren with special educational needs in primary education
}

\author{
A. Dasioti, ${ }^{1}$ G. Kolaitis ${ }^{2}$ \\ "'Mental Health Promotion-Prevention of Psychiatric Disorders", School of Medicine, \\ National and Kapodistrian University of Athens, Athens, \\ ${ }^{2}$ Department of Child Psychiatry, School of Medicine, National and Kapodistrian University of Athens, \\ "Aghia Sophia" Children's Hospital, Athens, Greece
}

Psychiatriki 2018, 29:149-159

The purpose of this study was to investigate the relationship between bullying and mental Health in schoolchildren with special educational needs (SEN). Random sampling was used to select two groups of children aged 11-12 years, attending the fifth and sixth grades of primary school and integration classes in urban and rural locations in Greece. The final sample comprised a group of 80 children with SEN and a control group of 81 children. The participants completed The Revised 
Olweus Bullying Victim Questionnaire and The Strengths and Difficulties Questionnaire (SDQ). The children in the SEN group scored significantly higher on both the "Victimization" dimension and the "Intimidation" dimension of the Olweus questionnaire than the children in the control group. On the SDQ, the SEN group scored significantly higher overall, and in the dimensions "Emotional Symptoms", "Hyperactivity" and "Peer Problems", and significantly lower in the dimension "Prosocial Behavior", indicating more mental health problems in comparison to the children in the control group. In addition, significant correlation was demonstrated between the dimensions "Victimization" and "Intimidation" of the Olweus questionnaire and almost all the SDQ dimensions. Multifactorial linear regression analysis showed that the SDQ scores were independently associated with the Olweus dimensions "Victimization" and "Bullying". In addition, higher scores in emotional symptoms and conduct problems were associated with higher scores in the dimensions 'Victimization' and Intimidation'. Higher scores in "Victimization" were recorded by children with SEN, but also by those who had not been born in Greece. Children with SEN and those with conduct problems scored higher in the dimension "Bullying" than children whose native language was not Greek. In conclusion, this study showed that in the fifth and sixth grades of primary school, a group of schoolchildren with SEN reported higher levels than a control group of victimization and bullying. These findings suggest that there is a great need for raising the awareness of this situation among the school staff through the provision of special training. Mental health preventive and promotive programs can enable school staff to identify students who are at higher risk of becoming victims of bullying and victimization. It is also recommended that children with SEN should be taught coping strategies against school bullying in the classroom and the wider school community.

Key words: School bullying, victimization, special educational needs, mental health.

\section{BıßAtoypacpía}

1. Olweus D. Bullying at School: Basic facts and effects of a school based intervention program. J Child Psychol Psychiatry 1994, 35:1171-1190, doi: 10.1111/j.1469-7610.1994.tb0 1229.x

2. Rigby K. New perspectives on bullying (ed) England. Jessica Kingsley publishers, 2002

3. Olweus D, Solberg ME. Prevalence estimation of school bullying with the Olweus Bully/Victim Questionnaire. J Aggress Behav 2003, 29:239-268, doi: 10.1002/ab.10047

4. Espelage DL. Leveraging school-based research to inform bullying prevention and policy. J Am Psychol 2016, 71:768775, doi: 10.1037/amp0000095

5. Venter E, Plessis E. Bullying in schools-The educator's role. J Koers 2012, 77:1-7, doi: 10.4102/koers.v77i1.34

6. World Health Organization. The World Health Report 2002: Reducing Risks, Promoting Healthy Life. World Health Organization, Geneva, 2002

7. Gilbert R, Widom CS, Browne K, Fergusson D, Webb E, Janson S. Burden and consequences of child maltreatment in high-income countries. Lancet 2009, 373:68-81, doi: $10.1016 /$ S0140-6736(08)61706-7
8. Copeland WE, Wolke D, Angold A, Costello EJ. Adult psychiatric outcomes of bullying and being bullied by peers in childhood and adolescence. JAMA Psychiatry 2013, 70:419-426, doi: 10.1001/jamapsychiatry.2013.504

9. Takizawa R, Mau.ghan B, Arseneault L. Adult health outcomes of childhood bullying victimization: evidence from a five-decade longitudinal British birth cohort. Am J Psychiatry 2014, 171:777-784, doi: 10.1176/appi.ajp.2014.13 101401

10. Bradshaw CP, Sawyer AL, O'Brennan LM. Bullying and peer victimization at school: perceptual differences between students and school staff. J Sch Psychol Rev 2007, 36:361-382

11. Nansel TR, Overpeck M, Pilla RS, Ruan WJ, Simons-Morton B, Scheidt P. Bullying behaviors among US youth: prevalence and association with psychosocial adjustment. JAMA 2001, 285:2094-2100, doi: 10.1001/jama.285.16.2094

12. Spriggs AL, lannotti RJ, Nansel TR, Haynie DL. Adolescent bullying involvement and perceived family, peer and school relations: commonalities and differences across race/ethnicity. J Adolesc Health 2007, 41:283-293, doi: 10.1016/j.jado health.2007.04.009 
13. Mayes SD, Calhoun SL, Baweja R, Mahr F. Maternal ratings of bullying and victimization: differences in frequencies between psychiatric diagnoses in a large sample of children. J Psychol Rep Disabil Trauma 2015, 116:710-722, doi: 10.2466/16.PR0.116k30w8

14. Chatziiona-Paradisioti A. Emotional and behavioral disorders that cause school bullying in children and teenagers aged 11-14 in Cyprus (dissertation). National Archives of Doctoral. Greece, 2015, 1-320

15. Zablotsky B, Bradshaw CP, Anderson CM, Law P. Risk factors for bullying among children with autism spectrum disorders. J Autism 2014, 18:419-427, doi: 10.1177/1362361313477920

16. Christensen LL, Fraynt RJ, Neece CL, Baker BL. Bullying adolescents with intellectual disability. J Ment Health Res Intellect Disabil 2012, 5:49-65, doi: 10.1080/19315864.2011.637660

17. Berg KL, Shiu CS, Msall ME, Acharya K. Victimization and depression among youth with disabilities in the US child welfare system. J Child: Care, Health Dev 2015, 41:989-999, doi: $10.1111 /$ cch. 12233

18. Humphrey JL, Storch EA, Geffken GR. Peer victimization in children with attention-deficit hyperactivity disorder. J Child Health Care 2007, 11:248-260, doi: 10.1177/1367493507079571

19. Andreou E, Didaskalou E, Vlachou A. Bully/victim problems among Greek pupils with special educational needs: associations with loneliness and self-efficacy for peer interactions. J Res Special Educ Needs 2015, 15:235-246, doi:10.1111/1471-3802.12028

20. Rose C, Espelage DL, Aragon SR, Elliott J. "Bullying and victimization among students in special education and general education curricula". J Exceptionality Educ Int 2011, 21:2-14

21. Blood G, Boyle MP, Blood IM, Nalesnik GR. Bullying in children who stutter: speech-language pathologists' perceptions and intervention strategies. J Fluency Disord 2010, 35:92-109, doi: 10.1016/j.jfludis.2010.03.003

22. Olweus D. The revised Olweus bully/victim questionnaire. Mimeo, Research Center for Health Promotion (HEMIL Center). University of Bergen, Norway, 1996

23. Breivik K, Olweus D. An item response theory analysis of the Olweus Bullying scale. J Aggress Behav 2014, 41:1-13, doi: 10.1002/ab.21571

24. Goodman R. The extended version of the strengths and difficulties questionnaire as a guide to child psychiatric caseness and consequent burden. $J$ Child Psychol Psychiatry 1999, 40:791-799

25. Giannakopoulos G, Tzavara KC, Dimitrakaki C, Kolaitis G, Rotsika V, Tountas $Y$. The factor structure of the Strengths and Difficulties Questionnaire (SDQ) in Greek adolescents. J Ann Gen Psychiatry 2009, 8:1-7, doi: 10.1186/1744-859X8-20

26. Kokkiadis M, Kourkoutas H. Bullying/victimization, selfesteem and emotional difficulties in children with and without
SEN. Department of Preschool Education. Scient Yearbook 2016, 9:89-128

27. Ttofi MM, Farrington DP, Lösel F, Loeber R. Do the victims of school bullies tend to become depressed later in life? A systematic review and meta-analysis of longitudinal studies. J Aggr 2011, 3:63-73, doi: 10.1108/17596591111132873

28. Reiter S, Lapidot-Lefler N. Bullying among special education students with intellectual disabilities: differences in social adjustment and social skills. J Am Assoc Intell Dev Dis 2007, 45: 174-181, doi: 10.1352/1934-9556(2007)45[174:BASESW ]2.0.CO;2

29. Berg KL, Shiu CS, Msall ME, Acharya K. Victimization and depression among youth with disabilities in the US child welfare system. J Child: Care, Health Dev 2015, 41:989-999, doi: $10.1111 /$ cch.12233

30. Fekkes M, Frans IM, Pijpers A, Fredriks M, Vogels T, VerlooveVanhorick SP. Do Bullied Children Get III, or Do III Children Get Bullied? A Prospective Cohort Study on the Relationship Between Bullying and Health-Related Symptoms. J Pediatr 2006, 117

31. Card NA, Isaacs J, Hodges EVE. Multiple contextual levels of risk for peer victimization: a review with implications for prevention and intervention efforts. In: Miller TW (ed) School Violence and Primary Prevention. Springer, New York, 2008:125-153, doi: 10.1007/978-0-387-77119-9_7

32. Macintosh K, Dissanayake C. Social skills and problem behaviours in school-aged children with high-functioning autism and Asperger's disorder. J Autism Dev Disord 2006, 36:1065-1076, doi:10.1007/s10803-006-0139-5

33. Wright MF. School bullying and students with intellectual disabilities, handbook of research on diagnosing. In: Gopalan, Rejani Thudalikunnil (ed) Handbook of research on diagnosing, treating and managing intellectual disabilities. India, 2016:1-496, doi:10.4018/978-1-5225-(X)89-6.ch003

34. Hoza B, Mrug S, Gerdes AC, Hinshaw SP, Bukowski WM, Gold JA, et al. "What Aspects of Peer relationships Are Impaired in Children with Attention-deficit/Hyperactivity Disorder?". J Consult Clin Psychol 2005, 73: 411-423, doi: 10.1037/0022-006X.73.3.411

35. Houndoumadi H, Pateraki L, Doanidou M. Violence in schools: the response in Europe (ed) Peter K Smith, London, England, 2003:169-183

36. Cobia DC, Carney JS. Creating a culture of tolerance in schools: Everyday actions to prevent hate-motivated violent incidents. J Sch Violence 2002, 1:87-104

37. Pagani C, Robustelli F, Martinelli C. School, cultural diversity, multiculturalism and contact. J Intercult Educ 2011, 22:337349, doi: 10.1080/14675986.2011.617427

38. Psalti A, Konstantinou K. The phenomenon of bullying in secondary schools: The influence of gender and ethnicity of cultural origin. Psychology 2007, 14:329-345 
39. Larochette AC, Murphy AN, Craig WM. Racial bullying and victimization in Canadian school aged children individual and school level effects. J Sch Psychol Inter 2010, 31:389-408

40. Boulton MJ. Patterns of bully/victim problems in mixed race groups of children. J Soc Dev 1995, 4:277-293, doi: 10.1111/j. 1467-9507.1995.tb00066.x

41. Eslea M, Mukhtar K. Bullying and racism among Asian schoolchildren in Britain. J Educ Res 2000, 42:207-217, doi: $10.1080 / 001318800363845$

42. Mckenney KS, Pepler D, Craig WM, Connolly J. Peer victimization and psychosocial adjustment: The experiences of Canadian immigrant youth. Electron J Res Educ Psychol 2006, 9:230-264

43. Fandrem H, Strohmeier D, Roland E. Bullying and victimization among Native and immigrant adolescents in Norway: the role of proactive and reactive aggressiveness. J Early Adolesc 2009, 29:898-923, doi: 10.1177/0272431609332935

44. Hanish LD, Guerra NG. The roles of ethnicity and school context in predicting children's victimization by peers. $\mathrm{Am}$ J Community Psychol 2000, 28:201-223, doi: 10.1023/A: 1005187201519
45. Strohmeier D, Spiel C. Immigrant children in Austria: Aggressive behavior and friendship patterns in multicultural school classes. J Appl Sch Psychol 2003, 19:99-116, doi: $10.1300 / J 008$ v19n02_07

46. Strohmeier D, Spiel C, Gradinger P. Social relationships in multicultural schools: Bullying and victimization. Eur J Dev Psychol 2008, 5:262-285, doi: 10.1080/1740 5620701556664

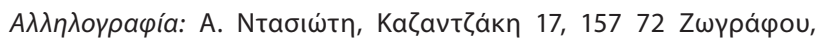
AӨńva, e-mail: athina_das@hotmail.com 


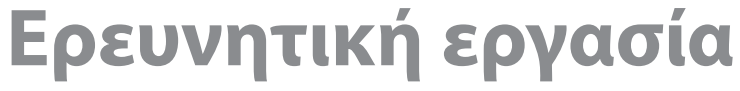 Research article
}

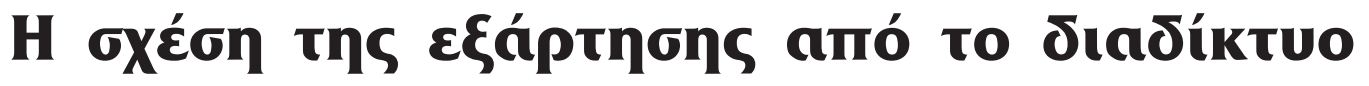

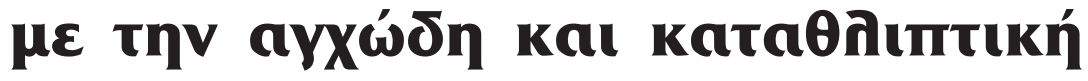

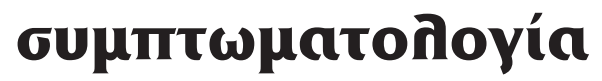

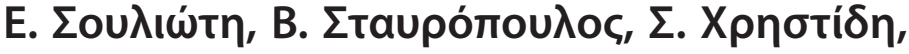

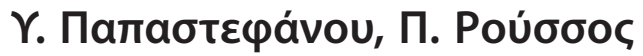

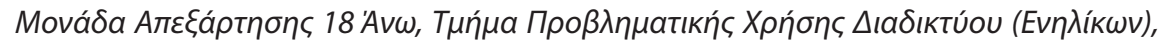

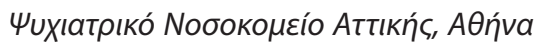 \\ Uuxıatpıкń 2018, 29:160-171
}

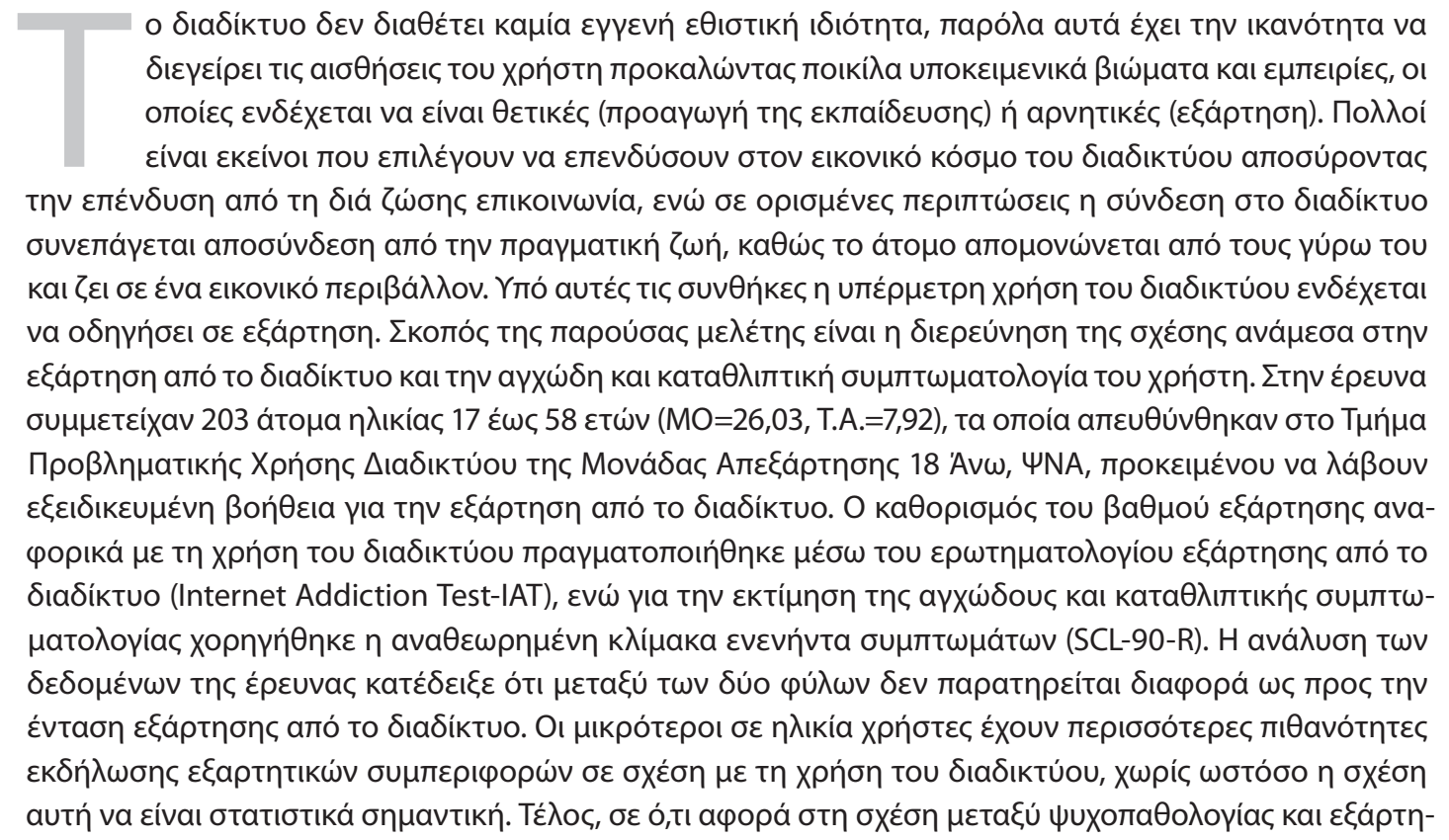




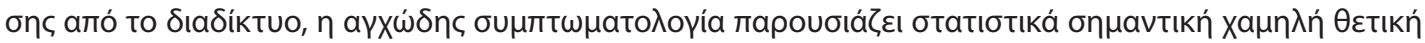

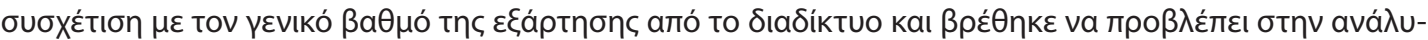

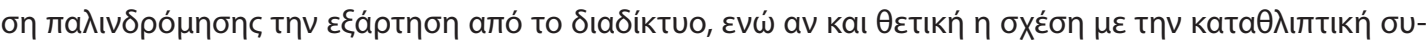

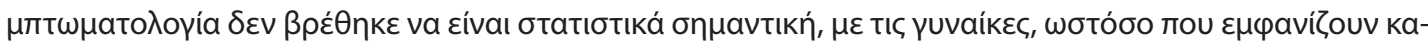

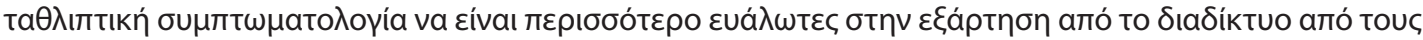

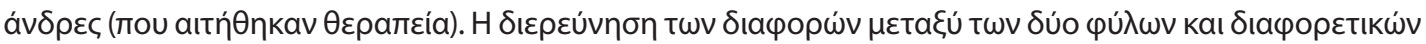

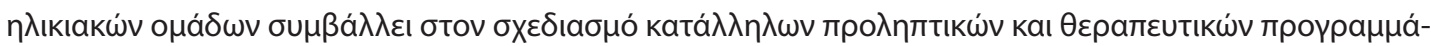

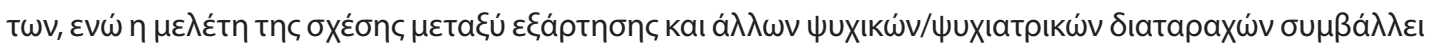

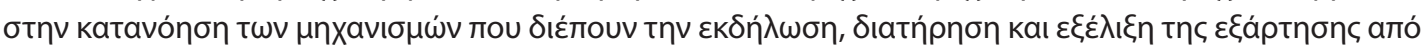

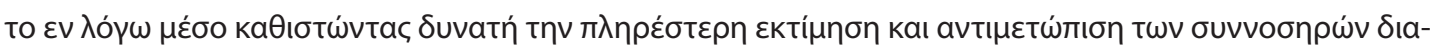

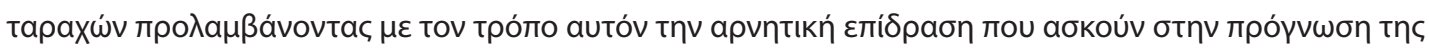

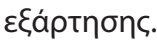

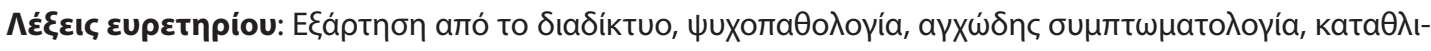

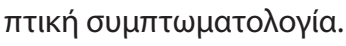

\section{Eıăywyń}

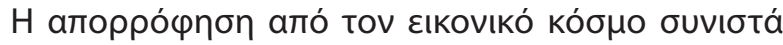

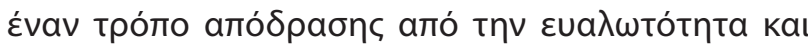

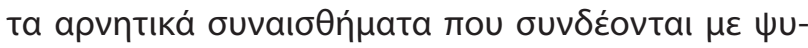

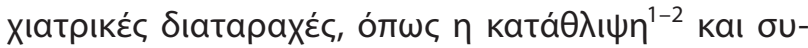

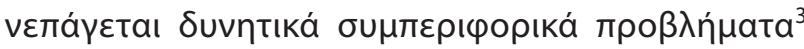

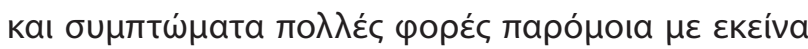

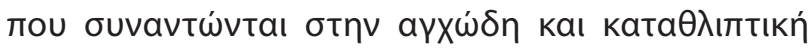

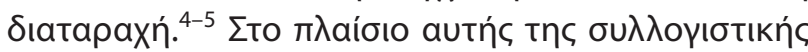

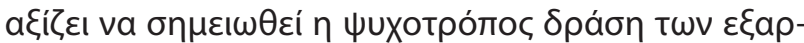

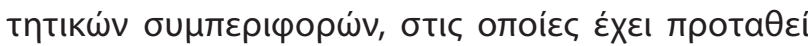

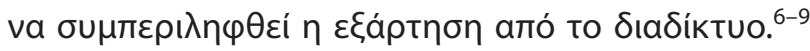

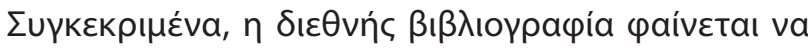

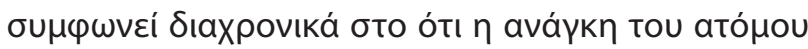

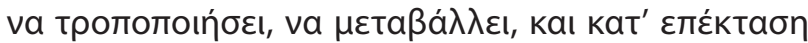

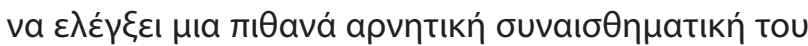

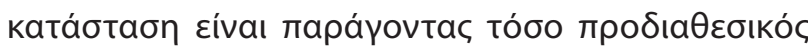

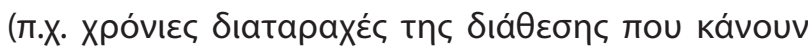

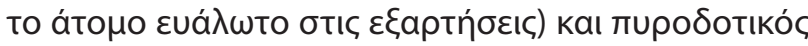

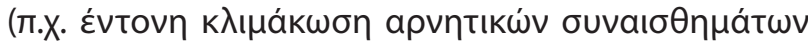

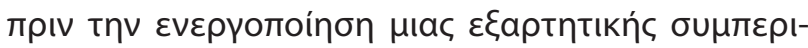

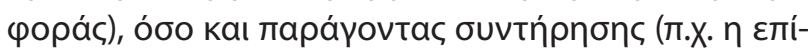

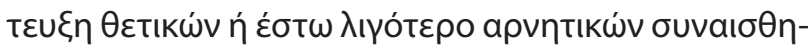

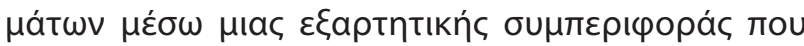

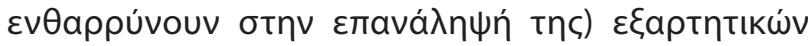

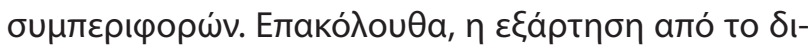

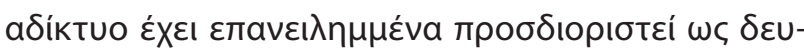

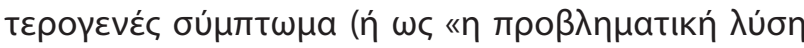

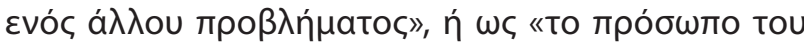

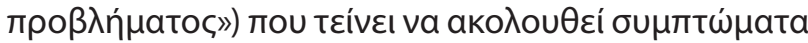

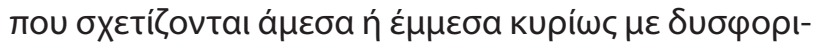

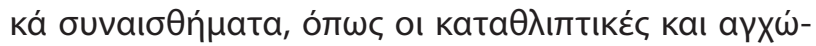

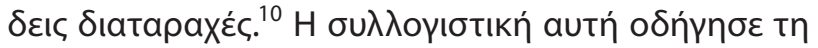

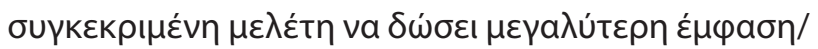

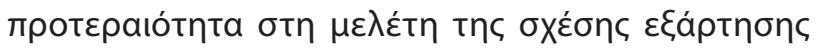

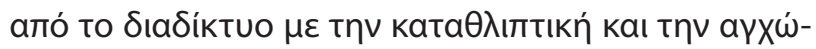

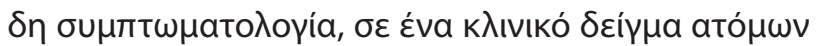

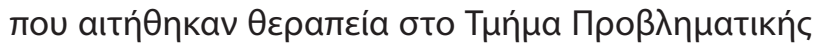

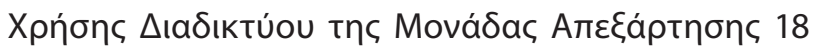

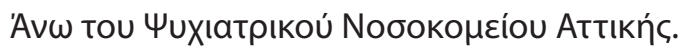

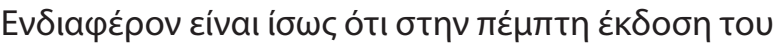

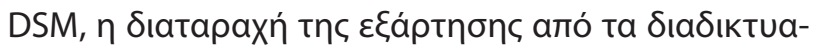

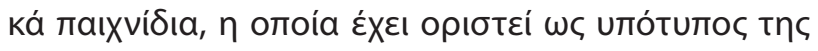

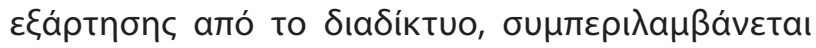

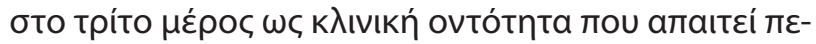

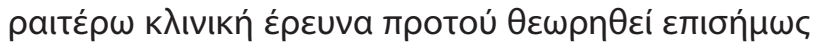

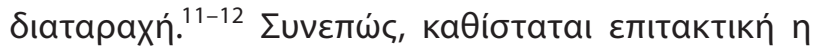

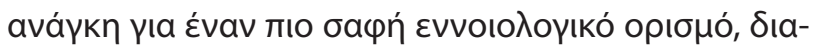

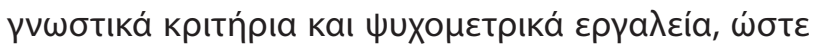

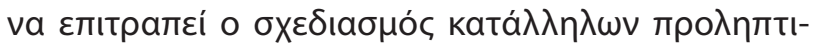

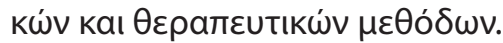

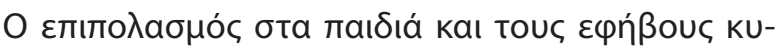

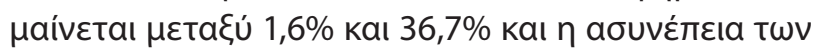

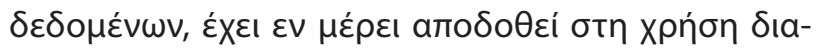

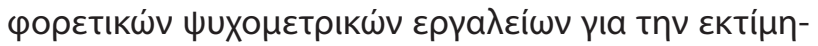

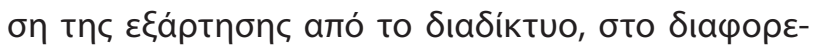

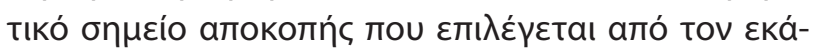

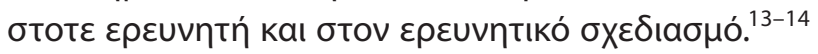

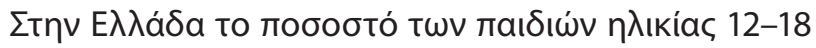

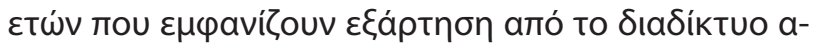

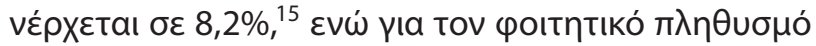




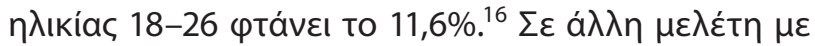

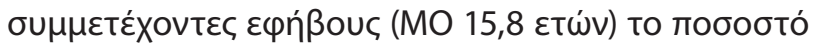

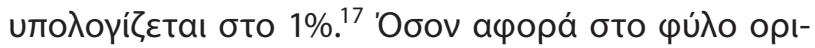

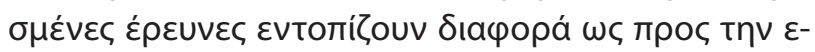

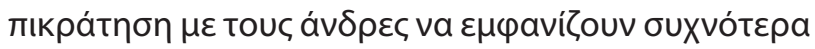

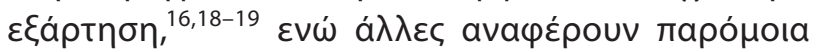

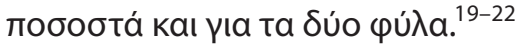

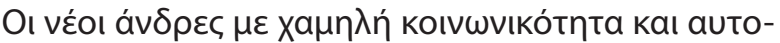

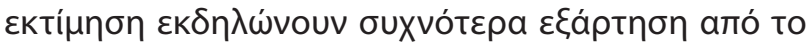

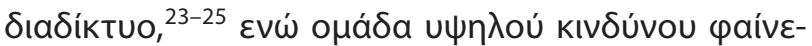

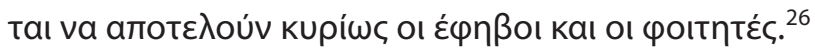

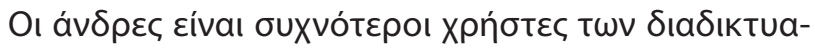

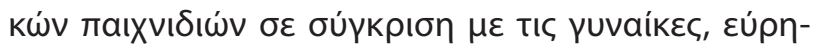

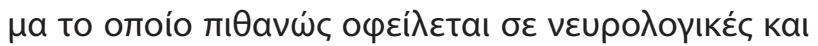

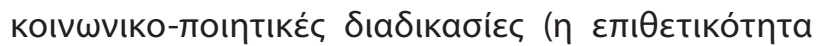

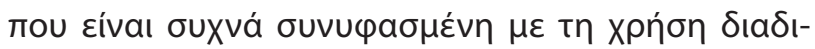

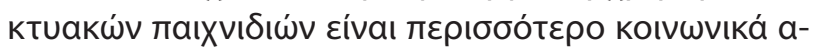

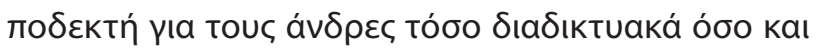

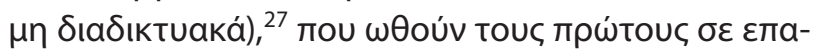

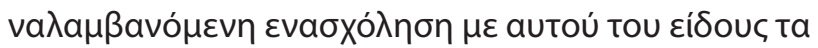

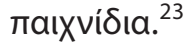

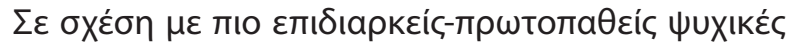

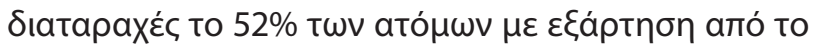

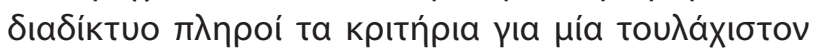

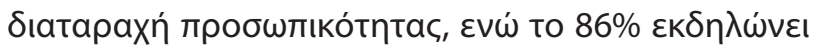

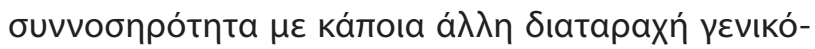

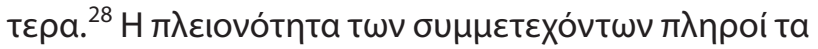

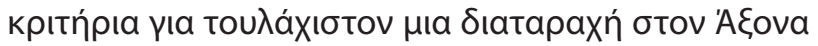

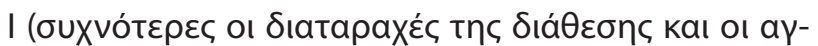

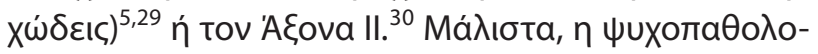

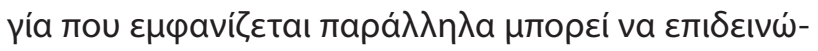

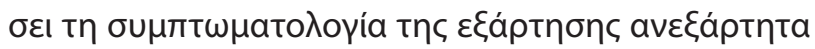

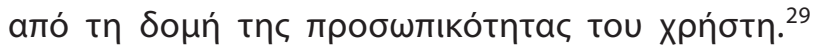

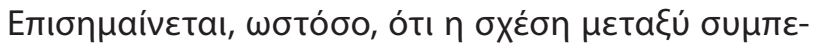

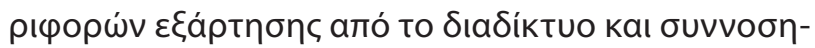

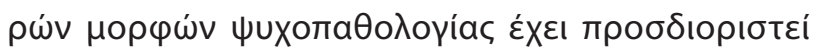

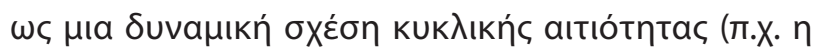

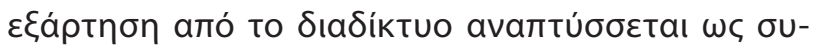

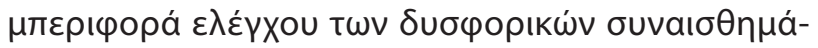

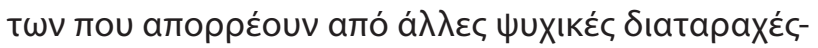

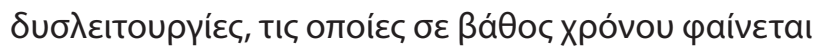
va $\varepsilon \pi ı \delta \varepsilon ı v \omega ́ v \varepsilon ı .{ }^{6}$

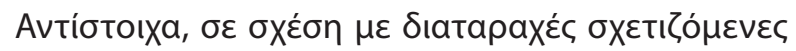

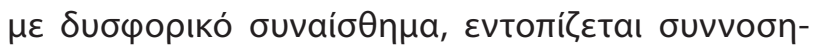

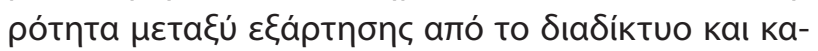

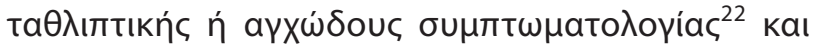

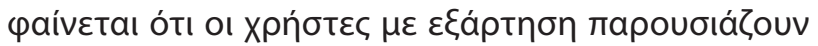

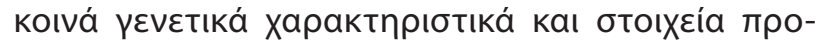

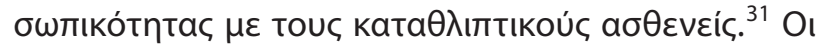

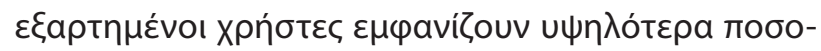

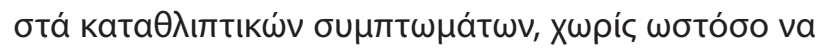

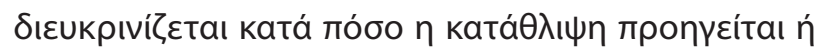

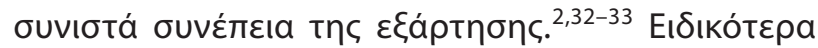

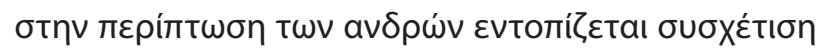

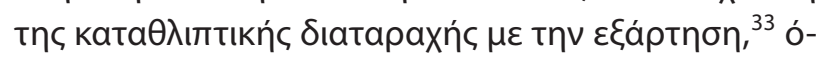

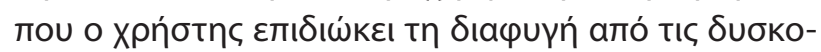

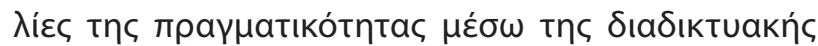

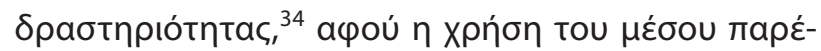

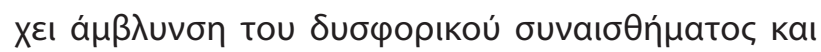

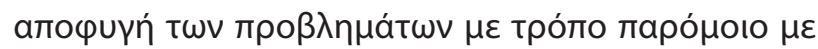

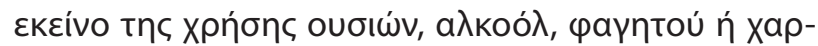

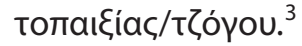

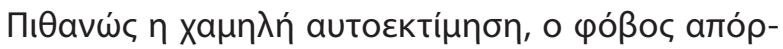

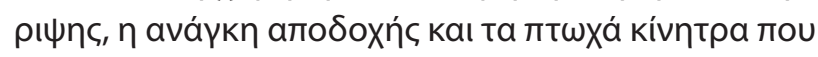

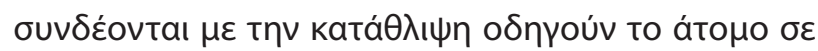

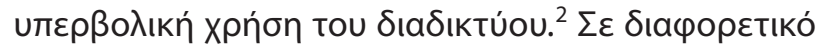

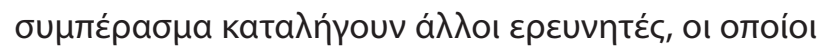

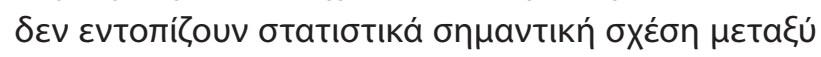

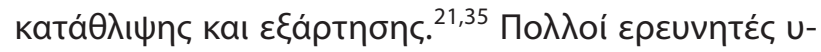

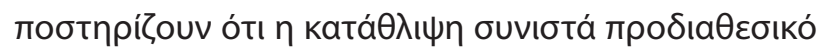

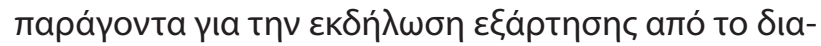

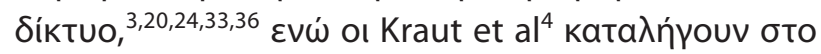

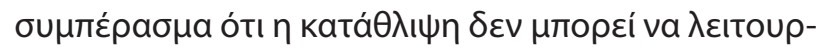

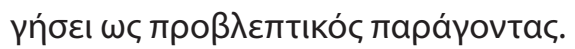

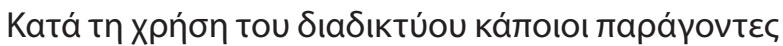

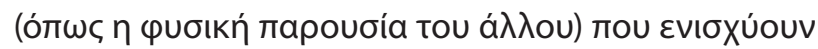

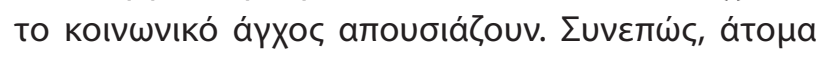

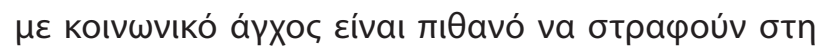

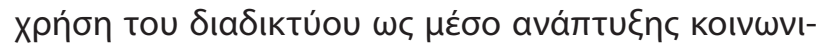

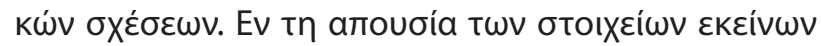

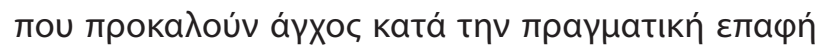

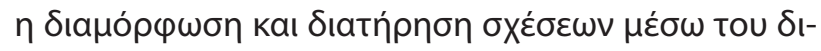

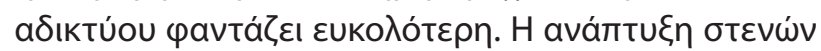

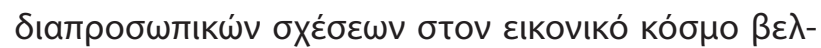

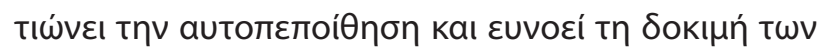

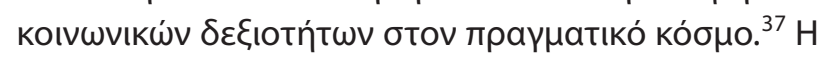

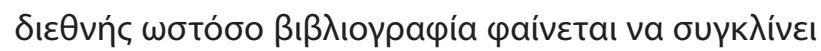

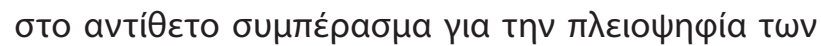

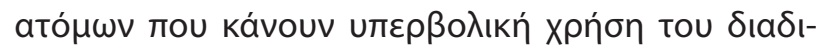

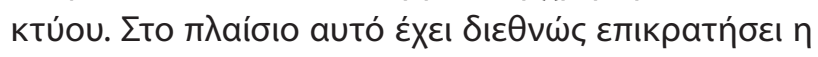

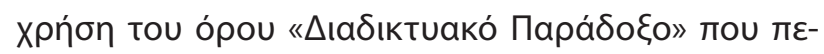

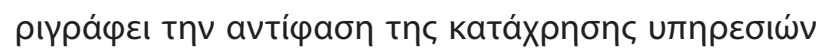

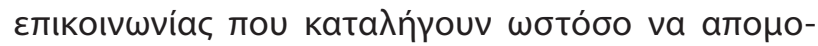




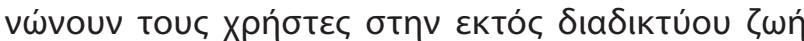
Touc. ${ }^{38}$

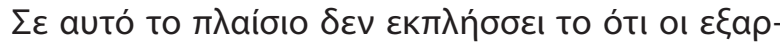

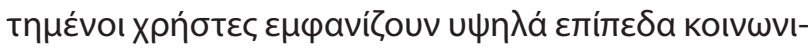

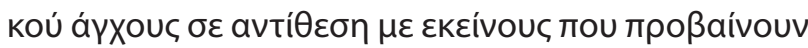

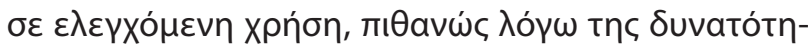

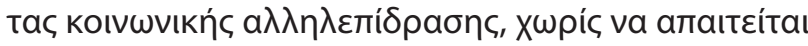

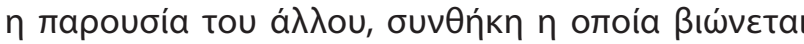

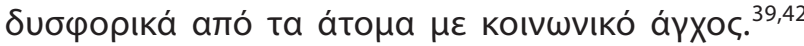

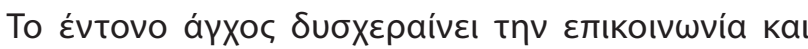

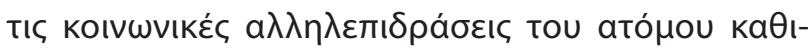

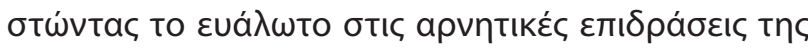

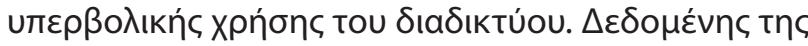

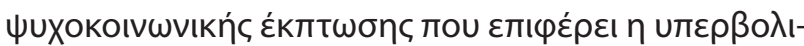

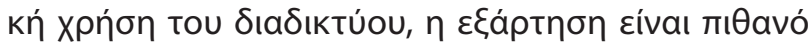
va $\varepsilon \pi$

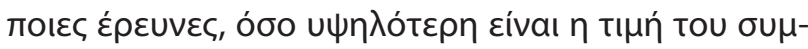

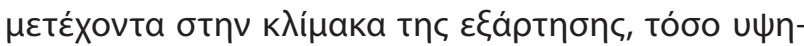

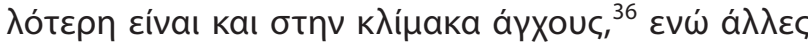

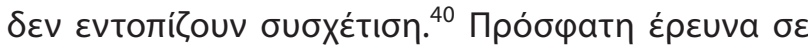

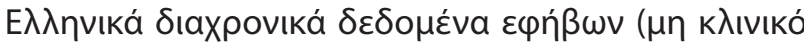

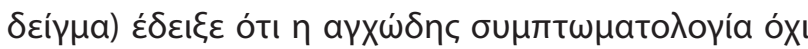

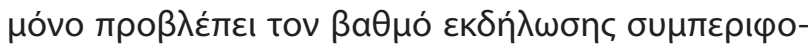

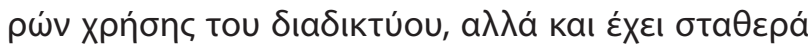

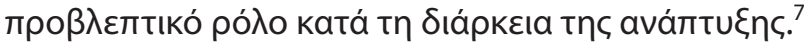

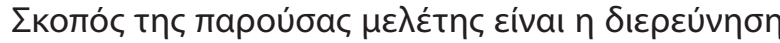

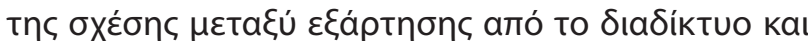

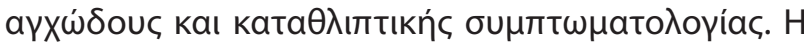

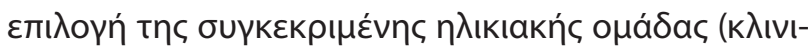

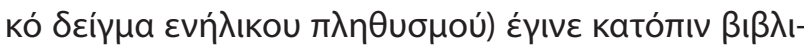

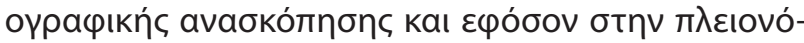

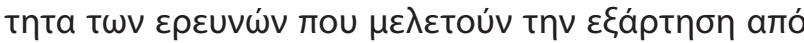

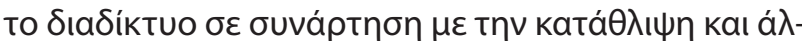

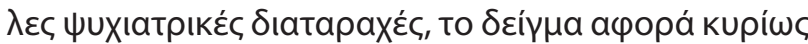

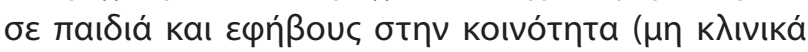

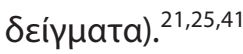

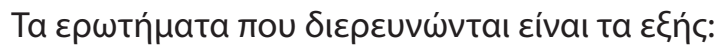

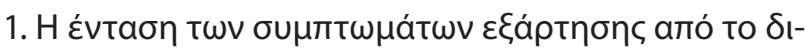

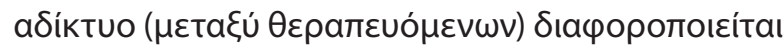

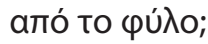

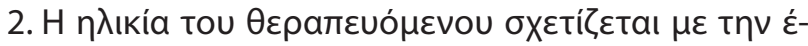

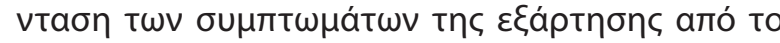

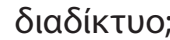

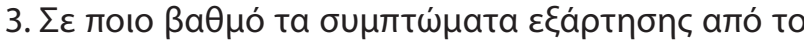

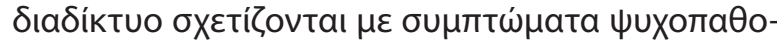

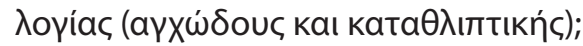

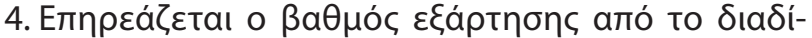

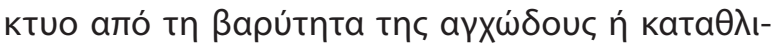

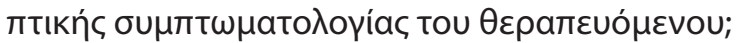

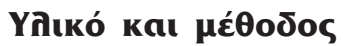

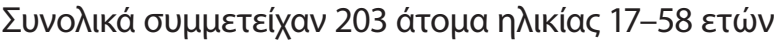

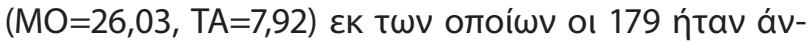

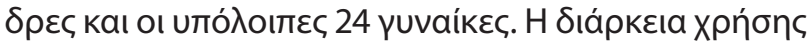

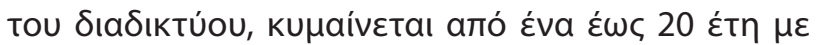

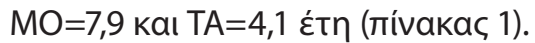

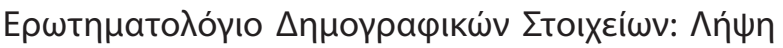

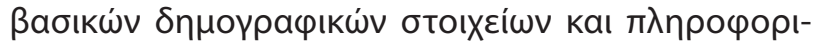

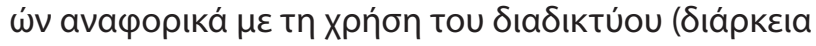

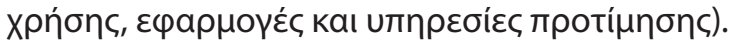

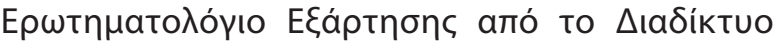

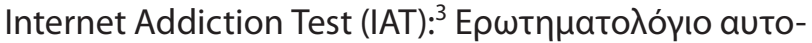

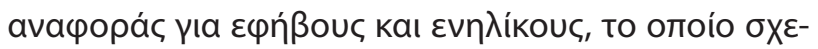

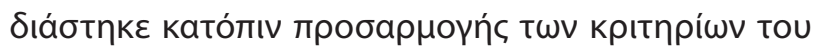

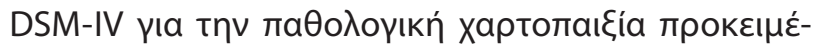

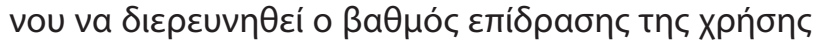

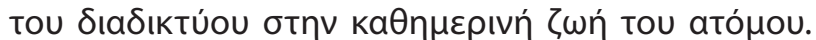

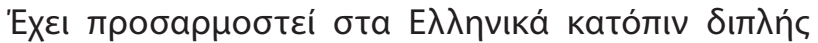

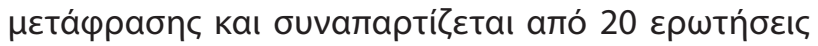

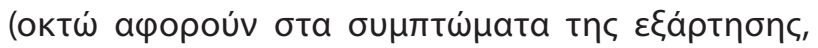

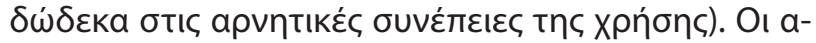

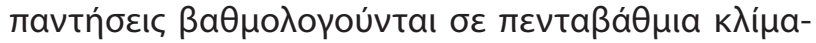

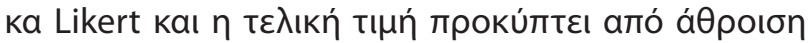

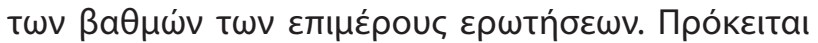

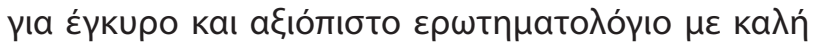

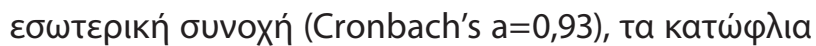

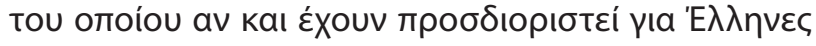

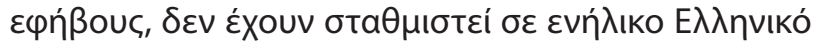
$\pi \lambda \eta \theta u \sigma \mu$. $^{42-43}$

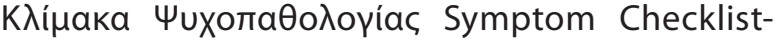

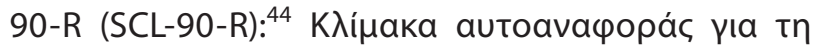

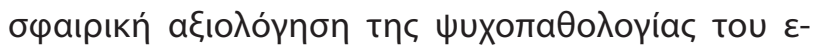

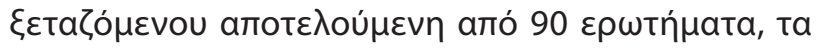

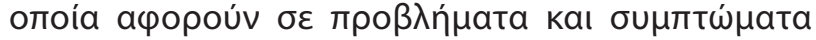

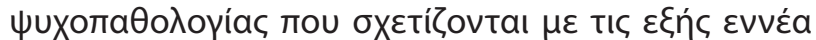

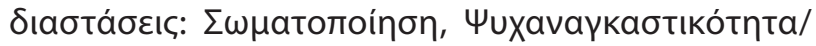

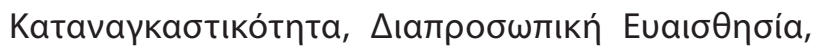

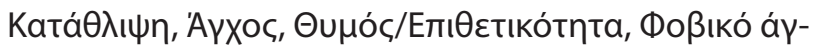

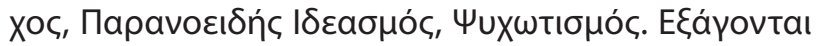

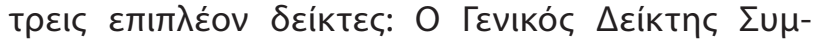

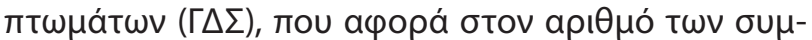

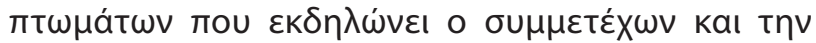




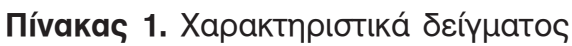

\begin{tabular}{|c|c|c|c|c|c|}
\hline & & & \multicolumn{2}{|c|}{ Фúdo } & \multirow{2}{*}{ ¿uvoגıкó } \\
\hline & & & Гuvaíka & 'Avסра؟ & \\
\hline \multirow{10}{*}{ 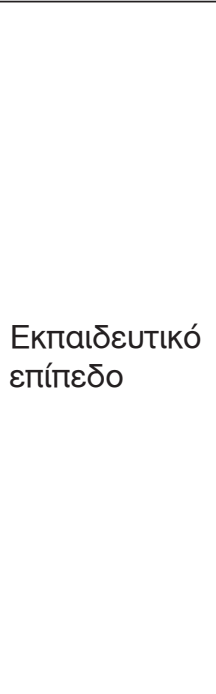 } & \multirow[b]{2}{*}{ Гuнváбıı } & $\mathrm{n}$ & 1 & 8 & 9 \\
\hline & & 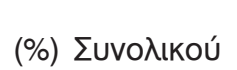 & $0,6 \%$ & $4,7 \%$ & $5,2 \%$ \\
\hline & \multirow{2}{*}{ 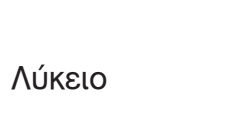 } & $\mathrm{n}$ & 11 & 86 & 97 \\
\hline & & 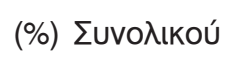 & $6,4 \%$ & $50,0 \%$ & $56,4 \%$ \\
\hline & \multirow{2}{*}{ IEK/Ko入ع́үı૦ } & $\mathrm{n}$ & 5 & 14 & 19 \\
\hline & & (\%) $\Sigma$ uvoגıкоú & $2,9 \%$ & $8,1 \%$ & $11,0 \%$ \\
\hline & \multirow[b]{2}{*}{ TEI/AEI } & $\mathrm{n}$ & 3 & 34 & 37 \\
\hline & & (\%) $\Sigma$ Uvoגıкoú & $1,7 \%$ & $19,8 \%$ & $21,5 \%$ \\
\hline & \multirow[b]{2}{*}{ MeтаптuXıакá } & $\mathrm{n}$ & 1 & 9 & 10 \\
\hline & & 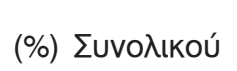 & $0,6 \%$ & $5,2 \%$ & $5,8 \%$ \\
\hline \multirow{2}{*}{\multicolumn{2}{|c|}{ 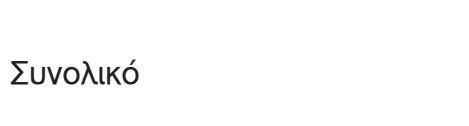 }} & $\mathrm{nN}$ & 21 & 151 & 172 \\
\hline & & (\%) $\Sigma$ uvoגıкоú & $12,2 \%$ & $87,8 \%$ & $100,0 \%$ \\
\hline \multicolumn{2}{|l|}{ H入ıкía } & & $\begin{array}{l}\mathrm{MO}=27,9 \\
\mathrm{TA}=10,55\end{array}$ & $\begin{array}{l}\mathrm{MO}=25,77 \\
\mathrm{TA}=7,48\end{array}$ & $\begin{array}{l}\mathrm{MO}=26,02 \\
\mathrm{TA}=7,90\end{array}$ \\
\hline \multicolumn{2}{|c|}{ 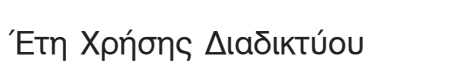 } & & $\begin{array}{l}\mathrm{MO}=5,37 \\
\mathrm{TA}=3,47\end{array}$ & $\begin{array}{l}\mathrm{MO}=8,32 \\
\mathrm{TA}=4,11\end{array}$ & $\begin{array}{l}\mathrm{MO}=7,93 \\
\mathrm{TA}=4,15\end{array}$ \\
\hline
\end{tabular}

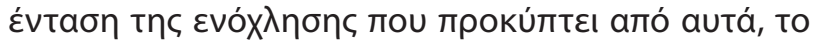

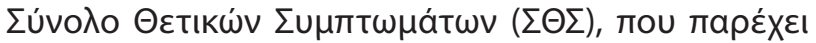

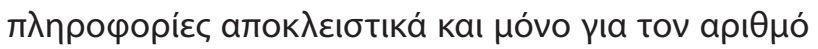

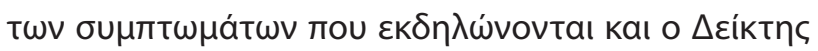

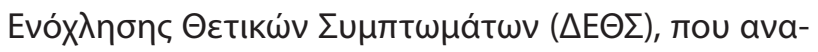

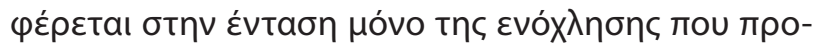

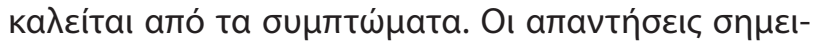

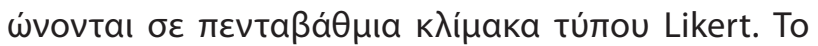

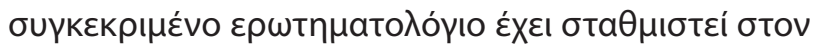

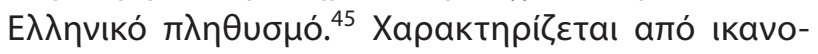

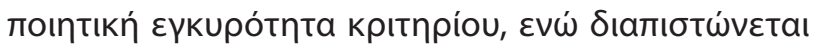

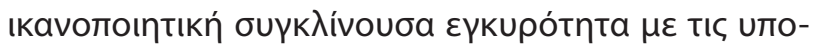

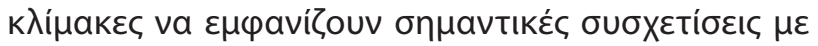

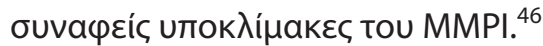

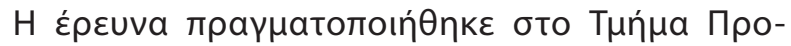

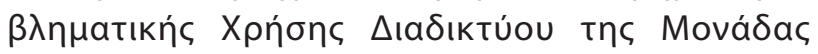

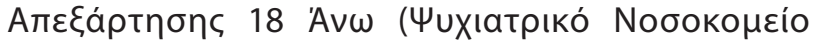

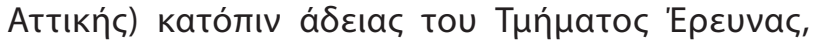

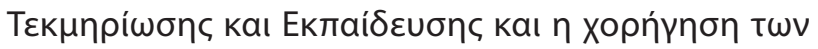

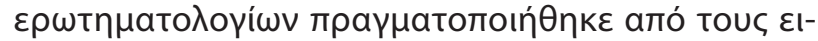

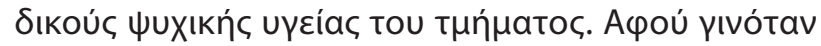

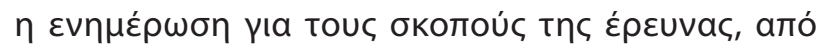

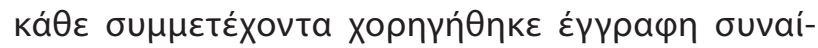

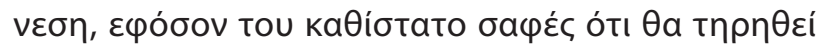

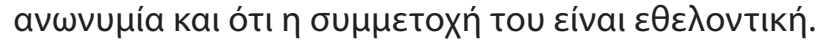

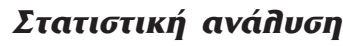

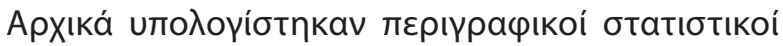

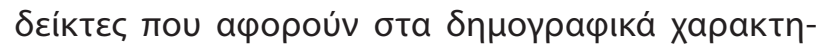

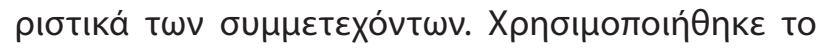

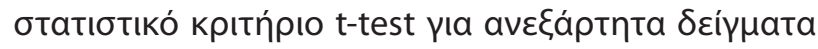

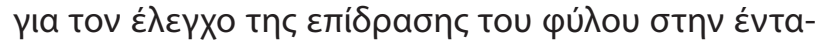

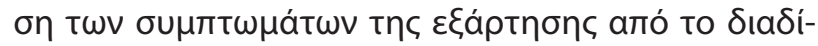

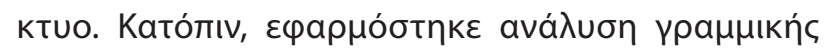

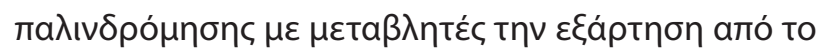

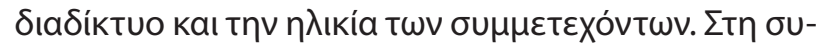

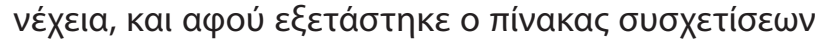

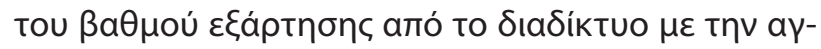




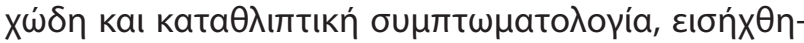

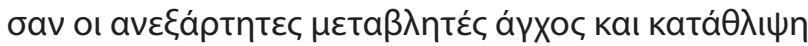

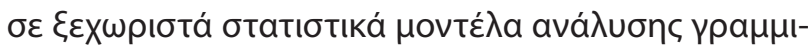

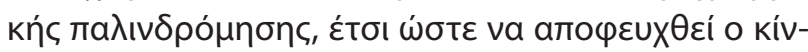

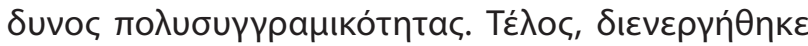

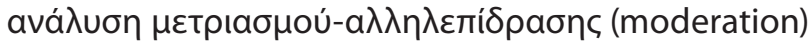

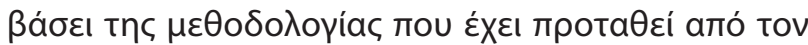

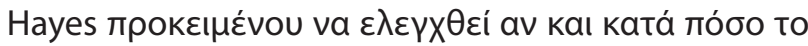

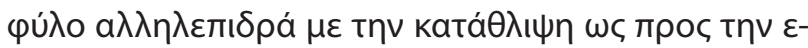

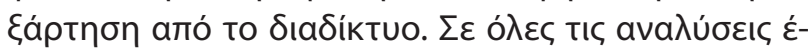

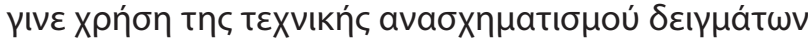

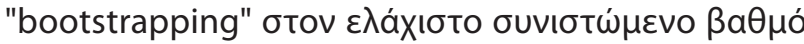

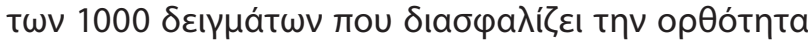

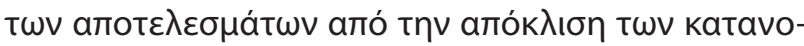

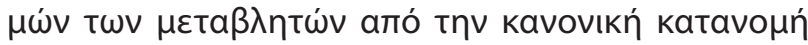

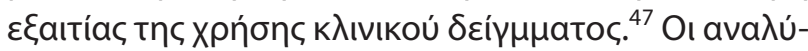

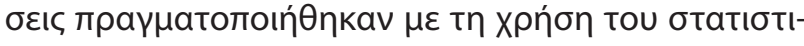

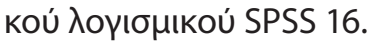

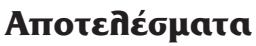

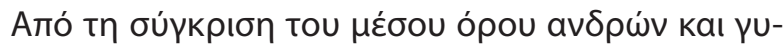

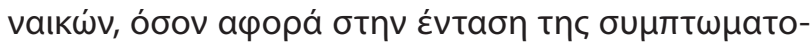

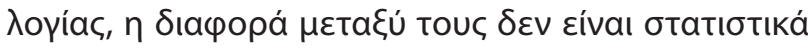

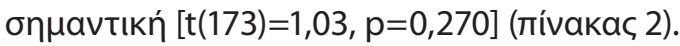

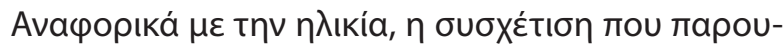

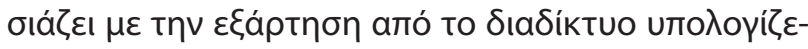

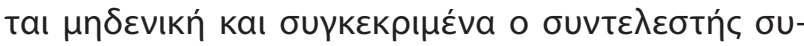

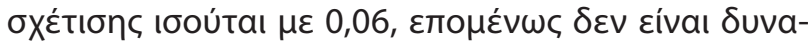

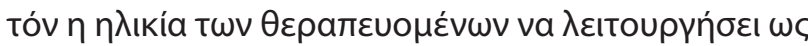

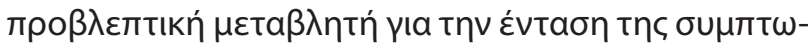

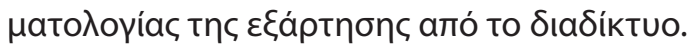

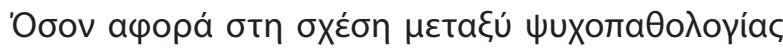

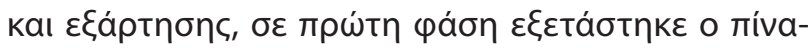

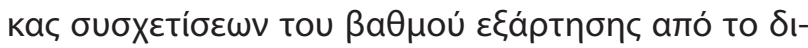

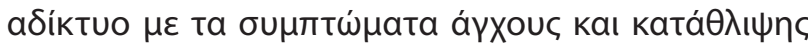
(ті́vakac 3).

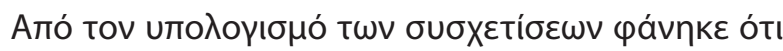

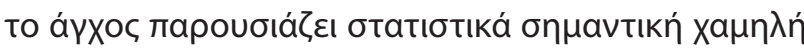

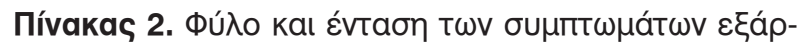

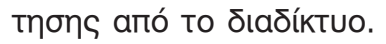

\begin{tabular}{|c|c|c|c|c|}
\hline & Фúภo & $\mathbf{n}$ & MO & TA \\
\hline \multirow{2}{*}{ 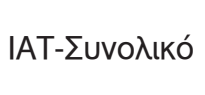 } & Гuvaíka & 19 & 51,1 & 14,7 \\
\hline & 'Avסрas & 156 & 46,5 & 18,7 \\
\hline
\end{tabular}

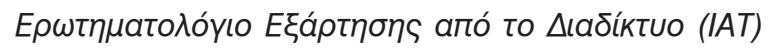

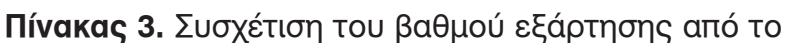

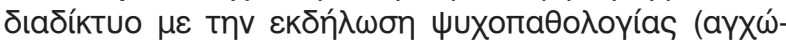

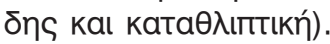

\begin{tabular}{|c|c|c|c|}
\hline & Ката́ $\theta \lambda ı \Psi \eta$ & 'AYXos & 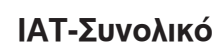 \\
\hline 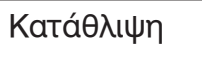 & & 0,07 & 0,12 \\
\hline 'Ayxos & & & $0,30^{*}$ \\
\hline 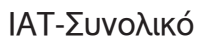 & & & \\
\hline
\end{tabular}

${ }^{*} \mathrm{p}<0,01$

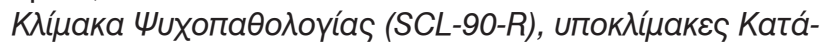
$\theta \lambda ı \psi \eta$ к каı Ayxous

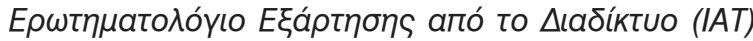

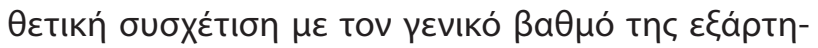

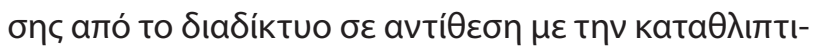

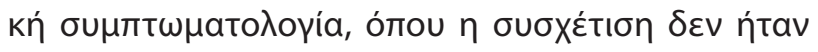

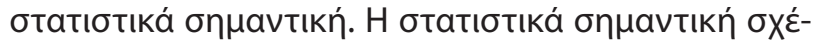

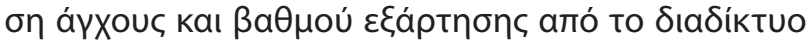

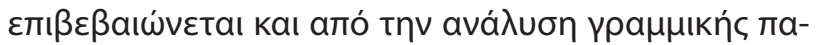

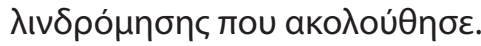

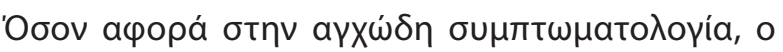

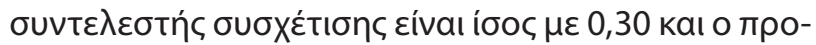

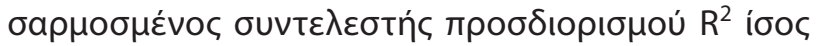

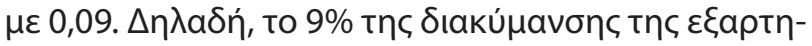

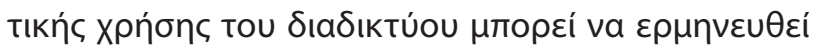

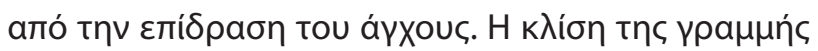

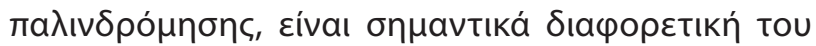

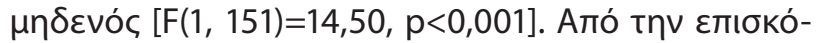

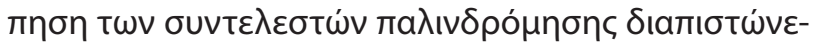
taı ótı тo ápXoৎ ( $\beta=0,30, t=3,81, p<0,01) \sigma \cup \mu \beta a ́ \lambda \lambda \varepsilon ı$

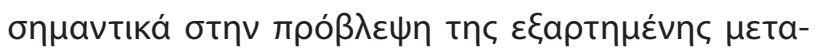

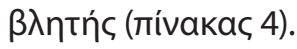

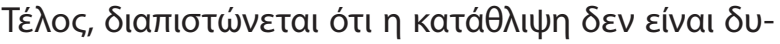

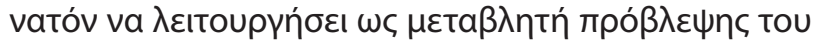

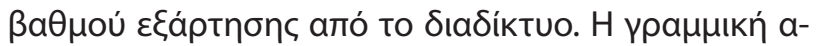

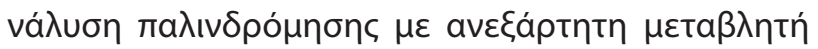

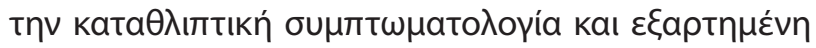

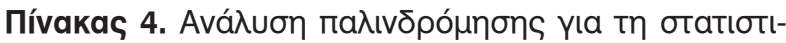

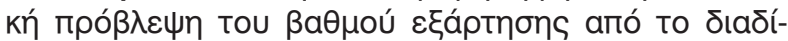

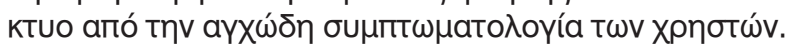

\begin{tabular}{|c|c|c|c|}
\hline $\begin{array}{l}\text { Проßגєптіко́ৎ } \\
\text { пара́үоvтая }\end{array}$ & B & SE B & $\beta$ \\
\hline 'AYxos & 6,70 & 1,76 & 0,30 * \\
\hline
\end{tabular}




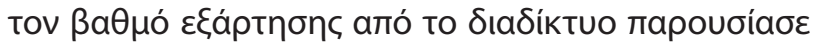

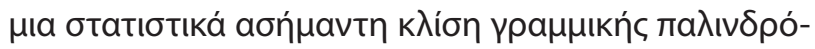

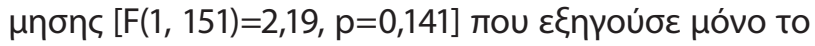

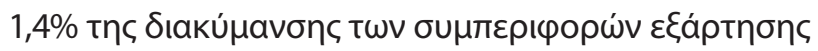

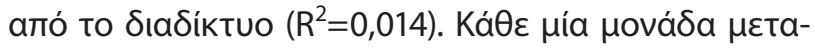

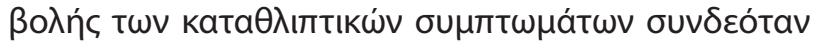

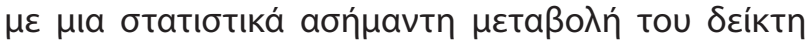

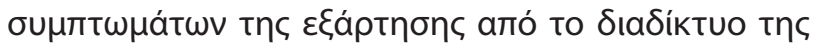

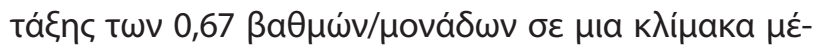

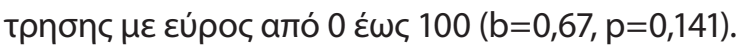

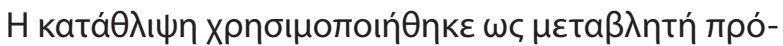

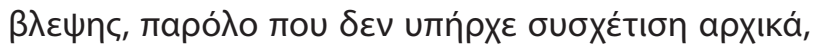

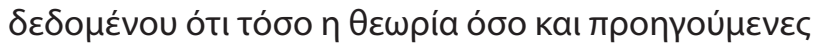

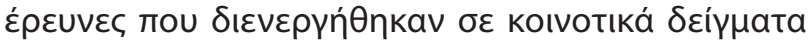

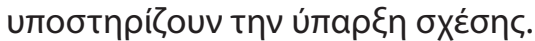

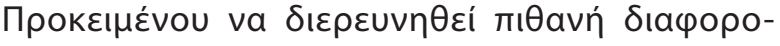

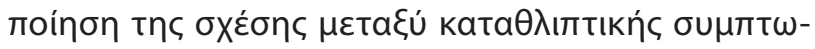

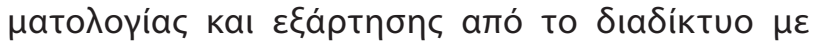

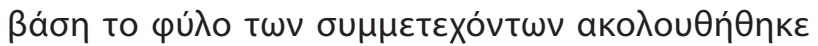

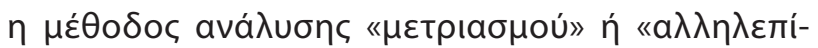

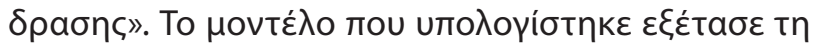

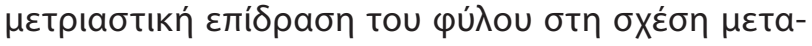

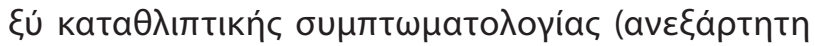

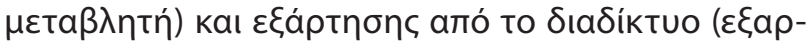

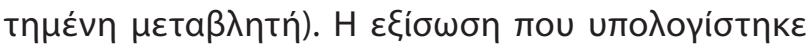

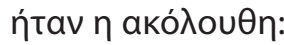

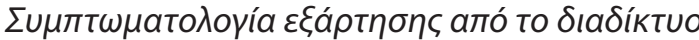

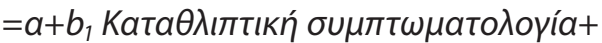

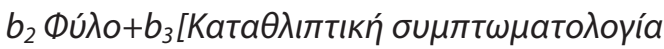

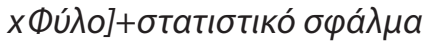

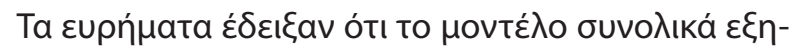

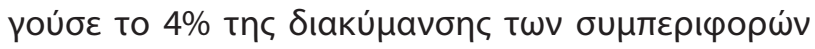

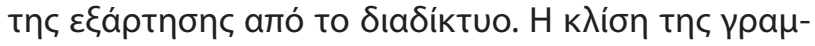

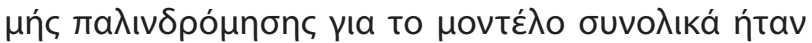

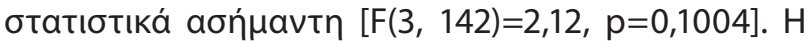

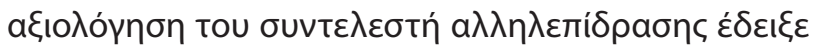

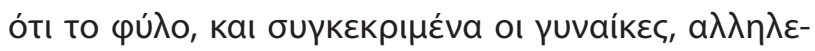

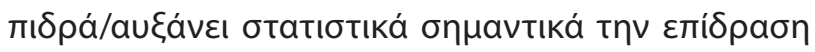

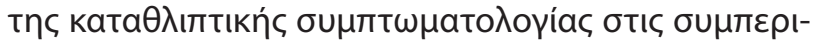

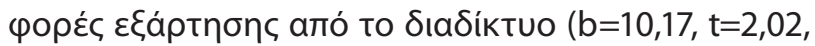

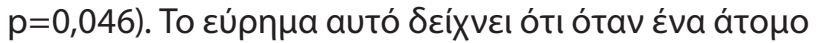

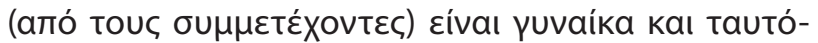

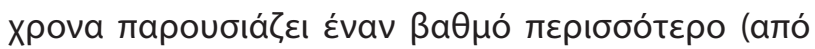

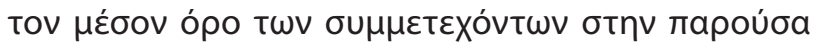

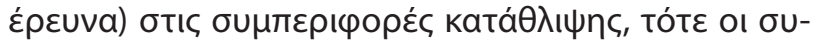

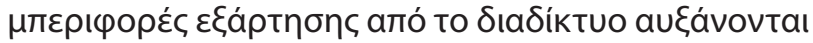

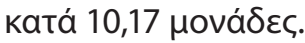

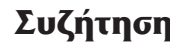

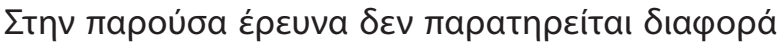

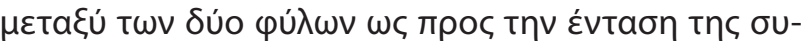

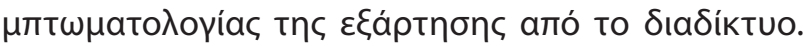

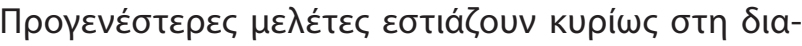

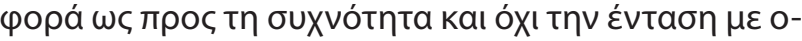

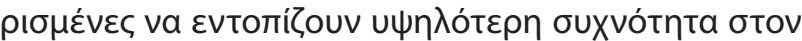

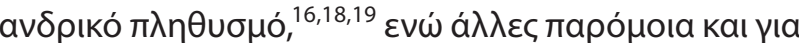

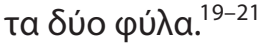

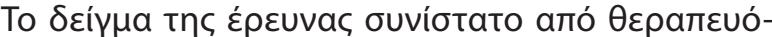

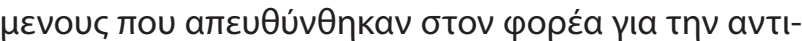

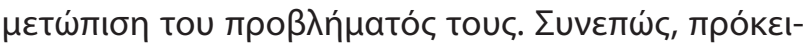

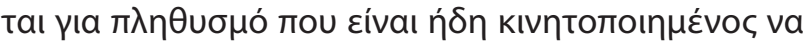

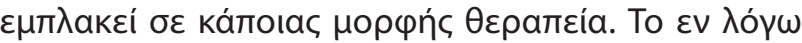

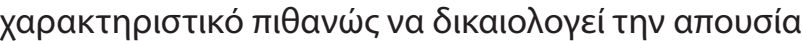

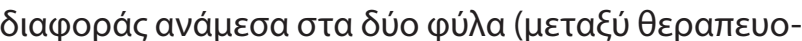

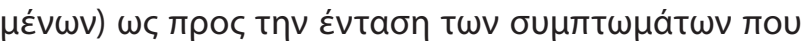

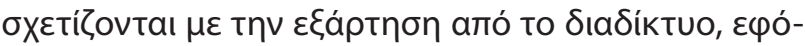

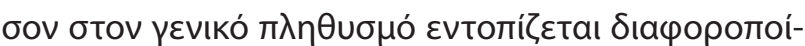

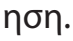

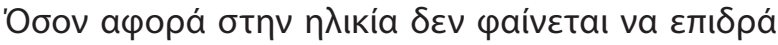

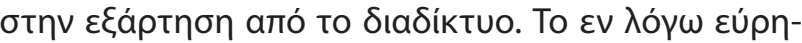

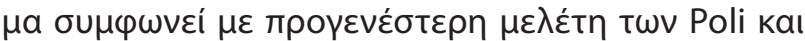

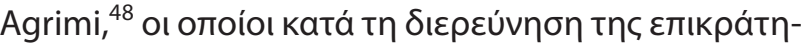

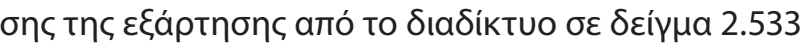

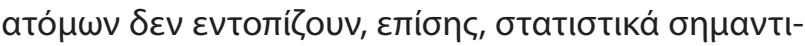

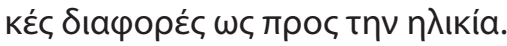

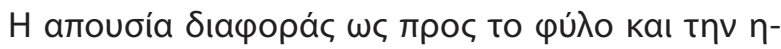

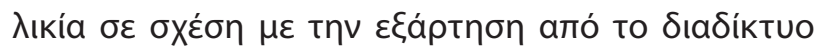

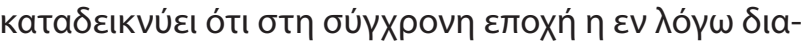

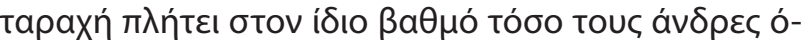

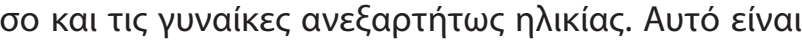

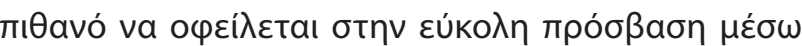

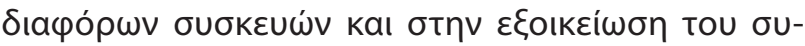

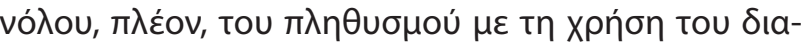

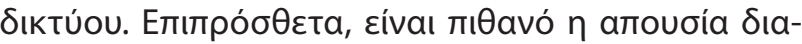

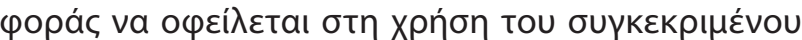

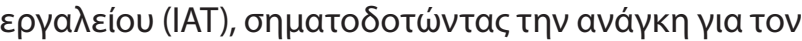

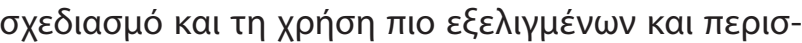

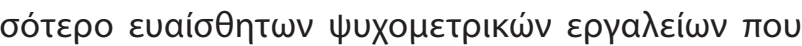

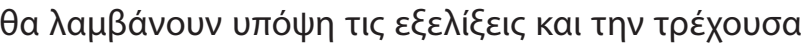
праүнатіко́тпта.

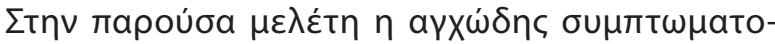

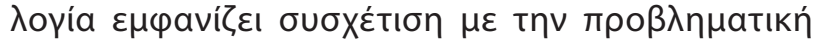

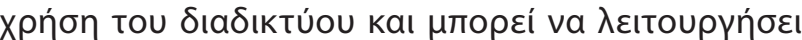

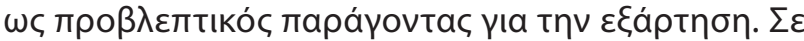




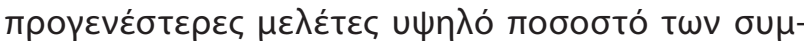

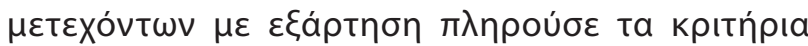

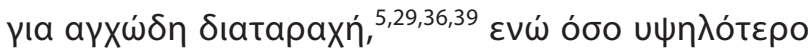

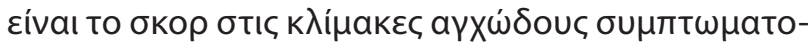

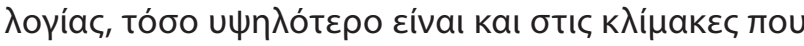

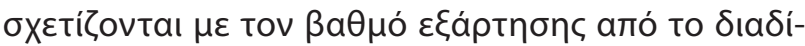
KTUO. ${ }^{36,39}$

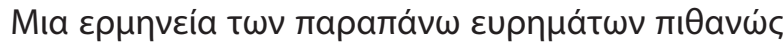

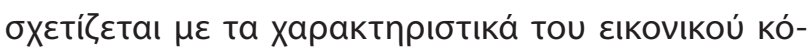

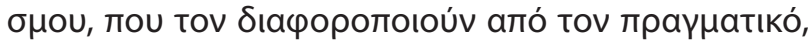

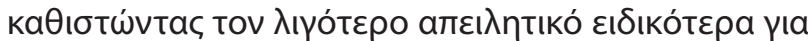

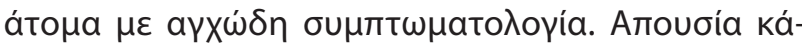

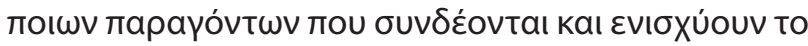

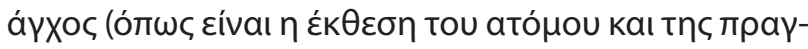

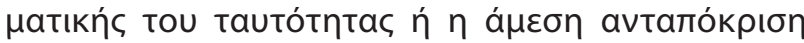

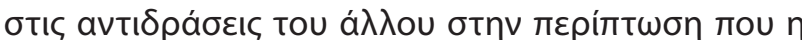

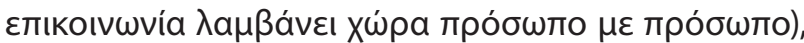

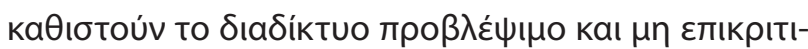

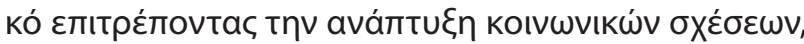

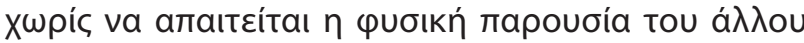

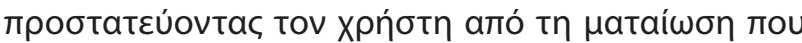

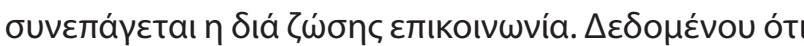

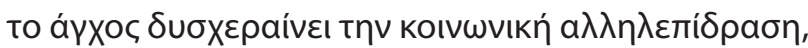

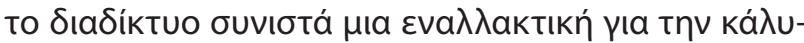

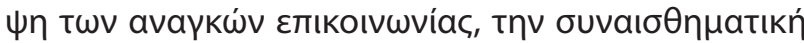

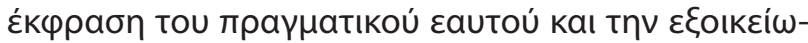

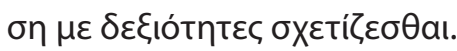

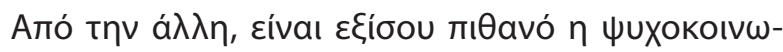

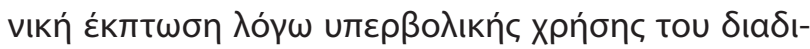

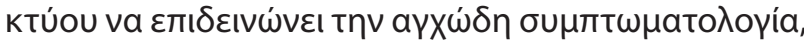

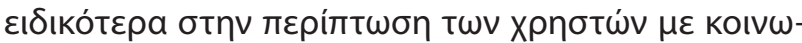

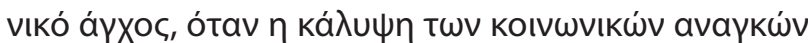

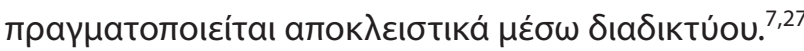

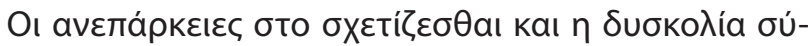

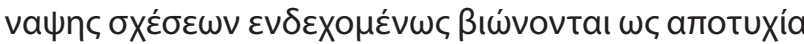

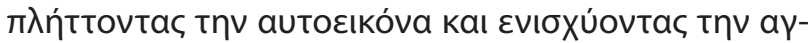

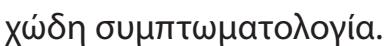

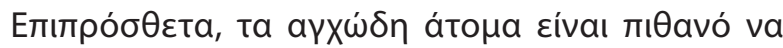

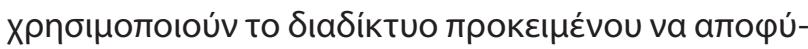

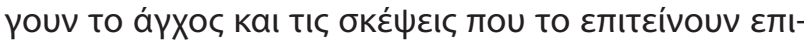

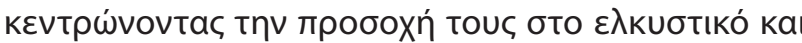

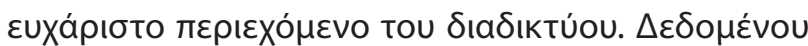

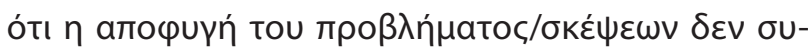

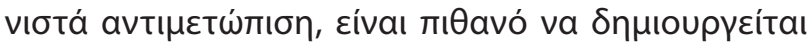

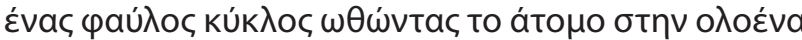

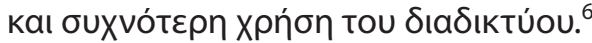

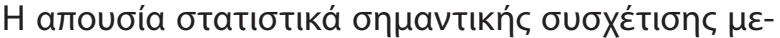

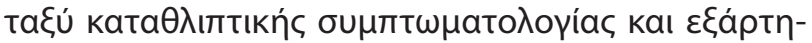

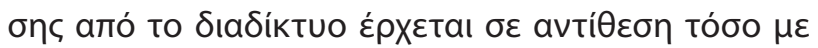

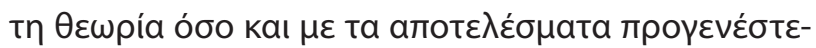

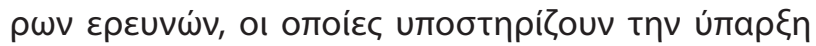

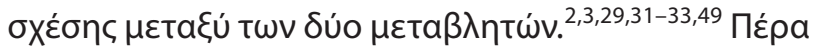

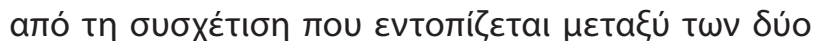

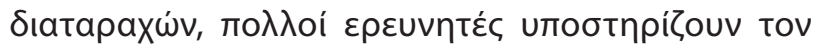

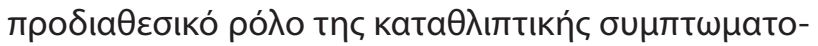

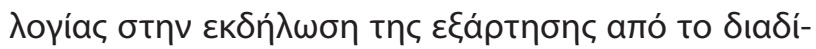

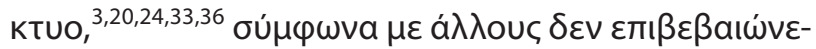

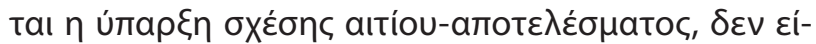

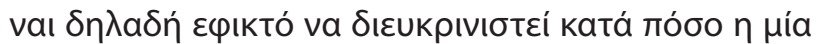

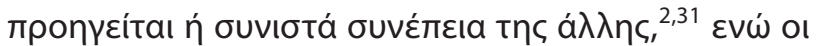

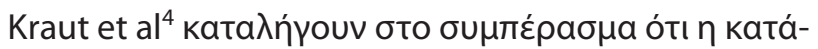

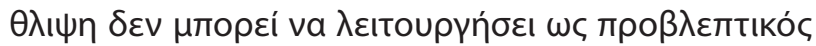

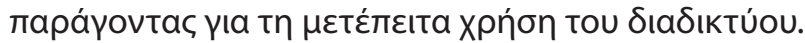

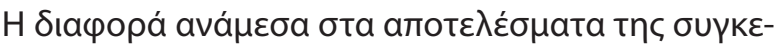

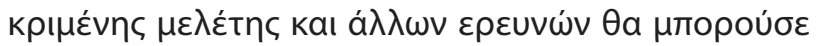

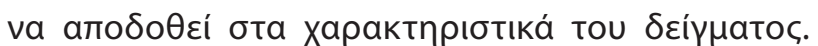

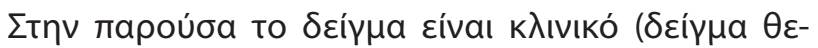

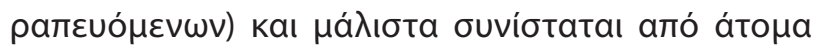

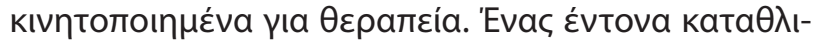

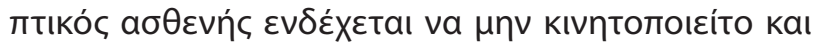

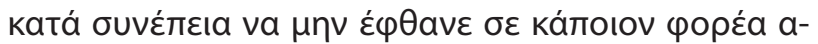

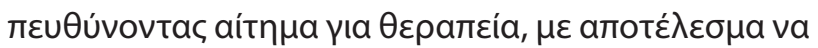

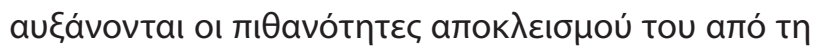

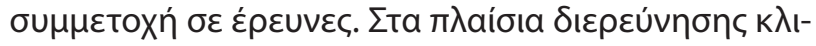

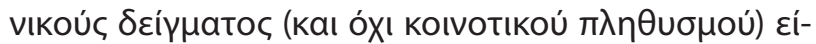

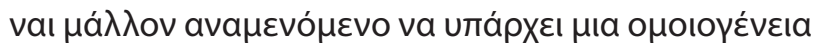

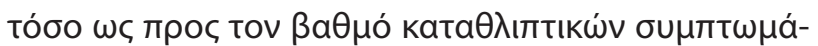

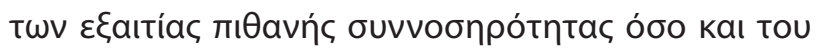

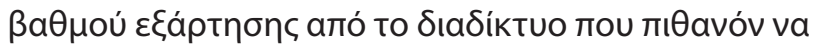

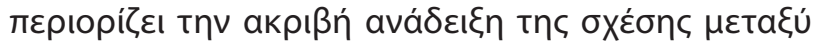

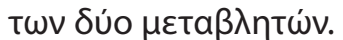

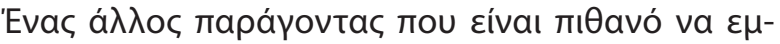

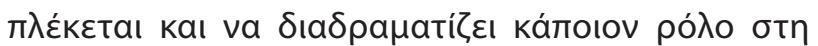

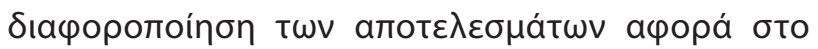

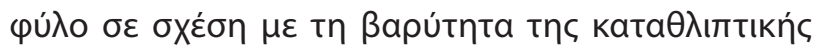

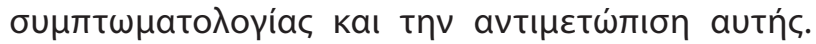

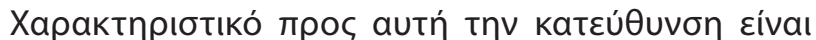

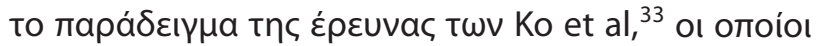

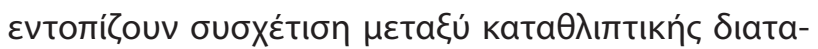

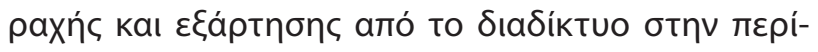

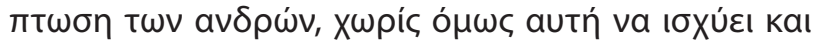

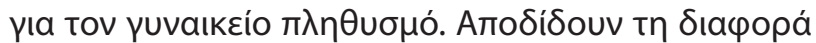

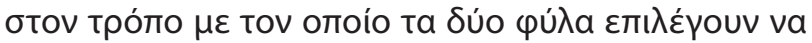




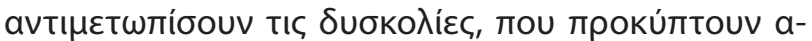

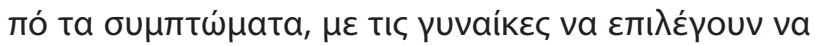

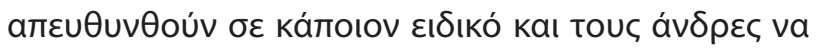

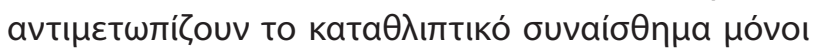

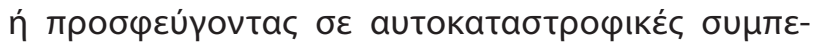

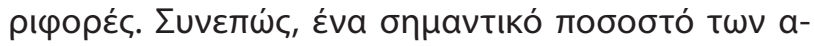

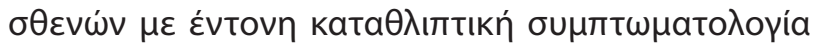

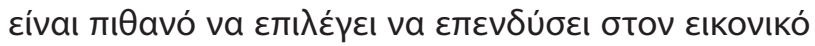

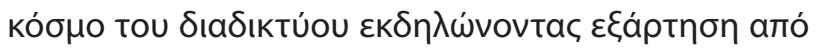

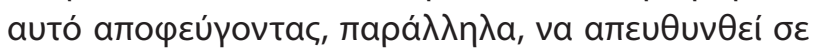

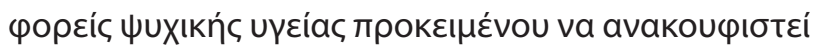
aтó to $\delta$

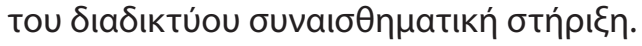

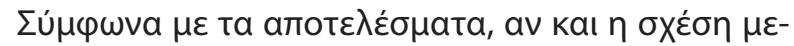

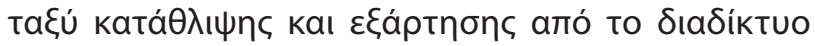

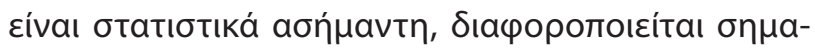

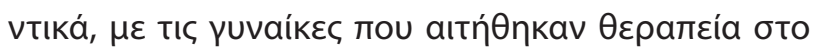

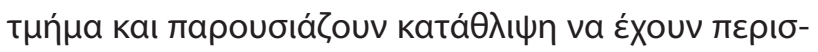

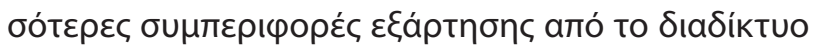

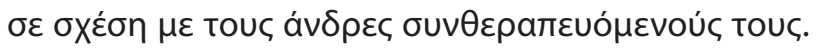

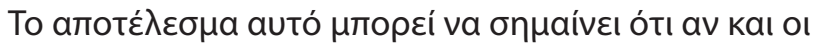

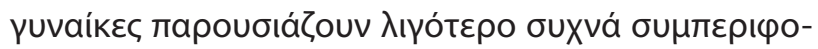

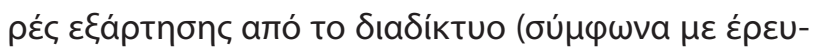

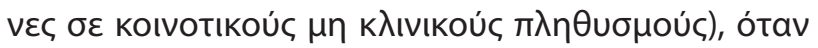

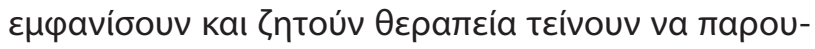

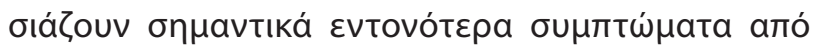

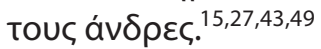

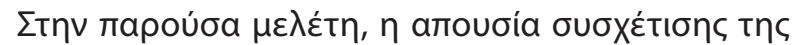
катаӨ

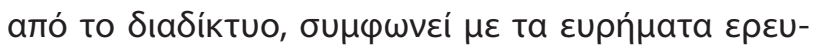

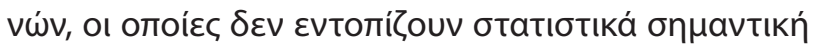

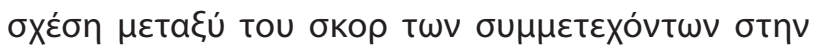

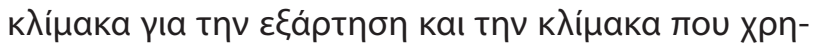

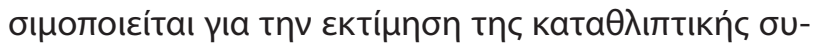

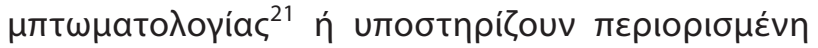

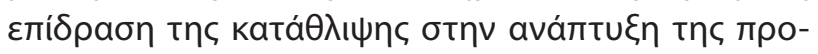

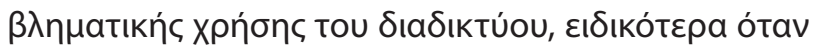

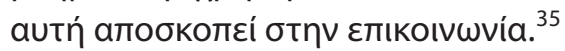

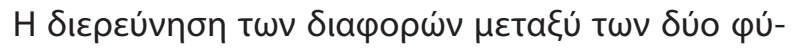

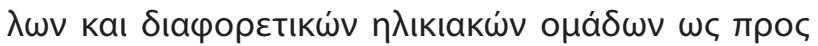

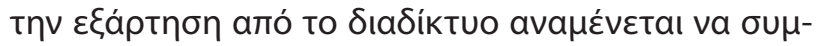

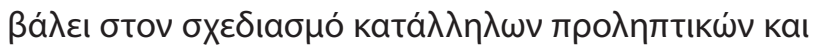

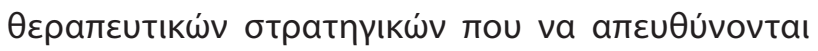

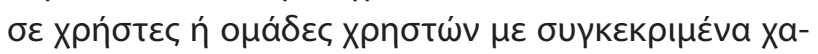

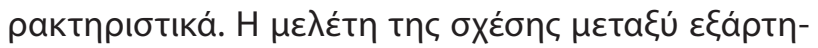

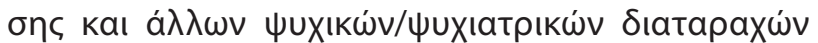

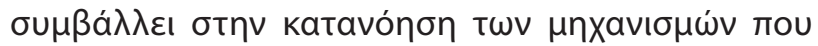

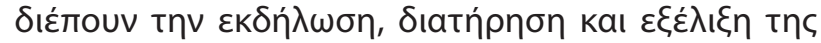

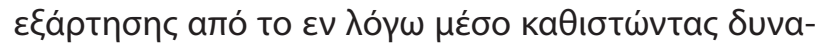

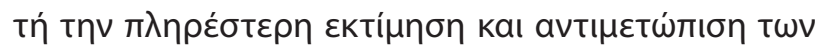

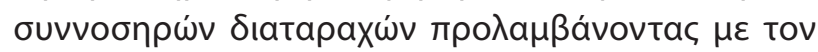

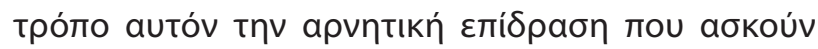

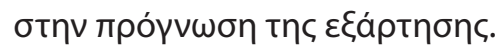

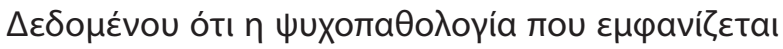

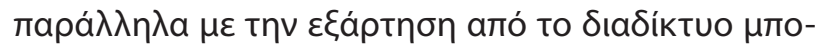

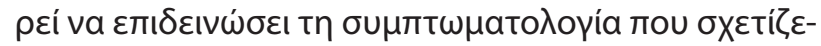

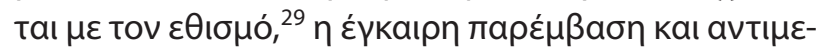

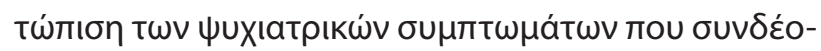

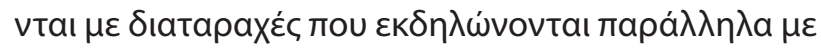

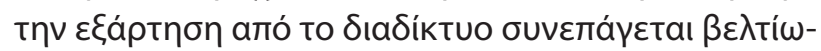

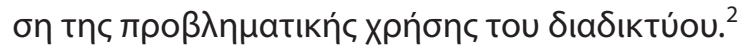

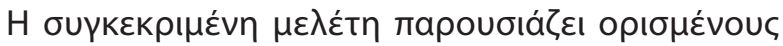

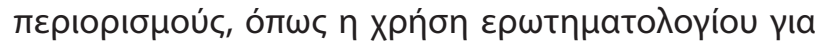

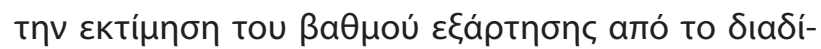

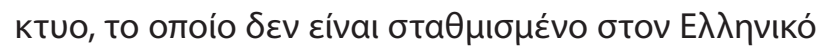

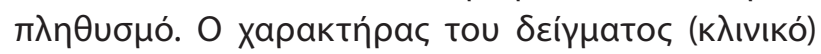

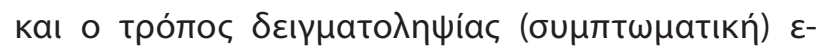

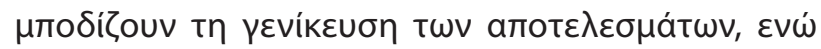

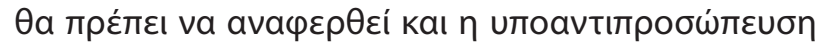

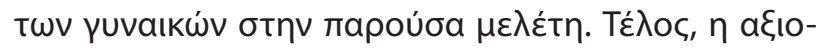

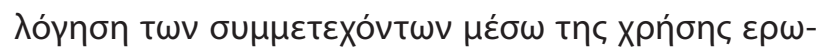

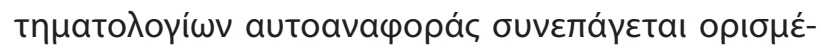

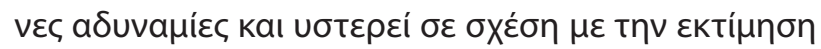

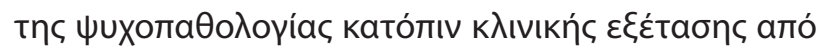

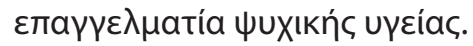

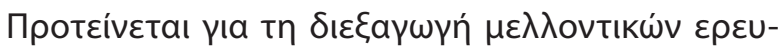

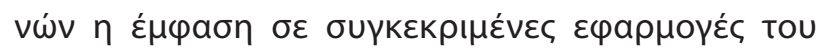

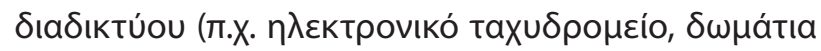

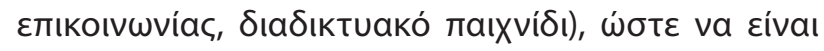

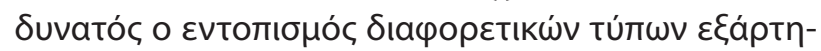

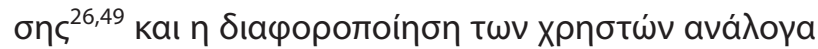

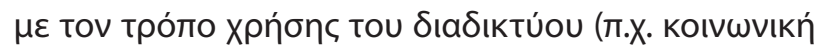

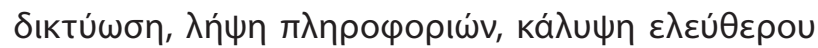

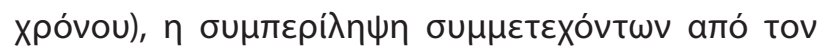

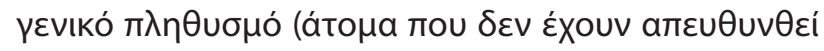

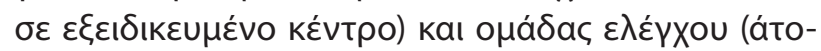

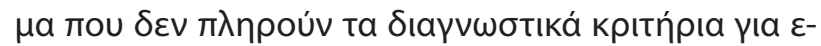

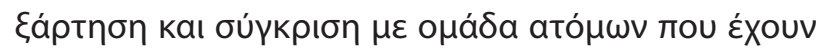

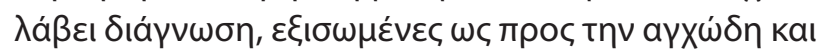

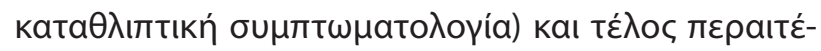

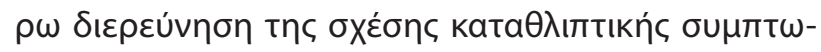

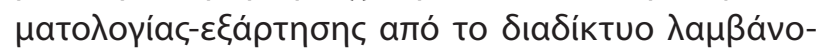

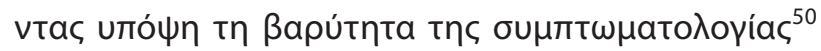

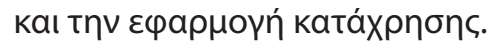




\title{
The relationship of internet addiction with anxiety and depressive symptomatology
}

\author{
E. Soulioti, V. Stavropoulos, S. Christidi, \\ Y. Papastefanou, P. Roussos \\ Department of 18 ANO Dependence Treatment Unit, Department for Problematic Use of Internet (Adults), \\ Psychiatric Hospital of Attica, Athens, Greece
}

Psychiatriki 2018, 29:160-171

\begin{abstract}
Internet stimulates the user's senses causing a variety of subjective experiences and sensations, even though it has no inherent addictive quality. These experiences could be positive, as the improvement of education, or adverse, as the development of internet addiction. There are many people who prefer to invest their time and energy in the virtual world of the internet. They choose to withdraw their emotional investments from face to face communication, while in some cases the internet connection signifies the user's disconnection from the real life, as the person is isolated from the surrounding and lives in a virtual environment. Under these conditions the excessive use of internet may lead to addiction. The purpose of the present study was to investigate the relationship between internet addiction and anxiety and depressive symptomatology of the user. Participants were 203 internet users aged between 17 and 58 years (Mean=26.03, SD=7.92) who approached the Department For Problematic Use Of Internet, Addiction Unit "18ANO" in Psychiatric Hospital Of Attica to receive specialized help for their pathological internet use. Internet Addiction Test (IAT) was used for the assessment of internet addiction and Symptom Checklist90-R (SCL-90-R) was administered for the evaluation of anxiety and depressive symptomatology. The analysis of the survey data showed that gender difference is not observed as to the intensity internet dependence. Younger users are more likely to develop addictive behaviour (in relation to internet use). At this point it should be noted that although positive, this association does not present to be statistically significant. Finally, regarding the relationship between psychopathology and internet addiction, anxiety symptomatology, which was moderately correlated with the overall score at IAT, was found to predict in regression analysis the internet addiction. There was no statistically significant association between internet addiction and depressive symptomatology, with women however, who presented with depressive symptoms to appear more vulnerable than men (who requested therapy from the department). Exploration of the effects of sex and age on internet addiction is expected contribute to the design of the appropriate preventive and therapeutic programs, whereas the study of the relationship between internet addiction and other psychiatric disorders would contribute the understanding of the mechanisms underpinning the development and onset of the addiction.
\end{abstract}

Key words: Internet addiction, psychopathology, anxiety symptoms, depressive symptoms.

\section{BıBntoypacpía}

1. Young KS. Internet addiction: A new clinical phenomenon and its consequences. Am Behav Scient 2004, 48:402-415, doi: $10.1177 / 0002764204270278$

2. Young KS, Rogers RC. The relationship between depression and internet addiction. Cyberpsycholo Behav 1998, 1:25-28, doi: 10.1089/cpb.1998.1.25

3. Young KS. Internet addiction: symptoms, evaluation and treatment. In: Vande-Creek L, Jackson TL (ed) Innovations in clinical practice: a source book. Vol.17 Professional Resource Press, Sarasota FL, 1999:19-31

4. Kraut R, Patterson M, Lundmark V, Kiesler S, Mukophadhyay T, Scherlis W. Internet paradox: a social technology that reduces social involvement and psychological well-being? Am Psychol 1998, 53:1017-1031, PMID: 9841579

5. Shapira NA, Goldsmith TD, Keck PE, Khosia UM, McElroy SL. Psychiatric features of individuals with problematic Internet use. J Affect Disord 2000, 57:267-272, PMID: 10708842 
6. Stavropoulos V, Gentile D, Motti-Stefanidi F. A multilevel Iongitudinal study of adolescent internet addiction: The role of obsessive-compulsive symptoms and classroom openness to experience. Eur J Dev Psychol 2016, 13: 99-114, doi: 10.1186/ s12991-017-0165-z

7. Stavropoulos V, Gomez R, Steen E, Beard C, Liew L, Griffiths MD. The longitudinal association between anxiety and Internet addiction in adolescence: The moderating effect of classroom extraversion. J Behav Addict 2017, 6:237-247, doi: 10.1556/2006.6.2017.026

8. Kuss DJ, Griffiths MD, Pontes HM. Chaos and confusion in DSM-5 diagnosis of Internet Gaming Disorder: Issues, concerns, and recommendations for clarity in the field. J Behav Addict 2017, 6:103-109, doi: 10.1556/2006.5.2016.062

9. Griffiths MD, Kuss DJ, Billieux J, Pontes HM. The evolution of Internet addiction: A global perspective. Addict Behav 2016, 53: 193-195, doi: 10.1016/j.addbeh.2015.11.001

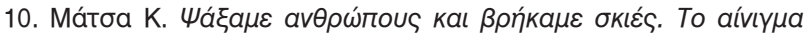

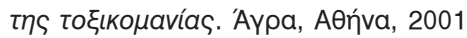

11. Pontes HM, Griffiths MD. Internet addiction disorder and internet gaming disorder are not the same. J Addict Res Ther 2014, 5, doi: 10.4172/2155-6105.1000e124

12. American Psychiatric Association. Diagnostic and Statistical Manual of Mental Disorders. 5th ed. American Psychiatric Publishing, Arlington VA, 2013

13. Christakis DA. Internet addiction: a 21st century epidemic? BMC Medicine 2010, 8:1-3, doi: 10.1186/1741-7015-8-61

14. Ko CH, Yen JY, Yen CF, Chen CS, Che CC. The association between Internet addiction and psychiatric disorder: A review of the literature. Eur Psychiatry 2012, 27:1-8, doi: 10.1016/j. eurpsy.2010.04.011

15. Siomos D, Dafouli E, Braimiotis D, Mouzas O, Angelopoulos N. Internet addiction among Greek adolescent students. Cyberpsych Behav 2008, 11:653-657, Available from: http:// www.academia.edu/4032228/Internet_addiction_as_an_important_predictor_in_early_detection_of_adolescent_drug_use_ experience-implications_for_research_and_practice

16. Frangos CC, Frangos CC, Kiohos AP. Internet addiction among Greek university students: Demographic Associations with the Phenomenon, using the Greek version of Young's Internet Addiction Scale. Int J Econ Sc App Res 2010, 3:1-35, Available from: http://discovery.ucl.ac.uk/id/eprint/20369

17. Tsitsika A, Janikian M, Schoenmakers TM, Tzavela EC, Olafsson $\mathrm{K}$, Wojcik $\mathrm{S}$ et al. Internet addictive behaviour in adolescence: A cross-sectional study in seven European countries. Cyberpsychol Behav Soc Netw 2014, 17:528-53, doi: 10.1089/ cyber.2013.0382

18. Morahan-Martin J. Caught in the web: Research and criticism of the internet abuse with application to college students. In: Wolfe CR (ed) Learning and teaching on the World Wide Web. Academic Press, United States of America, 2001:191-220, doi: 10.1016/B978-012761891-3/50013-8

19. Yang SC, Tung CJ. Comparison of Internet addicts and nonaddicts in Taiwanese high school. Comput Hum Behav 2007, 23:79-96, Available from: https://pdfs.semanticscholar.org/735 2/925e375549e4a001990ff93af72b7805f149.pdf
20. Andreou E, Svoli H. The association between Internet user characteristics and dimensions of Internet addiction among Greek adolescents. Int J Ment Health Addiction 2013, 11:139-148, doi: 10.1007/s11469-012-9404-3

21. Kim K, Ryu E, Chon MY, Yeun EJ, Choi SY, Seo JS. Internet addiction in Korean adolescents and its relation to depression and suicidal ideation: A questionnaire survey. Int $J$ Nurs Stud 2006, 42:185-192

22. Koukia E, Mangoulia P, Alexiou E. Internet addiction and psychopathological symptoms in Greek university students. J Addict Behav Ther Rahabil 2014, 3:1-5, doi: 10.4172/23249005.1000125

23. Canan F, Ataoglu A, Ozcetin A, Icmeli C. The association between internet addiction and dissociation among Turkish college students. Compr Psychiatry 2012, 53:422-426, doi: 10.1016/ j.comppsych.2011.08.006

24. Evren C, Dalbudak E, Evren B, Demirci AC. High risk of Internet addiction and its relationship with lifetime substance use, psychological an behavioral problems among 10th grade adolescents. Psychiat Danub 2014, 26:330-339, Available from: file:///C:/Users/HPS-Journal/Downloads/Dnb_26_4_330_9 Evr\%20(2).pdf

25. Yates TM, Gregor MA, Haviland MG. Child maltreatment, alexithymia and problematic Internet use in young adulthood. Cyberpsych Beh Soc N 2012, 15:219-225, doi: 10.1089/cyber. 2011.0427

26. Widyanto L, Griffiths MD. Internet addiction: a critical review. Int J Ment Health Ad 2006, 4:31-51, Available from: http://www. academia.edu/429533/Widyanto_L._and_Griffiths_M.D._2006_. Internet_addiction_A_critical_review._International_Journal_of_ Mental_Health_and_Addiction_4_31-51

27. Anderson EL, Steen E, Stavropoulos V. Internet use and problematic internet use: A systematic review of longitudinal research trends in adolescence and emergent adulthood. Intern J Adolesc Youth 2016, 1-25, doi: 10.1080/02673843.2016.1227716

28. Block JJ. Issues for DSM-V: Internet addiction. Am J Psychiatry 2008, 165:306-307, doi: 10.1176/appi.ajp.2007.07101556

29. Floros G, Siomos K, Stogiannidou A, Giouzepas I, Garyfallos G. Comorbidity of psychiatric disorders with Internet addiction in a clinical sample: The effect of personality, defense style and psychopathology. Addict Behav 2014, 39:1839-1845, doi: 10.1016/j.addbeh.2014.07.031

30. Bernardi S, Pallanti S. Internet addiction: a descriptive clinical study focusing on comorbidities and dissociative symptoms, Compr Psychiatry 2009, 50:510-516, doi: 10.1016/j. comppsych.2008.11.011

31. Lee YS, Han DH, Yang KC, Daniels MA, Na C, Kee BS Depression like characteristics of 5HTTLPR polymorphism and temperament in excessive internet users. J Affect Disord 2008, 109:165-169, doi: 10.1016/j.jad.2007.10.020

32. Morrison $\mathrm{CM}$, Gore $\mathrm{H}$. The relationship between excessive internet use and depression: A questionnaire-based study of 1319 young people and adults. Psychopathology 2010, 43:121-126, doi: 10.1159/00027700

33. Ko CH, Yen JY, Chen CS, Chen CC, Yen CF. Psychiatric comorbidity of internet addiction in college students: an interview study. CNS Spectr 2008, 13:147-153, PMID: 18227746 
34. Whang LS, Lee S, Chang G. Internet over users' psychological profiles: a behavior sampling analysis on internet addiction. Cyberpsychol Behav 2003, 6:143-150, doi: 10.1089/109493103321640338

35. Caplan SE. Preference for online social interaction: a theory of problematic internet use and psychosocial well-being. Comm Res 2003, 30:625-648, Available from: https://soic.iupui.edu/ files/Thesis_content_example2.pdf

36. Akin A, Iskender M. Internet addiction and depression, anxiety and stress. IOJES 2011, 3:138-148, Available from: http://citeseerx. ist.psu.edu/viewdoc/download?doi=10.1.1.658.6294\&rep=rep1\&t ype $=$ pdf

37. McKenna KYA, Bargh JA. Plan 9 from cyberspace: The implications of the Internet for personality and social psychology. PSPR 2000, 4:57-75, doi: 10.1207/S15327957 PSPR0401_6

38. Kraut R, Kiesler S, Boneva B, Cummings J, Helgeson V, Crawford A. Internet paradox revisited. J Soc Issues 2002, 58: 49-74, doi: 10.1111/1540-4560.00248

39. Hardie E, Tee MY. Excessive Internet Use: The role of personality, Ioneliness and social support networks in Internet addiction. AJETS 2007, 5:34-47, Available from: http://ictaugustine. pbworks. com/f/Excessive+Internet+Use.pdf

40. Montag C, Jurkiewicz M, Reuter M. Low self-directedness is a better predictor for problematic internet use than high neuroticism. Comput Human Behav 2010, 26:1531-1535, doi: 10.1016/j. chb.2010.05.021

41. Siomos K, Floros G, Fisoun V, Dafouli E, Farkonas N, Sergentani $\mathrm{E}$ et al. Evolution of internet addiction in Greek adolescent students over a two-year period: the impact of parental bonding. Eur Child Adolesc Psychiatry 2012, 21:211-219, Available from: https://www.researchgate.net/publication/221811535_Evolution_ of_Internet_addiction_in_Greek_adolescent_students_over_a_ two-year_period_The_impact_of_parental_bonding

42. Leung L, Lee PSN. Impact of internet literacy, internet addiction symptoms, and internet activities on academic performance. Soc Sci Comput Rev 2012, 30:403-418, doi: 10.1177/ 0894439311435217

43. Stavropoulos V, Alexandraki K, Motti-Stefanidi F. Recognizing internet addiction: Prevalence and relationship to academic achievement in adolescents enrolled in urban and rural Greek high schools. J Adolesc 2013, 36:565-576

44. Derogatis LR. Symptom Checklist-90-R: Administration, scoring and procedures manual. 3rd ed. National Computer Systems Inc, Minneapolis, 1994

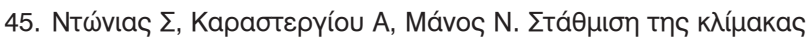

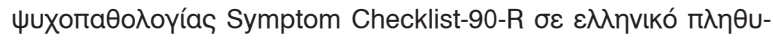

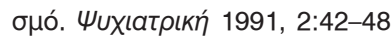

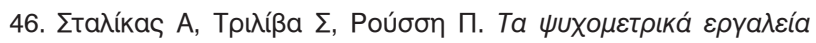

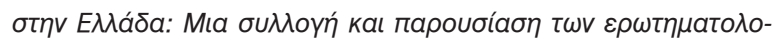

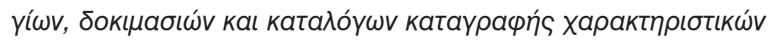

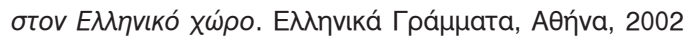

47. Berkovits I, Hancock GR, Nevitt J. Bootstrap resampling approaches for repeated measure designs: Relative robustness to sphericity and normality violations. Educ Psychol Meas 2000, 60:877-892, doi: 10.1177/00131640021970961

48. Poli R, Agrimi E. Internet addiction disorder: Prevalence in an Italian student population. Nord J Psychiatry 2012, 66: 55-59, doi: 10.3109/08039488.2011.605169

49. Kuss DJ, Griffiths MD, Binder JF. Internet addiction in students: Prevalence and risk factors. Comput Human Behav 2013, 29:959-966, doi: 10.1016/j.chb.2012.12.024

50. Bessière K, Kiesler S, Kraut R, Boneva BS. Effects of Internet use and social resources on changes in depression. Inform Commun \& Soc 2008, 11:47-70, doi: 10.1080/13691180701858851

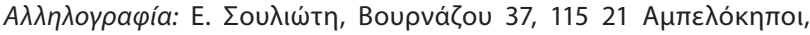

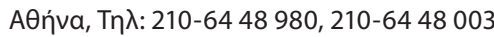

e-mail: net18ano@gmail.com,www.18ano.gr 


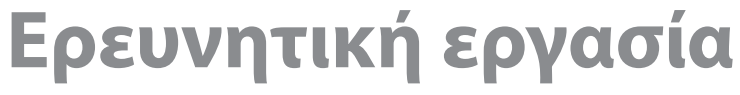 Research article
}

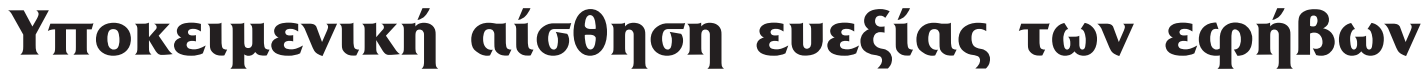 kal o póños tins olkoyéveias}

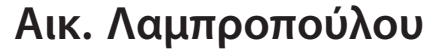

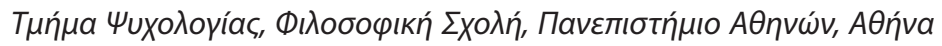

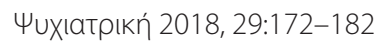

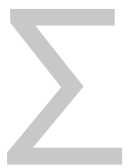

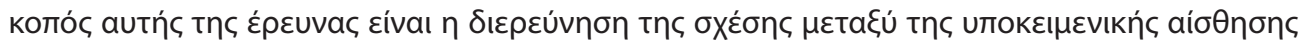

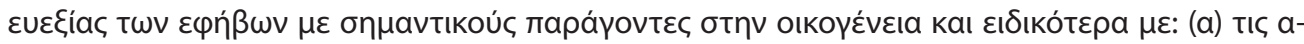

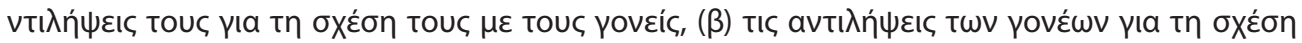

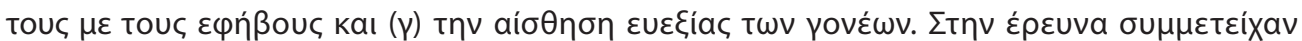

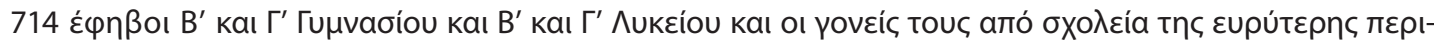

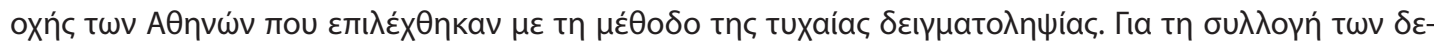

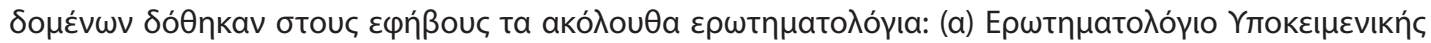

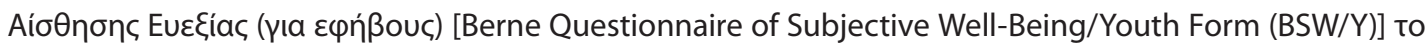

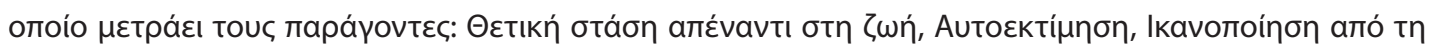

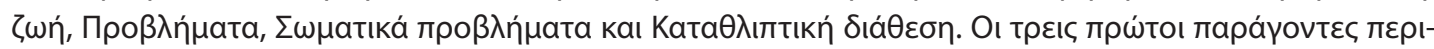

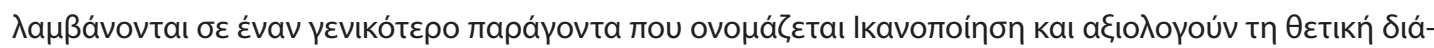

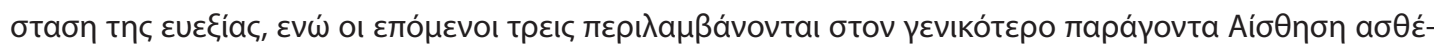

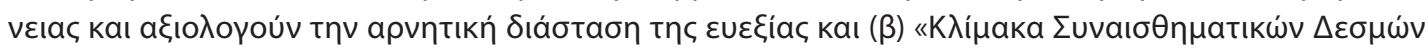

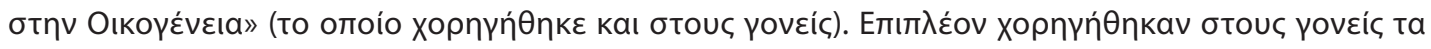

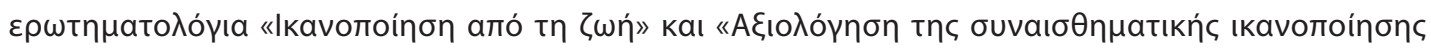

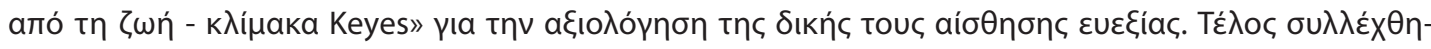

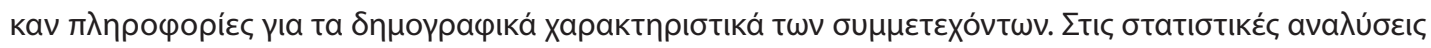

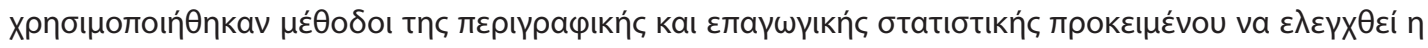

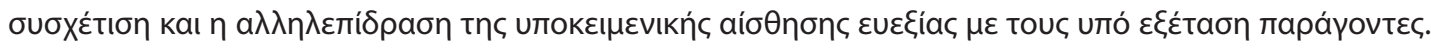

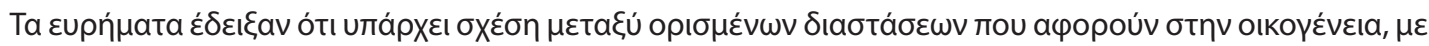

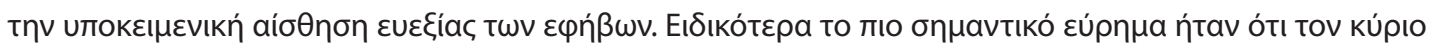

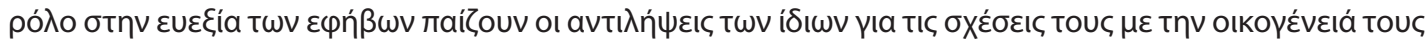

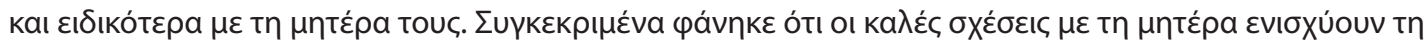

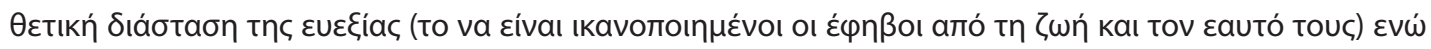




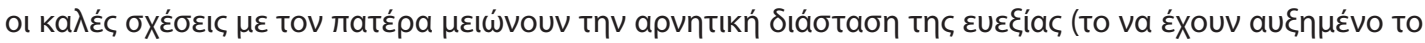

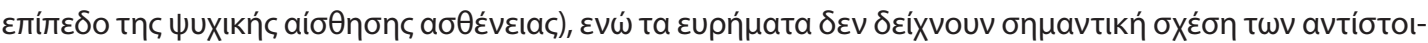

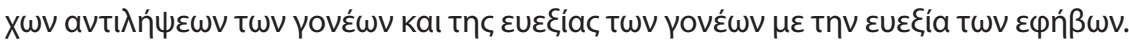

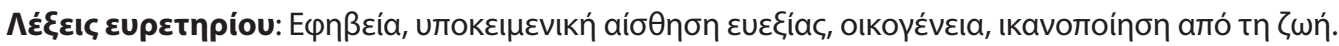

\section{Eıбaywyń}

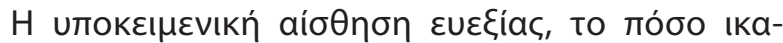

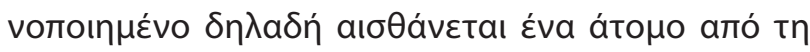

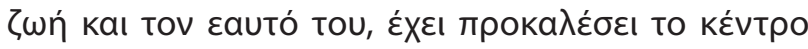

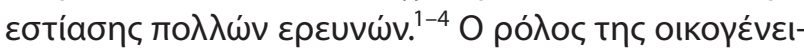

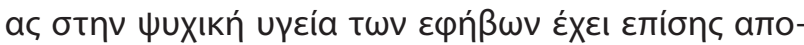

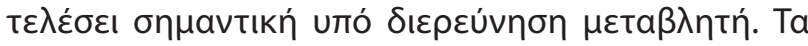

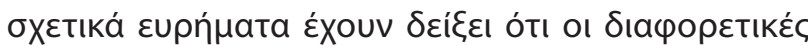

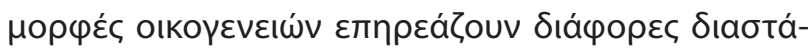

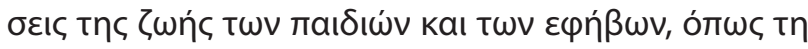

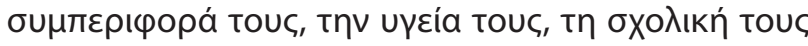

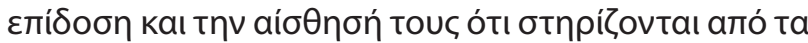

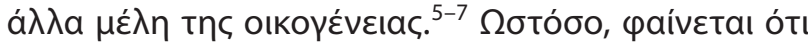

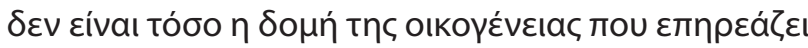

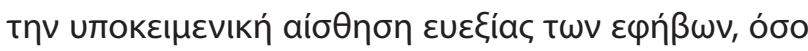

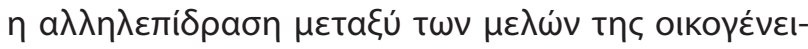

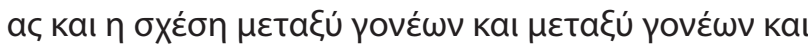
паı $\_\omega ́ v .8$

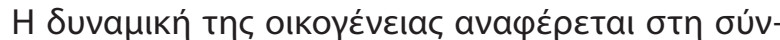

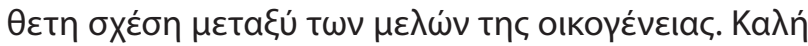

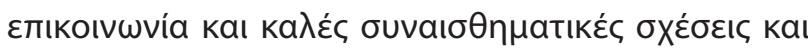

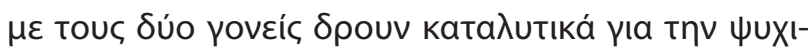

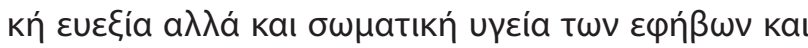

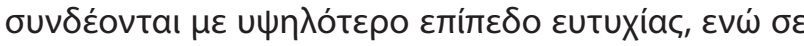

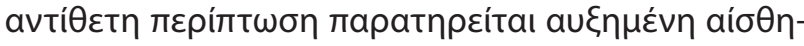

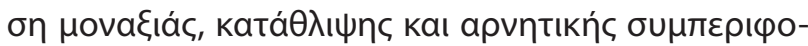
pác. 9,10

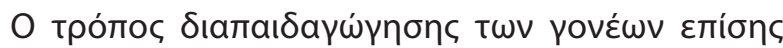

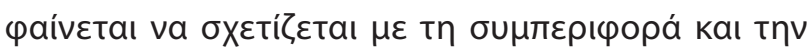

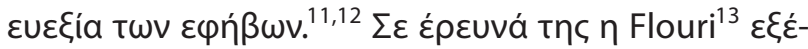

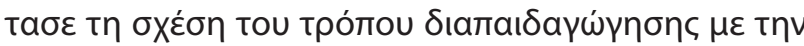

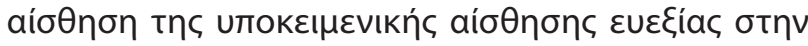

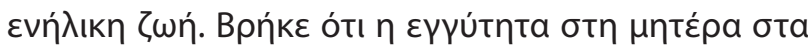

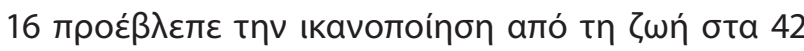

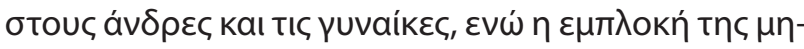

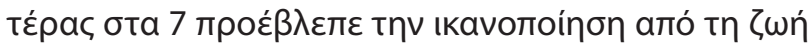

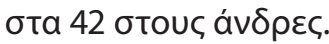

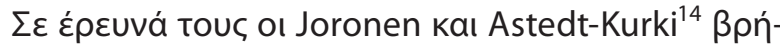

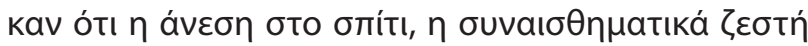

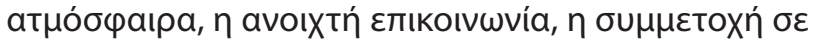

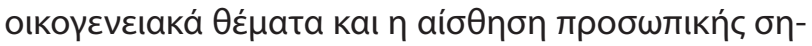

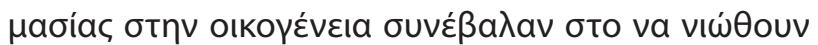

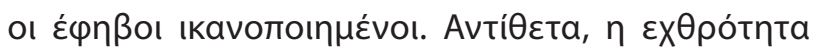

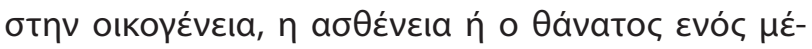

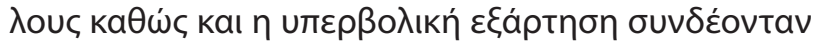

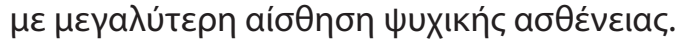

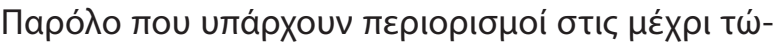

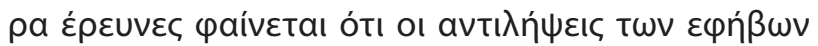

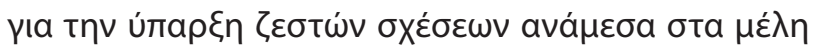

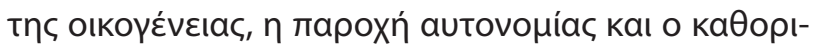

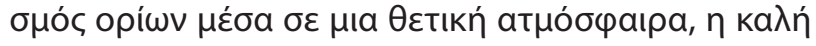

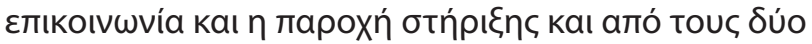

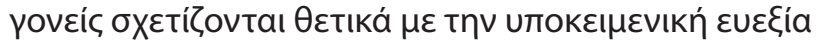
$\tau \omega v \varepsilon \varphi n ́ \beta \omega v$.

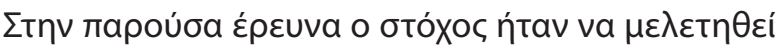

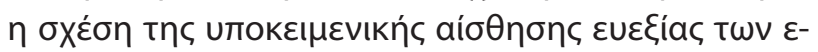

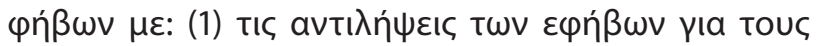

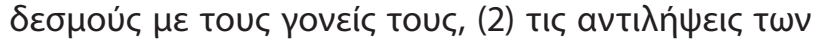

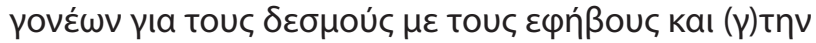

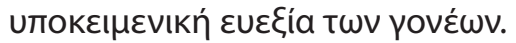

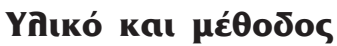

\section{$\Delta \varepsilon i ́ y \mu a$}

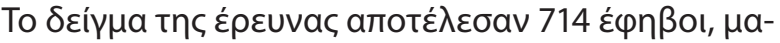

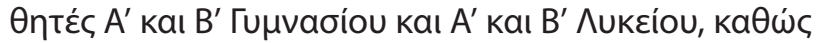

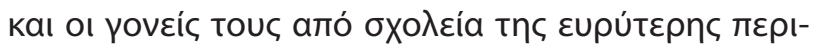

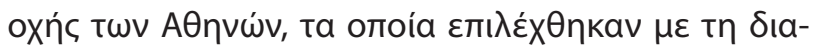

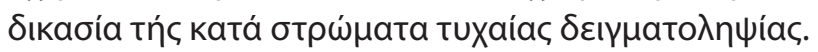

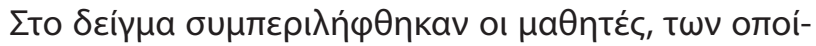

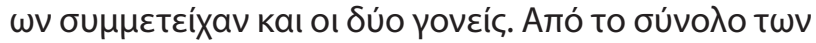

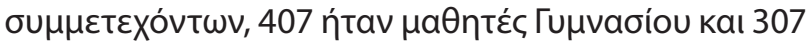

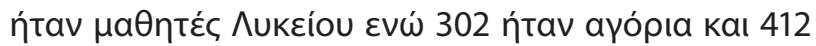
ńtav kopítoıa.

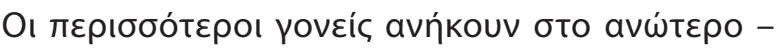

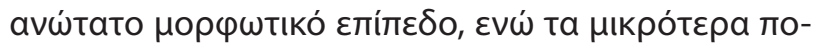

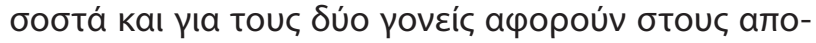

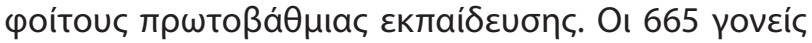

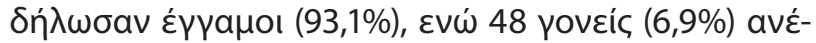




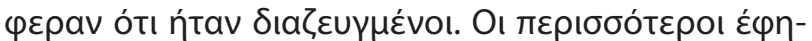

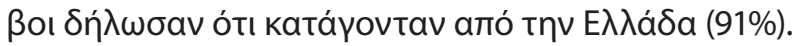

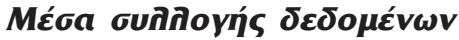

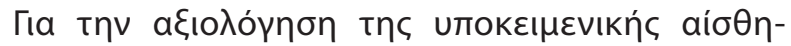

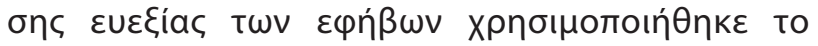

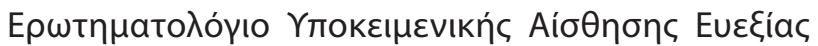

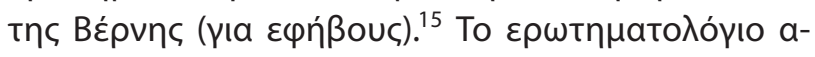

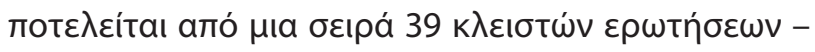

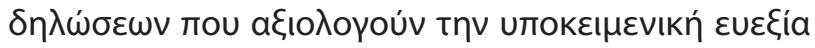

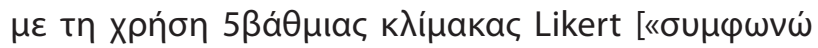

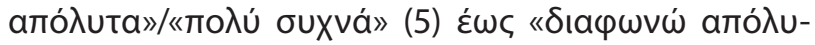

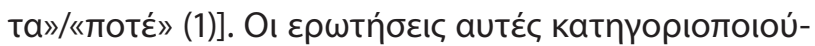

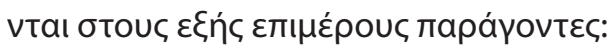

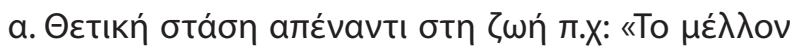

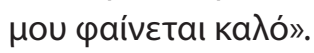

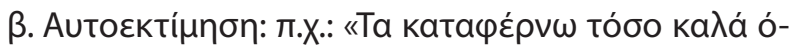

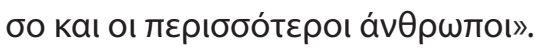

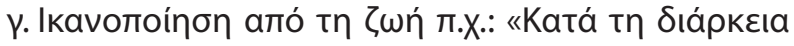

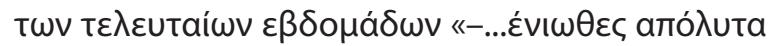

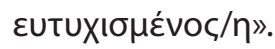

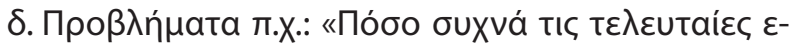

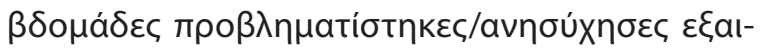

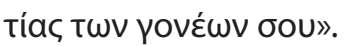

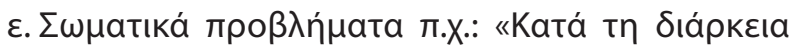

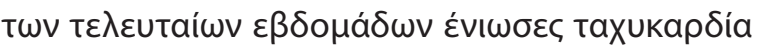

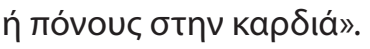

бт. КатаӨ пота пıа».

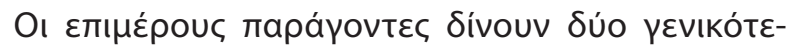

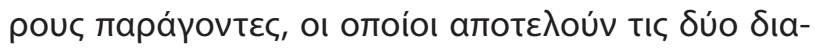

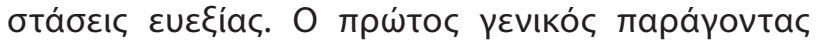

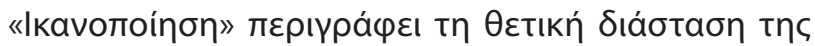

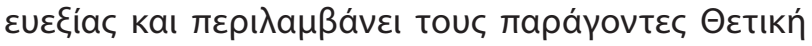

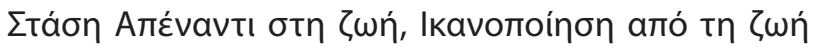

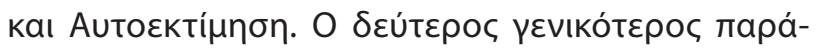

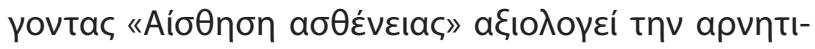

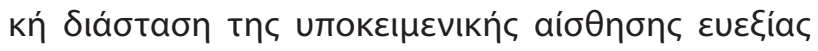

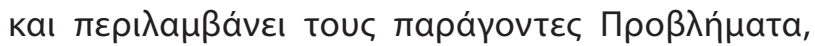

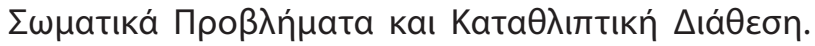

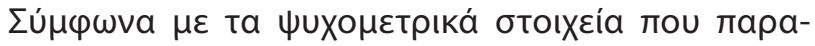

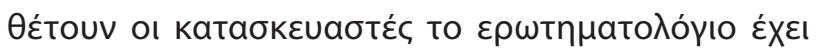

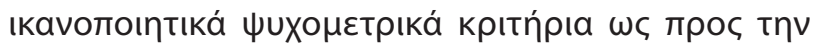

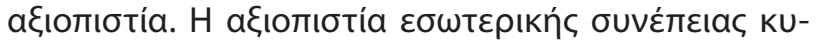

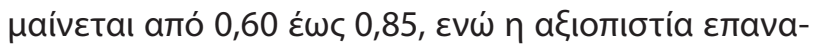

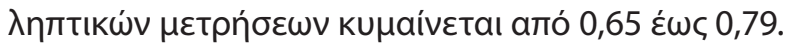

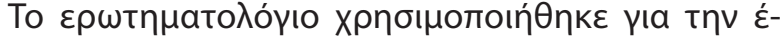

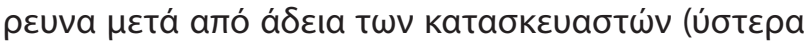

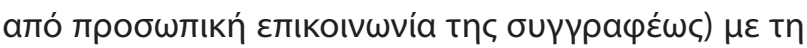

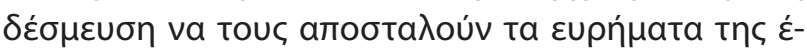

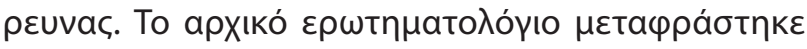

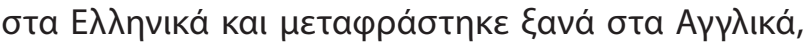

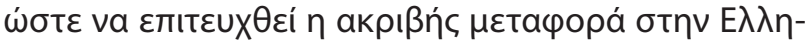

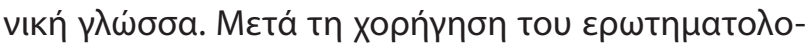

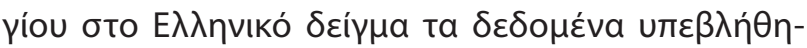

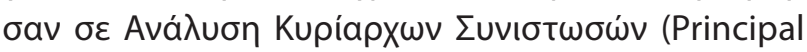

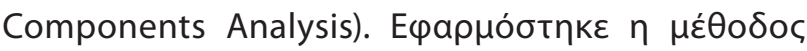

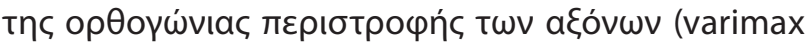

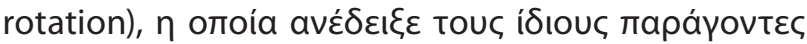

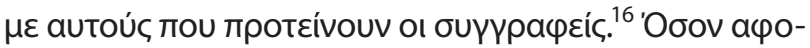

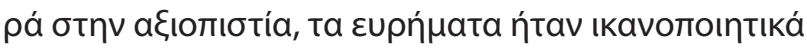

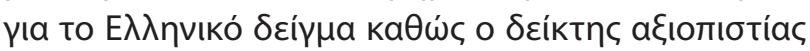

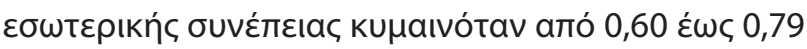

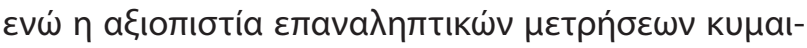

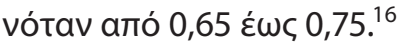

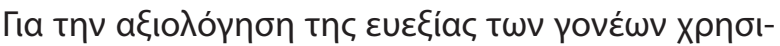
нотопиंӨпкаv:

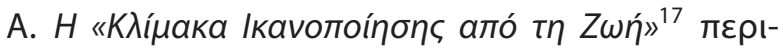

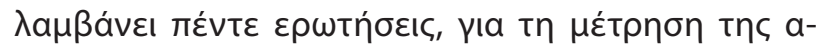

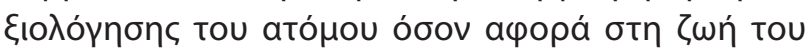

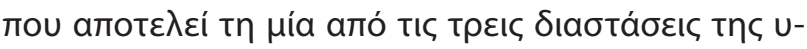

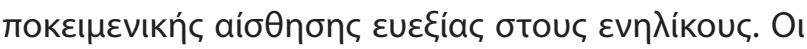

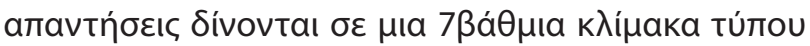

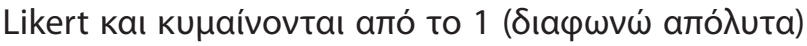

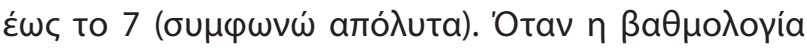

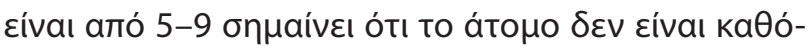

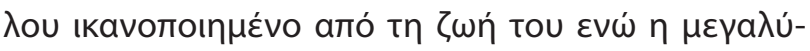

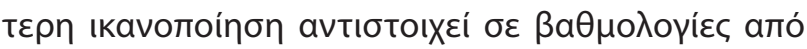

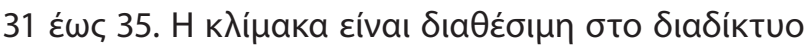

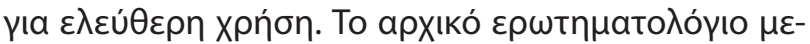

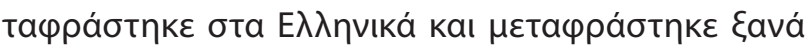

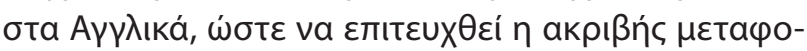

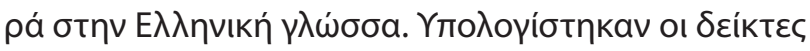

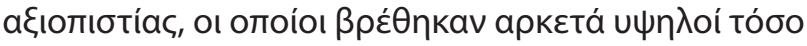

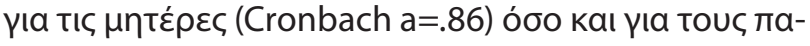

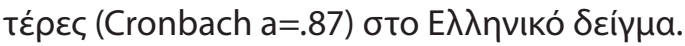

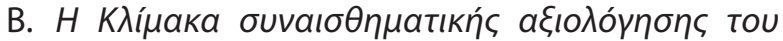

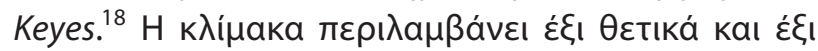

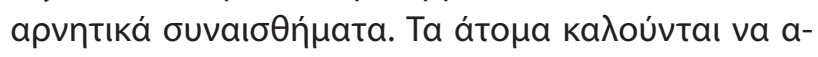

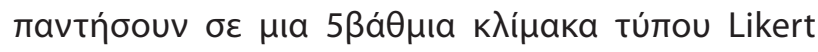

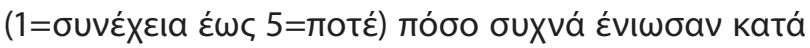

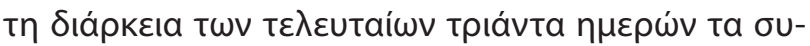

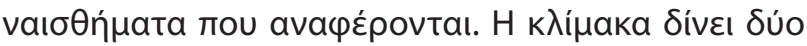




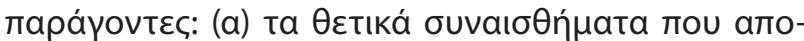

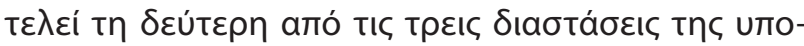

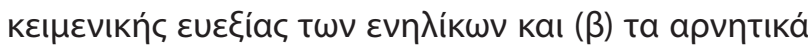

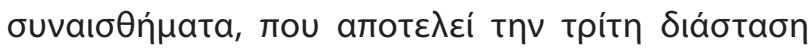

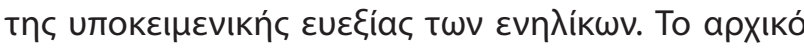

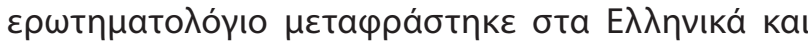

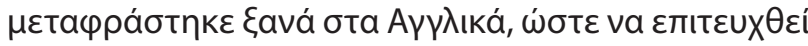

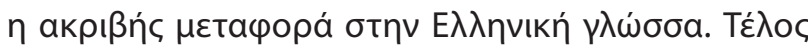

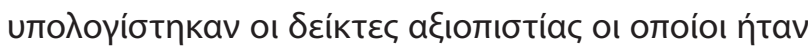

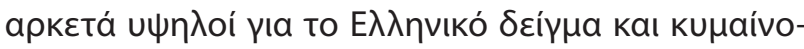

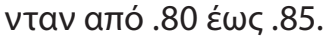

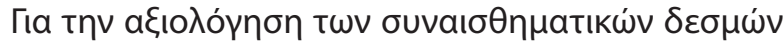

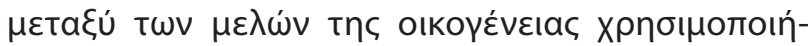

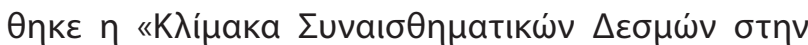

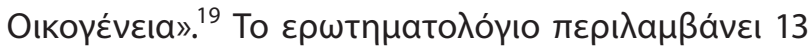

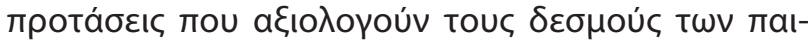

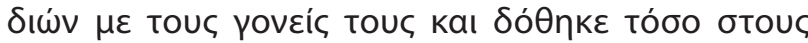

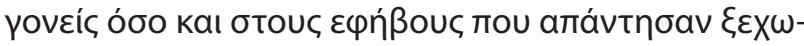

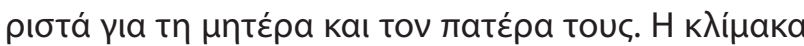

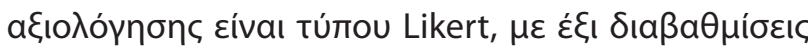

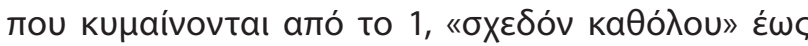

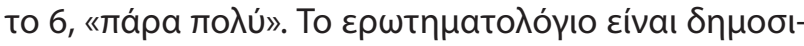

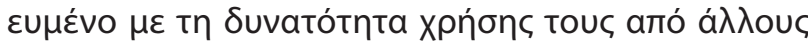

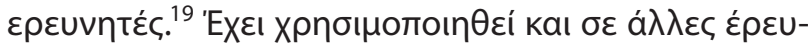

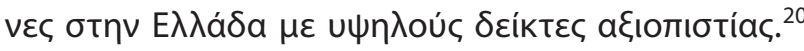

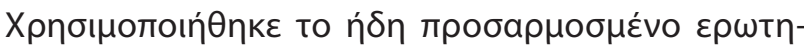

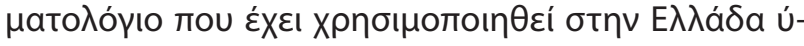

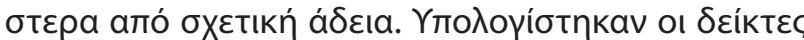

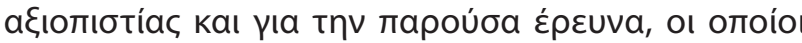

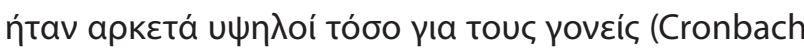

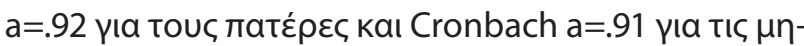

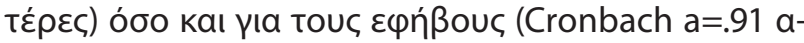

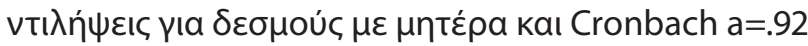

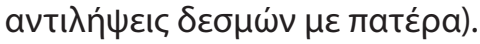

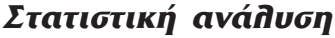

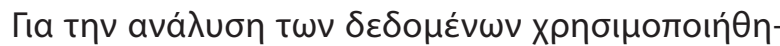

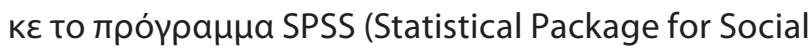

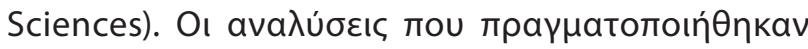

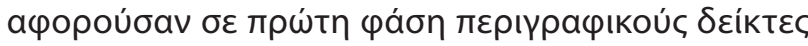

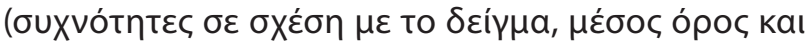

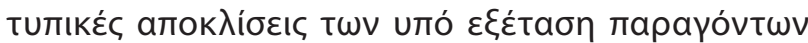

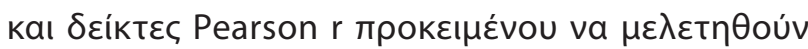

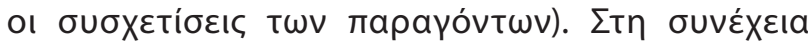

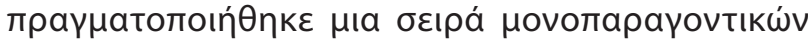

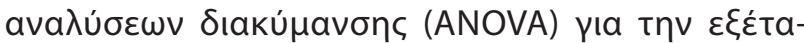

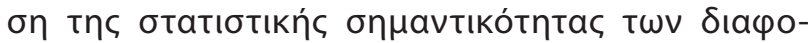

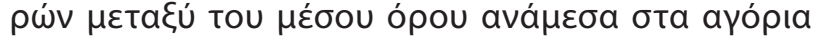

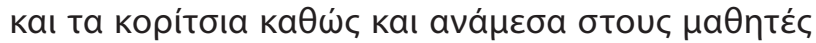

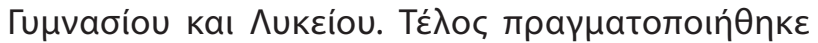

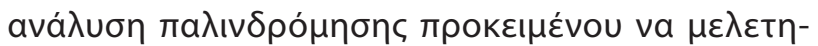
Ө

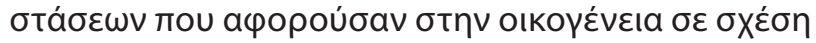

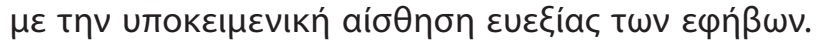

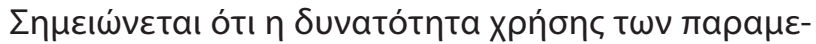

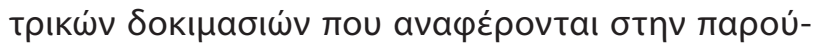

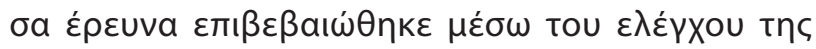

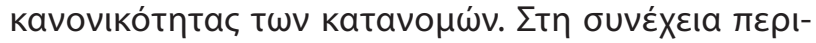

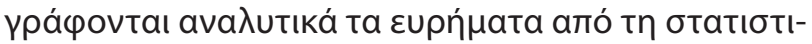

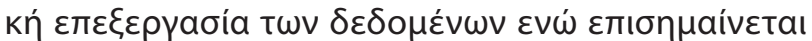

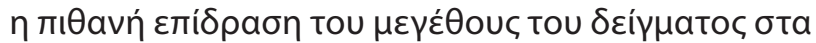

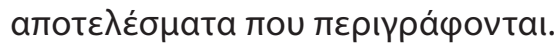

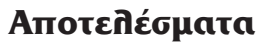

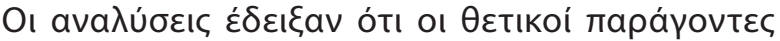

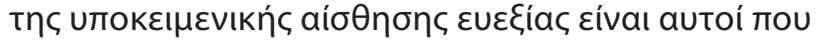

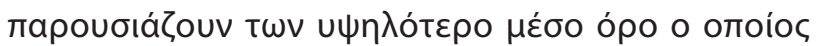

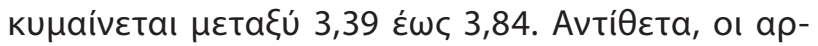

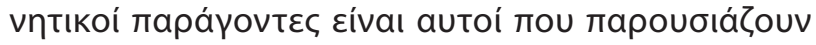

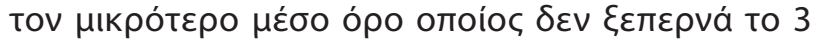
(ті́vakac 1).

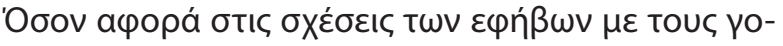

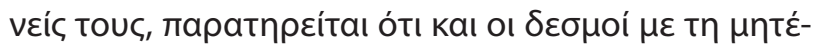

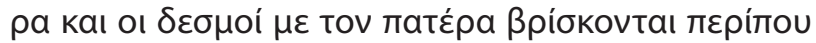

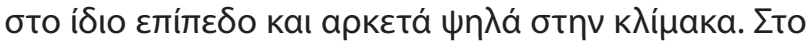

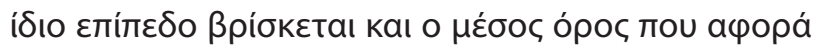

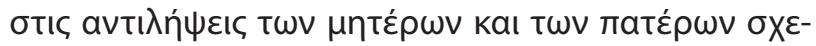

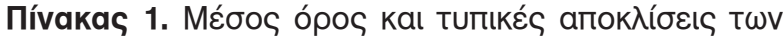

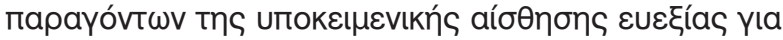

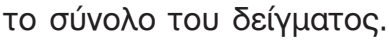

\begin{tabular}{|c|c|c|}
\hline 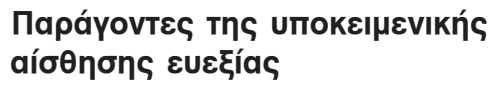 & MO & TA \\
\hline Аuтовкті́́nбп & 3,83 & 0,56 \\
\hline 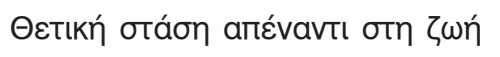 & 3,54 & 0,59 \\
\hline 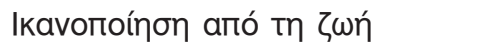 & 3,39 & 0,76 \\
\hline 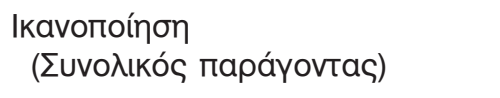 & 3,60 & 0,49 \\
\hline Проß入п́ната & 2,62 & 0,74 \\
\hline 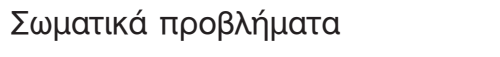 & 2,05 & 0,76 \\
\hline 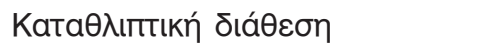 & 2,40 & 0,69 \\
\hline 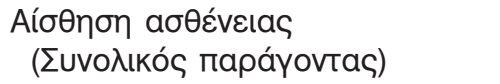 & 2,33 & 0,63 \\
\hline
\end{tabular}




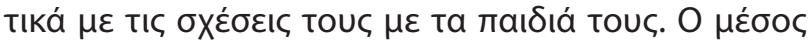

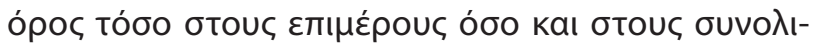

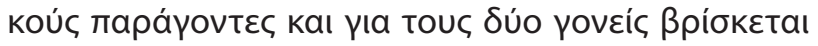

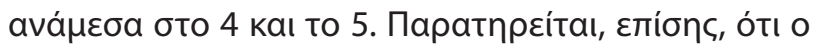

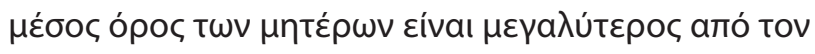

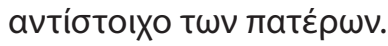

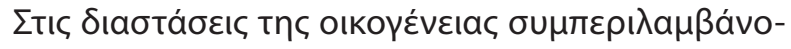

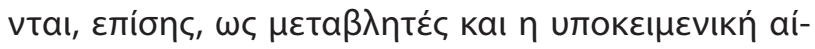

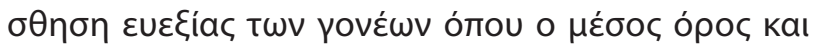

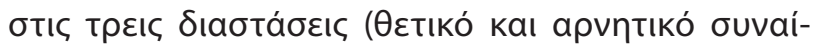

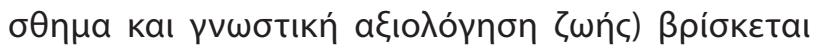

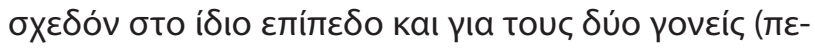

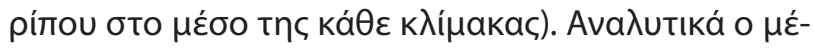

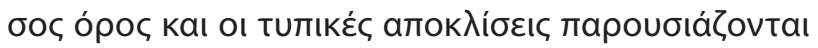
бтоv пívaкa 2.

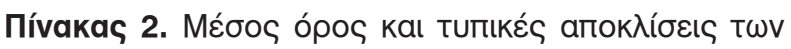

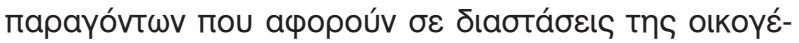

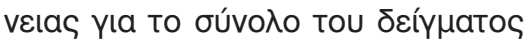

\begin{tabular}{|c|c|c|}
\hline 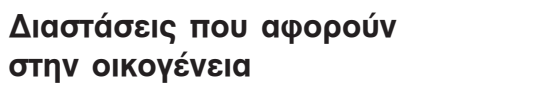 & MO & TA \\
\hline \multicolumn{3}{|l|}{ 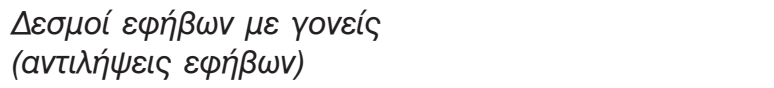 } \\
\hline 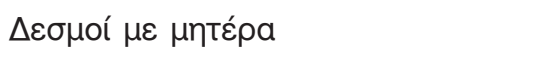 & 5,03 & 0,81 \\
\hline 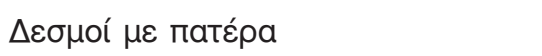 & 4,80 & 0,83 \\
\hline \multicolumn{3}{|l|}{ 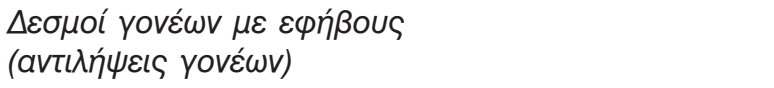 } \\
\hline 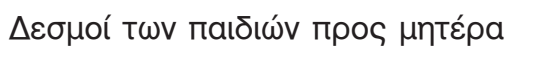 & 4,70 & 0,86 \\
\hline 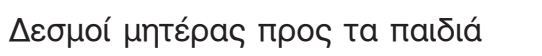 & 5,04 & 0,68 \\
\hline 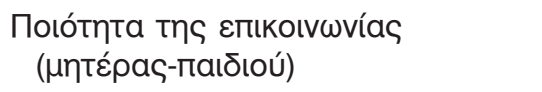 & 4,98 & 0,84 \\
\hline 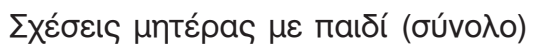 & 4,89 & 0,69 \\
\hline 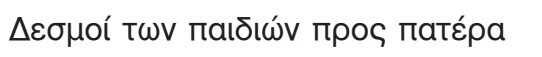 & 4,62 & 0,92 \\
\hline 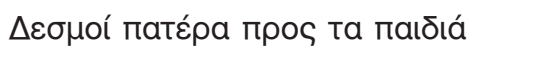 & 4,92 & 0,76 \\
\hline 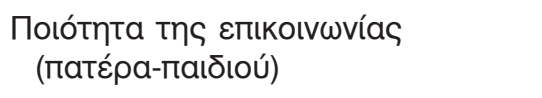 & 4,69 & 0,89 \\
\hline 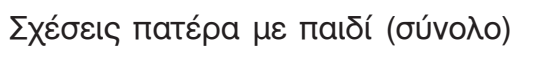 & 4,75 & 0,76 \\
\hline \multicolumn{3}{|l|}{ 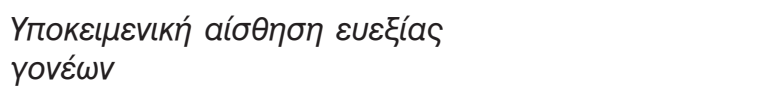 } \\
\hline 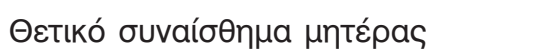 & 2,83 & 0,66 \\
\hline 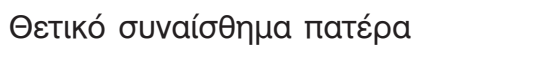 & 2,82 & 0,69 \\
\hline 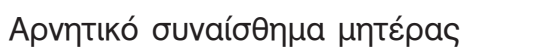 & 3,58 & 0,68 \\
\hline 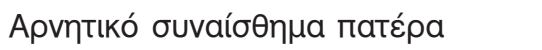 & 3,65 & 0,69 \\
\hline 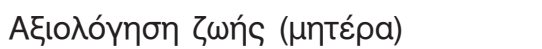 & 22,61 & 6,05 \\
\hline 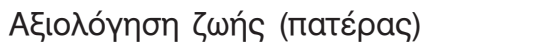 & 22,24 & 6,28 \\
\hline
\end{tabular}

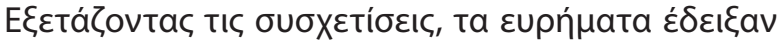

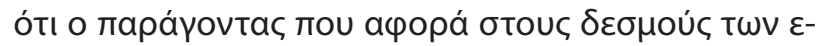

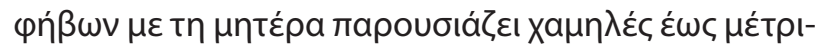

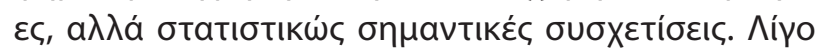

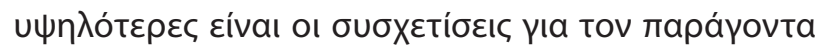

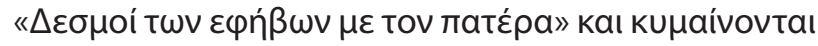

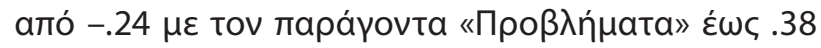

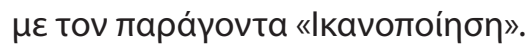

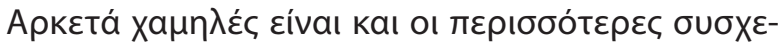

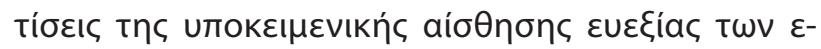

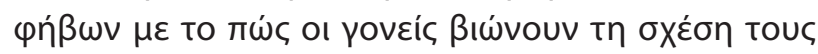

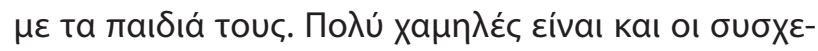

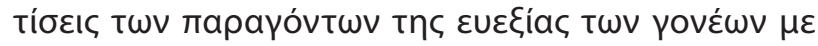

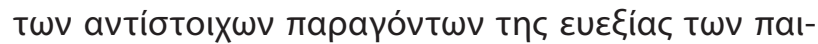

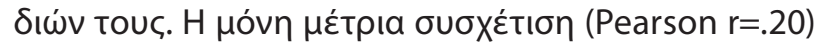

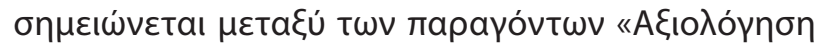

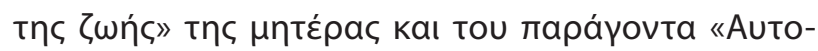

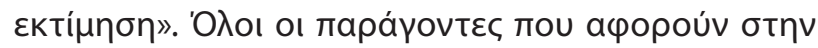

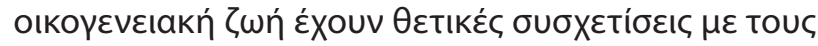

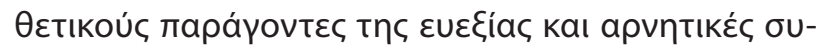

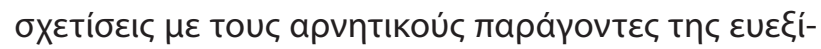

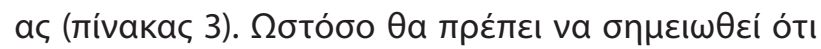

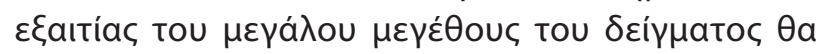

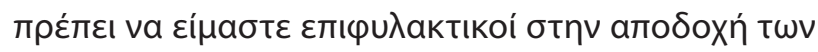

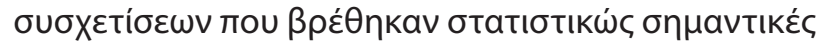

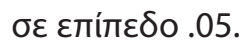

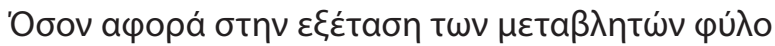

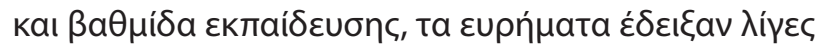

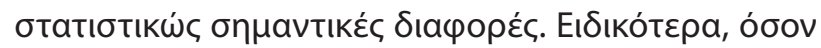

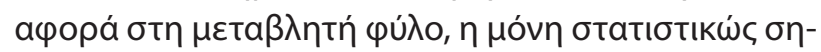

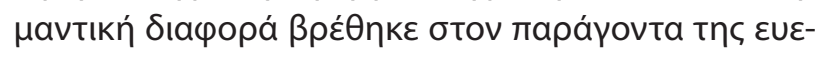

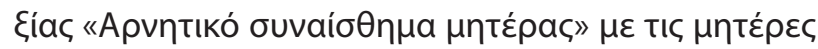

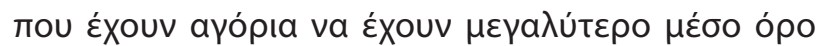

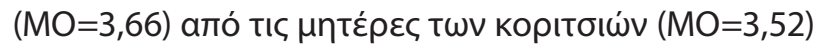

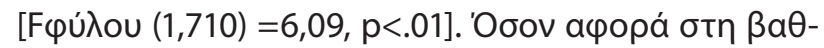

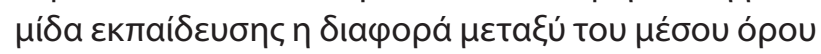

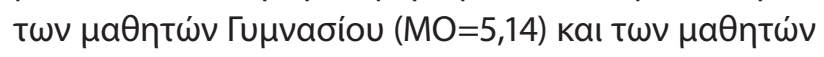

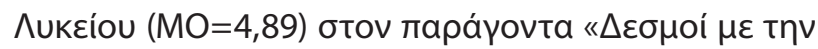

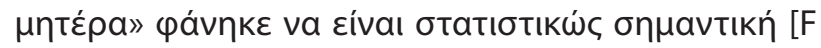

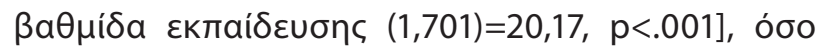

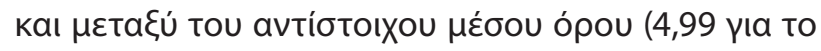

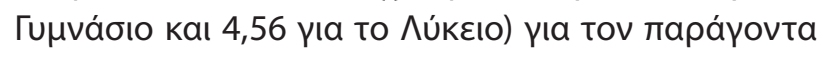

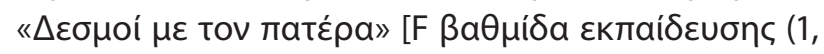
701) $=48,61, p<.001]$.

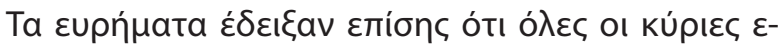

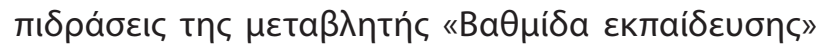

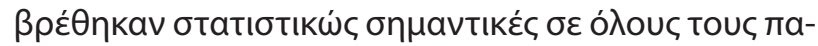




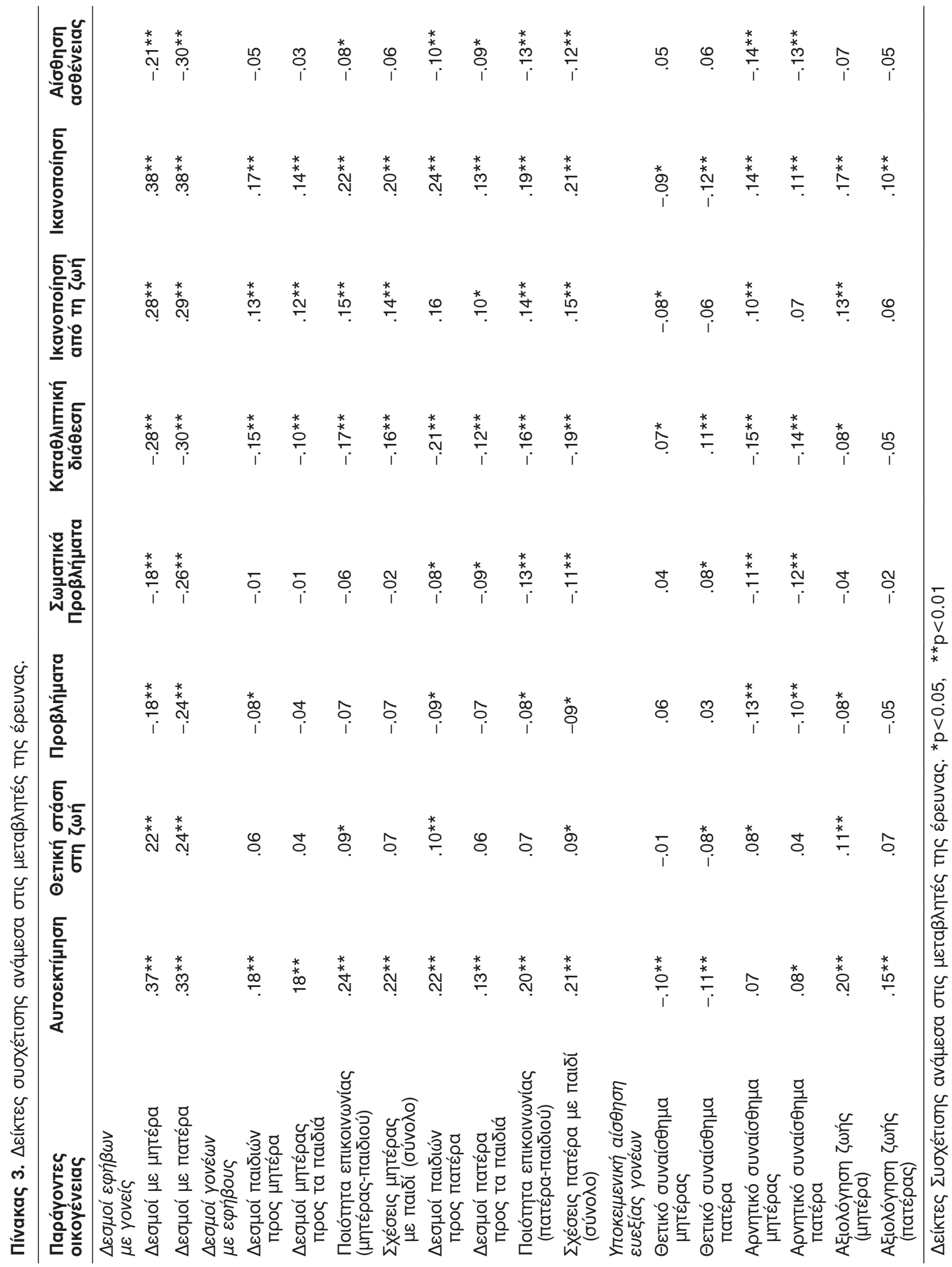




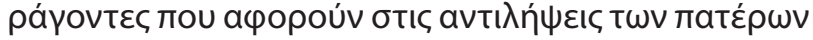

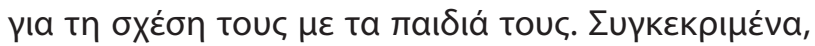

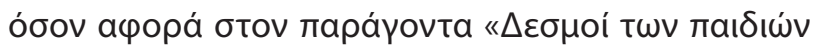

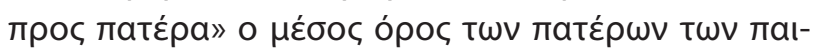

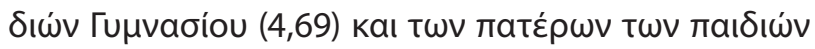

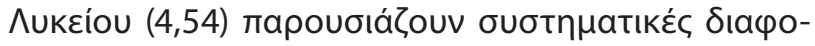

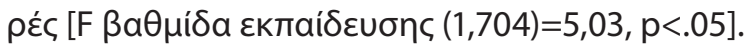

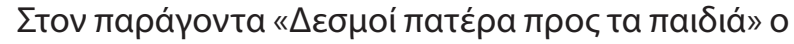

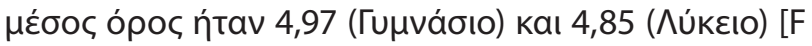

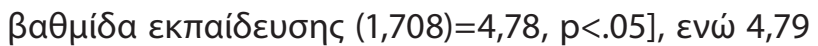

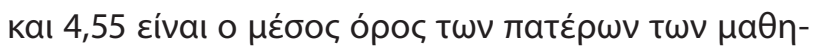

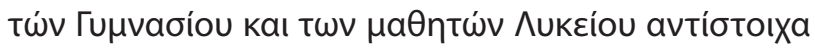

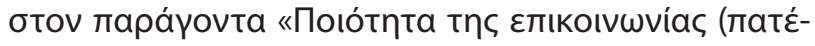

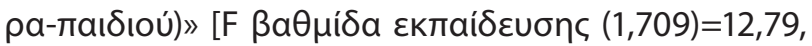

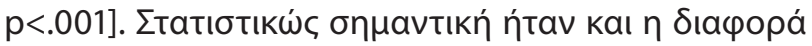

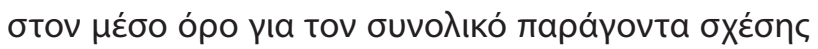

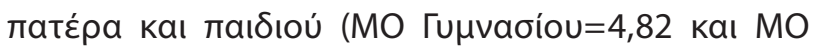

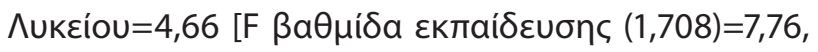
$\mathrm{p}<.01]$.

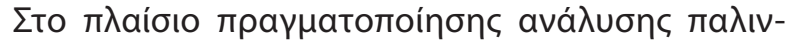

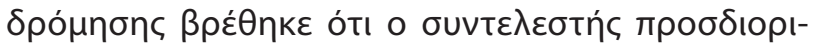

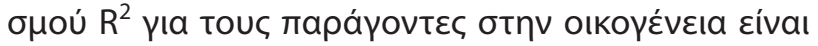

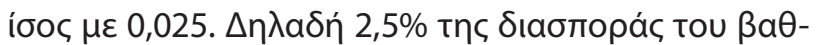

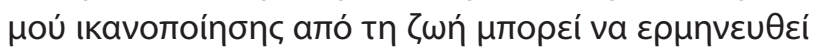

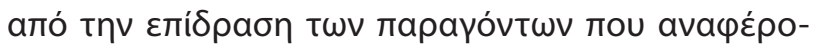

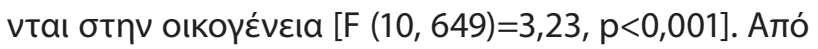

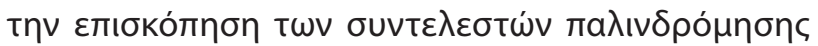

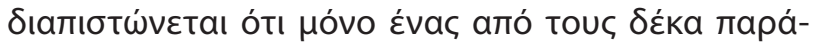

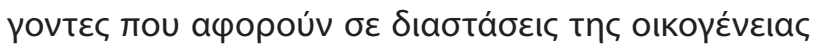

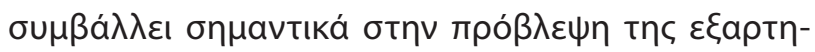

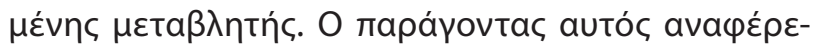

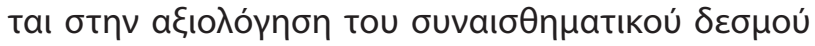

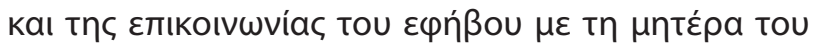
$(\beta=0,08, t=2,08, p<0,05)$. Autó on

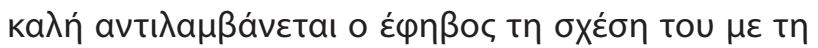

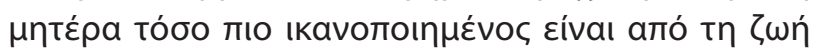

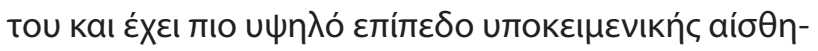

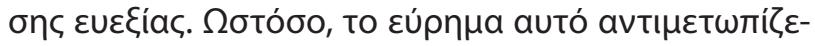

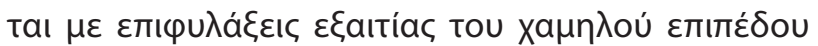

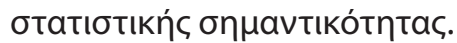

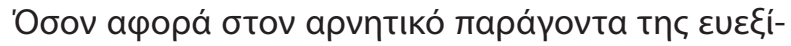

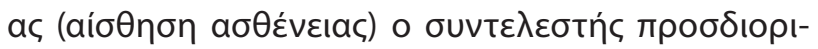

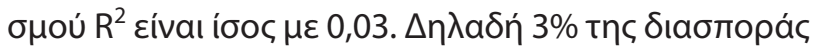

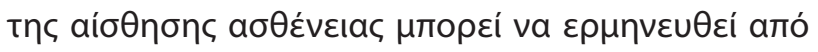

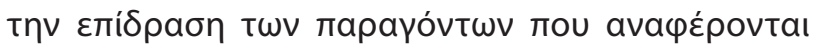

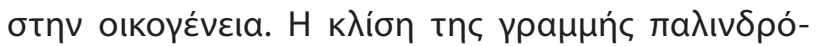

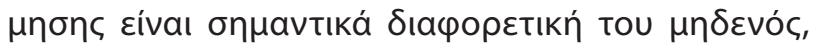

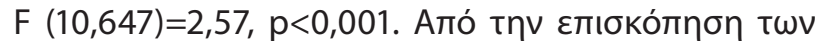

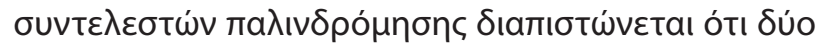

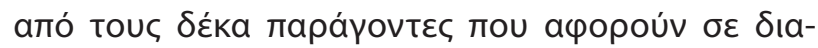

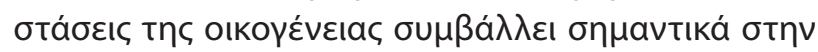

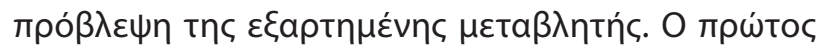

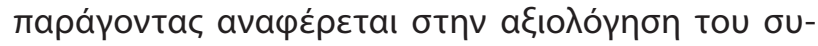

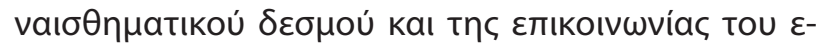

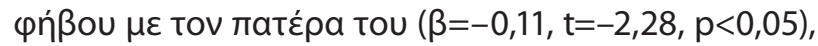

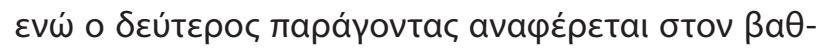

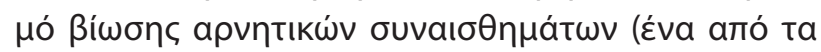

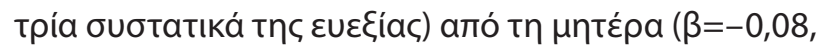

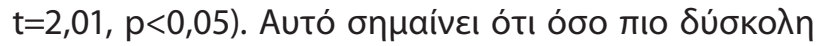

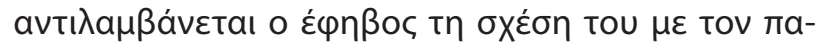

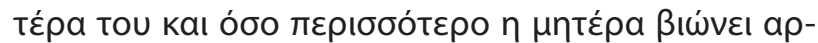

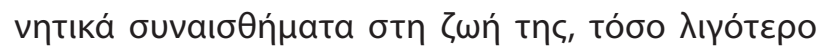

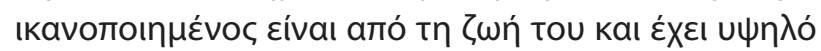

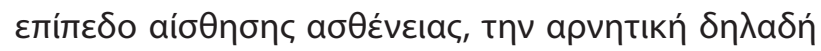

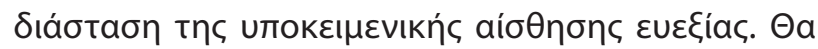

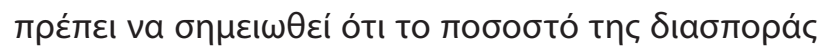

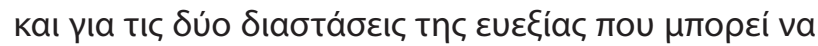

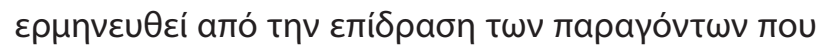

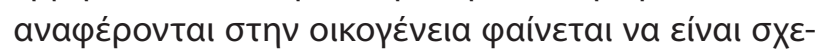

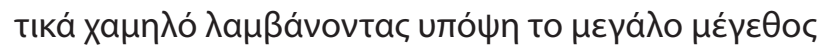

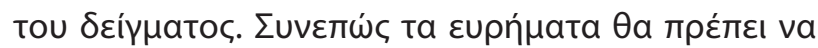

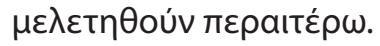

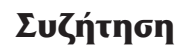

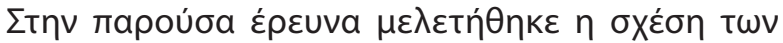

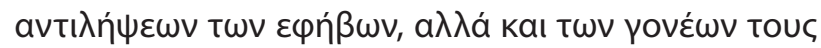

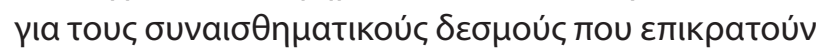

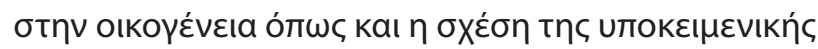

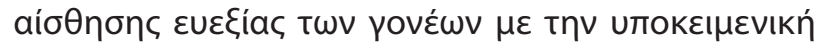

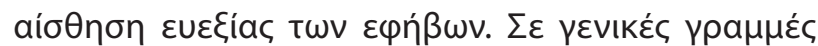

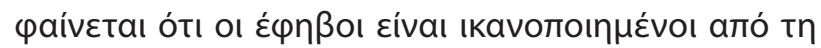

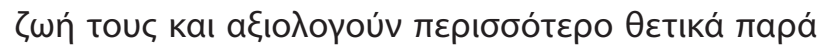

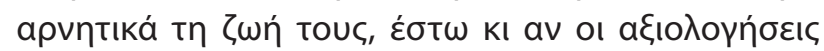

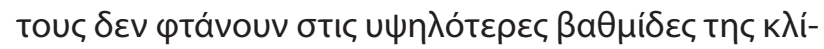

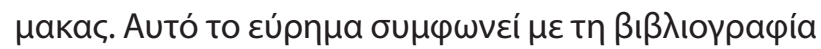

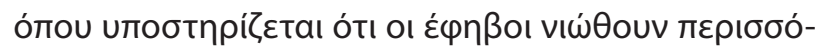

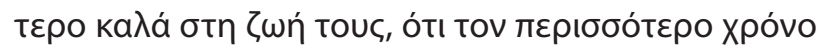

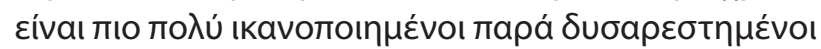

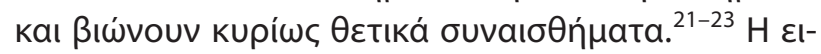

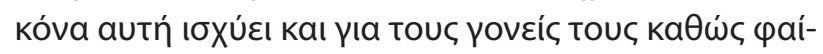

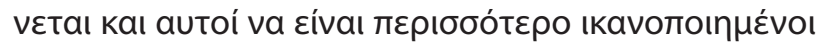

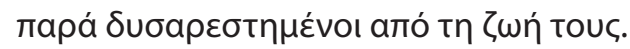

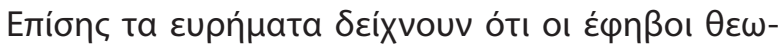

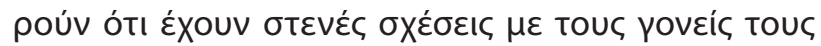




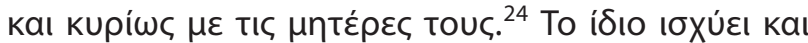

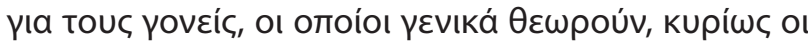

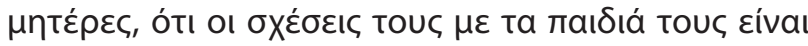

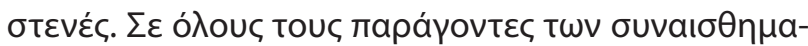

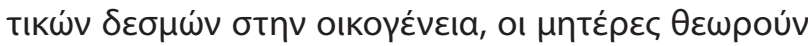

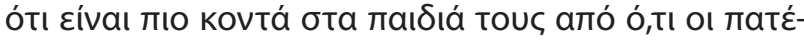

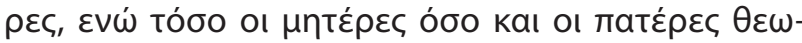

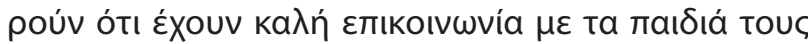

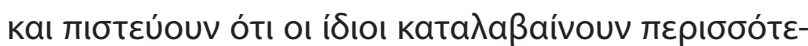

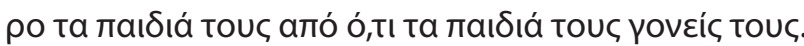

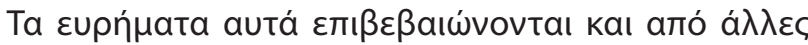

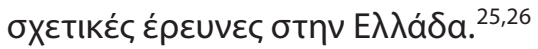

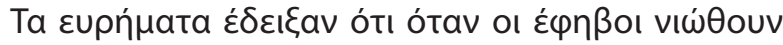

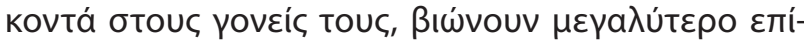

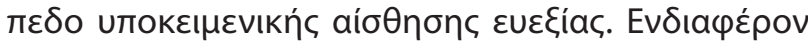

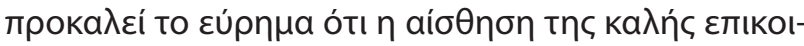

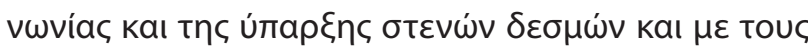

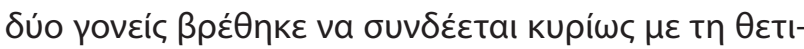

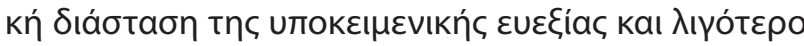

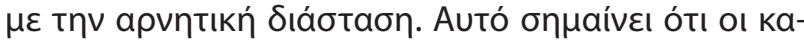

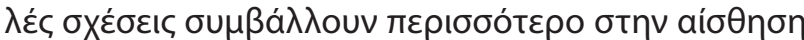

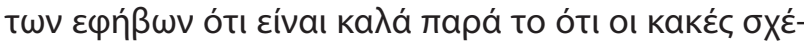

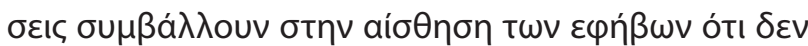

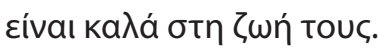

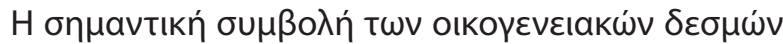

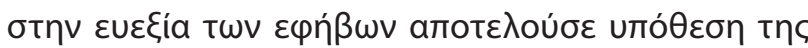

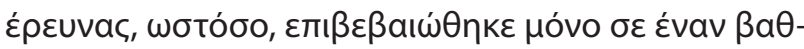
$\mu$

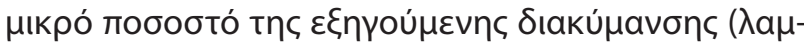

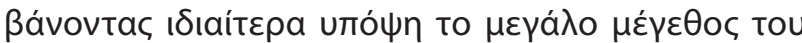

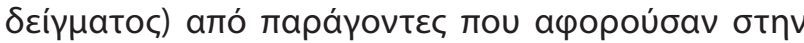

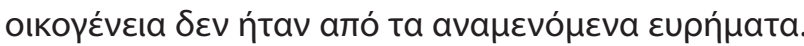

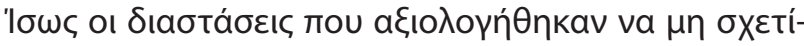

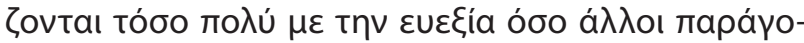

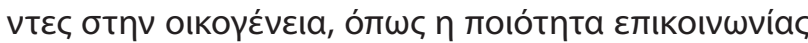

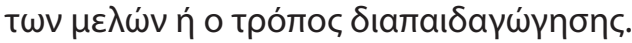

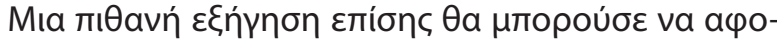

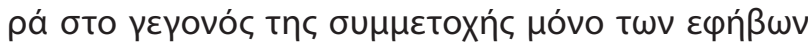

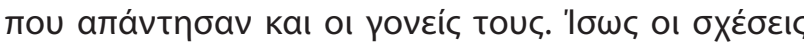

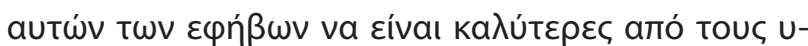

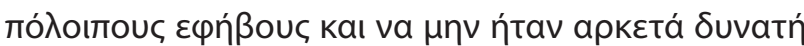

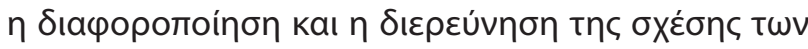
$\mu \varepsilon \tau a \beta \lambda \eta \tau \omega ́ v$.

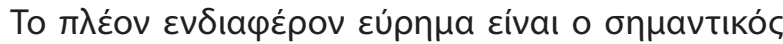

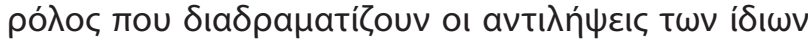

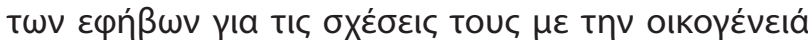

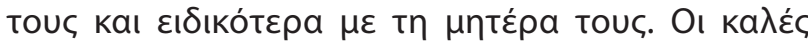

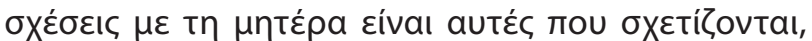

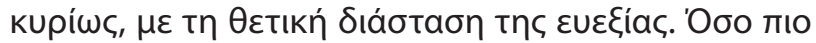

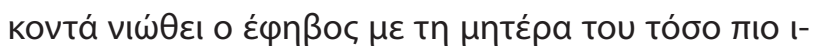

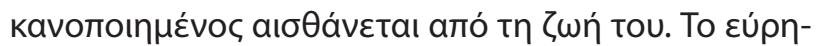

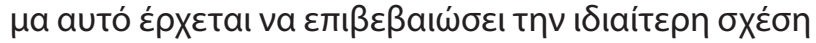

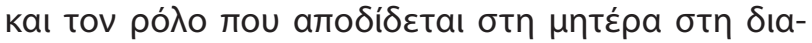

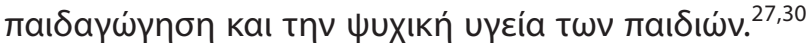

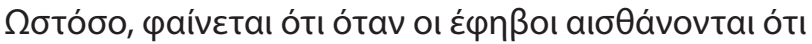

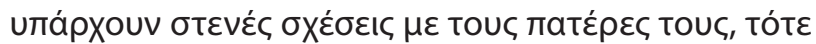

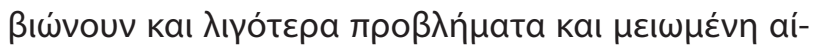
бӨnon aбӨźvelac.

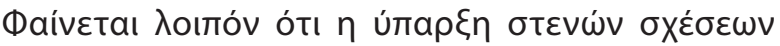

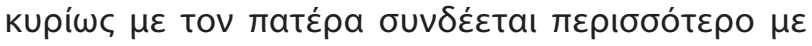

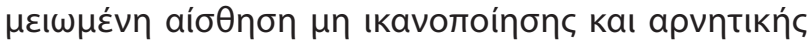

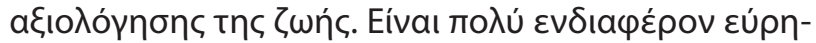

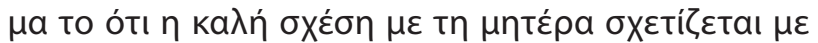

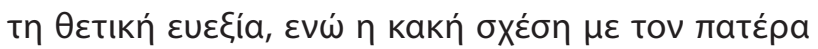

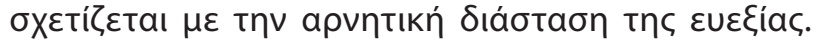

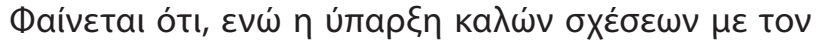

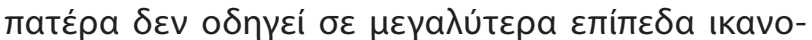

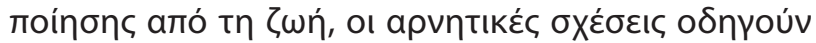

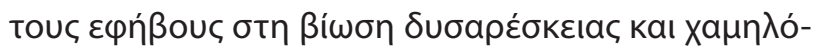

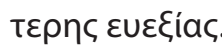

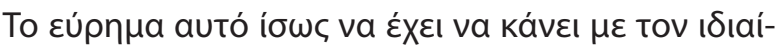

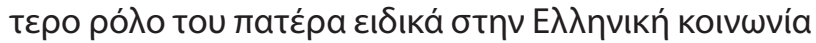

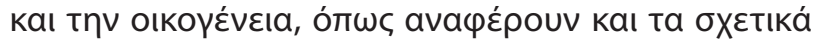

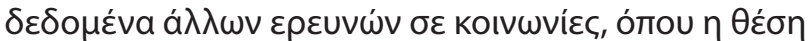

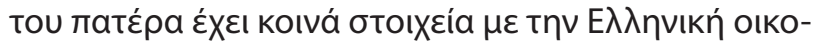

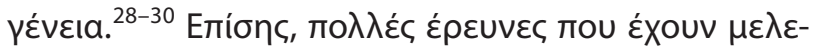

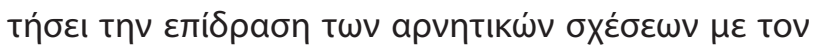

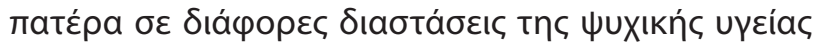

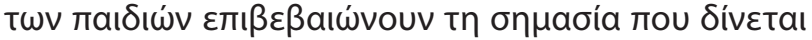

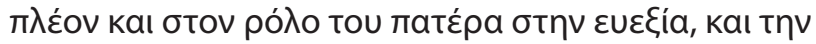

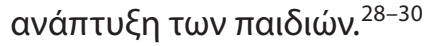

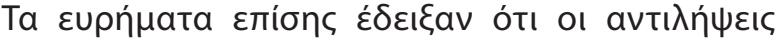

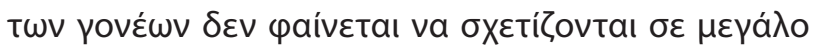

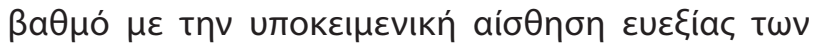

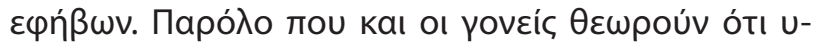

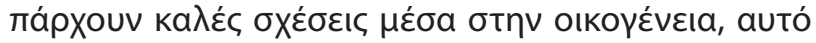

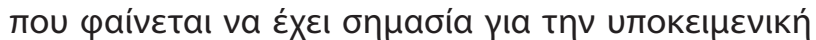

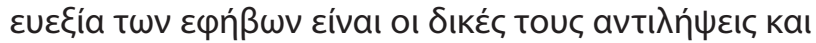

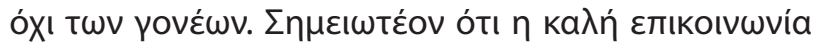

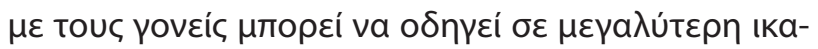

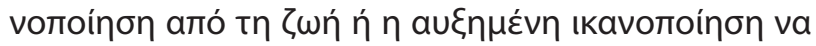

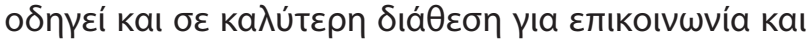

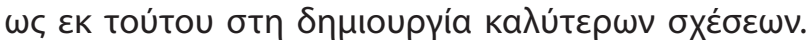

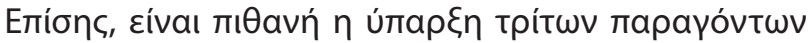




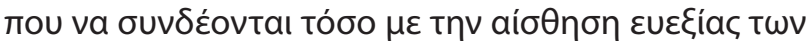

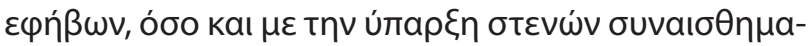

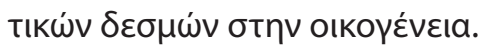

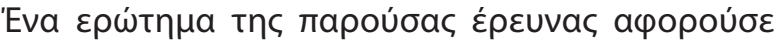

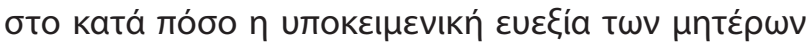

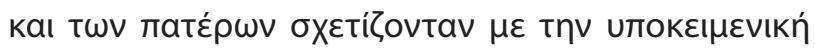

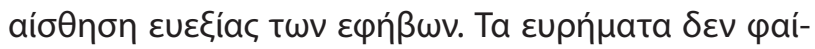

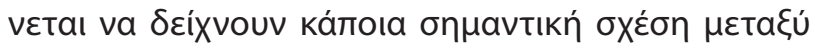

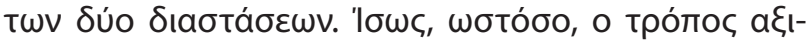

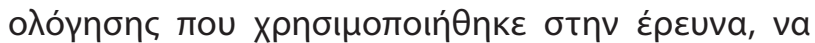

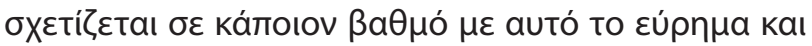

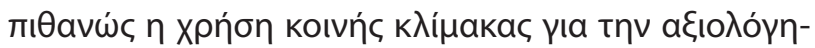

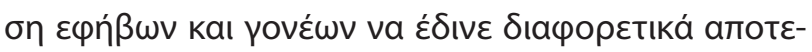

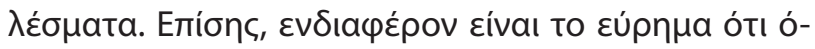

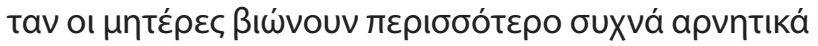

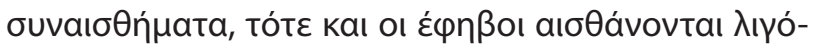

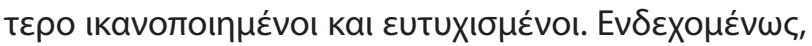

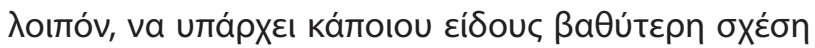

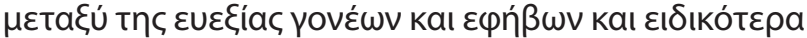

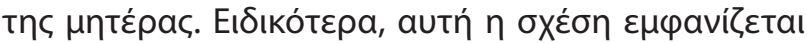

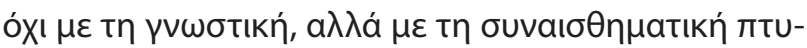

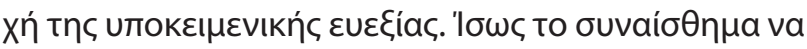

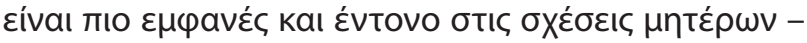

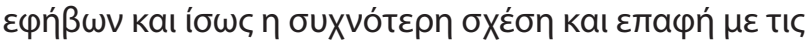

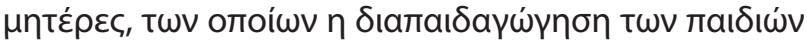

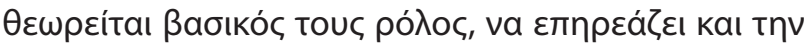

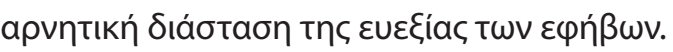

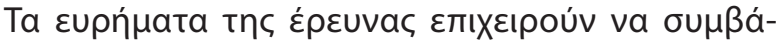

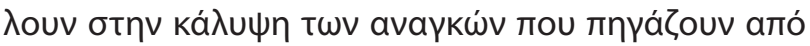

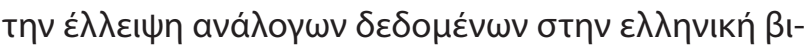

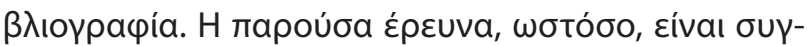

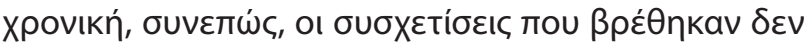

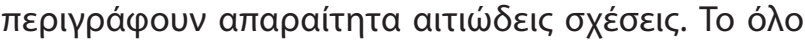

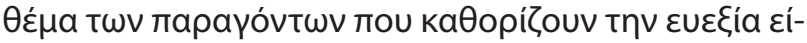

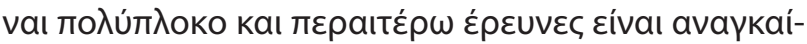

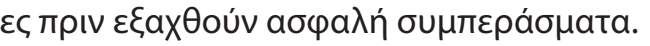

\title{
The role of the family in adolescents' subjective well-being
}

\author{
A. Lampropoulou \\ Department of Psychology, University of Athens, Athens, Greece
}

Psychiatriki 2018, 29:172-182

The goal of this research is to study the relation between adolescents' subjective well-being with: (a) their perceptions regarding their relationship with their parents, (b) the corresponding perceptions of their parents and (c) parents' subjective well-being. The participants of this study were 714 adolescents attending the two last grades of Junior High School (Gymnasium) and the two last grades of High School (Lyceum) as well as their parents from schools of the broader area of Athens randomly selected. The following questionnaires were used for data collection from the adolescents who participated in the study: (a) The assessment of subjective well-being was based on the Berne Questionnaire of Subjective Well-Being/ Youth Form that provides the following factors: Positive attitude towards life, Self-esteem, Satisfaction from life, Problems, Somatic complaints and Depressive mood. The first three factors are included in a general factor called Satisfaction which is the positive aspect of subjective well-being (according to the suggested structure of the concept in the relevant literature) while the remaining three are included in a second general factor called III-being which is the negative aspect of subjective well-being, and (b) the assessment of family relationships was based on the "Affectual solidarity in the family scale" (which was also administered to parents). In addition, parents completed the questionnaires "Satisfaction from Life scale" and the "Assessment of the emotional satisfaction from life-Keyes scale" for assessing their level of subjective well-being. Finally, information on demographics was also collected. Statistical analysis of the data included descriptive and deductive methods in order to examine the relationship and the interaction of the variables under research. Findings revealed several interesting relationships. In particular 
the results showed that the better the relationships are perceived by the adolescents the more satisfied they feel from their lives. In particular, the good relationships with the mother strengthen the positive dimension of subjective well-being while the good relationships with the father decrease the negative dimension of subjective well-being. Findings also suggest no significant relation among parents' perceptions and their subjective well-being with the well-being of the adolescents. The results of the research provide the guidelines for further research on the role of the family in adolescents' subjective well-being while they also allow for a better understanding of the way adolescents' subjective well-being is related to various variables in order to further enhance it through appropriate interventions.

Key words: Adolescence, subjective well-being, family, satisfaction from life.

\section{BıBAtoypacpía}

1. Brotherson SE, Yamamoto T, \& Acock AC. Connection and communication in father-child relationships and adolescent child well-being. Fathering 2003, 1:191-214, doi: 10.3149/ fth.0103.191

2. Diener E. Subjective well-being: The science of happiness and a proposal for a national index. Am Psychol 2000, 55:34-43, doi: 10.1037/0003-066X.55.1.34

3. Karademas EC. Self-efficacy, social support and well-being: The mediating role of optimism. Pers Individ Dif 2006, 40:1281-1290, doi: 10.1016/j.paid.2005.10.019

4. Karademas EC. Subjective well-being, demographic and intrapersonal correlates. $\Psi_{u}$ Xo ${ }^{\prime}$ oyía in press

5. Behnke AO, MacDermid SM. Family well-being. A Sloan work and family encyclopedia entry 2004, Available from: http:// www. bc.edu/bc_org/

6. Hann CM. The relationship between certain family variables and the psychological well-being of black adolescents, 2005, Doctoral dissertation, University of the Free State, Bloemfontein Available from: http://www.scholar.ufs.ac.za

7. Hetherington EM. Social support and the adjustment of children in divorced and remarried families, Childhood 2003, 10(2): 217-36, doi: 10.1177/0907568203010002007

8. Amato PR, \& Afifi TD. Feeling caught between parents: adult children's relations with parents and subjective well-being, J Marriage Fam 2006, 68:222-235, doi: 10.1111/j.17413737.2006.00243

9. Rask K, Astedt-Kurk P, Paavilainen E, \& Laippala P. Adolescent subjective well-being and family dynamics. Scand J Caring Sci 2003, 17:129-138, doi: 10.1037/0033-2909.132.2.249

10. Ryff CD, Singer BH, Wing E, \& Love GD. Elective affinities and uninvited agonies: mapping emotion with significant others onto health. In: Ryff CD. \& Singer BH (eds) Emotion, Social Relationships, and Health, New York, NY: Oxford University Press, 2001:133-175, doi: 10.1093/acprof:oso/9780 195145410.003.0005

11. Johnson LD, O'Malley PM, Bachman JG. National survey results on drug use from the monitoring the future study, 1975-2000: Vol. 1. Secondary school students (NIH Publication No. 2000). Rockville, MD: National Institute on Drug Abuse. 2001 Available from: http://www.monitoringthefuture.org/pubs/monographs/ vol1_2002.pdf
12. Ryan RM, Deci EL. On happiness and human potentials: A review of research on hedonic and eudaimonic well-being. Annu Rev Psychol 2001, 52:141-166, doi: 10.1146/annurev. psych.52.1.141

13. Flouri E. Subjective well-being in midlife: the role of involvement of and closeness to parents in childhood. $J$ Happiness Stud 2004, 5: 335-358, doi: 10.1023/b:johs.0000048461.21694.92

14. Joronen K, Astedt-Kurki P. Familial contribution to adolescent subjective well-being. Int J Nurs Stud 2005, 11:125-133, doi: 10.1111/j.1440-172X.2005.00509.x

15. Grob A, Luthi R, Kaiser F, Flammer A, Mackinnon A, Wearing A. Berner fragebogen zum wohlbefinden jugendlicher (BSW-Youth Form), Diagnostica 1991, 37:66-75 Available from: http://edoc. unibas.ch/dok/A5255072

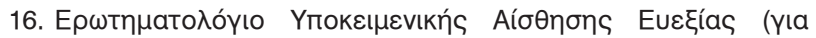

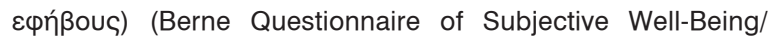

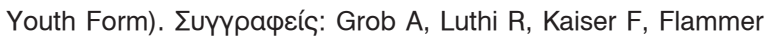

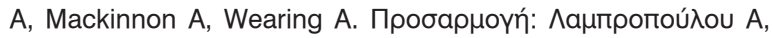

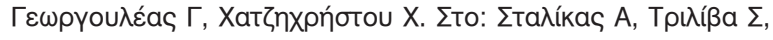

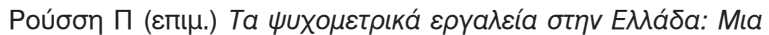

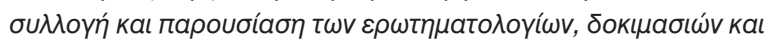

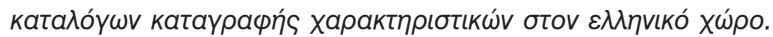
AӨńva, Пєסío. 2012:713

17. Diener E, Emmons RA, Larsen RJ, Griffin S. The Satisfaction with Life Scale. J Pers Assess 1985, 49:71-75, doi: 10.1037/10403590.5.2.164

18. Keyes CLM, Social well-being. Soc Psychol Q 1998, 61:121-140, doi: $10.2307 / 2787065$

19. Gronvold RL. Measuring affectual solidarity. In: Mangen D, Vern J. Bengtson L, Landry P (eds) The measurement of intergenerational relations. Beverly Hills, CA: Sage, 1988:74-97 Available from: http://psycnet.apa.org/record/1988-97094-004

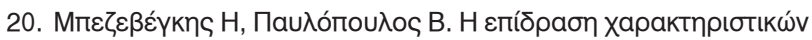

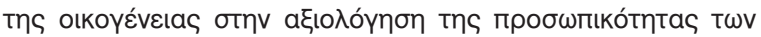

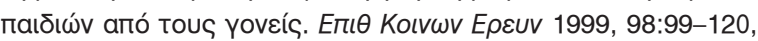
doi: $10.12681 / \mathrm{grsr} .744$

21. Gilman R, Huebner S. A review of life satisfaction research with children and adolescents. Sch Psychol Q 2003, 18:192-205, doi: 10.1521/scpq.18.2.192.21858

22. Karademas EC. Positive and negative aspects of well-being: Common and specific predictors. Pers Individ Dif 2007, 43:277287, doi: 10.1016/j.paid.2006.11.031 


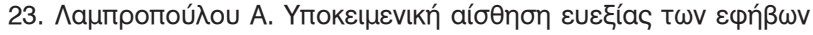

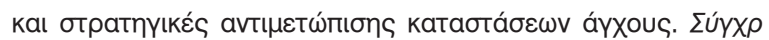

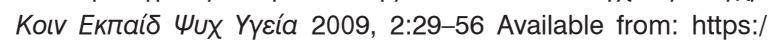
aigaion.org/skepsy

24. Lampropoulou A, Georgouleas G, Hatzichristou C. Subjective well-being and coping strategies of Greek adolescents. Poster presented at IXth Conference of European association for research on Adolescence, Porto 2004

25. Georgouleas G, Besevegis E. Adolescents' views of their rela-

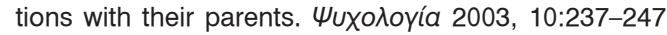

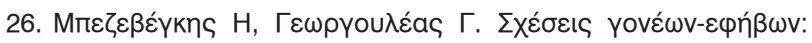

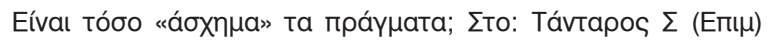

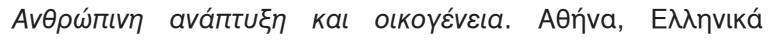
Гра́ $\mu$ ata, 2004:103-12

27. Li-xing Z, Guang-fang T, The influential factors on quality of life in children. Chin J Clin Psychol 2001, 9:105-107 Available from: https://caod.oriprobe.com/articles/3262155

28. Flouri E, Buchanan A. Childhood predictors of labor force participation in adult life. JFEI 2002, 23:101-120, doi: 10.1023/ A: 1015776832505
29. Shek DTL. The relation of parental qualities to psychological well-being, school adjustment and problem behavior in Chinese adolescents with economic disadvantage. Am J Fam Ther 2002, 30:215-230, doi: 10.1080/019261802753577548

30. Maccoby EE. Parenting and its effects on children: On reading and misreading behavior genetics. Annu Rev Psychol 2000, 51:1-27, doi: 10.1146/annurev.psych.51.1.1

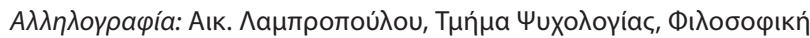

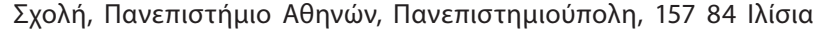

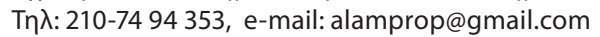




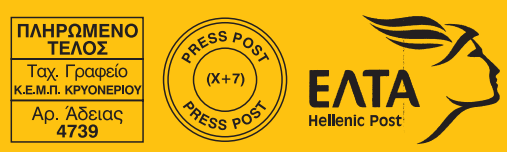

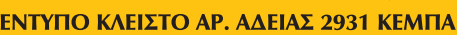

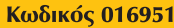

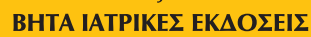

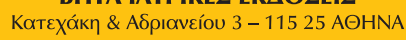

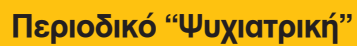

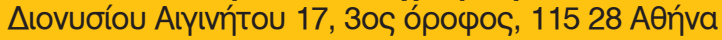

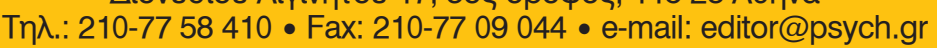

The Journal "Psychiatriki"

17, Dionisiou Eginitou str., 3rd floor, 11528 Athens, Greece

Tel.: (+30) 210-77 58410 • Fax: (+30) 210-210-77 09044 • e-mail: editor@psych.gr

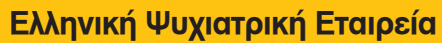

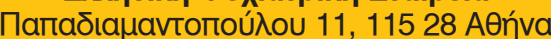

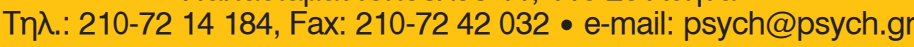

Hellenic Psychiatric Association

11, Papadiamantopoulou str., 11528 Athens, Greece

Tel.: (+30) 210-72 14 184, Fax: (+30) 210-72 42032 •e-mail: psych@psych.gr 